\title{
Direct Access to Highly Enantioenriched $\alpha$-Branched Acrylonitriles through a one-pot Sequential Asymmetric Michael addition/retro-Dieckmann/retro-Michael Fragmentation Cascade
}

Nicolas Duchemin, ${ }^{\mathrm{a}}$ Martin Cattoen, ${ }^{\mathrm{a}}$ Oscar Gayraud, ${ }^{\mathrm{a}}$ Silvia Anselmi, ${ }^{\text {a }}$ Bilal Sidiq, ${ }^{\mathrm{a}}$ Roberto Buccafusca, ${ }^{a}$ Marc Daumas, ${ }^{b}$ Vincent Ferey, ${ }^{c}$ Michael Smietana, ${ }^{d}$ Stellios Arseniyadis. ${ }^{a}, *$

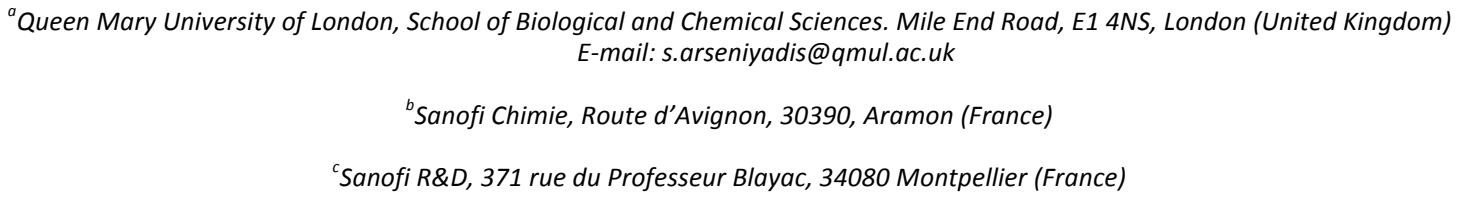

${ }^{b}$ Sanofi Chimie, Route d'Avignon, 30390, Aramon (France)

${ }^{c}$ Sanofi R\&D, 371 rue du Professeur Blayac, 34080 Montpellier (France)

\section{Supporting Information}


Table of content

Material and methods

List of compounds

Experimental and Spectral data

I. General Procedures: Substrate synthesis

II. General Procedures: Alkenyl nitriles synthesis

- Optimization

- Mechanistic hypothesis

- Sc-Catalyzed sequence

- Cu-Catalyzed sequence 


\section{Materials and Methods}

The reactions were run under argon atmosphere in oven-dried glassware unless otherwise specified. All commercially available compounds were purchased from Sigma Aldrich, Fluorochem, or Alfa Aesar, and used as received. Reactions ran at temperatures were conducted in sealed microwave tubes in an acetone bath which temperature was controlled by a cryostat. Analytical thin layer chromatography (TLC) was performed on silica gel plates (Merck 60F254) visualized either with a UV lamp (254 $\mathrm{nm}$ ) or by using solutions of $\mathrm{KMnO}_{4} / \mathrm{K}_{2} \mathrm{CO}_{3} / \mathrm{ACOH}$ in water followed by heating using a heating gun. Flash chromatographies were performed on silica gel (60-230 mesh). Organic extracts were dried over anhydrous $\mathrm{MgSO}_{4} .{ }^{1} \mathrm{H}$ NMR spectra were recorded on a Bruker AVANCE 400 at 400 $\mathrm{MHz}$ in $\mathrm{CDCl}_{3}$ and the observed signals are reported as follows: chemical shift in parts per million from tetramethylsilane with the solvent as an internal indicator $\left(\mathrm{CDCl}_{3} \delta 7.26 \mathrm{ppm}\right)$, multiplicity $(\mathrm{s}=$ singlet, $\mathrm{d}=$ doublet, $\mathrm{t}=$ triplet, $\mathrm{q}=$ quartet, $\mathrm{p}=$ pentet, $\mathrm{m}=$ multiplet or

overlap of non equivalent resonances, $\mathrm{br}=$ broad), integration. ${ }^{13} \mathrm{C} N \mathrm{NMR}$ spectra were recorded at $101 \mathrm{MHz}$ in $\mathrm{CDCl}_{3}$ and the observed signals were reported as follows: chemical shift in parts per million from tetramethylsilane with the solvent as an internal indicator $\left(\mathrm{CDCl}_{3} \delta 77.0 \mathrm{ppm}\right)$. Coupling constants, $J$, are reported in Hertz $(\mathrm{Hz}) .{ }^{19} \mathrm{~F} \mathrm{NMR}$ spectra were recorded on a AVANCE 400 (Bruker) at $377 \mathrm{MHz}$ in $\mathrm{CDCl}_{3}$ and the observed signals are reported as follows: chemical shift in parts per million (calibrated from the ${ }^{1} \mathrm{H}$ NMR spectra), multiplicity $(\mathrm{s}=$ singlet, $\mathrm{d}=$ doublet, $\mathrm{t}=$ triplet, $\mathrm{q}=$ quartet, $\mathrm{p}=$ pentet, $\mathrm{m}=$ multiplet or overlap of non equivalent resonances), integration. All NMR spectra were obtained at room temperature. Mass spectra (MS) were recorded using a HDMS Synapt G2Si Q-TOF Mass spectrometer (Waters). High Performance Liquid Chromatography (HPLC) was carried out on an Agilent system using chiral columns (Daicel). IR spectra were recorded on a Cary 630 FTIR (Agilent Technologies). Specific rotations were recorded on a AA-100 Polarimeter (Optical Activity Ltd). 


\section{List of compounds}

$\mathbb{L}_{N_{1 a}^{N}}^{O}$<smiles>C/C=C/C(=O)c1nccn1C</smiles><smiles>C/C=C/C(=O)c1nccn1C</smiles>

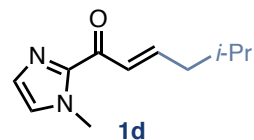<smiles>Cn1ccnc1C(=O)/C=C/CC1CCCCC1</smiles><smiles>Cn1ccnc1C(=O)/C=C/c1ccccc1</smiles><smiles>C=CC=CC(=O)c1nccn1C</smiles><smiles>C=CC=CC(=O)c1nccn1C</smiles><smiles>COC/C=C/C(=O)c1nccn1C</smiles><smiles>Cn1ccnc1C=CCOCCO</smiles><smiles>Cn1ccnc1C(=O)/C=C/C=C/c1cc2ccccc2n1C</smiles>

$1 \mathrm{k}$<smiles>O=C(/C=C/C(F)(F)F)c1nccn1I</smiles><smiles></smiles>

$1 \mathrm{~m}$<smiles>Cn1ccnc1C(=O)/C=C/c1ccccc1</smiles>

$1 r$<smiles>Cn1ccnc1C(=O)/C=C/c1cccc(Br)c1</smiles><smiles>CC(C)/C=C/C(=O)c1nccn1C</smiles>

$1 n$<smiles>Cn1ccnc1C(=O)/C=C/C1CCCCC1</smiles>

10<smiles></smiles>

$1 p$<smiles>Cn1ccnc1C=CC1CCOCC1</smiles>

$1 q$<smiles>COc1ccc(/C=C/c2c(C(=O)/C=C/c3ccc(OC(=O)C(=O)c4nccn4C)cc3)ncn2C)cc1</smiles><smiles>Cn1ccnc1C(=O)/C=C/c1ccco1</smiles><smiles>Cn1ccnc1C(=O)/C=C/c1cccs1</smiles><smiles>CC=CC=CC(=O)c1nccn1C</smiles>
1af 


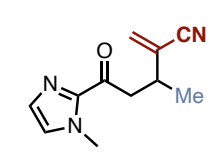

$3 a$

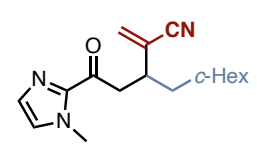

$3 e$

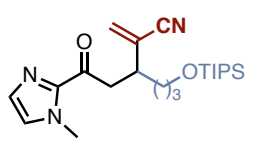

$3 \mathbf{i}$

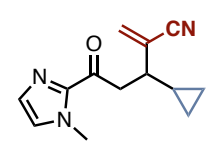

$3 \mathrm{~m}$

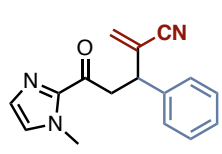

$3 r$
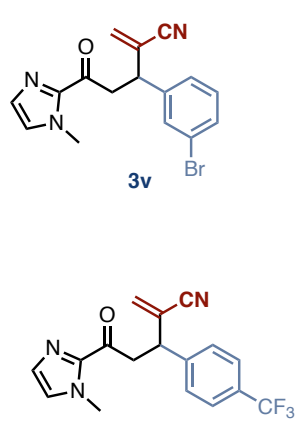

$3 z$

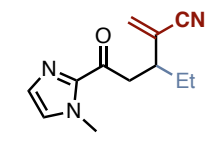

3b

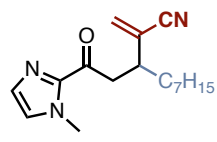

3c

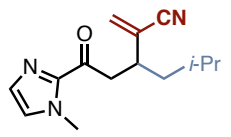

3d

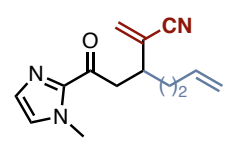

$3 g$

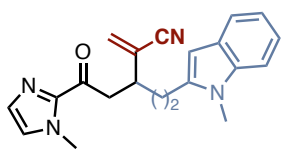

$3 k$

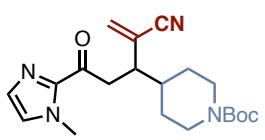

$3 p$

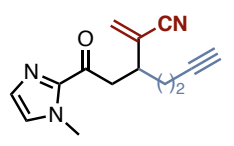

3h

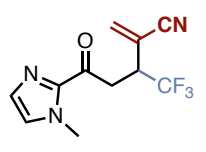

3।

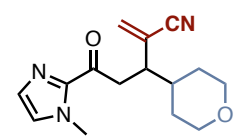

$3 q$
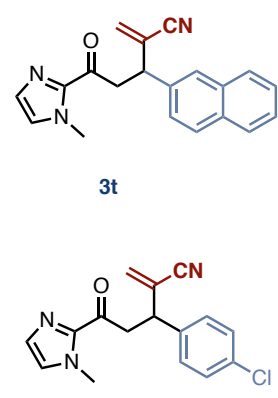

$3 x$

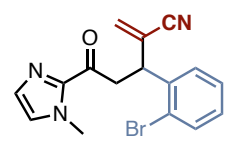

$3 u$

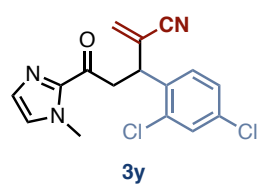

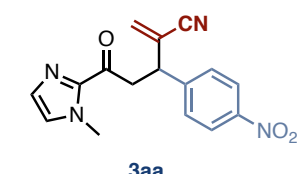
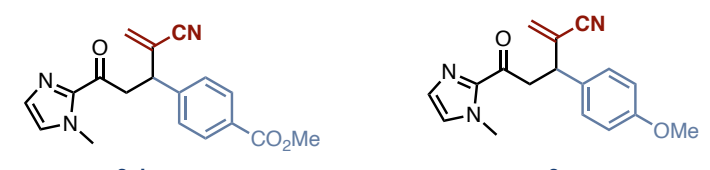<smiles>C=C(C)C(CC(=O)c1nccn1C)c1ccco1</smiles><smiles>C=C(C(=O)c1nccn1C)C(CC(=O)c1cccs1)c1cccnc1</smiles><smiles>C=C(N)C(C=CC)CC(=O)c1nccn1C</smiles>

11 $\mathrm{y}_{\mathrm{Me}}^{\mathrm{CN}}$
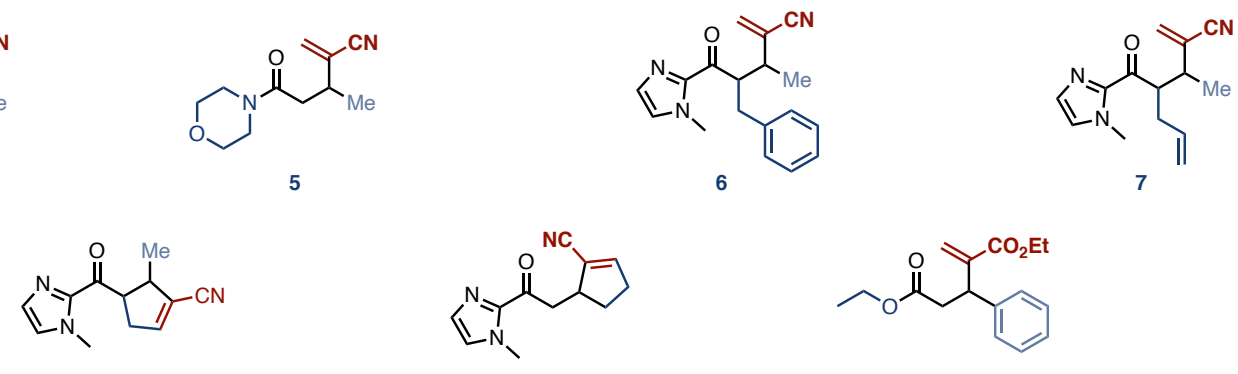

$\overbrace{1}^{\mathrm{CO}_{2} \mathrm{Et}}$

10 


\section{General Procedures: Substrate synthesis}

\section{General procedure A for the aldolisation with LDA}

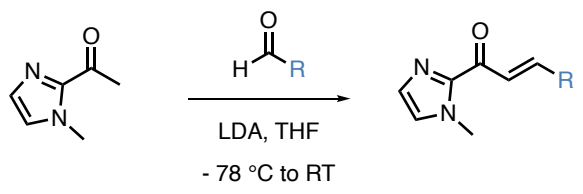

To a solution of diisopropylamine (1.3 equiv.) in THF ( $3 \mathrm{~mL} / \mathrm{mmol}$ ) was added $n$-BuLi $(2.5 \mathrm{M}$ in hexane, 1.2 equiv.) dropwise at $-78^{\circ} \mathrm{C}$. After stirring for $30 \mathrm{~min}$ at $\mathrm{rt}$, the reaction mixture was cooled to $-78^{\circ} \mathrm{C}$ again and a solution of methylketone $\mathbf{i}$ (1.0 equiv.) in THF ( $3 \mathrm{~mL} / \mathrm{mmol}$ ) was dropwise added. The solution was stirred at $\mathrm{rt}$ for $60 \mathrm{~min}$. After cooling to $-78{ }^{\circ} \mathrm{C}$, a solution of the desired aldehyde (1.5 equiv.) in THF $(3 \mathrm{~mL} / \mathrm{mmol})$ was added dropwise. The reaction was allowed to warm to rt and stirred overnight. Upon completion, the reaction was quenched by addition of a saturated aqueous solution of $\mathrm{NaHCO}_{3}(10 \mathrm{~mL} / \mathrm{mmol})$. The aqueous layer was extracted with EtOAc $(2 \times 10 \mathrm{~mL} / \mathrm{mmol})$. The combined organic layers were dried over anhydrous $\mathrm{MgSO}_{4}$ and concentrated under reduced pressure. The crude was purified by flash column chromatography over silica gel (PE/EtOAc).

General procedure B for the Horner-Wadsworth-Emmons/N-methylimidazole addition sequence

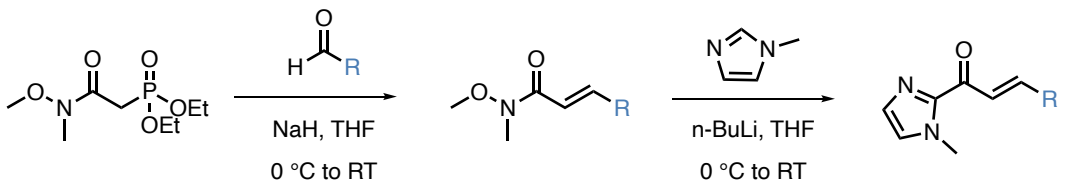

Sodium hydride (60\% dispersion in mineral oil, 1.2 equiv.) was suspended in THF ( $3 \mathrm{~mL} / \mathrm{mmol})$. A solution of phosphonate ii (1.2 equiv.) in THF (1 mL/mmol) was added dropwise at $0{ }^{\circ} \mathrm{C}$ (exothermic reaction) until gas evolution had ceased. The mixture was allowed to warm to $\mathrm{rt}$ and stirred for $1 \mathrm{~h}$ before the addition of the desired aldehyde (1.0 equiv.). The reaction mixture was stirred overnight under vigorous stirring. A saturated aqueous solution of $\mathrm{NaHCO}_{3}$ was added $(5 \mathrm{~mL} / \mathrm{mmol})$, the reaction mixture was diluted with $\mathrm{Et}_{2} \mathrm{O}(5 \mathrm{~mL} / \mathrm{mmol})$, the layers were separated and the aqueous layer was extracted with $\mathrm{Et}_{2} \mathrm{O}(2 \times 5 \mathrm{~mL})$. The combined organic layers were washed with a saturated aqueous 
solution of $\mathrm{NaCl}$, dried over anhydrous $\mathrm{MgSO}_{4}$ and concentrated under reduced pressure. The crude was purified by flash column chromatography over silica gel ( $\left.\mathrm{PE} / \mathrm{Et}_{2} \mathrm{O}\right)$. To a solution of $N$-methylimidazole (2.0 equiv.) in THF $(5 \mathrm{~mL} / \mathrm{mmol})$ was added $n$-BuLi (2.5 $\mathrm{M}$ solution in hexanes, 2.0 equiv.) dropwise at $0{ }^{\circ} \mathrm{C}$. The reaction was stirred for $20 \mathrm{~min}$, then cooled to $-78{ }^{\circ} \mathrm{C}$. The desired Weinreb amide (1.1 equiv.) was added dropwise and the mixture was stirred for $1 \mathrm{~h}$ at this temperature. Upon completion, the reaction was quenched by addition of a saturated aqueous solution of $\mathrm{NH}_{4} \mathrm{Cl}$. The aqueous phase was extracted with EtOAc $(3 \times 5 \mathrm{~mL} / \mathrm{mmol})$. The combined organic layers were washed with a saturated aqueous solution of $\mathrm{NaCl}$, dried over anhydrous $\mathrm{MgSO}_{4}$ and concentrated under reduced pressure. The crude was purified by flash column chromatography over silica gel (PE/EtOAC).

\section{General procedure $\mathbf{C}$ for the Wittig olefination}

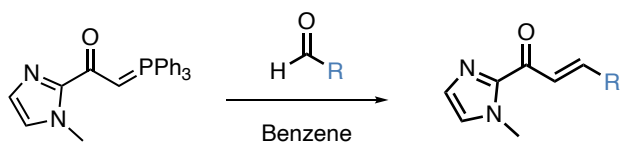

$\Delta$

To a solution of phosphaneylidene iv (1.0 equiv.) in benzene $(10 \mathrm{~mL} / \mathrm{mmol})$ were added the desired aldehyde (1.1 to 2 equiv.). The reaction mixture was stirred at reflux until TLC indicated completion (from $5 \mathrm{~h}$ to several days). Upon completion, the reaction mixture was concentrated and purified by flash column chromatography over silica gel. The pure product, if obtained with a $\mathrm{E} / \mathrm{Z}$ ratio $<95: 5$ was dissolved in dry DCM. DMAP (1 equiv.) was added and the resulting solution was stored at $-20{ }^{\circ} \mathrm{C}$ for 2 days, after which the mixture was washed with $\mathrm{HCl}(1 \mathrm{~N}$ solution) and brine. The organic layer was dried over anhydrous $\mathrm{MgSO}_{4}$ concentrated under reduced pressure. The resulting enone was used without any further purification. 
General procedure $\mathrm{D}$ for the addition of $\boldsymbol{N}$-methylimidazole to aldehydes

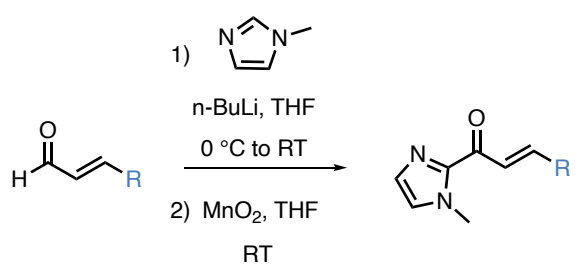

To a solution of $N$-methylimidazole (1.0 equiv.) in THF (5 mL/mmol) was added $n$-BuLi (2.5 $\mathrm{M}$ solution in hexanes, 1.1 equiv.) dropwise at $0{ }^{\circ} \mathrm{C}$. The reaction was stirred for $20 \mathrm{~min}$, then cooled to $-78{ }^{\circ} \mathrm{C}$. The desired aldehyde (1.1 equiv.) was added dropwise and the mixture was stirred for $1 \mathrm{~h}$ at this temperature. Upon completion, the reaction was quenched by addition of a saturated aqueous solution of $\mathrm{NH}_{4} \mathrm{Cl}$. The aqueous phase was extracted with EtOAc $(3 \times 5 \mathrm{~mL} / \mathrm{mmol})$. The combined organic layers were washed with a saturated aqueous solution of $\mathrm{NaCl}$, dried over anhydrous $\mathrm{MgSO}_{4}$ and concentrated under reduced pressure. The crude was purified by flash column chromatography over silica gel (PE/EtOAc). The desired alcohol was dissolved in THF $(10 \mathrm{~mL} / \mathrm{mmol})$ and manganese(IV) oxide (10 equiv.) was added. The reaction mixture was stirred for $8 \mathrm{~h}$. Upon completion, the reaction mixture was filtered over a pad of Celite (EtOAc) and concentrated under reduced pressure. The crude was purified by flash column chromatography over silica gel (PE/EtOAc).

\section{General procedure E for the aldolisation with $\mathrm{KOH}$}

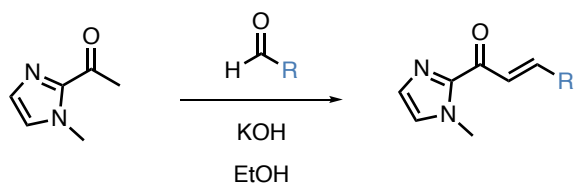

To a solution of methylketone $\mathbf{i}$ (1.0 equiv.) in ethanol $(2 \mathrm{~mL} / \mathrm{mmol}$ ) were added the desired aldehyde (1.0 equiv.) followed by potassium hydroxide (20 mol\%). The reaction mixture was stirred until TLC indicated completion (from $1 \mathrm{~h}$ to several days). The product was isolated either by direct filtration of the precipitate, which was washed with ethanol and hexane, or by addition of water $(5 \mathrm{~mL} / \mathrm{mmol})$ and extraction with EtOAc $(3 \times 10 \mathrm{~mL} / \mathrm{mmol})$, drying of the combined organic layers over anhydrous $\mathrm{MgSO}_{4}$, concentration under reduced pressure and purification by flash column chromatography over silica gel. 


\section{1-(1-Methyl-1H-imidazol-2-yl)ethan-1-one (i)}<smiles>CC(=O)c1nccn1C</smiles>

Molecular formula: $\mathrm{C}_{6} \mathrm{H}_{8} \mathrm{~N}_{2} \mathrm{O}$

$\mathrm{MW}=124.14 \mathrm{~g} \cdot \mathrm{mol}^{-1}$

To a solution of $N$-methylimidazole $(9.3 \mathrm{~mL}, 80 \mathrm{mmol}, 1.0$ equiv.) in THF (50 mL) was added $n$-BuLi (2.5 M solution in hexanes, $35.2 \mathrm{~mL}, 88 \mathrm{mmol}, 1.1$ equiv.) dropwise at $0{ }^{\circ} \mathrm{C}$. The reaction mixture was stirred for $20 \mathrm{~min}$, then transferred via cannula to a solution of 4-acetylmorpholine in $\operatorname{THF}(50 \mathrm{~mL})$ at $-78^{\circ} \mathrm{C}$. The resulting mixture was allowed to warm to $\mathrm{rt}$ and stirred for a further $16 \mathrm{~h}$. The reaction was then quenched by addition of a $3 \mathrm{M}$ aqueous solution of $\mathrm{HCl}(3.3 \mathrm{~mL})$, diluted with additional water and the aqueous phase was extracted with EtOAc $(3 \times 100 \mathrm{~mL})$. The combined organic layers were washed with a saturated aqueous solution of $\mathrm{NaCl}$, dried over anhydrous $\mathrm{MgSO}_{4}$ and concentrated under reduced pressure. Purification by flash column chromatography over silica gel $(\mathrm{PE} /$ EtOAc $=1: 1)$ afforded the desired product as a colourless oil $(7.4 \mathrm{~g}, 59 \mathrm{mmol}, 74 \%)$. Spectral data matched those reported in the literature. ${ }^{1}$

${ }^{1} \mathrm{H}$ NMR $\left(400 \mathrm{MHz}, \mathrm{CDCl}_{3}\right) \delta 7.11(\mathrm{~s}, 1 \mathrm{H}), 7.00(\mathrm{~s}, 1 \mathrm{H}), 3.98(\mathrm{~s}, 3 \mathrm{H}), 2.64(\mathrm{~s}, 3 \mathrm{H})$.

${ }^{13} \mathrm{C}$ NMR $\left(101 \mathrm{MHz}, \mathrm{CDCl}_{3}\right) \delta 190.5,143.1,128.9,126.8,36.1,27.1$.

\section{Diethyl (2-(methoxy(methyl)amino)-2-oxoethyl)phosphonate (ii)}<smiles>CCOP(=O)(CC(=O)N(C)OC)OCC</smiles>

Molecular formula: $\mathrm{C}_{8} \mathrm{H}_{18} \mathrm{NO}_{5} \mathrm{P}$

$\mathbf{M W}=139.21 \mathrm{~g} \cdot \mathrm{mol}^{-1}$

Triethyl phosphonoacetate $(29.7 \mathrm{~mL}, 150 \mathrm{mmol}, 1.0$ equiv.) in stirred in an aqueous solution of $\mathrm{NaOH}(1 \mathrm{M}, 150 \mathrm{~mL})$ for $5 \mathrm{~h}$ at $80{ }^{\circ} \mathrm{C}$ using a heating mantle. After cooling to $\mathrm{rt}$, the

\footnotetext{
${ }^{1}$ Wang, J.; Benedetti, E.; Bethge, L.; Vonhoff, S.; Klussmann, S.; Vasseur, J.-J.; Cossy, J.; Smietana, M.; Arseniyadis, S. Angew. Chem., Int. Ed. 2013, 52, 11546-11549.
} 
resulting mixture layer was acidified by slow addition of a $12 \mathrm{M}$ aqueous solution of $\mathrm{HCl}$ until $\mathrm{pH}=1(c a .12 \mathrm{~mL})$ then extracted with ethyl acetate $(5 \times 150 \mathrm{~mL})$ and concentrated under vacuum (no brine or drying agent was used as complexation of the intermediate occurs). The resulting yellow oil was dissolved in $\mathrm{CH}_{2} \mathrm{Cl}_{2}(300 \mathrm{~mL})$ and $N, N^{\prime}-$ carbonyldiimidazole $(22.3 \mathrm{~g}, 138 \mathrm{mmol}, 1.1$ equiv.) was added portion wise (resulting in quick and strong $\mathrm{CO}_{2}$ formation). The mixture was stirred until gas evolution ceased, then N,O-dimethyl hydroxylamine hydrochloride (13.4 g, $138 \mathrm{mmol}, 1.1$ equiv.) was added. The resulting mixture was stirred at $\mathrm{rt}$ for $16 \mathrm{~h}$. Water $(150 \mathrm{~mL})$ was added and the aqueous layer was extracted with $\mathrm{CH}_{2} \mathrm{Cl}_{2}(300 \mathrm{~mL})$. The combined organic layers were washed with water $(2 \times 150 \mathrm{~mL})$ and concentrated under vacuum. Purification by flash column chromatography over silica gel (EtOAc/EtOH = 8:2) afforded the title compound as a pale oil (32.3 g, $135 \mathrm{mmol}, 90 \%)$. Spectral data matched those reported in the literature. ${ }^{2}$

${ }^{1} \mathrm{H}$ NMR $\left(400 \mathrm{MHz}, \mathrm{CDCl}_{3}\right) \delta 4.18(\mathrm{dqd}, J=9.6,7.1,1.6 \mathrm{~Hz}, 4 \mathrm{H}), 3.77(\mathrm{~s}, 3 \mathrm{H}), 3.21(\mathrm{~s}, 3 \mathrm{H}), 3.16$ (d, $J=22.0 \mathrm{~Hz}, 4 \mathrm{H}), 1.34(\mathrm{t}, J=7.1 \mathrm{~Hz}, 6 \mathrm{H})$.

${ }^{13} \mathrm{C}$ NMR $\left(101 \mathrm{MHz}, \mathrm{CDCl}_{3}\right) \delta 166.3,62.7,62.6,61.6,32.3,30.9,16.5,16.5$.

\section{2-Chloro-1-(1-methyl-1H-imidazol-2-yl)ethan-1-one (iii)}

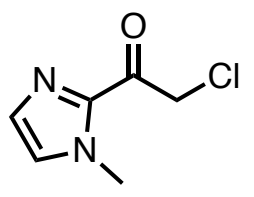

Molecular formula: $\mathrm{C}_{6} \mathrm{H}_{7} \mathrm{CIN}_{2} \mathrm{O}$

$\mathrm{MW}=158.59 \mathrm{~g} \cdot \mathrm{mol}^{-1}$

To a solution of 1-methyl imidazole $(2.39 \mathrm{~mL}, 30 \mathrm{mmol}, 1.0$ equiv.) in dry THF (75 mL) was added $n$-BuLi (2.5 M in hexanes, $6.44 \mathrm{~mL}, 45 \mathrm{mmol}, 1.5$ equiv.) at $-78{ }^{\circ} \mathrm{C}$ under argon. After stirring for 10 minutes, the resulting mixture was allowed to warm up to rt and stirred for an additional 30 minutes. The solution was then cooled down to $-78{ }^{\circ} \mathrm{C}$ and tert-butyl chloroacetate $(12 \mathrm{~mL}, 30 \mathrm{mmol}, 1.0$ equiv.) was added over 10 minutes. The resulting mixture was stirred at this temperature for $3 \mathrm{~h}$. Upon completion, the reaction was quenched by the addition of $\mathrm{H}_{2} \mathrm{O}(20 \mathrm{~mL})$ and the aqueous layer was extracted with EtOAc

\footnotetext{
${ }^{2}$ Drissi-Amraoui, S.; Morin, M. S. T.; Crévisy, C.; Baslé, O.; Marcia de Figueiredo, R.; Mauduit, M.; Campagne, J.; Angew. Chem., Int. Ed. 2015, 54, 11830-11834.
} 
( $3 \times 50 \mathrm{~mL}$ ) dried over anhydrous $\mathrm{MgSO}_{4}$ and concentrated under reduced pressure. Purification by flash column chromatography over silica gel (PE/EtOAC $=1: 1)$ afforded the title compound as a pale oil $(2.99 \mathrm{~g}, 18.9 \mathrm{mmol}, 63 \%)$. Spectral data matched those reported in the literature. ${ }^{3}$

${ }^{1} \mathrm{H}$ NMR $\left(400 \mathrm{MHz}, \mathrm{CDCl}_{3}\right) \delta 7.17(\mathrm{~d}, J=0.9 \mathrm{~Hz}, 1 \mathrm{H}), 7.11(\mathrm{~d}, J=0.9 \mathrm{~Hz}, 1 \mathrm{H}), 4.94(\mathrm{~s}, 3 \mathrm{H}), 4.04$ $(\mathrm{s}, 3 \mathrm{H})$.

${ }^{13} \mathrm{C}$ NMR $\left(101 \mathrm{MHz}, \mathrm{CDCl}_{3}\right) \delta 183.0,173.3,128.9,127.7,46.6,36.1$.

\section{1-(1-Methyl-1H-imidazol-2-yl)-2-(triphenylphosphaneylidene)ethan-1-one (iv)}

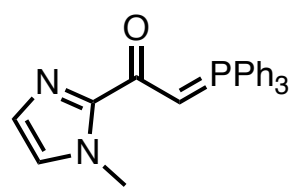

Molecular formula: $\mathrm{C}_{24} \mathrm{H}_{21} \mathrm{~N}_{2} \mathrm{OP}$

$\mathrm{MW}=384.42 \mathrm{~g} \cdot \mathrm{mol}^{-1}$

To a solution of 2-chloro-1-(1-methyl-1H-imidazol-2-yl)ethan-1-one $(2.97 \mathrm{~g}, 18.7 \mathrm{mmol}$, 1.0 equiv.) in toluene $(45 \mathrm{~mL})$ was added triphenylphosphine $(5.41 \mathrm{~g}, 20.62 \mathrm{mmol}$, 1.1 equiv.) at $\mathrm{rt}$ under argon. The resulting mixture was allowed to warm up to $95^{\circ} \mathrm{C}$ using a heating mantle. Upon completion, the solution was cooled down to rt and diluted with $\mathrm{HCl}$ $(1 \mathrm{~N}, 150 \mathrm{~mL})$ and $\mathrm{Et}_{2} \mathrm{O}(150 \mathrm{~mL})$. The aqueous layer was separated and the organic layer was extracted with $\mathrm{HCl}(1 \mathrm{~N}, 2 \times 50 \mathrm{~mL})$. The combined aqueous layers were neutralized with $\mathrm{Na}_{2} \mathrm{CO}_{3}$. The resulting aqueous layer was extracted with EtOAc $(3 \times 100 \mathrm{~mL})$, dried over anhydrous $\mathrm{MgSO}_{4}$ and concentrated. Purification by flash column chromatography over silica gel (EtOAc/MeOH/Et 3 N 9:1:0 to 8.5:1:0.5) afforded the title compound as a colourless oil $(6.94 \mathrm{~g}, 17.8 \mathrm{mmol}, 96 \%)$. Spectral data matched those reported in the literature. ${ }^{3}$

${ }^{1}$ H NMR $\left(400 \mathrm{MHz}, \mathrm{CDCl}_{3}\right) \delta$ 7.75-7.69 (m, 6H), 7.55-7.53 (m, 3H), 7.48-7.43 (m, 6H), 6.97 $(\mathrm{d}, J=1.0 \mathrm{~Hz}, 1 \mathrm{H}), 6.82(\mathrm{~s}, 1 \mathrm{H}), 4.91(\mathrm{~d}, J=24.5 \mathrm{~Hz}, 1 \mathrm{H}), 4.00(\mathrm{~s}, 3 \mathrm{H})$.

${ }^{13} \mathrm{C}$ NMR $\left(101 \mathrm{MHz}, \mathrm{CDCl}_{3}\right) \delta 177.2,146.1,133.3,133.2,132.1,132.1,128.9,128.8,127.1$, $126.9,126.2,123.5,53.1,51.9,35.8$.

${ }^{31} \mathrm{P}$ NMR $\left(162 \mathrm{MHz}, \mathrm{CDCl}_{3}\right) \delta 16.5$.

${ }^{3}$ Huo, H.; Fu, C.; Harms, K.; Meggers, E.; J. Am. Chem. Soc. 2014, 136, 2990-2993. 
Pent-4-ynal (v)

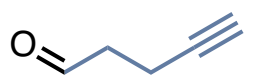

Molecular formula: $\mathrm{C}_{5} \mathrm{H}_{6} \mathrm{O}$

$\mathrm{MW}=82.10 \mathrm{~g} \cdot \mathrm{mol}^{-1}$

An oven-dried 3-neck round-bottomed flask was charged with oxalyl chloride $(1 \mathrm{~mL}$, $11.6 \mathrm{mmol}, 1.16$ equiv.) in dry $\mathrm{DCM}(25 \mathrm{~mL})$. The reaction mixture was cooled down to $-78{ }^{\circ} \mathrm{C}$. DMSO (1.6 mL, $22.0 \mathrm{mmol}, 2.2$ equiv.) in $\mathrm{DCM}(4 \mathrm{~mL})$ was slowly added and the resulting reaction mixture was stirred for 30 minutes. Petyn-1-ol (931 $\mu \mathrm{L}, 10 \mathrm{mmol}$, 1.0 equiv.) in DCM ( $2 \mathrm{~mL}$ ) was then slowly added and the mixture was allowed to stir for an additional hour, after which $\mathrm{Et}_{3} \mathrm{~N}(6.8 \mathrm{~mL}, 50 \mathrm{mmol}, 5.0$ equiv.) was slowly added and the resulting solution allowed to warm up to rt and stirred overnight. After overnight stirring, $\mathrm{H}_{2} \mathrm{O}(5 \mathrm{~mL})$ was added to the reaction mixture. The layers were separated and the aqueous layer was acidified with $\mathrm{HCl}(1 \mathrm{~N}$ solution) and extracted with $\mathrm{DCM}(2 \times 25 \mathrm{~mL})$. The combined organic layers were washed with $\mathrm{HCl}(1 \mathrm{~N}$ solution, $1 \times 10 \mathrm{~mL})$, brine $(1 \times 10 \mathrm{~mL})$, and with $\mathrm{NaHCO}_{3}(5 \%, 1 \times 10 \mathrm{~mL})$. The resulting organic layer was dried over anhydrous $\mathrm{MgSO}_{4}$ and concentrated to yield the title compound as a pale yellow oil $(616 \mathrm{mg}, 7.5 \mathrm{mmol}$, $75 \%$ yield) used without any further purification. Spectral data matched those reported in the literature. ${ }^{4}$

${ }^{1} \mathbf{H}$ NMR $\left(400 \mathrm{MHz}, \mathrm{CDCl}_{3}\right) \delta 9.80(\mathrm{~d}, J=1.2 \mathrm{~Hz}, 1 \mathrm{H}), 2.69(\mathrm{tt}, J=7.0,0.8 \mathrm{~Hz}, 2 \mathrm{H}), 2.51$ $(\mathrm{td}, J=7.1,2.8 \mathrm{~Hz}, 2 \mathrm{H}), 1.98(\mathrm{t}, J=2.8,1 \mathrm{H})$.

${ }^{13} \mathrm{C}$ NMR $\left(101 \mathrm{MHz}, \mathrm{CDCl}_{3}\right) \delta 200.0,82.3,69.3,42.4,11.7$

\footnotetext{
${ }^{4}$ Phillips, E. M.; Mesganaw, T.; Patel, A.; Duttwyler, S.; Mercado, B. Q.; Houk, K. N.; Ellman, J. A. Angew. Chem., Int. Ed., 2015, 54, 12044-12048.
} 


\section{4-[(Triisopropylsilyl)oxy]butan-1-ol (vi)}

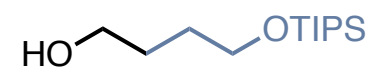

Molecular formula: $\mathrm{C}_{13} \mathrm{H}_{30} \mathrm{O}_{2} \mathrm{Si}$

$\mathrm{MW}=246.47 \mathrm{~g} \cdot \mathrm{mol}^{-1}$

To a solution of sodium hydride (740 mg, $18.5 \mathrm{mmol}, 1.24$ equiv.) in dry THF (15 mL) was slowly added at $0{ }^{\circ} \mathrm{C}$ a solution of butane-1,4-diol (1.33 mL, $15 \mathrm{mmol}, 1.0$ equiv.). The resulting solution was allowed to warm up to $\mathrm{rt}$ and stirred for 45 minutes after which triisopropylsilyl chloride (3.53 mL, $16.5 \mathrm{mmol}, 1.1$ equiv.) was added. Upon completion, the resulting mixture was partitioned between $\mathrm{Et}_{2} \mathrm{O}(120 \mathrm{~mL})$ and a saturated aqueous solution of $\mathrm{NaHCO}_{3}(60 \mathrm{~mL})$. The aqueous phase was extracted with $\mathrm{Et}_{2} \mathrm{O}(2 \times 100 \mathrm{~mL})$ and the combined organic phases were dried over anhydrous $\mathrm{MgSO}_{4}$ and concentrated to afford the title compound as a thick oil $(3.41 \mathrm{~g}, 14.6 \mathrm{mmol}, 97 \%)$. Spectral data matched those reported in the literature. ${ }^{5}$

${ }^{1} \mathrm{H}$ NMR $\left(400 \mathrm{MHz}^{\mathrm{CDCl}}{ }_{3}\right) \delta 3.75(\mathrm{dd}, J=6.4,4.7 \mathrm{~Hz}, 2 \mathrm{H}), 3.66(\mathrm{q}, J=5.5 \mathrm{~Hz}, 2 \mathrm{H}), 1.71-1.65$ $(\mathrm{m}, 2 \mathrm{H}), 1.10-1.05(\mathrm{~m}, 21 \mathrm{H})$.

${ }^{13} \mathrm{C}$ NMR $\left(101 \mathrm{MHz}, \mathrm{CDCl}_{3}\right) \delta 64.4,62.4,34.0,18.3,11.5$.

\section{4-[(Triisopropylsilyl)oxy]butanal (vii)}

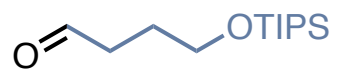

Molecular formula: $\mathrm{C}_{13} \mathrm{H}_{28} \mathrm{O}_{2} \mathrm{Si}$

$\mathrm{MW}=244.45 \mathrm{~g} \cdot \mathrm{mol}^{-1}$

To a solution of 4-[(triisopropylsilyl)oxy]butan-1-ol (697 mg, $3.0 \mathrm{mmol}, 1.0$ equiv.) in dry DCM $(10 \mathrm{~mL})$ was added freshly prepared Dess-Martin periodinane $(1.5 \mathrm{~g}, 3.6 \mathrm{mmol}$, 1.2 equiv.) in one portion at $0{ }^{\circ} \mathrm{C}$. The resulting solution was allowed to warm up to $\mathrm{rt}$ and stirred overnight. Upon completion, the reaction mixture was quenched by the addition of a saturated aqueous solution of $\mathrm{NaHCO}_{3}(5 \mathrm{~mL})$. The aqueous phase was extracted with DCM $(2 \times 20 \mathrm{~mL})$ and the combined organic phases were dried over anhydrous $\mathrm{MgSO}_{4}$ and

\footnotetext{
${ }^{5}$ Diaz, L. C.; de Oliveira, L. G.; Vilcachagua, J. D.; Nigsch, F. Org. Lett. 2005, 70, 2225-2234.
} 
concentrated. Purification by flash column chromatography over silica gel (EtOAc/EtOH = 9:1) afforded the title compound as a colourless oil $(553 \mathrm{mg}, 2.25 \mathrm{mmol}$, 75\%). Spectral data matched those reported in the literature. ${ }^{6}$

${ }^{1}$ H NMR $\left(400 \mathrm{MHz}_{\mathrm{CDCl}}\right) \delta 9.81(\mathrm{t}, J=1.7 \mathrm{~Hz}, 1 \mathrm{H}), 3.74(\mathrm{t}, J=6.0 \mathrm{~Hz}, 2 \mathrm{H}), 2.54(\mathrm{t}, J=7.1$, $1.7 \mathrm{~Hz}, 2 \mathrm{H}), 1.88(\mathrm{tt}, J=7.2,5.9 \mathrm{~Hz}, 2 \mathrm{H}), 1.09-1.02(\mathrm{~m}, 21 \mathrm{H})$.

${ }^{13} \mathrm{C}$ NMR $\left(101 \mathrm{MHz}, \mathrm{CDCl}_{3}\right) \delta 202.2,62.4,40.8,25.7,18.0,11.9$.

(E)-4,4,4-Trifluoro-N-methoxy-N-methylbut-2-enamide (viii)<smiles>CON(C)C(=O)/C=C/C(F)(F)F</smiles>

Molecular formula: $\mathrm{C}_{6} \mathrm{H}_{8} \mathrm{~F}_{3} \mathrm{NO}_{2}$

$\mathbf{M W}=183.13 \mathrm{~g} \cdot \mathrm{mol}^{-1}$

To a solution of $(E)$-4,4,4-trifluorobut-2-enoate $(1.8 \mathrm{~g}, 12.85 \mathrm{mmol}, 1.0$ equiv.) in dry DCM (45 mL) was added at $0{ }^{\circ} \mathrm{C}$ 1-ethyl-(3-(3-dimethylamino)propyl)-carbodiimide hydrochloride (EDC) (3.01 g, $15.68 \mathrm{mmol}, 1.22$ equiv.), N,O-dimethylhydroxylamine hydrochloride (1.63 g, $16.71 \mathrm{mmol}, 1.3$ equiv.) and DIPEA (3.58 mL, $21.07 \mathrm{mmol}, 1.64$ equiv.). The resulting solution was allowed to warm up to rt and stirred overnight. Upon completion, the resulting mixture was washed with brine $(20 \mathrm{~mL})$, dried over anhydrous $\mathrm{MgSO}_{4}$ and concentrated. Purification by flash column chromatography over silica gel (PE/EtOAc $=8: 2)$ afforded the title compound as a clear oil $(1.6 \mathrm{~g}, 8.74 \mathrm{mmol}, 68 \%)$. Spectral data matched those reported in the literature. ${ }^{7}$

${ }^{1} \mathbf{H}$ NMR $\left(400 \mathrm{MHz}, \mathrm{CDCl}_{3}\right) \delta 7.08(\mathrm{dd}, J=15.5,2.3 \mathrm{~Hz}, 2 \mathrm{H}), 6.82(\mathrm{dq}, J=15.6,6.7 \mathrm{~Hz}, 2 \mathrm{H})$, $3.74(\mathrm{~s}, 3 \mathrm{H}), 3.29(\mathrm{~s}, 3 \mathrm{H})$

${ }^{13} \mathrm{C}$ NMR $\left(101 \mathrm{MHz}, \mathrm{CDCl}_{3}\right) \delta 163.5,130.2(\mathrm{q}, J=35.0 \mathrm{~Hz}), 126.4(\mathrm{q}, J=6.1 \mathrm{~Hz}), 123.9,121.2$, $62.2,32.4$.

${ }^{19}$ F NMR $\left(377 \mathrm{MHz}, \mathrm{CDCl}_{3}\right) \delta-65.1(\mathrm{dd}, J=6.7,2.1 \mathrm{~Hz})$.

\footnotetext{
${ }^{6}$ Crimmins, M. T.; DeBaillie, A. C. J. Am. Chem. Soc. 2006, 128, 4936-4937.

${ }^{7}$ Yarmolchuk, V. S.; Bezdudny, A. V.; Tolmacheva, N. A.; Lukin, O.; Boyko, A. N.; Chekotylo, A.; Tolmachev, A. A.; Mykailiuk, P. K. Synthesis, 2012, 8, 1152-1154.
} 
3-(1-Methyl-1H-indol-3-yl)propanal (ix)

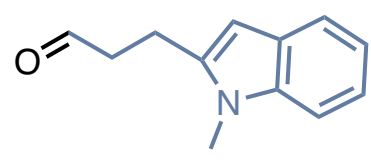

Molecular formula: $\mathrm{C}_{12} \mathrm{H}_{13} \mathrm{NO}$

$\mathrm{MW}$ (g/mol): $187.24 \mathrm{~g} \cdot \mathrm{mol}^{-1}$

To a solution of tritylium tetrafluoroborate $(46 \mathrm{mg}, 0.14 \mathrm{mmol}, 0.01$ equiv.) in dry DCM (40 mL) was added acrolein $(980 \mu \mathrm{L}, 14 \mathrm{mmol}, 1$ equiv.) and 1-methylindole (2.75 g, $21 \mathrm{mmol}, 1.5$ equiv.) The resulting solution was stirred at $\mathrm{rt}$ for $24 \mathrm{~h}$. Upon completion, the mixture was passed through a short plug of silica and concentrated. The resulting crude material was purified using flash column chromatography over silica gel. The title product was obtained as a yellow liquid $(2.68 \mathrm{~g}, 8.50 \mathrm{mmol}, 60 \%$ yield). Spectral data matched those reported in the literature. ${ }^{8}$

${ }^{1}$ H NMR (400 MHz, $\left.\mathrm{CDCl}_{3}\right) \delta 9.85(\mathrm{t}, J=1.7 \mathrm{~Hz}, 1 \mathrm{H}), 7.58(\mathrm{dt}, J=7.9,1.1 \mathrm{~Hz}, 1 \mathrm{H}), 7.30$ (dt, $J=8.3,1.0 \mathrm{~Hz}, 1 \mathrm{H}$ ), $7.23(\mathrm{dd}, J=8.2,1.3 \mathrm{~Hz}, 1 \mathrm{H}$ ), 7.12 (ddd, $J=8.0,6.9,1.1 \mathrm{~Hz}, 1 \mathrm{H}$ ), 6.85 $(\mathrm{d}, J=1.0 \mathrm{~Hz}, 1 \mathrm{H}), 3.74(\mathrm{~s}, 3 \mathrm{H}), 3.12(\mathrm{td}, J=7.3,0.8 \mathrm{~Hz}, 2 \mathrm{H}), 2.84(\mathrm{td}, J=7.3,1.8 \mathrm{~Hz}, 2 \mathrm{H})$.

${ }^{13} \mathrm{C}$ NMR (101 MHz, $\left.\mathrm{CDCl}_{3}\right) \delta$ 202.7, 137.2, 127.6, 126.5, 121.9, 119.0, 118.8, 113.2, 109.4, $44.3,32.8,17.9$.

(E)-1-(1-Methyl-1H-imidazol-2-yl)but-2-en-1-one (1a)<smiles>C/C=C/C(=O)c1nccn1C</smiles>

Molecular formula: $\mathrm{C}_{8} \mathrm{H}_{10} \mathrm{~N}_{2} \mathrm{O}$

$\mathrm{MW}(\mathrm{g} / \mathrm{mol}): 150.18 \mathrm{~g} \cdot \mathrm{mol}^{-1}$

A $500 \mathrm{~mL}$ RBF equipped with an addition funnel was charged with $\mathrm{N}$-methylimidazole (7.9 mL, $100 \mathrm{mmol}, 2.2$ equiv.) and THF $(150 \mathrm{~mL})$. The solution was cooled to $-78{ }^{\circ} \mathrm{C}$ and $n$-BuLi (2.16 M solution in hexanes, $46.1 \mathrm{~mL}, 100 \mathrm{mmol}, 2.2$ equiv.) was dropwise added. The reaction mixture was then stirred at $\mathrm{rt}$ for $60 \mathrm{~min}$. The solution was cooled to $-78{ }^{\circ} \mathrm{C}$

\footnotetext{
${ }^{8}$ Bah, J.; Naidu, V. R.; Teske, J.; Franzén, J. Adv. Synth. Catal. 2015, 357, 148-158.
} 
and a solution of crotonic acid ( $3.9 \mathrm{~g}, 45.1 \mathrm{mmol}, 1.0$ equiv.) in THF (10 mL) was dropwise added. The reaction mixture was then slowly allowed to warm up to $\mathrm{rt}$ and stirred for $16 \mathrm{~h}$. The reaction was quenched by addition of a saturated aqueous solution of $\mathrm{NaHCO}_{3}(150 \mathrm{~mL})$ and extracted with EtOAc $(4 \times 150 \mathrm{~mL})$. The combined organic layers were washed with a saturated aqueous solution of $\mathrm{NaCl}(50 \mathrm{~mL})$, dried over anhydrous $\mathrm{MgSO}_{4}$ and concentrated. The crude was purified by flash column chromatography over silica gel (PE/EtOAc $=2: 1$ to 1:1) to afford a colorless oil $(1.97 \mathrm{~g}, 13.1 \mathrm{mmol}, 29 \%)$. Spectral data matched those reported in the literature. ${ }^{1}$

${ }^{1}$ H NMR $\left(400 \mathrm{MHz}, \mathrm{CDCl}_{3}\right) \delta 7.40(\mathrm{dq}, J=15.5,1.6 \mathrm{~Hz}, 1 \mathrm{H}), 7.17-7.05(\mathrm{~m}, 2 \mathrm{H}), 7.03(\mathrm{~s}, 1 \mathrm{H})$, $4.03(\mathrm{~s}, 3 \mathrm{H}), 1.98(\mathrm{dd}, J=6.9,1.6 \mathrm{~Hz}, 3 \mathrm{H})$.

${ }^{13} \mathrm{C}$ NMR $\left(101 \mathrm{MHz}, \mathrm{CDCl}_{3}\right) \delta 180.8,144.0,143.8,129.3,127.9,127.2,36.4,18.6$.

(E)-1-(1-Methyl-1H-imidazol-2-yl)pent-2-en-1-one (1b)

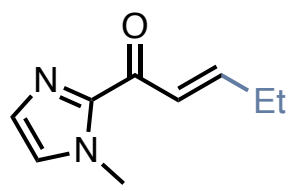

Molecular formula: $\mathrm{C}_{9} \mathrm{H}_{12} \mathrm{~N}_{2} \mathrm{O}$

$\mathrm{MW}=164.21 \mathrm{~g} \cdot \mathrm{mol}^{-1}$

The title compound was isolated as a yellow oil (949 mg, $56 \%$ yield) following general procedure D. Purified by flash column chromatography over silica gel (PE/EtOAc = 1:1). Spectral data matched those reported in the literature. ${ }^{2}$

${ }^{1}$ H NMR $\left(400 \mathrm{MHz}, \mathrm{CDCl}_{3}\right) \delta 7.39(\mathrm{dt}, J=15.7,1.6 \mathrm{~Hz}, 1 \mathrm{H}), 7.23-7.12(\mathrm{~m}, 2 \mathrm{H}), 7.03(\mathrm{~s}, 1 \mathrm{H})$, $4.04(\mathrm{~s}, 3 \mathrm{H}), 2.39-2.29(\mathrm{~m}, 2 \mathrm{H}), 1.13(\mathrm{t}, J=7.4 \mathrm{~Hz}, 3 \mathrm{H})$.

${ }^{13} \mathrm{C}$ NMR $\left(101 \mathrm{MHz}, \mathrm{CDCl}_{3}\right) \delta 180.9,150.2,143.8,129.2,127.1,125.4,36.4,25.9,12.4$. 
(E)-1-(1-Methyl-1H-imidazol-2-yl)dec-2-en-1-one (1c)<smiles>CCCCCC/C=C/C(=O)c1nccn1C</smiles>

Molecular formula: $\mathrm{C}_{14} \mathrm{H}_{22} \mathrm{~N}_{2} \mathrm{O}$

$\mathrm{MW}=234.34 \mathrm{~g} \cdot \mathrm{mol}^{-1}$

The title compound was isolated as a yellow oil (1.4 g, 65\% yield) following general procedure D. Purified by flash column chromatography over silica gel (PE/EtOAc $=3: 1$ ). Spectral data matched those reported in the literature. ${ }^{2}$

${ }^{1} \mathrm{H}$ NMR $\left(400 \mathrm{MHz}, \mathrm{CDCl}_{3}\right) \delta 7.39(\mathrm{dt}, J=15.6,1.5 \mathrm{~Hz}, 1 \mathrm{H}), 7.18-7.08(\mathrm{~m}, 2 \mathrm{H}), 7.03(\mathrm{~s}, 1 \mathrm{H})$, $4.04(\mathrm{~s}, 3 \mathrm{H}), 2.35-2.26(\mathrm{~m}, 2 \mathrm{H}), 1.57-1.46(\mathrm{~m}, 2 \mathrm{H}), 1.38-1.21(\mathrm{~m}, 8 \mathrm{H}), 0.87(\mathrm{t}, J=6.8 \mathrm{~Hz}, 3 \mathrm{H})$.

${ }^{13} \mathrm{C}$ NMR $\left(101 \mathrm{MHz}, \mathrm{CDCl}_{3}\right) \delta 180.9,149.2,143.9,129.3,127.2,126.3,36.4,32.9,31.9,29.4$, $29.2,28.4,22.8,14.2$.

(E)-5-Methyl-1-(1-methyl-1H-imidazol-2-yl)hex-2-en-1-one (1d)

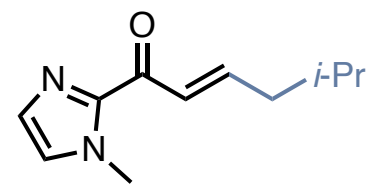

Molecular formula: $\mathrm{C}_{11} \mathrm{H}_{16} \mathrm{~N}_{2} \mathrm{O}$

$\mathrm{MW}=192.26 \mathrm{~g} \cdot \mathrm{mol}^{-1}$

The title compound was isolated as a colourless oil (125 mg, 74\% yield) following general procedure B. Purified by flash column chromatography over silica gel (PE/EtOAc = 2:1). Spectral data matched those reported in the literature. ${ }^{2}$

${ }^{1}$ H NMR $\left(400 \mathrm{MHz}, \mathrm{CDCl}_{3}\right) \delta 7.40(\mathrm{dt}, J=15.5,1.4 \mathrm{~Hz}, 1 \mathrm{H}), 7.17(\mathrm{~s}, 1 \mathrm{H}), 7.11(\mathrm{dt}, J=15.5$, $7.5 \mathrm{~Hz}, 1 \mathrm{H}), 7.04(\mathrm{~s}, 1 \mathrm{H}), 4.05(\mathrm{~s}, 3 \mathrm{H}), 2.24-2.18(\mathrm{~m}, 2 \mathrm{H}), 1.83(\mathrm{~m}, 1 \mathrm{H}), 0.95(\mathrm{~d}, J=6.8 \mathrm{~Hz}, 6 \mathrm{H})$.

${ }^{13} \mathrm{C}$ NMR $\left(101 \mathrm{MHz}, \mathrm{CDCl}_{3}\right) \delta 180.8,147.9,143.9,129.3,127.3,127.2,42.0,36.4,28.2,22.6$ (2C) 
(E)-4-Cyclohexyl-1-(1-methyl-1H-imidazol-2-yl)but-2-en-1-one (1e)

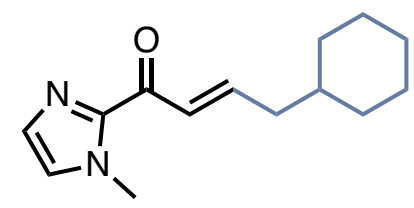

Molecular formula: $\mathrm{C}_{14} \mathrm{H}_{20} \mathrm{~N}_{2} \mathrm{O}$

$\mathrm{MW}=232.33 \mathrm{~g} \cdot \mathrm{mol}^{-1}$

The title compound was isolated as a thick yellow oil (226 mg, $0.97 \mathrm{mmol}, 68 \%$ ) using general procedure C. Purified by flash column chromatography over silica gel $(\mathrm{PE} / \mathrm{EtOAC}=3: 1)$. The DMAP treatment led to increased E/Z ratio (95:5).

${ }^{1}$ H NMR $\left(400 \mathrm{MHz}, \mathrm{CDCl}_{3}\right) \delta 7.38(\mathrm{dt}, J=15.6,1.4 \mathrm{~Hz}, 1 \mathrm{H}), 7.17(\mathrm{~d}, J=1.0 \mathrm{~Hz}, 1 \mathrm{H}), 7.12$ (dt, $J=15.4,7.6 \mathrm{~Hz}, 1 \mathrm{H}$ ), $7.03(\mathrm{~d}, J=0.9 \mathrm{~Hz}, 1 \mathrm{H}$ ), $4.04(\mathrm{~s}, 3 \mathrm{H}), 2.21$ (ddd, $J=7.8,6.8,1.4 \mathrm{~Hz}$, 2H), 1.76-1.65 (m, 4H), 1.49 (ddp, $J=13.6,10.6,3.3 \mathrm{~Hz}, 1 \mathrm{H}), 1.25-1.12(\mathrm{~m}, 4 \mathrm{H}), 1.02-0.92$ $(\mathrm{m}, 2 \mathrm{H})$.

${ }^{13} \mathrm{C}$ NMR $\left(101 \mathrm{MHz}, \mathrm{CDCl}_{3}\right) \delta 180.6,147.8,143.8,129.2,127.2,127.0,40.6,37.6,36.3,33.2$, 26.4, 26.3.

HRMS (ESI) m/z: [M+H] $]^{+}$calcd for $\mathrm{C}_{14} \mathrm{H}_{21} \mathrm{~N}_{2} \mathrm{O}$ 233.1654; found: 233.1693 .

IR (cm $\left.{ }^{-1}\right):$ 2924, 2853, 1667, 1620, 1451, 1408, 1009. 
${ }^{1} \mathrm{H}-\mathrm{NMR}$ of compound $1 \mathrm{e}\left(400 \mathrm{MHz}, \mathrm{CDCl}_{3}\right)$

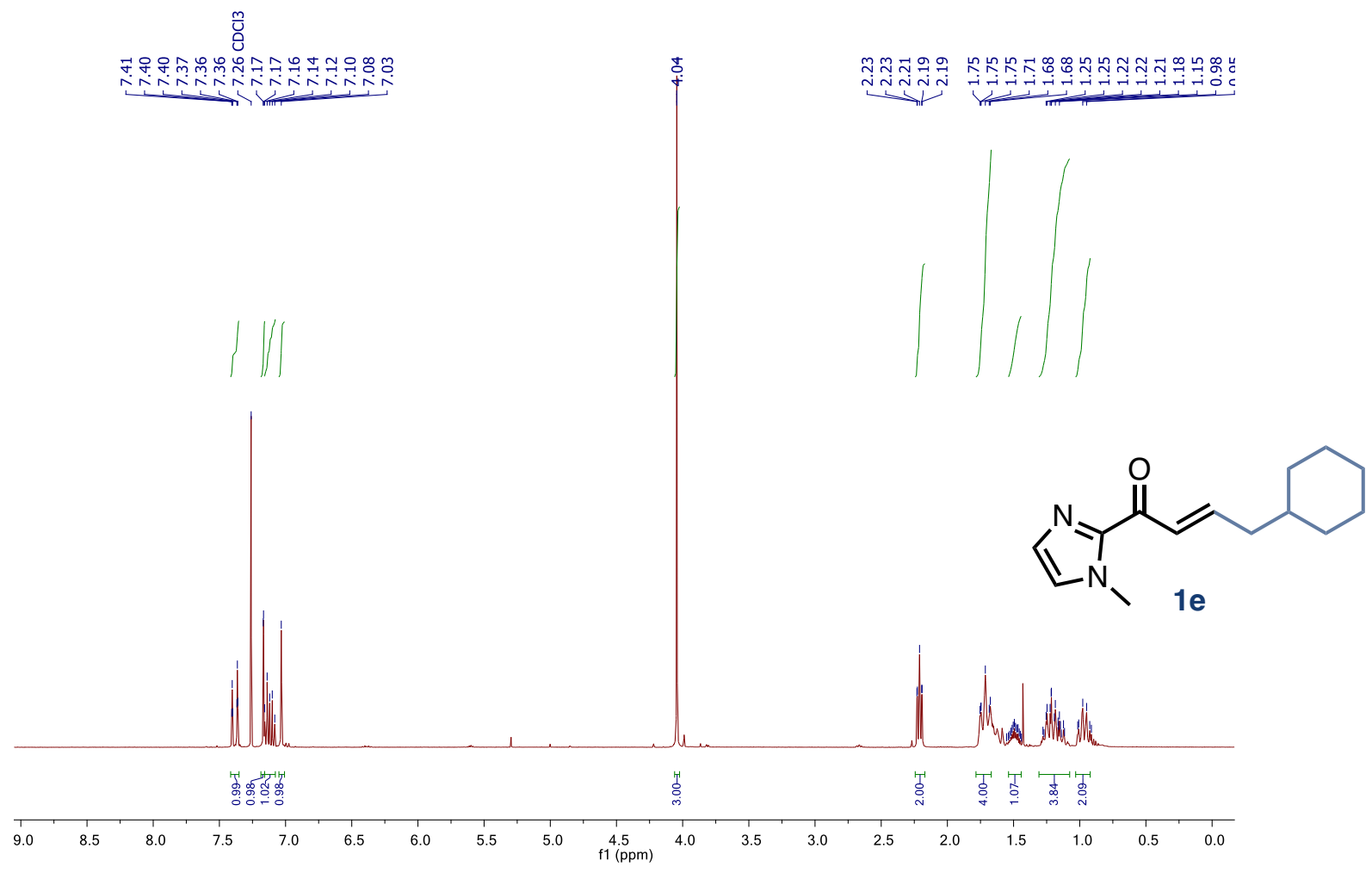

${ }^{13} \mathrm{C}-\mathrm{NMR}$ of compound $1 \mathrm{e}\left(101 \mathrm{MHz}, \mathrm{CDCl}_{3}\right)$

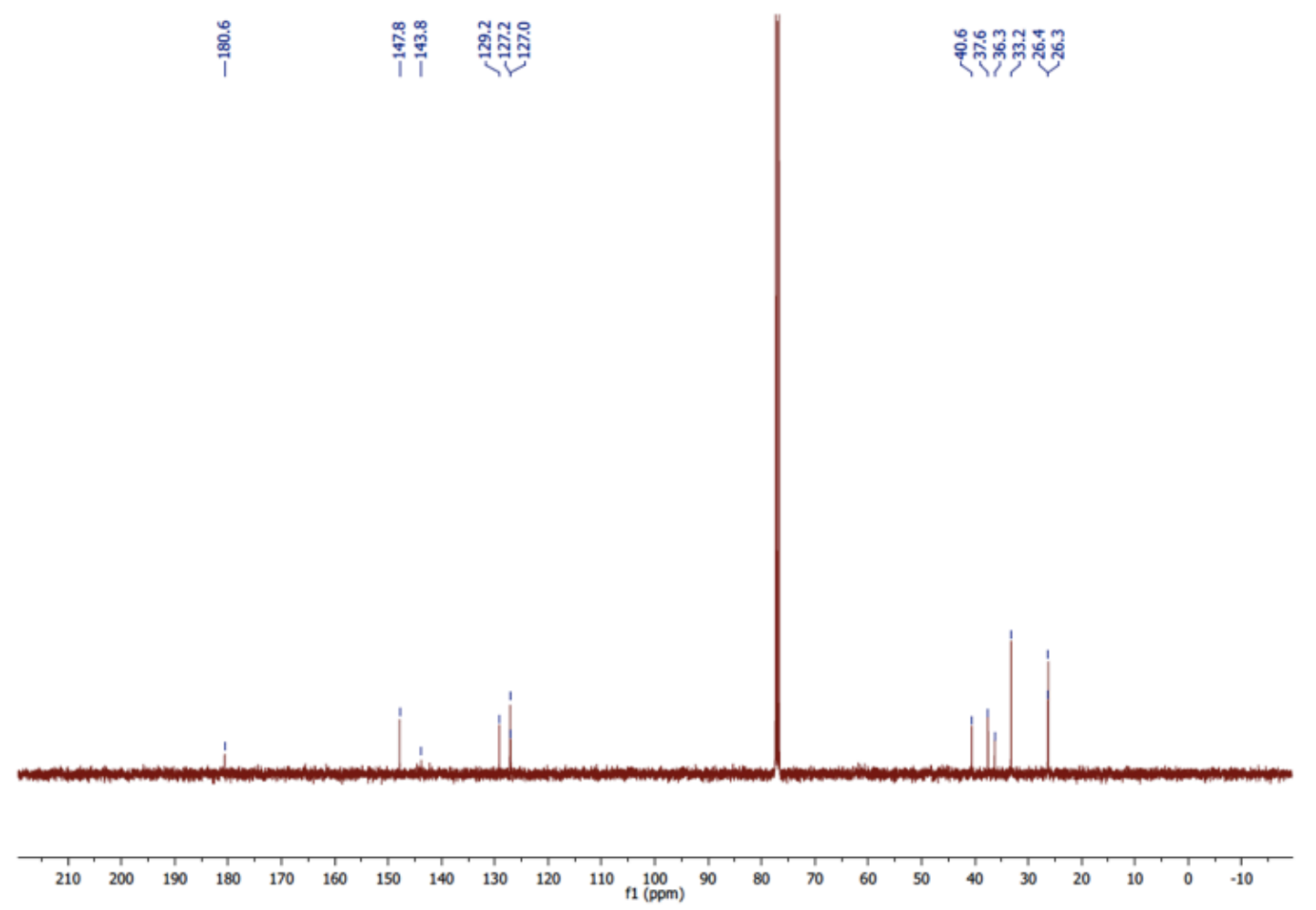


(E)-1-(1-Methyl-1H-imidazol-2-yl)-5-phenylpent-2-en-1-one (1f)

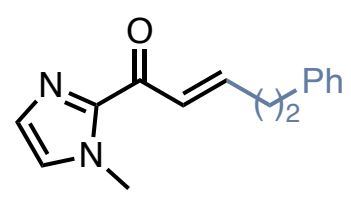

Molecular formula: $\mathrm{C}_{15} \mathrm{H}_{16} \mathrm{~N}_{2} \mathrm{O}$

$\mathbf{M W}=240.31 \mathrm{~g} \cdot \mathrm{mol}^{-1}$

The title compound was isolated as a clear oil (278 mg, $1.15 \mathrm{mmol}, 97 \%$ ) using general procedure C. Purified by flash column chromatography over silica gel (Tol/Acetone = 95:5). The DMAP treatment led to increased E/Z ratio (96:4).

${ }^{1}$ H NMR (400 MHz, $\left.\mathrm{CDCl}_{3}\right) \delta 7.59(\mathrm{dt}, J=7.9,1 \mathrm{~Hz}, 1 \mathrm{H}), 7.49(\mathrm{dt}, J=15.7,1.5 \mathrm{~Hz}, 1 \mathrm{H})$, 7.30-7.22 (m, 4H), $7.18(\mathrm{~d}, J=1.0 \mathrm{~Hz}, 1 \mathrm{H}), 7.59$ (ddd, $J=8.0,6.9,1.1 \mathrm{~Hz}, 1 \mathrm{H}), 7.04$ (d, $J=0.9 \mathrm{~Hz}, 1 \mathrm{H}), 6.87(\mathrm{~d}, J=1 \mathrm{~Hz}, 1 \mathrm{H}), 4.05(\mathrm{~s}, 3 \mathrm{H}), 3.74(\mathrm{~s}, 3 \mathrm{H}), 2.99(\mathrm{dd}, J=8.8,6.5 \mathrm{~Hz}, 2 \mathrm{H})$, $2.73(\mathrm{dtd}, J=8.8,6.5,1.5 \mathrm{~Hz}, 2 \mathrm{H})$.

${ }^{13} \mathrm{C}$ NMR $\left(101 \mathrm{MHz}, \mathrm{CDCl}_{3}\right) \delta 180.6,147.4,143.7,141.0,129.2,128.5,128.4,127.1,126.6$, 126.1, 36.3, 34.6, 34.4.

HRMS (ESI) m/z: [M+H] $]^{+}$calcd for $\mathrm{C}_{15} \mathrm{H}_{17} \mathrm{~N}_{2} \mathrm{O}$ 241.1341; found: 241.1367 .

IR $\left(\mathrm{cm}^{-1}\right):$ 3028, 2926, 1667, 1622, 1456, 1408. 


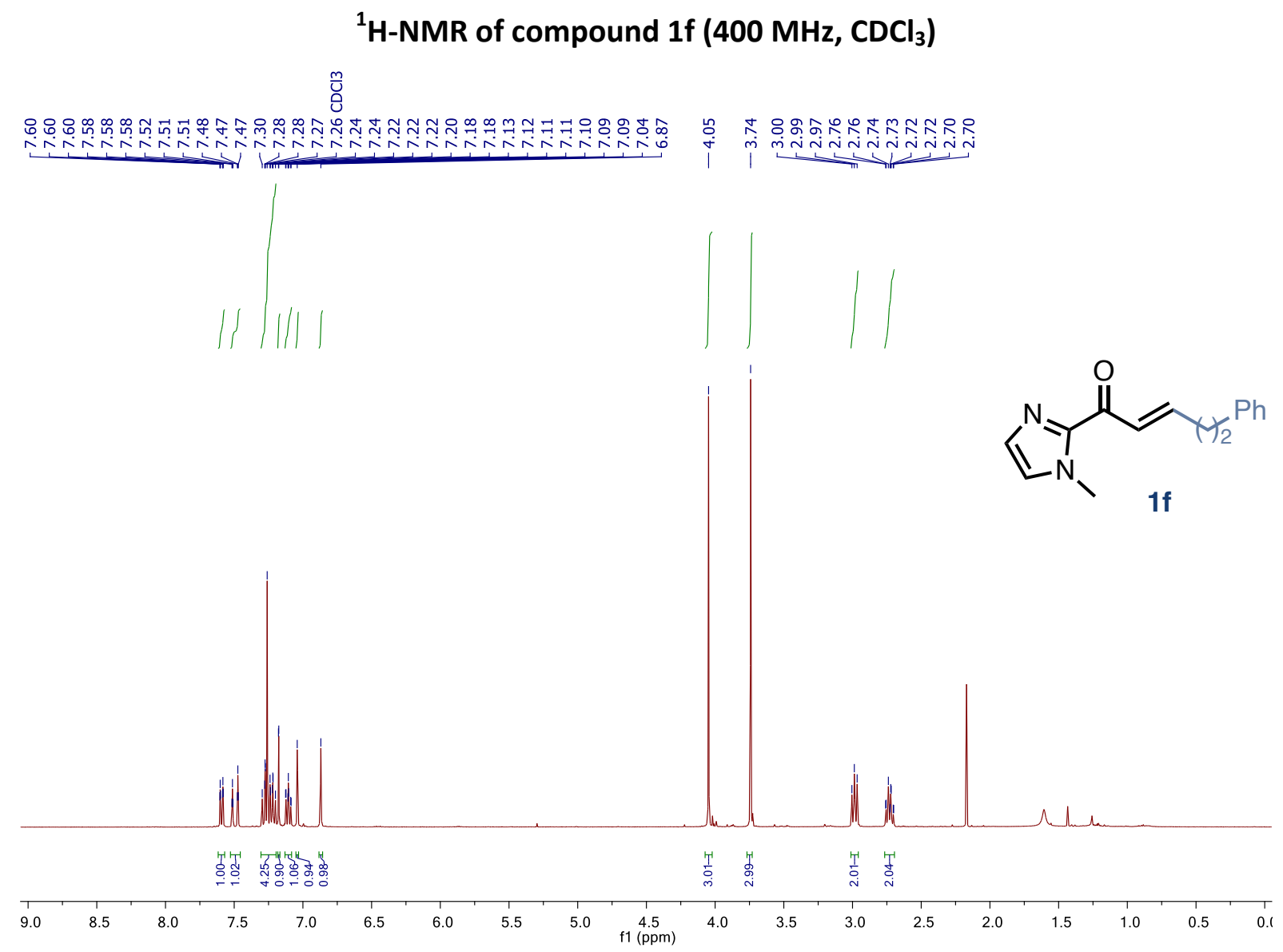

${ }^{13} \mathrm{C}-\mathrm{NMR}$ of compound if (101 $\left.\mathrm{MHz}, \mathrm{CDCl}_{3}\right)$

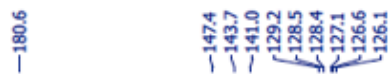

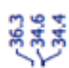

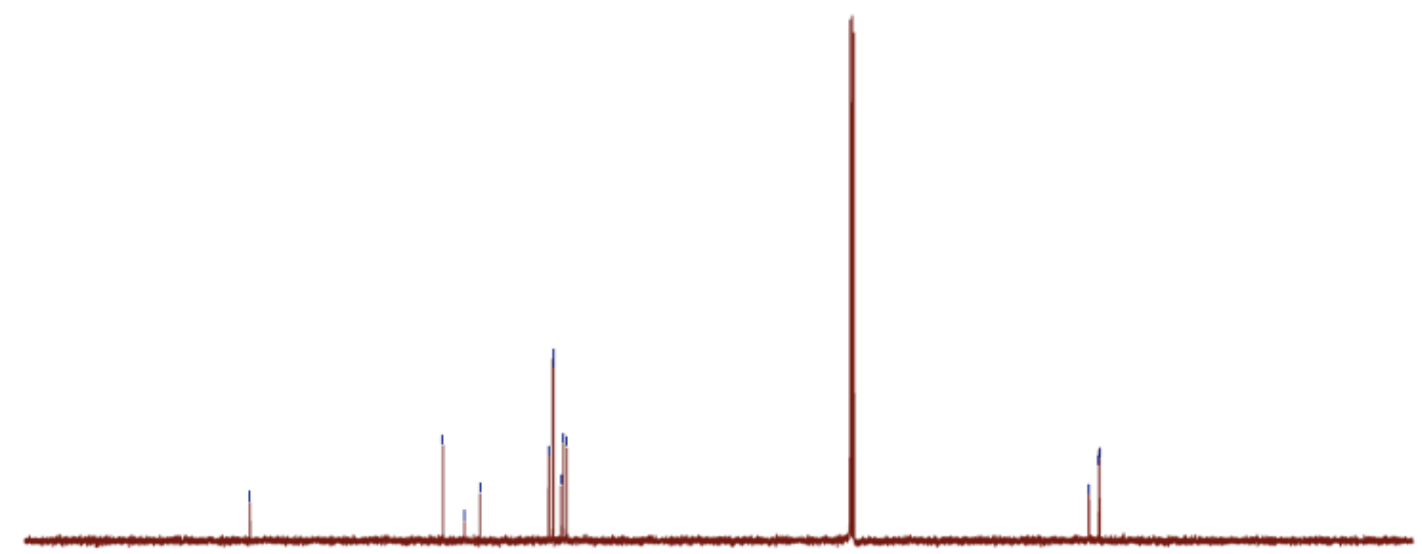

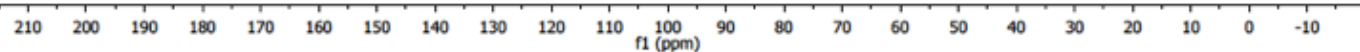


(E)-1-(1-Methyl-1H-imidazol-2-yl)hepta-2,6-dien-1-one (1g)

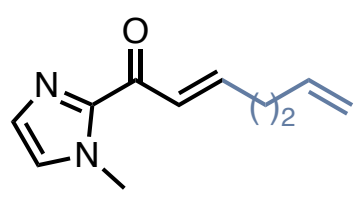

Molecular formula: $\mathrm{C}_{11} \mathrm{H}_{14} \mathrm{~N}_{2} \mathrm{O}$

$\mathbf{M W}=190.25 \mathrm{~g} \cdot \mathrm{mol}^{-1}$

The title compound was isolated as a clear oil (342 mg, $1.8 \mathrm{mmol}, 90 \%$ ) using general procedure $C$. Purified by flash column chromatography over silica gel $\left(P E / \mathrm{Et}_{2} \mathrm{O}=1: 1\right)$. The DMAP treatment led to increased $E / Z$ ratio (99:1).

${ }^{1}$ H NMR $\left(400 \mathrm{MHz}, \mathrm{CDCl}_{3}\right) \delta 7.42(\mathrm{dt}, J=15.6,1.5 \mathrm{~Hz}, 1 \mathrm{H}), 7.17(\mathrm{~s}, 1 \mathrm{H}), 7.12(\mathrm{dt}, J=15.6$, $6.8 \mathrm{~Hz}, 1 \mathrm{H}), 7.04(\mathrm{~s}, 1 \mathrm{H}), 5.83(\mathrm{ddt}, J=16.8,10.2,6.5 \mathrm{~Hz}, 2 \mathrm{H}), 5.06(\mathrm{dd}, J=17.1,1.7 \mathrm{~Hz}, 1 \mathrm{H}$ ), $5.00(\mathrm{dd}, J=10.2,1.3 \mathrm{~Hz}, 1 \mathrm{H}), 4.04(\mathrm{~s}, 3 \mathrm{H}), 2.42$ (q, $J=7.4 \mathrm{~Hz}, 2 \mathrm{H}), 2.31-2.26(\mathrm{~m}, 2 \mathrm{H}), 7.04$ (s, 1H), $4.04(\mathrm{~s}, 3 \mathrm{H}), 2.54(\mathrm{q}, J=6.8 \mathrm{~Hz}, 2 \mathrm{H}), 2.40(\mathrm{ddd}, J=7.1,2.4,1.15 \mathrm{~Hz}, 2 \mathrm{H}), 1.99$ $(\mathrm{t}, J=2.3 \mathrm{~Hz}, 1 \mathrm{H})$.

${ }^{13} \mathrm{C}$ NMR $\left(101 \mathrm{MHz}, \mathrm{CDCl}_{3}\right) \delta 180.6,147.7,143.7,137.3,129.2,127.1,126.6,155.5,36.4$, $32.3,32.0$.

HRMS (ESI) m/z: [M+H] $]^{+}$calcd for $\mathrm{C}_{11} \mathrm{H}_{15} \mathrm{~N}_{2} \mathrm{O}$ 191.1184; found: 191.1222 .

IR $\left(\mathrm{cm}^{-1}\right): 3295,2928,2363,2341,1667,1620,1462,1406,1287,1158$. 


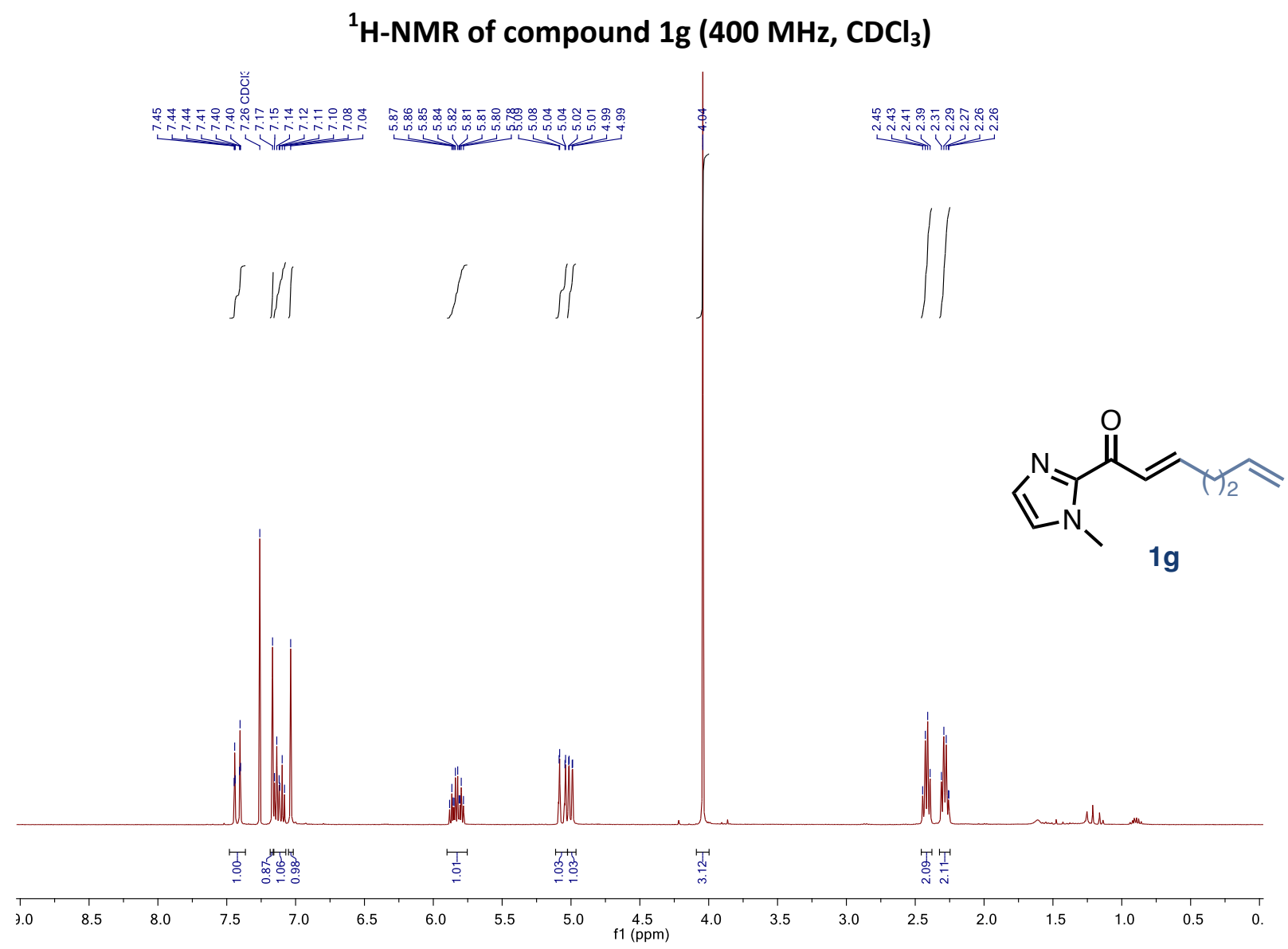

${ }^{13} \mathrm{C}-\mathrm{NMR}$ of compound $1 \mathrm{~g}\left(101 \mathrm{MHz}, \mathrm{CDCl}_{3}\right)$

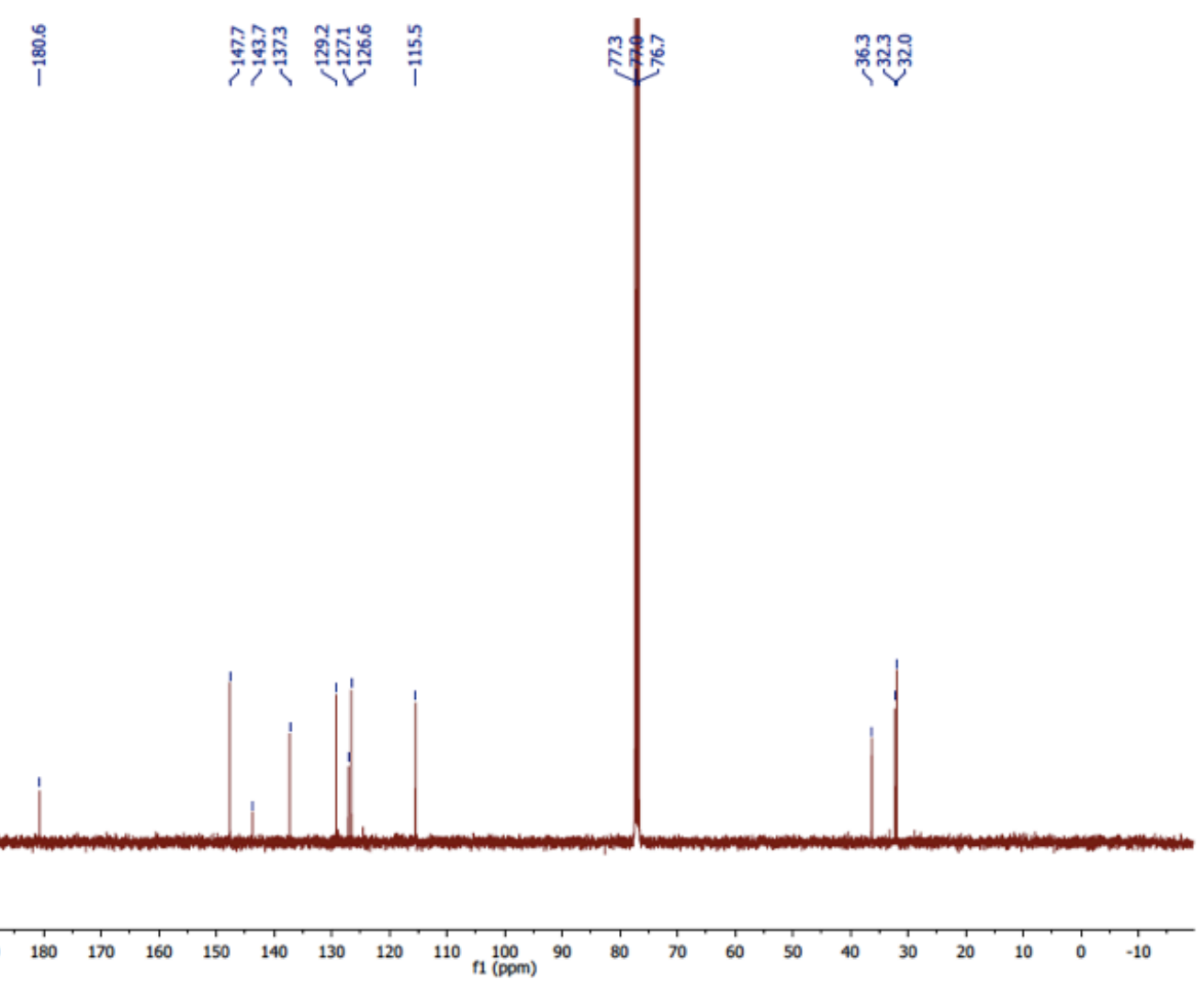

S23 
(E)-1-(1-Methyl-1H-imidazol-2-yl)hept-2-en-6-yn-1-one (1h)

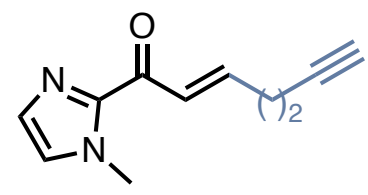

Molecular formula: $\mathrm{C}_{11} \mathrm{H}_{12} \mathrm{~N}_{2} \mathrm{O}$

$$
\mathrm{MW}=188.23 \mathrm{~g} \cdot \mathrm{mol}^{-1}
$$

The title compound was isolated as a light yellow oil (184 mg, $0.98 \mathrm{mmol}, 77 \%$ ) using general procedure C. Purified by flash column chromatography over silica gel $(\mathrm{PE} / \mathrm{EtOAC}=2: 1)$. The DMAP treatment led to increased $E / Z$ ratio $(98: 2)$.

${ }^{1}{ }^{H}$ NMR $\left(400 \mathrm{MHz} \mathrm{CDCl}_{3}\right) \delta 7.45(\mathrm{~m}, 1 \mathrm{H}), 7.17-7.08(\mathrm{~m}, 2 \mathrm{H}), 7.04(\mathrm{~s}, 1 \mathrm{H}), 4.04(\mathrm{~s}, 3 \mathrm{H}), 2.54$ (q, $J=6.8 \mathrm{~Hz}, 2 \mathrm{H}$ ), 2.40 (ddd, $J=7.1,2.4,1.15 \mathrm{~Hz}, 2 \mathrm{H}$ ), $1.99(\mathrm{t}, J=2.3 \mathrm{~Hz}, 1 \mathrm{H}$ ).

${ }^{13} \mathrm{C}$ NMR $\left(101 \mathrm{MHz}, \mathrm{CDCl}_{3}\right) \delta 180.4,145.6,143.6,129.3,127.2,82.9,69.3,36.3,31.5,17.6$. HRMS (ESI) m/z: $[\mathrm{M}+\mathrm{H}]^{+}$calcd for $\left[\mathrm{C}_{11} \mathrm{H}_{13} \mathrm{~N}_{3} \mathrm{O}\right]^{+}:$: 189.1028; found: 189.1055 .

IR $\left(\mathrm{cm}^{-1}\right): 3295,2952,1669,1624,1410$. 
${ }^{1} \mathrm{H}-\mathrm{NMR}$ of compound $1 \mathrm{~h}\left(400 \mathrm{MHz}, \mathrm{CDCl}_{3}\right)$

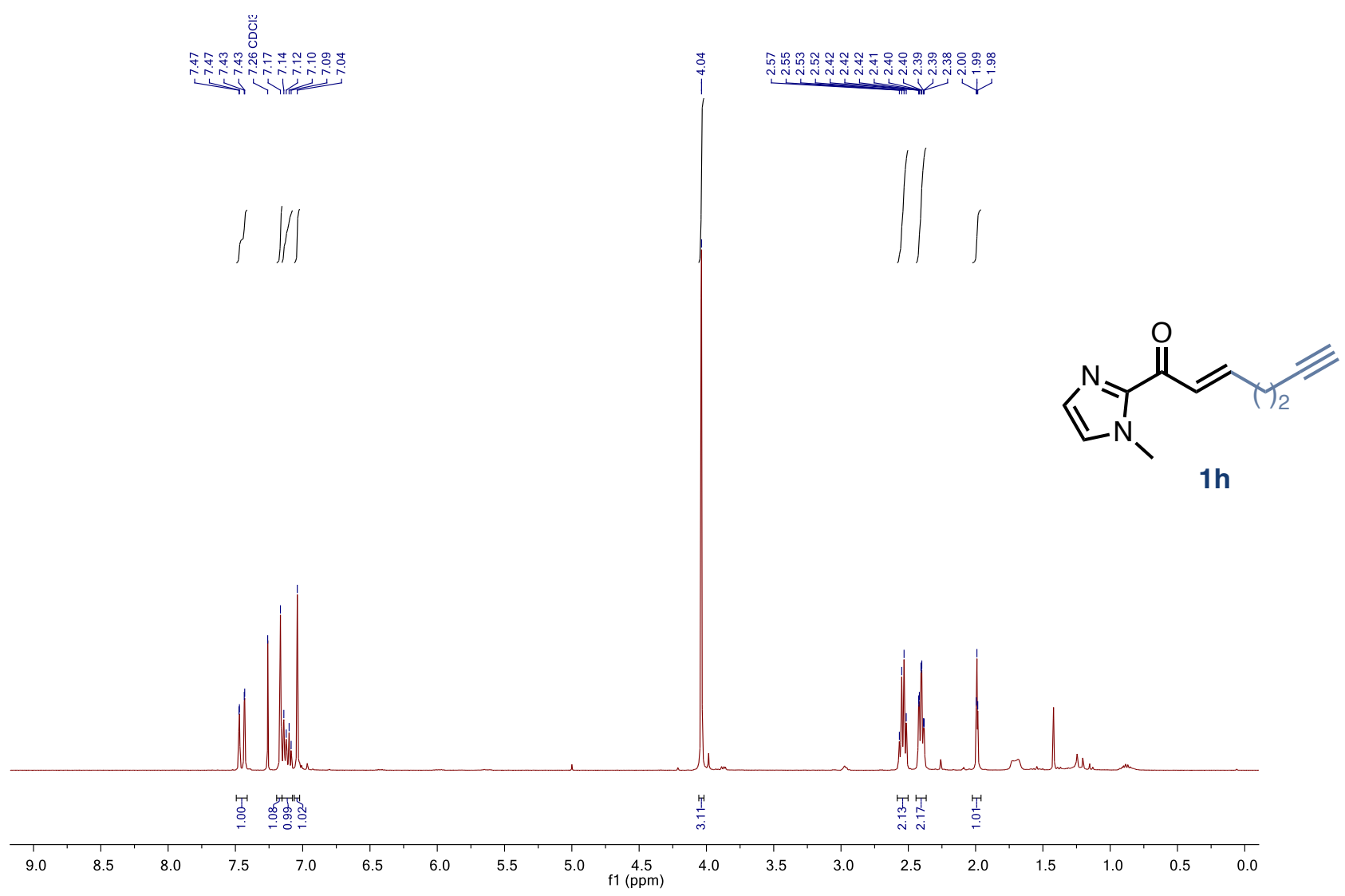

${ }^{13} \mathrm{C}-\mathrm{NMR}$ of compound $1 \mathrm{~h}\left(101 \mathrm{MHz}, \mathrm{CDCl}_{3}\right)$

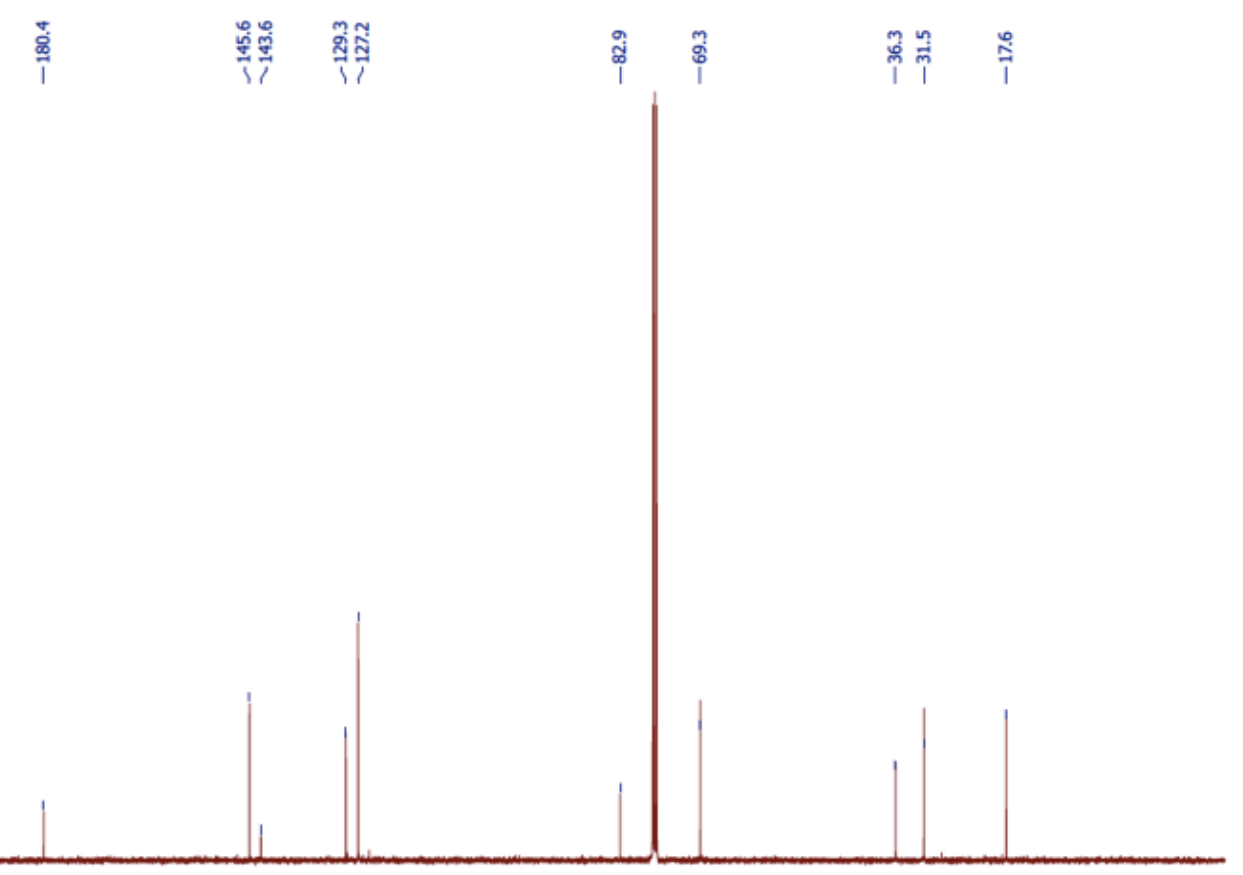

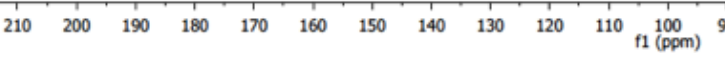

S25 
(E)-1-(1-Methyl-1H-imidazol-2-yl)-6-((triisopropylsilyl)oxy)hex-2-en-1-one (1i)

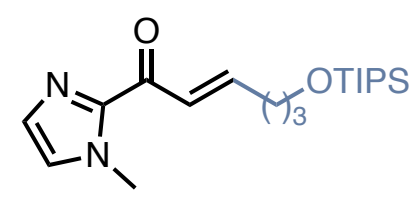

Molecular formula: $\mathrm{C}_{19} \mathrm{H}_{34} \mathrm{~N}_{2} \mathrm{O}_{2} \mathrm{Si}$

$\mathbf{M W}=350.58 \mathrm{~g} \cdot \mathrm{mol}^{-1}$

The title compound was isolated as a clear oil (232 $\mathrm{mg}, 0.66 \mathrm{mmol}, 47 \%)$ using general procedure C. Purified by flash column chromatography over silica gel (PE/EtOAC $=5: 1$ ). Spectral data matched those reported in the literature. ${ }^{9}$

${ }^{1} \mathbf{H}$ NMR $\left(400 \mathrm{MHz}, \mathrm{CDCl}_{3}\right) \delta 7.41(\mathrm{dt}, J=15.6,1.6 \mathrm{~Hz}, 1 \mathrm{H}), 7.20-7.12(\mathrm{~m}, 2 \mathrm{H}), 7.03$ (d, $J=0.9 \mathrm{~Hz}, 1 \mathrm{H}), 4.04(\mathrm{~s}, 3 \mathrm{H}), 3.73(\mathrm{t}, J=6.2 \mathrm{~Hz}, 2 \mathrm{H}), 2.42(\mathrm{dtd}, J=8.4,6.8,1.6 \mathrm{~Hz}, 2 \mathrm{H}), 1.76$ (ddt, $J=8.8,7.5,6.3 \mathrm{~Hz}, 2 \mathrm{H}), 1.07-1.04(\mathrm{~m}, 21 \mathrm{H})$.

${ }^{13} \mathrm{C}$ NMR $\left(101 \mathrm{MHz}, \mathrm{CDCl}_{3}\right) \delta 180.8,148.7,143.7,129.2,127.0,126.3,62.7,36.3,31.6,29.3$, 18.0, 12.0.

(E)-4-(Benzyloxy)-1-(1-methyl-1H-imidazol-2-yl)but-2-en-1-one (1j)<smiles>Cn1ccnc1C(=O)/C=C/COc1ccccc1</smiles>

Molecular formula: $\mathrm{C}_{15} \mathrm{H}_{16} \mathrm{~N}_{2} \mathrm{O}_{2}$

$\mathbf{M W}=256.31 \mathrm{~g} \cdot \mathrm{mol}^{-1}$

The title compound was isolated as a yellow oil (274 mg, $1.07 \mathrm{mmol}, 76 \%)$ using general procedure C. Purified by flash column chromatography over silica gel (Toluene/Acetone $=95: 5)$. Spectral data matched those reported in the literature. ${ }^{10}$

${ }^{1} \mathbf{H}$ NMR $\left(400 \mathrm{MHz}, \mathrm{CDCl}_{3}\right) \delta 7.54(\mathrm{dt}, J=15.8,1.9 \mathrm{~Hz}, 1 \mathrm{H}), 7.26-7.24(\mathrm{~m}, 2 \mathrm{H}), 7.19$ (m, 1H), $7.08(\mathrm{~d}, J=0.9 \mathrm{~Hz}, 1 \mathrm{H}), 7.04(\mathrm{dt}, J=15.8,4.8 \mathrm{~Hz}, 1 \mathrm{H}), 6.95(\mathrm{~d}, J=0.8 \mathrm{~Hz}, 1 \mathrm{H}), 4.49$ (s, $2 \mathrm{H}), 4.18(\mathrm{dd}, J=4.8,1.9 \mathrm{~Hz}, 2 \mathrm{H}), 3.9(\mathrm{~s}, 3 \mathrm{H})$.

\footnotetext{
${ }^{9}$ Tyson, E. L.; Farney, E. P.; Yoon, T. P. Org. Lett. 2012, 14, 1110-1113.
} 
${ }^{13} \mathrm{C}$ NMR $\left(101 \mathrm{MHz}, \mathrm{CDCl}_{3}\right) \delta 180.4,143.6,143.4,137.8,129.4,128.4,127.8,127.7,127.3$, $126.3,72.7,69.3,36.3$.

(E)-1-(1-Methyl-1H-imidazol-2-yl)-5-(1-methyl-1H-indol-2-yl)pent-2-en-1-one (1k)

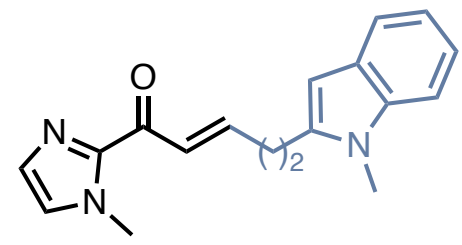

Molecular formula: $\mathrm{C}_{18} \mathrm{H}_{19} \mathrm{~N}_{3} \mathrm{O}$

$$
\mathrm{MW}=293.37 \mathrm{~g} \cdot \mathrm{mol}^{-1}
$$

The title compound was isolated as an orange powder (284 mg, $0.97 \mathrm{mmol}, 81 \%$ ) using general procedure C. Purified by flash column chromatography over silica gel $(\mathrm{PE} / \mathrm{EtOAC}=2: 1)$. Spectral data matched those reported in the literature. ${ }^{10}$

${ }^{1}$ H NMR $\left(400 \mathrm{MHz}, \mathrm{CDCl}_{3}\right) \delta 7.62(\mathrm{dt}, J=7.91,0.98 \mathrm{~Hz}, 1 \mathrm{H}), 7.52(\mathrm{dt}, J=15.7,1.5 \mathrm{~Hz}, 1 \mathrm{H})$, $7.31(\mathrm{dt}, J=8.1,0.9 \mathrm{~Hz}, 1 \mathrm{H}), 7.24(\mathrm{ddd}, J=7.9,6.9,1.2 \mathrm{~Hz}, 2 \mathrm{H}), 7.20(\mathrm{~d}, J=0.9 \mathrm{~Hz}, 1 \mathrm{H}), 7.13$ (ddd, $J=8.0,6.9,1.2 \mathrm{~Hz}, 1 \mathrm{H}$ ), 7.07 (d, J = 0.9 Hz, 1H), 6.89 (d, J = 1.0 Hz, 1H), $4.07(\mathrm{~s}, 3 \mathrm{H}$ ), $3.77(\mathrm{~s}, 3 \mathrm{H}), 3.01$ (dd, $J=8.8,6.6 \mathrm{~Hz}, 2 \mathrm{H}), 1.51$ (dtd, $J=8.8,6.8,1.5 \mathrm{~Hz}, 2 \mathrm{H}$ ).

${ }^{13} \mathrm{C}$ NMR $\left(101 \mathrm{MHz}, \mathrm{CDCl}_{3}\right) \delta 180.7,148.3,143.8,137.1,129.2,127.7,126.4,126.21,121.6$, $118.9,118.7,113.9,109.2,36.3,33.5,32.6,24.0$.

(E)-4,4,4-Trifluoro-1-(1-methyl-1H-imidazol-2-yl)but-2-en-1-one (1I)<smiles>Cn1ccnc1C(=O)/C=C/C(F)(F)F</smiles>

Molecular formula: $\mathrm{C}_{8} \mathrm{H}_{7} \mathrm{~F}_{3} \mathrm{~N}_{2} \mathrm{O}$

$\mathrm{MW}=204.15 \mathrm{~g} \cdot \mathrm{mol}^{-1}$

The title compound was obtained as yellow crystals (390 mg, $1.9 \mathrm{mmol}, 35 \%$ ) using general procedure B (second part). Purified by flash column chromatography over silica gel $(P E / E t O A C=3: 1)$. Spectral data matched those reported in the literature. ${ }^{11}$

${ }^{10}$ Park, S.; Ikehata, K.; Wanatabe, R.; Hidaka, Y.; Rajendran, A.; Sugiyama, H. Chem. Comm. 2012, 48, 1039810400. 
${ }^{1} \mathbf{H}$ NMR $\left(400 \mathrm{MHz}, \mathrm{CDCl}_{3}\right) \delta 8.03(\mathrm{dq}, J=15.8,2.0 \mathrm{~Hz}, 1 \mathrm{H}), 7.25(\mathrm{~d}, J=0.9 \mathrm{~Hz}, 1 \mathrm{H}), 7.14$ $(\mathrm{s}, 1 \mathrm{H}), 6.86(\mathrm{dq}, J=15.9,6.8 \mathrm{~Hz}, 1 \mathrm{H}), 4.07(\mathrm{~s}, 3 \mathrm{H})$.

${ }^{13} \mathrm{C}$ NMR $\left(101 \mathrm{MHz}, \mathrm{CDCl}_{3}\right) \delta 178.0,132.3,130.4,129.2,128.4,36.3$.

${ }^{19}$ F NMR $\left(377 \mathrm{MHz}, \mathrm{CDCl}_{3}\right) \delta-64.9(\mathrm{dd}, J=6.9,2.1 \mathrm{~Hz})$.

(E)-3-Cyclopropyl-1-(1-methyl-1H-imidazol-2-yl)prop-2-en-1-one (1m)<smiles>Cn1ccnc1C(=O)/C=C/C1CC1</smiles>

Molecular formula: $\mathrm{C}_{10} \mathrm{H}_{12} \mathrm{~N}_{2} \mathrm{O}$

$\mathrm{MW}=176.22 \mathrm{~g} \cdot \mathrm{mol}^{-1}$

The title compound was isolated was isolated as a yellow oil (159 mg, 56\% yield) using general procedure D. Purified by flash column chromatography over silica gel $(\mathrm{PE} / \mathrm{EtOAC}=3: 1)$.

${ }^{1} \mathbf{H}$ NMR $\left(400 \mathrm{MHz}, \mathrm{CDCl}_{3}\right) \delta 7.49(\mathrm{~d}, J=15.4 \mathrm{~Hz}, 1 \mathrm{H}), 7.16(\mathrm{~d}, J=0.9 \mathrm{~Hz}, 1 \mathrm{H}), 7.02$ (d, $J=0.9 \mathrm{~Hz}, 1 \mathrm{H}), 6.60(\mathrm{dd}, J=15.4,10.1 \mathrm{~Hz}, 1 \mathrm{H}), 4.03(\mathrm{~s}, 3 \mathrm{H}), 1.72(\mathrm{~m}, 1 \mathrm{H}), 1.05-0.96(\mathrm{~m}$, $2 \mathrm{H}), 0.78-0.68(\mathrm{~m}, 2 \mathrm{H})$.

${ }^{13} \mathrm{C}$ NMR $\left(101 \mathrm{MHz}, \mathrm{CDCl}_{3}\right) \delta 180.3,154.2,143.9,129.2,127.1,123.6,36.4,15.4,9.5$ (2C).

HRMS (ESI) m/z: [M+H] calcd for $\mathrm{C}_{10} \mathrm{H}_{13} \mathrm{~N}_{2} \mathrm{O}$ 177.1028; found: 177.1004 .

IR $\left(\mathrm{cm}^{-1}\right):$ 3010, 2959, 1663, 1611, 1467, 1411, 1266, 1032.

${ }^{11}$ Lauberteaux, J.; Crévisy, C.; Baslé, O.; de Figueiredo, R. M.; Mauduit, M.; Campagne, J.-M. Org. Lett. 2019, $21,1872-1876$. 
${ }^{1} \mathrm{H}-\mathrm{NMR}$ of compound $1 \mathrm{~m}\left(400 \mathrm{MHz}, \mathrm{CDCl}_{3}\right)$

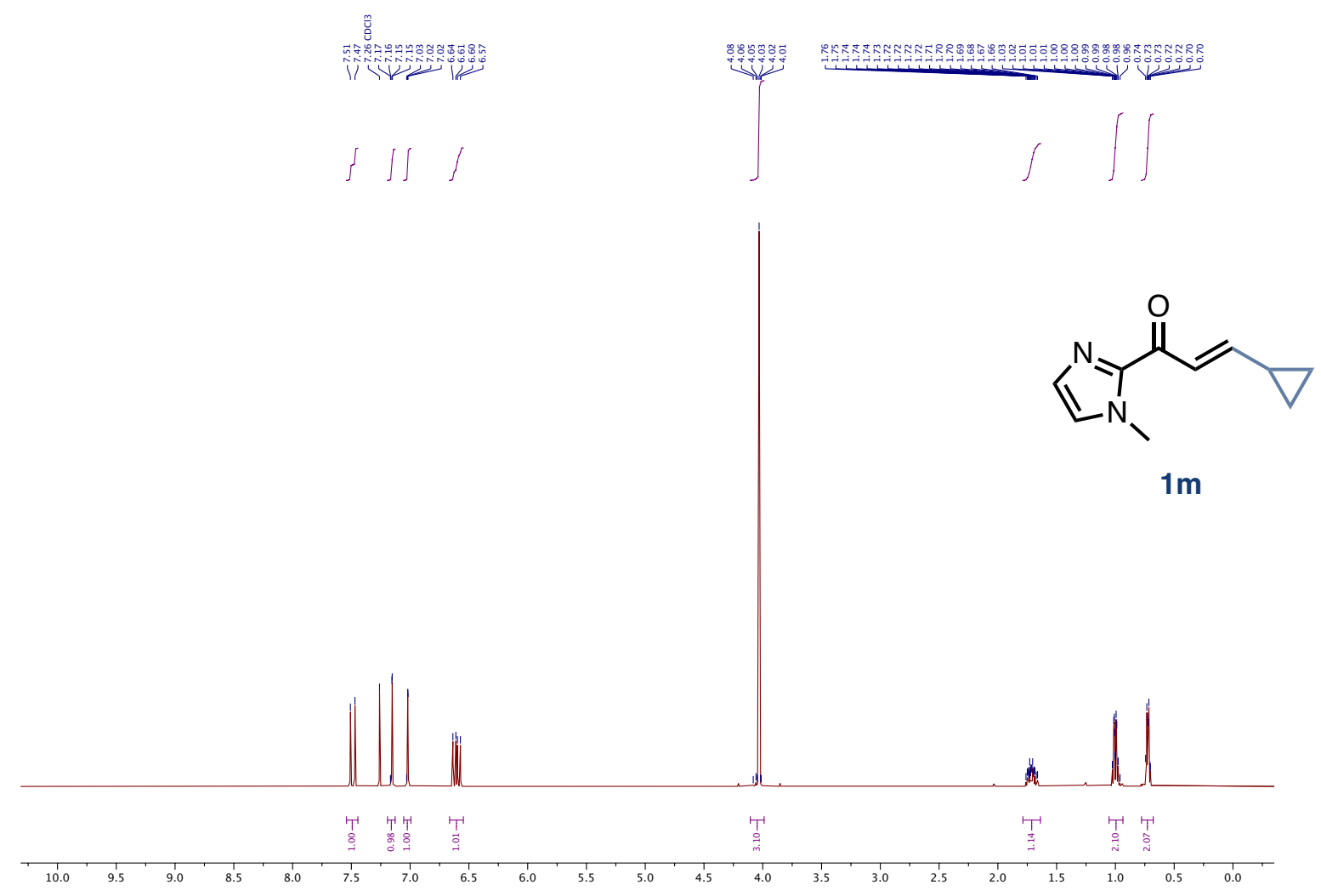

${ }^{13} \mathrm{C}-\mathrm{NMR}$ of compound $1 \mathrm{~m}\left(101 \mathrm{MHz}, \mathrm{CDCl}_{3}\right)$

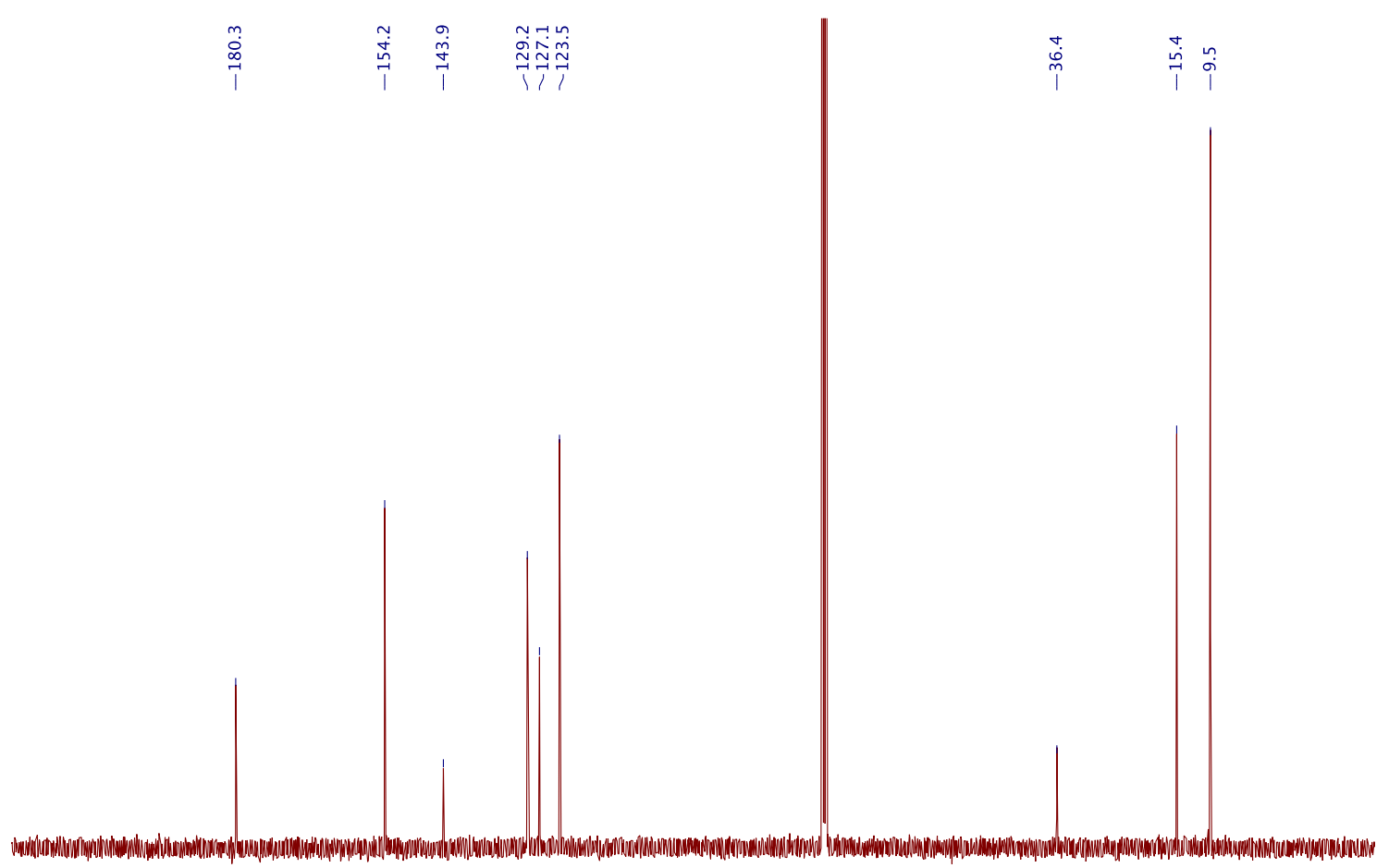

$\begin{array}{lllllllllllllllllllllll}210 & 200 & 190 & 180 & 170 & 160 & 150 & 140 & 130 & 120 & 110 & 100 & 90 & 80 & 70 & 60 & 50 & 40 & 30 & 20 & 10 & 0 & -10\end{array}$ 
(E)-4-Methyl-1-(1-methyl-1H-imidazol-2-yl)pent-2-en-1-one (1n)

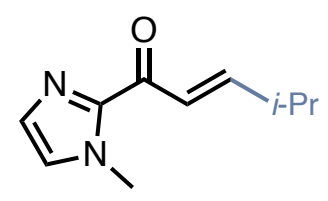

Molecular formula: $\mathrm{C}_{10} \mathrm{H}_{14} \mathrm{~N}_{2} \mathrm{O}$

$\mathbf{M W}=178.24 \mathrm{~g} \cdot \mathrm{mol}^{-1}$

The title compound was isolated as a pale oil (138 mg, 61\% yield) using general procedure $\mathbf{B}$. Purified by flash column chromatography over silica gel $(P E / E t O A c=3: 1)$. Spectral data matched those reported in the literature. ${ }^{9}$

${ }^{1}$ H NMR $\left(400 \mathrm{MHz}, \mathrm{CDCl}_{3}\right) \delta 7.35(\mathrm{dd}, J=15.7,1.4 \mathrm{~Hz}, 1 \mathrm{H}), 7.16(\mathrm{~s}, 1 \mathrm{H}), 7.09$ (dd, $J=15.7$, $6.7 \mathrm{~Hz}, 1 \mathrm{H}), 7.03(\mathrm{~s}, 1 \mathrm{H}), 4.03(\mathrm{~s}, 3 \mathrm{H}), 2.56(\mathrm{~m}, 1 \mathrm{H}), 1.12(\mathrm{~d}, J=6.8 \mathrm{~Hz}, 6 \mathrm{H})$.

${ }^{13} \mathrm{C}$ NMR $\left(101 \mathrm{MHz}, \mathrm{CDCl}_{3}\right) \delta 181.2,155.0,144.0,129.3,127.2,123.5,36.4,31.5,21.5$ (2C).

(E)-3-Cyclohexyl-1-(1-methyl-1H-imidazol-2-yl)prop-2-en-1-one (10)

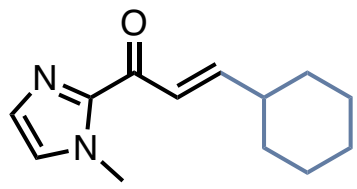

Molecular formula: $\mathrm{C}_{13} \mathrm{H}_{18} \mathrm{~N}_{2} \mathrm{O}$

$\mathbf{M W}=218.30 \mathrm{~g} \cdot \mathrm{mol}^{-1}$

The title compound was isolated as a yellow oil ( $308 \mathrm{mg}, 44 \%$ yield) using general procedure B. Purified by flash column chromatography over silica gel $\left(\mathrm{PE} / \mathrm{Et}_{2} \mathrm{O}=1: 1\right)$. Spectral data matched those reported in the literature. ${ }^{9}$

${ }^{1}$ H NMR $\left(400 \mathrm{MHz}, \mathrm{CDCl}_{3}\right) \delta 7.35(\mathrm{dd}, J=15.8,1.4 \mathrm{~Hz}, 1 \mathrm{H}), 7.16(\mathrm{~s}, 1 \mathrm{H}), 7.06(\mathrm{dd}, J=15.8$, $6.8 \mathrm{~Hz}, 1 \mathrm{H}), 7.02(\mathrm{~s}, 1 \mathrm{H}), 4.03(\mathrm{~s}, 3 \mathrm{H}), 2.24(\mathrm{~m}, 1 \mathrm{H}), 1.88-1.79(\mathrm{~m}, 2 \mathrm{H}), 1.79-1.72(\mathrm{~m}, 2 \mathrm{H}), 1.68$ (m, 1H), 1.38-1.13 (m, 5H).

${ }^{13} \mathrm{C}$ NMR $\left(101 \mathrm{MHz}, \mathrm{CDCl}_{3}\right) \delta 181.3,153.8,144.0,129.2,127.1,123.9,41.0,36.4,31.9(2 \mathrm{C})$, $26.1,25.9(2 \mathrm{C})$. 
tert-Butyl (E)-4-(3-(1-methyl-1H-imidazol-2-yl)-3-oxoprop-1-en-1-yl)piperidine-1carboxylate (1p)

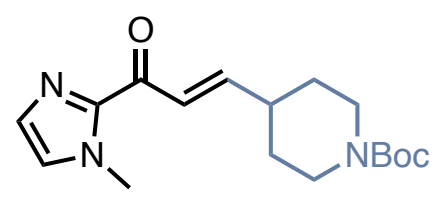

Molecular formula: $\mathrm{C}_{17} \mathrm{H}_{25} \mathrm{~N}_{3} \mathrm{O}_{3}$

$$
\mathrm{MW}=319.41 \mathrm{~g} \cdot \mathrm{mol}^{-1}
$$

The title compound was isolated as a white solid (404 mg, $1.27 \mathrm{mmol}, 90 \%$ ) using general procedure C. Purified by flash column chromatography over silica gel $(\mathrm{PE} /$ EtOAc/Acetone $=6: 3: 1)$. Spectral data matched those reported in the literature. ${ }^{9}$

${ }^{1} \mathbf{H}$ NMR $\left(400 \mathrm{MHz}, \mathrm{CDCl}_{3}\right) \delta 7.40(\mathrm{dt}, J=15.8,1.2 \mathrm{~Hz}, 1 \mathrm{H}), 7.17(\mathrm{t}, J=1.1 \mathrm{~Hz}, 1 \mathrm{H})$, 7.06-7.00(m, 2H), 4.12 (br s, 2H), 4.04 (s, 3H), 2.78 (t, J = $12.7 \mathrm{~Hz}, 2 \mathrm{H}), 2.40(\mathrm{~m}, 1 \mathrm{H}), 1.79$ (dd, $J=13.1,3.3 \mathrm{~Hz}, 2 \mathrm{H}), 1.46(\mathrm{~m}, 9 \mathrm{H}), 1.39(\mathrm{dd}, J=12.0,4.2 \mathrm{~Hz}, 1 \mathrm{H})$.

${ }^{13} \mathrm{C}$ NMR $\left(101 \mathrm{MHz}, \mathrm{CDCl}_{3}\right) \delta 180.7,154.8,150.8,143.7,129.3,127.2,124.8,79.5,39.1,36.3$, $30.8,28.5$.

(E)-4-Cyclohexyl-1-(1-methyl-1H-imidazol-2-yl)but-2-en-1-one (1q)

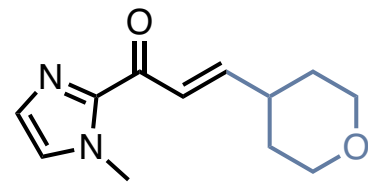

Molecular formula: $\mathrm{C}_{12} \mathrm{H}_{16} \mathrm{~N}_{2} \mathrm{O}_{2} \quad \mathrm{MW}=220.27 \mathrm{~g} \cdot \mathrm{mol}^{-1}$

The title compound was isolated as white crystals ( $273 \mathrm{mg}, 1.24 \mathrm{mmol}, 88 \%$ ) using general procedure $\mathbf{C}$. Purified by flash column chromatography over silica gel (PE/EtOAC $=3: 1$ ).

${ }^{1} \mathrm{H}$ NMR $\left(400 \mathrm{MHz}, \mathrm{CDCl}_{3}\right) \delta 7.40(\mathrm{dd}, J=15.8,1.4 \mathrm{~Hz}, 1 \mathrm{H}), 7.18(\mathrm{~d}, J=1.0 \mathrm{~Hz}, 1 \mathrm{H}), 7.07-7.02$ (m, 2H), $7.03(\mathrm{~d}, J=0.9 \mathrm{~Hz}, 1 \mathrm{H}), 4.05(\mathrm{~s}, 3 \mathrm{H}), 4.02(\mathrm{dd}, J=4.3,2.3 \mathrm{~Hz}, 1 \mathrm{H}), 3.99(\mathrm{~m}, 1 \mathrm{H}), 3.46$ (td, $J=11.6,2.3 \mathrm{~Hz}, 1 \mathrm{H}), 2.50(\mathrm{~m}, 1 \mathrm{H}), 1.76(\mathrm{~m}, 1 \mathrm{H}), 1.73(\mathrm{dd}, J=4.2,2.0 \mathrm{~Hz}, 1 \mathrm{H}), 1.66-1.56$ $(m, 2 \mathrm{H})$.

${ }^{13} \mathrm{C}$ NMR $\left(101 \mathrm{MHz}, \mathrm{CDCl}_{3}\right) \delta 180.8,151.0,129.3,127.2,124.5,67.5,38.1,36.3,31.5$.

HRMS (ESI) m/z: [M+H] $]^{+}$calcd for $\mathrm{C}_{12} \mathrm{H}_{17} \mathrm{~N}_{2} \mathrm{O}$ 221.1290; found: 221.1283 .

IR $\left(\mathrm{cm}^{-1}\right): 2935,2844,1669,1620,1466,1410,1007$. 
${ }^{1} \mathrm{H}-\mathrm{NMR}$ of compound $1 \mathrm{q}\left(400 \mathrm{MHz}, \mathrm{CDCl}_{3}\right)$

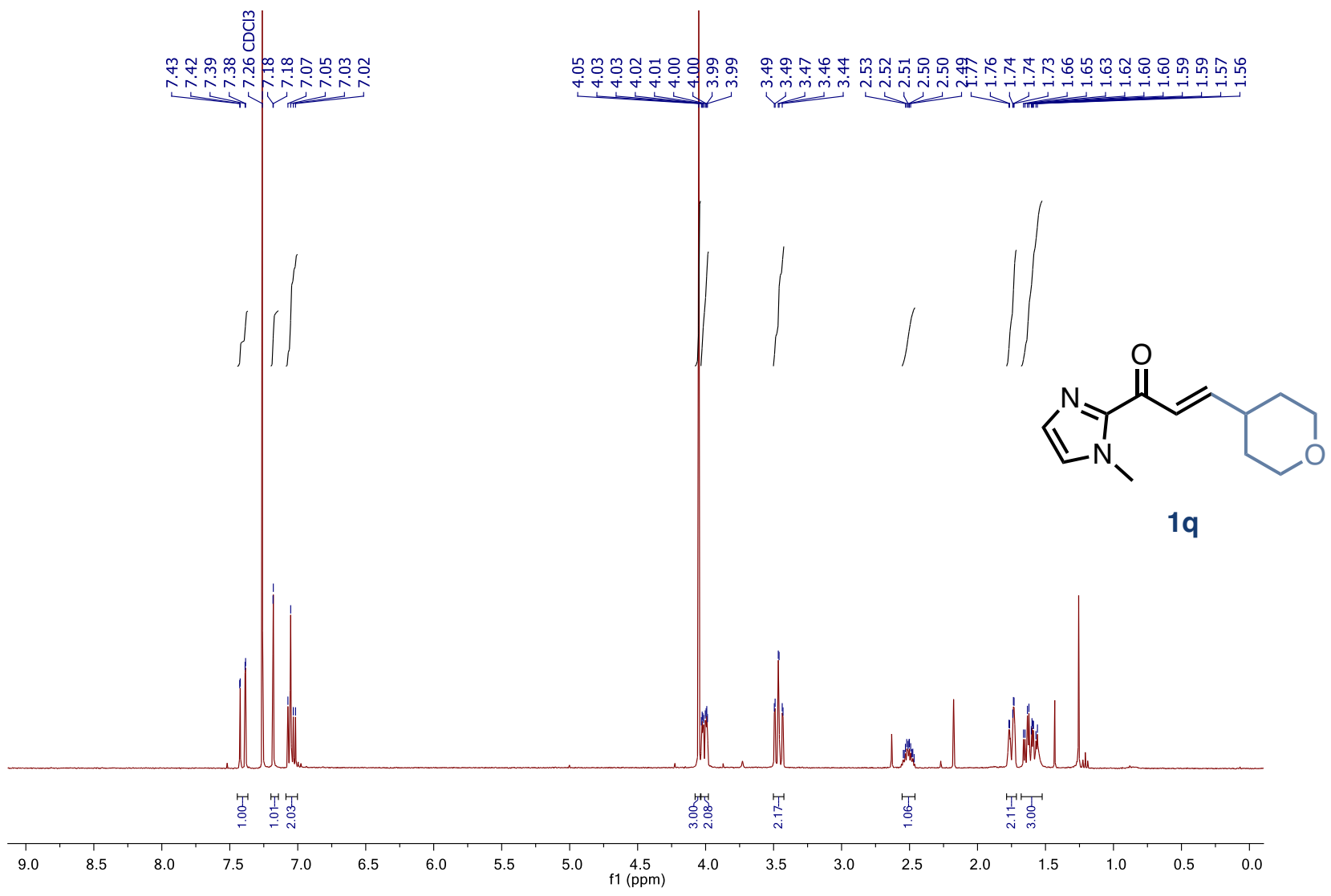

${ }^{13} \mathrm{C}-\mathrm{NMR}$ of compound $1 \mathrm{q}\left(101 \mathrm{MHz}, \mathrm{CDCl}_{3}\right)$

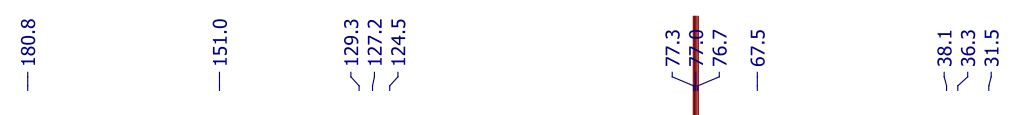




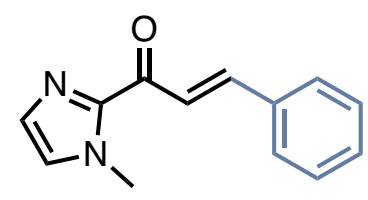

Molecular formula: $\mathrm{C}_{13} \mathrm{H}_{12} \mathrm{~N}_{2} \mathrm{O}$

$\mathbf{M W}=212.25 \mathrm{~g} \cdot \mathrm{mol}^{-1}$

The title compound was isolated as a yellow solid (856 mg, 75\% yield) using general procedure E. Purified by flash column chromatography over silica gel (PE/EtOAc $=2: 1$ ). Spectral data matched those reported in the literature. ${ }^{1}$

${ }^{1}$ H NMR (400 MHz, CDCl $) \delta 8.08(\mathrm{~d}, J=16.0 \mathrm{~Hz}, 1 \mathrm{H}), 7.83(\mathrm{~d}, J=16.0 \mathrm{~Hz}, 1 \mathrm{H}), 7.73-7.67$ (m, 2H), 7.44-7.36 (m, 3H), $7.22(\mathrm{~d}, J=0.9 \mathrm{~Hz}, 1 \mathrm{H}), 7.08(\mathrm{~d}, J=0.9 \mathrm{~Hz}, 1 \mathrm{H}), 4.10(\mathrm{~s}, 3 \mathrm{H})$.

${ }^{13} \mathrm{C}$ NMR $\left(101 \mathrm{MHz}, \mathrm{CDCl}_{3}\right) \delta 180.7,144.2,143.5,135.1,130.6,129.5,129.0(2 \mathrm{C}), 128.9$ (2C), $127.4,123.0,36.5$.

(E)-3-([1,1'-Biphenyl]-4-yl)-1-(1-methyl-1H-imidazol-2-yl)prop-2-en-1-one (1s)

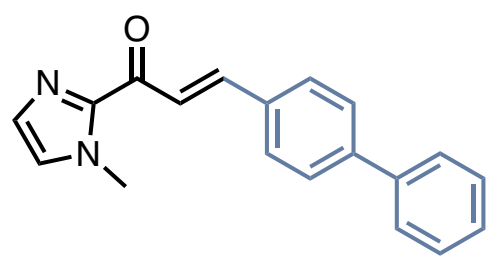

Molecular formula: $\mathrm{C}_{19} \mathrm{H}_{16} \mathrm{~N}_{2} \mathrm{O}$

$\mathrm{MW}=288.35 \mathrm{~g} \cdot \mathrm{mol}^{-1}$

The title compound was isolated as an off-white solid (590 mg, $2.06 \mathrm{mmol}$, 85\%) using general procedure E. Purified by flash column chromatography over silica gel (Toluene/Acetone $=95: 5$ ). Spectral data matched those reported in the literature. ${ }^{12}$

${ }^{1}$ H NMR (400 MHz, CDCl $) \delta 8.12(\mathrm{~d}, J=15.9 \mathrm{~Hz}, 1 \mathrm{H}), 7.87(\mathrm{~d}, J=16.0 \mathrm{~Hz}, 1 \mathrm{H}), 7.79-7.77$ $(\mathrm{m}, 2 \mathrm{H}), 7.66-7.62(\mathrm{~m}, 4 \mathrm{H}), 7.48-7.44(\mathrm{~m}, 2 \mathrm{H}), 7.38(\mathrm{td}, J=7.1,1.4 \mathrm{~Hz}, 1 \mathrm{H}), 7.09(\mathrm{~d}, J=0.9 \mathrm{~Hz}$, $1 \mathrm{H}), 7.09(\mathrm{~s}, 1 \mathrm{H}), 4.12(\mathrm{~s}, 3 \mathrm{H})$.

\footnotetext{
${ }^{12}$ Manguin, R.; Pichon, D.; Tarrieu, R.; Vives, T.; Roisnel, T.; Dorcet, V.; Crévisy, C.; Miqueu, K.; Favereau, L.; Crassous, J.; Mauduit, M.; Baslé, O. Chem. Commun. 2019, 55, 6058-6061.
} 
${ }^{13} \mathrm{C}$ NMR $\left(101 \mathrm{MHz}, \mathrm{CDCl}_{3}\right) \delta 180.5,144.1,143.2,143.0,140.2,133.9,129.3,129.3,128.9$, $127.8,127.5,127.3,127.1,122.7,36.4$.

(E)-1-(1-methyl-1H-imidazol-2-yl)-3-(naphthalen-2-yl)prop-2-en-1-one (1t)

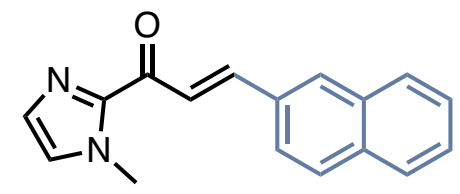

Molecular formula: $\mathrm{C}_{17} \mathrm{H}_{14} \mathrm{~N}_{2} \mathrm{O}$

$\mathrm{MW}=262.31 \mathrm{~g} \cdot \mathrm{mol}^{-1}$

The title compound was isolated as a beige solid (970 mg, $3.56 \mathrm{mmol}, 89 \%$ ) using general procedure E. Purified by flash column chromatography over silica gel (Toluene/Acetone $=95: 5$ ).

${ }^{1}$ H NMR $\left(400 \mathrm{MHz}, \mathrm{CDCl}_{3}\right) \delta 8.20(\mathrm{~d}, J=16.0 \mathrm{~Hz}, 1 \mathrm{H}), 8.09(\mathrm{~s}, 1 \mathrm{H}), 7.99(\mathrm{~d}, J=16.0 \mathrm{~Hz}, 1 \mathrm{H})$, 7.89-7.82 (m, 4H), 7.53-7.49 (m, 2H), $7.25(\mathrm{~s}, 1 \mathrm{H}), 7.09(\mathrm{~s}, 1 \mathrm{H}), 4.12(\mathrm{~s}, 3 \mathrm{H})$.

${ }^{13} \mathrm{C}$ NMR $\left(101 \mathrm{MHz}, \mathrm{CDCl}_{3}\right) \delta 180.5,143.4,134.4,133.4,132.5,130.6,129.4,128.7,128.6$, $127.3,126.6,124.3,123.0,36.4$.

HMRS: Calcd for [ $\left.\mathrm{C}_{17} \mathrm{H}_{15} \mathrm{~N}_{2} \mathrm{O}\right]+:$ 263.1184; Found: 263.1086.

IR $\left(\mathrm{cm}^{-1}\right):$ 3134, 3057, 2956, 1655, 1591, 1408, 1257, 1156, 1020, 979, 852, 754. 
${ }^{1} \mathrm{H}-\mathrm{NMR}$ of compound $1 \mathrm{t}\left(400 \mathrm{MHz}, \mathrm{CDCl}_{3}\right)$

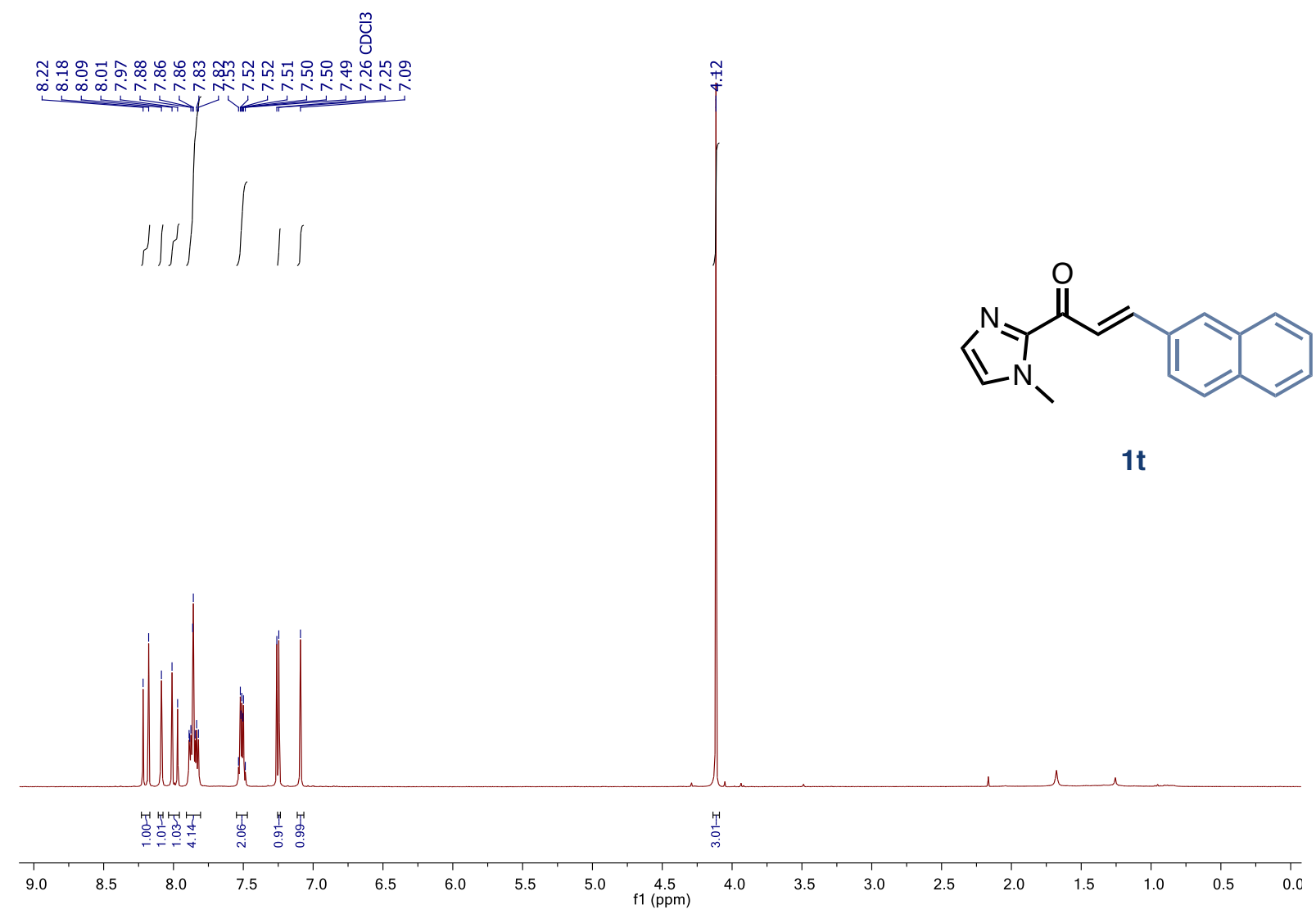

${ }^{13} \mathrm{C}-\mathrm{NMR}$ of compound 1t (101 $\left.\mathrm{MHz}, \mathrm{CDCl}_{3}\right)$

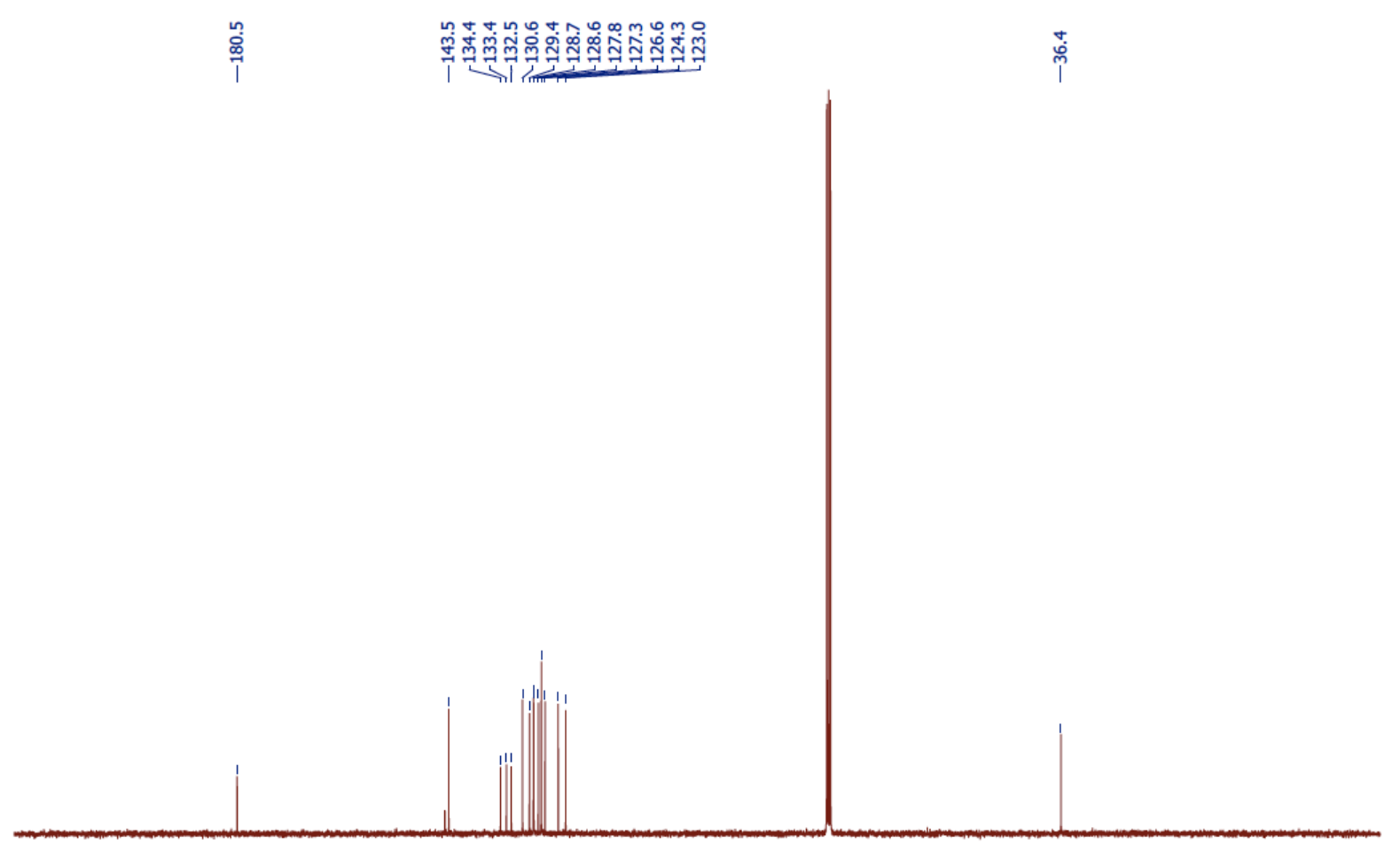

$\begin{array}{llllllllllllllllllllllllllllll}210 & 200 & 190 & 180 & 170 & 160 & 150 & 140 & 130 & 120 & 110 & 100 & 90 & 80 & 70 & 60 & 50 & 40 & 30 & 20 & 10 & 0 & -10 & \end{array}$ 


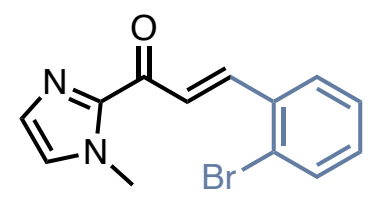

Molecular formula: $\mathrm{C}_{13} \mathrm{H}_{11} \mathrm{BrN}_{2} \mathrm{O}$

$\mathrm{MW}=291.15 \mathrm{~g} \cdot \mathrm{mol}^{-1}$

The title compound was isolated as a yellow solid (1.781 g, $6.12 \mathrm{mmol}, 75 \%)$ using general procedure E. Purified by flash column chromatography over silica gel $(P E / E t O A c=2: 1)$. Spectral data matched those reported in the literature. ${ }^{13}$

${ }^{1} \mathrm{H}$ NMR $\left(400 \mathrm{MHz}, \mathrm{CDCl}_{3}\right) \delta 8.21(\mathrm{~d}, J=15.9 \mathrm{~Hz}, 1 \mathrm{H}), 8.03(\mathrm{~d}, J=15.9 \mathrm{~Hz}, 1 \mathrm{H}), 7.85(\mathrm{~d}, J=7.9$, $1.7 \mathrm{~Hz}, 1 \mathrm{H}), 7.62(\mathrm{~d}, J=8.0,1.2 \mathrm{~Hz}, 1 \mathrm{H}), 7.33(\mathrm{~m}, 1 \mathrm{H}), 7.24-7.20(\mathrm{~m}, 2 \mathrm{H}), 7.09(\mathrm{~d}, J=0.4 \mathrm{~Hz}$, $1 \mathrm{H}), 4.10(\mathrm{~s}, 3 \mathrm{H})$.

${ }^{13} \mathrm{C}$ NMR $\left(101 \mathrm{MHz}, \mathrm{CDCl}_{3}\right) \delta 180.0,144.0,141.5,134.9,133.4,131.3,129.5,128.2,127.6$, $127.4,126.2,125.3,36.4$.

(E)-3-(3-Bromophenyl)-1-(1-methyl-1H-imidazol-2-yl)prop-2-en-1-one (1v)<smiles>Cn1ccnc1C(=O)/C=C/c1cccc(Br)c1</smiles>

Molecular formula: $\mathrm{C}_{13} \mathrm{H}_{11} \mathrm{BrN}_{2} \mathrm{O}$

$\mathbf{M W}=291.15 \mathrm{~g} \cdot \mathrm{mol}^{-1}$

The title compound was isolated as a beige solid (100 mg, $0.46 \mathrm{mmol}, 19 \%$ ) using general procedure E. Purified by flash column chromatography over silica gel (PE/EtOAC = 2:1). Spectral data matched those reported in the literature. ${ }^{14}$

${ }^{1} \mathbf{H}$ NMR $\left(400 \mathrm{MHz}, \mathrm{CDCl}_{3}\right) \delta 8.06(\mathrm{~d}, J=16.0 \mathrm{~Hz}, 1 \mathrm{H}), 7.85(\mathrm{t}, J=1.7 \mathrm{~Hz}, 1 \mathrm{H}), 7.72$ (d, $J=16.0 \mathrm{~Hz}, 1 \mathrm{H}$ ), 7.58 (d, $J=7.7 \mathrm{~Hz}, 1 \mathrm{H}), 7.51$ (ddd, $J=8.0,1.8,0.9 \mathrm{~Hz}, 1 \mathrm{H}), 7.29-7.23(\mathrm{~m}$, $2 \mathrm{H}), 7.23(\mathrm{~d}, J=0.8 \mathrm{~Hz}, 1 \mathrm{H}), 7.09(\mathrm{~s}, 1 \mathrm{H}), 4.09(\mathrm{~s}, 3 \mathrm{H})$.

\footnotetext{
${ }^{13}$ Benedetti, E.; Duchemin, N.; Bethge, L.; Vonhoff, S.; Klussmann, S.; Vasseur, J.-J.; Cossy, J.; Smietana, M.; Arseniyadis, S. Chem. Commun, 2015, 51, 6076-6079.

${ }^{14}$ Guan, X.-Y.; Yang, L.-P.; Hu, W. Angew. Chem., Int. Ed. 2010, 49, 2190-2192.
} 
${ }^{13} \mathrm{C}$ NMR $\left(101 \mathrm{MHz}, \mathrm{CDCl}_{3}\right) \delta 180.1,143.9,141.5,137.1,133.1,130.3,129.5,127.5,127.4$, 124.1, 123.01, 36.4.

(E)-3-(4-Bromophenyl)-1-(1-methyl-1H-imidazol-2-yl)prop-2-en-1-one (1w)<smiles>Cn1ccnc1C(=O)/C=C/c1ccc(Br)cc1</smiles>

Molecular formula: $\mathrm{C}_{13} \mathrm{H}_{11} \mathrm{BrN}_{2} \mathrm{O}$

$\mathbf{M W}=291.15 \mathrm{~g} \cdot \mathrm{mol}^{-1}$

The title compound was isolated as a pale yellow solid (534 mg, $1.83 \mathrm{mmol}, 76 \%$ ) using general procedure E. Purified by flash column chromatography over silica gel $(\mathrm{PE} / \mathrm{EtOAC}=2: 1)$. Spectral data matched those reported in the literature. ${ }^{15}$

${ }^{1}$ H NMR $\left(400 \mathrm{MHz}, \mathrm{CDCl}_{3}\right) \delta 8.06(\mathrm{~d}, J=16.0 \mathrm{~Hz}, 1 \mathrm{H}), 7.74(\mathrm{~d}, J=16.0 \mathrm{~Hz}, 1 \mathrm{H}), 7.57-7.52$ (m, 4H), $7.22(\mathrm{~d}, J=1.0 \mathrm{~Hz}, 1 \mathrm{H}), 7.09(\mathrm{~d}, J=0.9 \mathrm{~Hz}, 1 \mathrm{H}), 4.10(\mathrm{~s}, 3 \mathrm{H})$.

${ }^{13} \mathrm{C}$ NMR $\left(101 \mathrm{MHz}, \mathrm{CDCl}_{3}\right) \delta 180.2,141.9,133.9,132.1,130.1,129.4,127.4,124.7,123.4$, 36.4.

(E)-3-(4-Chlorophenyl)-1-(1-methyl-1H-imidazol-2-yl)prop-2-en-1-one (1x)<smiles>Cn1ccnc1C(=O)/C=C/c1ccc(Cl)cc1</smiles>

Molecular formula: $\mathrm{C}_{13} \mathrm{H}_{11} \mathrm{~N}_{2} \mathrm{O}$

$\mathrm{MW}=246.69 \mathrm{~g} \cdot \mathrm{mol}^{-1}$

The title compound was isolated as a yellow solid (466 mg, 70\% yield) using general procedure E. Purified by flash column chromatography over silica gel (Tol/Acetone = 95:5) . Spectral data matched those reported in the literature. ${ }^{1}$

${ }^{1}$ H NMR $\left(400 \mathrm{MHz} \mathrm{CDCl}_{3}\right) \delta 8.08(\mathrm{~d}, J=16.0 \mathrm{~Hz}, 1 \mathrm{H}), 7.77(\mathrm{~d}, J=16.0 \mathrm{~Hz}, 1 \mathrm{H})$, 7.67-7.60 (m, $2 \mathrm{H}), 7.41-7.35(\mathrm{~m}, 2 \mathrm{H}), 7.24(\mathrm{~s}, 1 \mathrm{H}), 7.10(\mathrm{~s}, 1 \mathrm{H}), 4.10(\mathrm{~s}, 3 \mathrm{H})$.

${ }^{15}$ Myers, M. C.; Bharadwadj, A. R.; Milgram, B. C.; Scheidt, K. A. J. Am. Chem. Soc. 2005, 127, 14675-14680. 
${ }^{13} \mathrm{C}$ NMR $\left(101 \mathrm{MHz}, \mathrm{CDCl}_{3}\right) \delta 179.8,143.5,142.1,136.3,133.3,129.8$ (2C), 129.0 (2C), 128.8, $127.2,123.1,36.3$.

(E)-3-(2,4-Dichlorophenyl)-1-(1-methyl-1H-imidazol-2-yl)prop-2-en-1-one (1y)<smiles>Cn1ccnc1C(=O)/C=C/c1ccc(Cl)cc1Cl</smiles>

Molecular formula: $\mathrm{C}_{13} \mathrm{H}_{10} \mathrm{Cl}_{2} \mathrm{~N}_{2} \mathrm{O}$

$\mathrm{MW}=281.14 \mathrm{~g} \cdot \mathrm{mol}^{-1}$

The title compound was isolated as an off-white solid (385 mg, $1.43 \mathrm{mmol}, 59 \%$ ) using general procedure E. Purified by flash column chromatography over silica gel (Toluene/Acetone $=95: 5$ ).

${ }^{1}$ H NMR $\left(400 \mathrm{MHz}, \mathrm{CDCl}_{3}\right) \delta 8.17(\mathrm{~d}, J=16.0 \mathrm{~Hz}, 1 \mathrm{H}), 8.04(\mathrm{~d}, J=16.0 \mathrm{~Hz}, 1 \mathrm{H}), 7.80$ (d, $J=8.5 \mathrm{~Hz}, 1 \mathrm{H}), 7.45(\mathrm{~d}, J=2.1 \mathrm{~Hz}, 1 \mathrm{H}), 7.28(\mathrm{~m}, 1 \mathrm{H}), 7.22(\mathrm{~d}, J=1.0 \mathrm{~Hz}, 1 \mathrm{H}), 7.10(\mathrm{~s}, 1 \mathrm{H}), 4.10$ $(\mathrm{s}, 3 \mathrm{H})$.

${ }^{13} \mathrm{C}$ NMR $\left(101 \mathrm{MHz}, \mathrm{CDCl}_{3}\right) \delta 179.9,144.0,137.7,136.5,136.3,131.9,130.1,129.7,128.8$, $127.7,127.6,125.5,36.5$.

HRMS (ESI) m/z: [M+H] $]^{+}$calcd for $\mathrm{C}_{13} \mathrm{H}_{11} \mathrm{Cl}_{2} \mathrm{~N}_{2} \mathrm{O}$ 281.0248; Found: 281.0244.

IR $\left(\mathrm{cm}^{-1}\right): 2978,2864,1662,1602,1582,1468,1409,1301,1054,1033,818$. 
${ }^{1} \mathrm{H}-\mathrm{NMR}$ of compound $1 \mathrm{y}\left(400 \mathrm{MHz}, \mathrm{CDCl}_{3}\right)$

范

$\underbrace{\infty}_{\infty}$

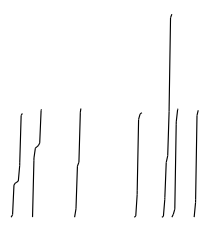

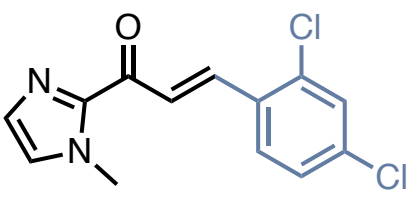

$1 y$

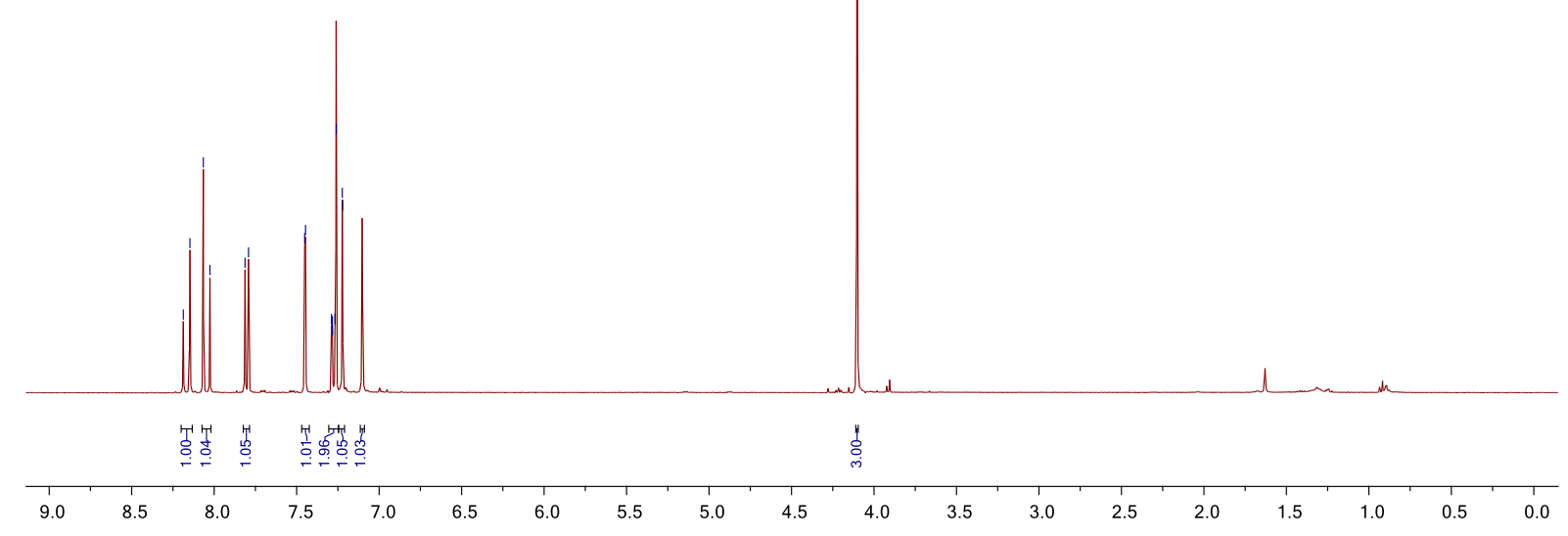

${ }^{13} \mathrm{C}-\mathrm{NMR}$ of compound 1y $\left(101 \mathrm{MHz}, \mathrm{CDCl}_{3}\right)$

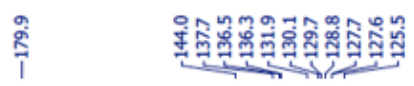

î 
(E)-1-(1-Methyl-1H-imidazol-2-yl)-3-(4-(trifluoromethyl)phenyl)prop-2-en-1-one (1z)

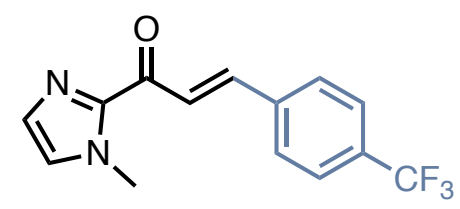

Molecular formula: $\mathrm{C}_{14} \mathrm{H}_{11} \mathrm{~F}_{3} \mathrm{~N}_{3} \mathrm{O}$

$\mathrm{MW}=280.25 \mathrm{~g} \cdot \mathrm{mol}^{-1}$

The title compound was isolated as a white solid (423 mg, $1.51 \mathrm{mmol}, 47 \%$ ) using general procedure E. Purified by flash column chromatography over silica gel (PE/Acetone $=4: 1$ ). Spectral data matched those reported in the literature. ${ }^{16}$

${ }^{1}$ H NMR $\left(400 \mathrm{MHz}^{\mathrm{C}} \mathrm{CDCl}_{3}\right) \delta 8.14(\mathrm{~d}, J=16.1 \mathrm{~Hz}, 1 \mathrm{H}), 7.84-7.75(\mathrm{~m}, 3 \mathrm{H}), 7.65(\mathrm{~d}, J=8.2 \mathrm{~Hz}$, $2 \mathrm{H}), 7.23(\mathrm{~s}, 1 \mathrm{H}), 7.10(\mathrm{~s}, 1 \mathrm{H}), 4.10(\mathrm{~s}, 3 \mathrm{H})$.

${ }^{13} \mathrm{C}$ NMR $\left(101 \mathrm{MHz}, \mathrm{CDCl}_{3}\right) \delta 180.1,144.0,141.3,138.4,131.9$ (q, $\left.J=32.4 \mathrm{~Hz}\right), 129.7,128.9$, 127.7, 125.9 (d, J = 3.8 Hz), 125.3, 124.0 (q, J = 272.0 Hz, CF3), 36.5.

${ }^{19}$ F NMR $\left(377 \mathrm{MHz}, \mathrm{CDCl}_{3}\right) \delta-62.83$.

(E)-1-(1-Methyl-1H-imidazol-2-yl)-3-(4-nitrophenyl)prop-2-en-1-one (1aa)

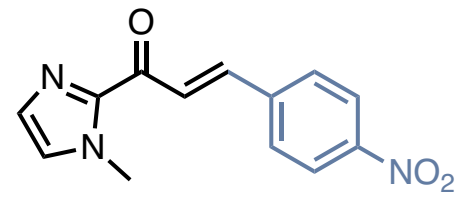

Molecular formula: $\mathrm{C}_{13} \mathrm{H}_{11} \mathrm{~N}_{3} \mathrm{O}_{3}$

$\mathrm{MW}=257.25 \mathrm{~g} \cdot \mathrm{mol}^{-1}$

The title compound was isolated as an off-white solid (450 mg, $1.75 \mathrm{mmol}, 72 \%$ ) using general procedure E. Purified by flash column chromatography over silica gel (Toluene/Acetone = 95:5).

${ }^{1}$ H NMR $\left(400 \mathrm{MHz}, \mathrm{CDCl}_{3}\right) \delta 8.29-8.23(\mathrm{~m}, 2 \mathrm{H}), 8.19(\mathrm{~d}, J=16.0 \mathrm{~Hz}, 1 \mathrm{H}), 7.85-7.78(\mathrm{~m}, 3 \mathrm{H})$, $7.25(\mathrm{~d}, J=0.9 \mathrm{~Hz}, 1 \mathrm{H}), 7.13(\mathrm{~s}, 1 \mathrm{H}), 4.11(\mathrm{~s}, 3 \mathrm{H})$.

${ }^{13} \mathrm{C}$ NMR $\left(101 \mathrm{MHz}, \mathrm{CDCl}_{3}\right) \delta 179.7,148.6,143.9,141.3,140.1,129.9,129.3,127.9,126.9$, 124.3, 36.5.

HRMS (ESI) m/z: [M+H] calcd for $\mathrm{C}_{13} \mathrm{H}_{12} \mathrm{~N}_{3} \mathrm{O}_{3}$ 258.0879; Found: 258.0870.

IR $\left(\mathrm{cm}^{-1}\right):$ 2973, 1655, 1609, 1592, 1511, 1399, 1344, 1054, 1033, 1015, 841, 797, 778, 734.

${ }^{16}$ Albaladejo, M. J.; Alonson, F.; Gonzalez-Soria, M. J. ACS. Catal. 2015, 5, 3446-3456. 

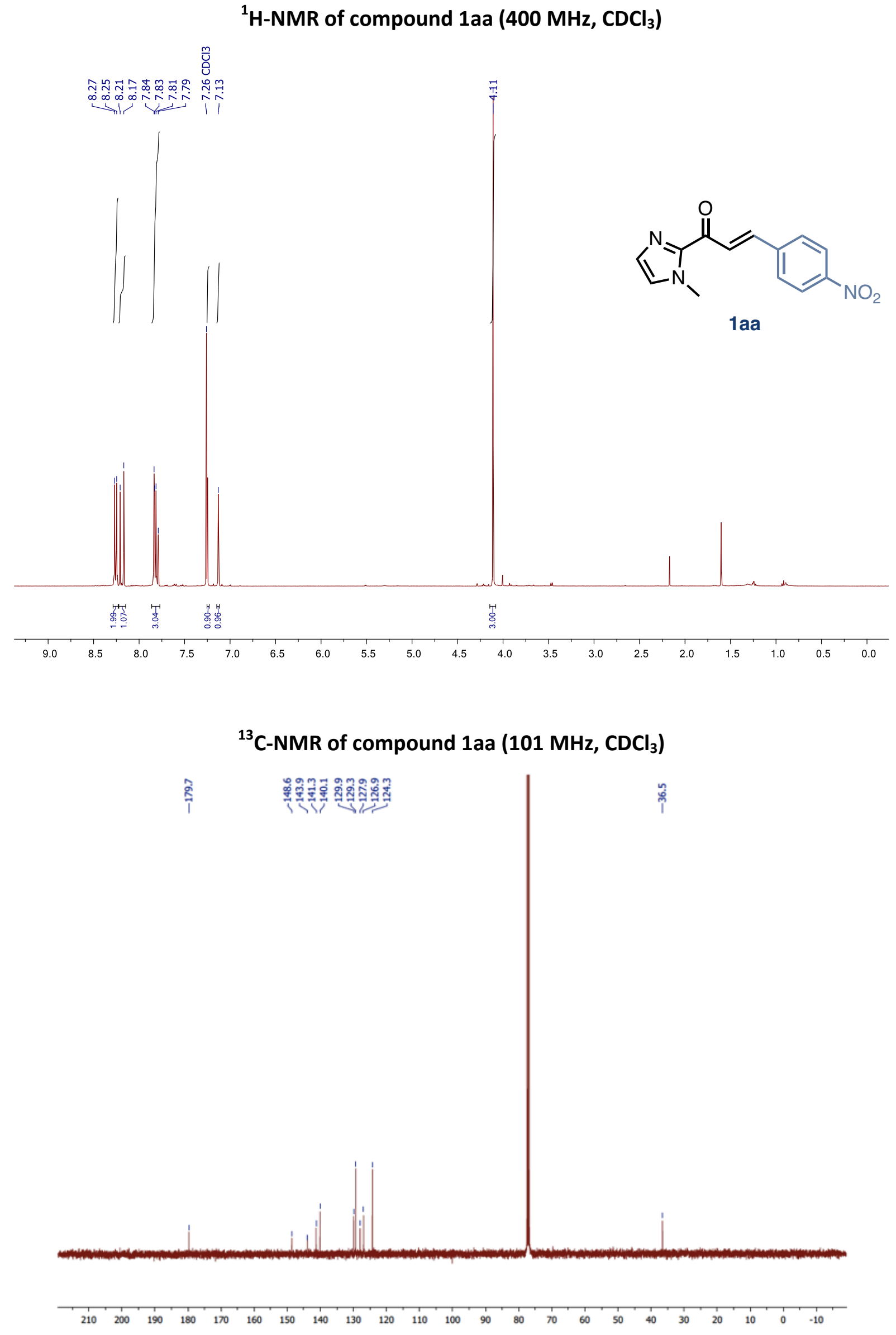


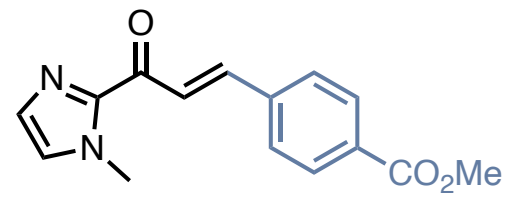

Molecular formula: $\mathrm{C}_{15} \mathrm{H}_{14} \mathrm{~N}_{2} \mathrm{O}_{3}$

$\mathrm{MW}=270.29 \mathrm{~g} \cdot \mathrm{mol}^{-1}$

The title compound was isolated as an off-white solid (385 mg, $1.43 \mathrm{mmol}, 59 \%$ ) using general procedure E. Purified by flash column chromatography over silica gel $(\mathrm{PE} / \mathrm{EtOAC}=2: 1)$. Spectral data matched those reported in the literature. ${ }^{17}$

${ }^{1}$ H NMR $\left(400 \mathrm{MHz} \mathrm{CDCl}_{3}\right) \delta 8.15(\mathrm{~d}, J=16.1 \mathrm{~Hz}, 1 \mathrm{H}), 8.08-8.05(\mathrm{~m}, 2 \mathrm{H}), 7.82(\mathrm{~d}, J=16.1 \mathrm{~Hz}$, 1H), 7.76-7.73 (m, 2H), $7.24(\mathrm{~d}, J=0.9 \mathrm{~Hz}, 1 \mathrm{H}), 7.10(\mathrm{~s}, 1 \mathrm{H}), 4.11(\mathrm{~s}, 3 \mathrm{H}), 3.94(\mathrm{~s}, 3 \mathrm{H})$.

${ }^{13} \mathrm{C}$ NMR $\left(101 \mathrm{MHz}, \mathrm{CDCl}_{3}\right) \delta 180.1,166.5,143.9,141.7,139.2,131.4,130.1,129.6,128.5$, $127.5,125.1,52.3,36.4$.

(E)-3-(4-Methoxyphenyl)-1-(1-methyl-1H-imidazol-2-yl)prop-2-en-1-one (1ac)<smiles>COc1ccc(/C=C/C(=O)c2nccn2C)cc1</smiles>

Molecular formula: $\mathrm{C}_{14} \mathrm{H}_{14} \mathrm{~N}_{2} \mathrm{O}_{2}$

$\mathrm{MW}=242.28 \mathrm{~g} \cdot \mathrm{mol}^{-1}$

The title compound was isolated as a yellow solid (459 $\mathrm{mg} 81 \%$ yield) using general procedure E. Purified by flash column chromatography over silica gel (PE/EtOAc = 2:1). Spectral data matched those reported in the literature. ${ }^{1}$

${ }^{1}$ H NMR $\left(400 \mathrm{MHz}, \mathrm{CDCl}_{3}\right) \delta 7.94(\mathrm{~d}, J=15.9 \mathrm{~Hz}, 1 \mathrm{H}), 7.75(\mathrm{~d}, J=15.9 \mathrm{~Hz}, 1 \mathrm{H}), 7.65-7.56$ $(\mathrm{m}, 2 \mathrm{H}), 7.17(\mathrm{~d}, J=0.9 \mathrm{~Hz}, 1 \mathrm{H}), 7.01(\mathrm{~d}, J=0.9 \mathrm{~Hz}, 1 \mathrm{H}), 6.89-6.83(\mathrm{~m}, 2 \mathrm{H}), 4.04(\mathrm{~s}, 3 \mathrm{H}), 3.78$ $(\mathrm{s}, 3 \mathrm{H})$. 17 Jacob, N. T.; Miranda, P. O.; Shirey, R. J.; Gautam, R.; Zhin, B.; de Orbe Izquierdo, M. E.; Hixon, M. S.; Ueno,
L.; Vogt, P. K.; Janda, K. D. Biorg. Med. Chem. 2019, 26, 4234-4239. 
${ }^{13} \mathrm{C}$ NMR $\left(101 \mathrm{MHz}, \mathrm{CDCl}_{3}\right) \delta 180.8,161.8,144.3,143.4,130.7$ (2C), 129.3, 127.9, 127.2, $120.7,114.5(2 C), 55.5,36.5$.

(E)-3-(furan-2-yl)-1-(1-methyl-1H-imidazol-2-yl)prop-2-en-1-one (1ad)<smiles>Cn1ccnc1C(=O)/C=C/c1ccco1</smiles>

Molecular formula: $\mathrm{C}_{11} \mathrm{H}_{10} \mathrm{~N}_{2} \mathrm{O}_{2}$

$\mathrm{MW}=202.21 \mathrm{~g} \cdot \mathrm{mol}^{-1}$

The title compound was isolated as a yellow solid (1.27 g, $6.24 \mathrm{mmol}, 77 \%)$ using general procedure E. Purified by flash column chromatography over silica gel $\left(\mathrm{PE} / \mathrm{Et}_{2} \mathrm{O}=2: 1\right)$. Spectral data matched those reported in the literature. ${ }^{14}$

${ }^{1}$ H NMR $\left(400 \mathrm{MHz} \mathrm{CDCl}_{3}\right) \delta 7.91(\mathrm{~d}, J=15.8 \mathrm{~Hz}, 1 \mathrm{H}), 7.58(\mathrm{~d}, J=15.8 \mathrm{~Hz}, 1 \mathrm{H}), 7.51(\mathrm{~m}, 1 \mathrm{H})$, $7.20(\mathrm{~d}, J=0.9 \mathrm{~Hz}, 1 \mathrm{H}), 7.05(\mathrm{~d}, J=0.4 \mathrm{~Hz}, 1 \mathrm{H}), 6.73(\mathrm{~m}, 1 \mathrm{H}), 6.48(\mathrm{~d}, J=0.4 \mathrm{~Hz}, 1 \mathrm{H}), 4.07$ $(\mathrm{s}, 3 \mathrm{H})$.

${ }^{13} \mathrm{C}$ NMR $\left(101 \mathrm{MHz}, \mathrm{CDCl}_{3}\right) \delta 180.4,152.0,145.0,144.1,129.5,129.4,127.1,120.9,115.6$, $112.5,36.3$.

(E)-1-(1-methyl-1H-imidazol-2-yl)-3-(thiophen-2-yl)prop-2-en-1-one (1ae)<smiles>Cn1ccnc1C(=O)/C=C/c1cccs1</smiles>

Molecular formula: $\mathrm{C}_{11} \mathrm{H}_{10} \mathrm{~N}_{2} \mathrm{OS}$

$\mathbf{M W}=218 \cdot 27 \mathrm{~g} \cdot \mathrm{mol}^{-1}$

The title compound was isolated as a beige solid (530 mg, $2.43 \mathrm{mmol}, 81 \%$ ) using general procedure E. Purified by flash column chromatography over silica gel $\left(\mathrm{PE} / \mathrm{Et}_{2} \mathrm{O}=2: 1\right)$. Spectral data matched those reported in the literature. ${ }^{18}$

${ }^{18}$ Myers, M. C.; Bharadwaj, A. R.; Milgram, B. C.; Scheidt, K. A. J. Am. Chem. Soc., 2005, 127, 14675-14680. 
${ }^{1} \mathbf{H}$ NMR $\left(400 \mathrm{MHz}, \mathrm{CDCl}_{3}\right) \delta 7.96(\mathrm{~d}, J=15.6 \mathrm{~Hz}, 1 \mathrm{H}), 7.87(\mathrm{~d}, J=15.7 \mathrm{~Hz}, 1 \mathrm{H}), 7.43$ (dt, $J=5.1,1.0 \mathrm{~Hz}, 1 \mathrm{H}), 7.40(\mathrm{dd}, J=3.7,1.0 \mathrm{~Hz}, 1 \mathrm{H}), 7.23(\mathrm{~d}, J=1.0 \mathrm{~Hz}, 1 \mathrm{H}), 7.09-7.07$ (m, $2 \mathrm{H}), 4.09(\mathrm{~s}, 3 \mathrm{H})$.

${ }^{13} \mathrm{C}$ NMR $\left(101 \mathrm{MHz}, \mathrm{CDCl}_{3}\right) \delta 180.1,143.8,140.7,136.2,132.1,129.4,129.1,128.4,127.3$, 121.8, 36.6.

(2E,4E)-1-(1-Methyl-1H-imidazol-2-yl)hexa-2,4-dien-1-one (1af)

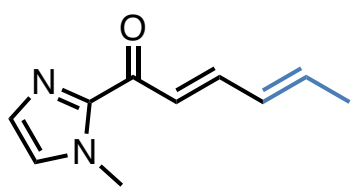

Molecular formula: $\mathrm{C}_{10} \mathrm{H}_{12} \mathrm{~N}_{2} \mathrm{O}$

$\mathrm{MW}=176.22 \mathrm{~g} \cdot \mathrm{mol}^{-1}$

The title compound was isolated as an off-white solid (385 mg, $1.43 \mathrm{mmol}, 59 \%$ ) using general procedure E. Purified by flash column chromatography over silica gel $(P E / E t O A C=3: 1)$. Spectral data matched those reported in the literature. ${ }^{15}$

${ }^{1} \mathbf{H}$ NMR $\left(400 \mathrm{MHz}, \mathrm{CDCl}_{3}\right) \delta$ 7.49-7.34 (m, 2H), $7.17(\mathrm{~d}, J=0.9 \mathrm{~Hz}, 1 \mathrm{H}), 7.03(\mathrm{~d}, J=0.9 \mathrm{~Hz}$, 1H), 6.40-6.20 (m, 2H), $4.05(\mathrm{~s}, 3 \mathrm{H}), 1.89(\mathrm{~d}, J=5.8 \mathrm{~Hz}, 3 \mathrm{H})$.

${ }^{13} \mathrm{C}$ NMR $\left(101 \mathrm{MHz}^{\mathrm{CDCl}} \mathrm{CDC}_{3} \delta 181.2,144.2,144.1,141.2,130.9,129.1,127.1,124.3,36.5\right.$, 19.1. 


\section{General Procedures: Alkenyl nitriles synthesis}

General procedure for the preparation of racemic Michael addition/retro-Dieckmann products
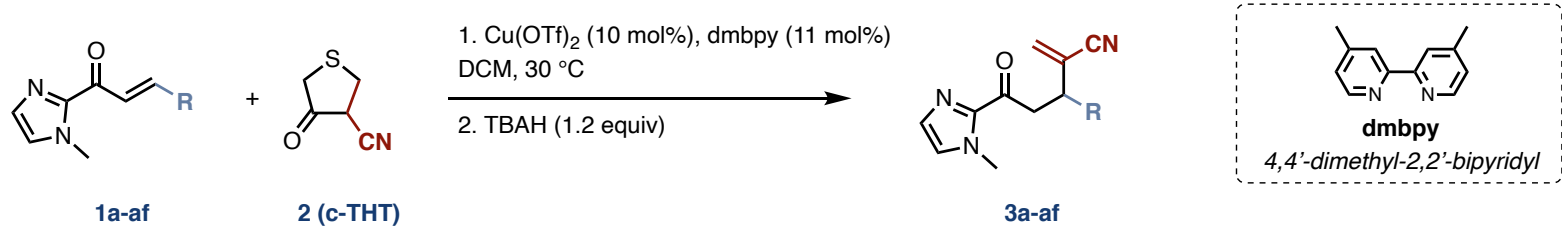

$\mathrm{Cu}(\mathrm{OTf})_{2}$ (10 mol\%) and 4,4-dimethyl-2,2'-bipyridyl (dmbpy) (11 mol\%) were added to the reaction mixture were dissolved in DCM. The desired enone 1a-af (1.0 equiv.) was dissolved in DCM (to reach a final concentration of $0.1 \mathrm{M}$ ), followed by c-THT (4-cyano-3-oxotetrahydrothiophene) (1.1 equiv.) and the resulting reaction mixture was stirred at $30{ }^{\circ} \mathrm{C}$ using a heating mantle. Upon completion, tetrabutylammonium hydroxide (TBAH) (1M in MeOH) (1.2 equiv.) was added and the reaction mixture was stirred for 2 min. The reaction mixture was then diluted with a saturated aqueous solution of $\mathrm{NaHCO}_{3}(5 \mathrm{~mL})$ and the aqueous phase was extracted with DCM $(3 \times 10 \mathrm{~mL})$. The combined organic layers were dried over anhydrous $\mathrm{MgSO}_{4}$ and concentrated. The crude residue was purified by flash column chromatography over silica gel using either $\mathrm{Hex} / \mathrm{Et}_{2} \mathrm{O}$ or $\mathrm{Hex} / \mathrm{EtOAc}$ or Hex/EtOAc/Acetone.

\section{General procedure for the optimization study}
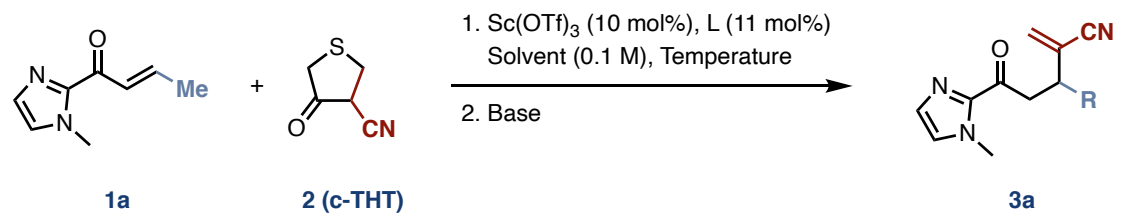

An oven-dried reaction vial was charged with the desired Lewis acid ( $0.02 \mathrm{mmol}, 0.1$ equiv.) and chiral ligand (0.022 mmol, 0.11 equiv.) then dried for $1 \mathrm{~h}$ under high-vacuum. Solvent $(1.5 \mathrm{~mL})$ was added and catalyst formation was ensured by stirring the resulting solution for $1 \mathrm{~h}$ at rt. Enone 1a (30 mg, $0.2 \mathrm{mmol}, 1.0$ equiv.) was also dried under high-vacuum for $15 \mathrm{~min}$, dissolved in solvent $(0.5 \mathrm{~mL})$. The resulting solution was cooled to the chosen temperature, and 4-cyano-3-oxotetrahydrothiophene (c-THT) (25 mg, $0.22 \mathrm{mmol}$, 1.1 equiv.) was added. Upon completion of the reaction as indicated by TLC 
(toluene/acetone 9:1), the chosen base (1.2 - 2.1 equiv.) was added and the reaction mixture was stirred until completion, as indicated by TLC (Toluene/Acetone 9:1, ninhydrin). The reaction mixture was then diluted with a saturated aqueous solution of $\mathrm{NaHCO}_{3}(5 \mathrm{~mL})$ and the aqueous phase was extracted with $\mathrm{DCM}(3 \times 10 \mathrm{~mL})$. The combined organic layers were dried over anhydrous $\mathrm{MgSO}_{4}$ and concentrated. The crude residue was analysed by NMR and chiral HPLC. The yield was determined by ${ }^{1} \mathrm{H} N M R$ using $\mathrm{CH}_{2} \mathrm{Br}_{2}$ as internal standard. The enantiomeric excess (ee) was determined by HPLC analysis of the crude reaction mixture.

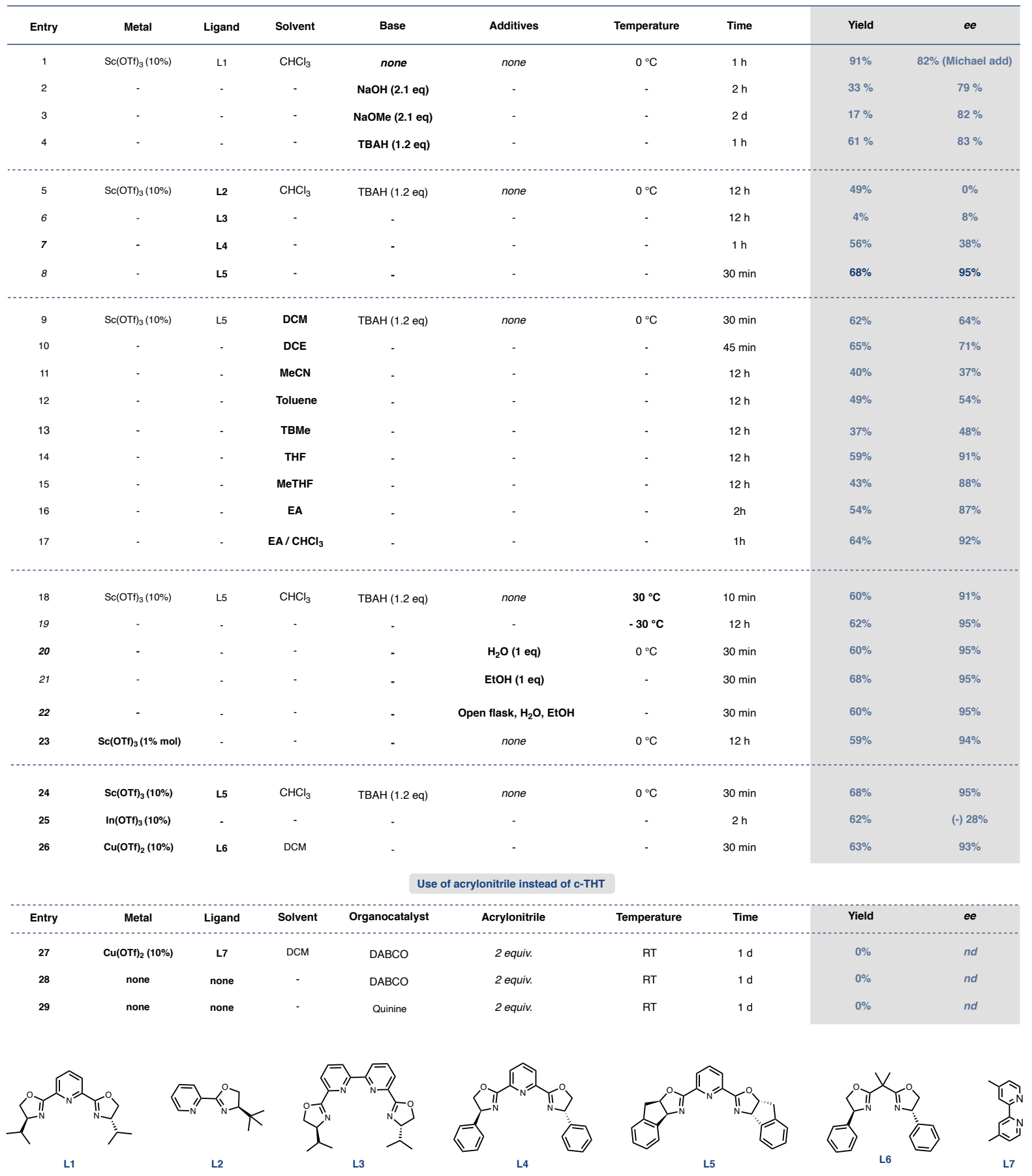




\section{Mechanistic hypothesis}

\section{Retro-Michael vs Retro-Dieckmann}

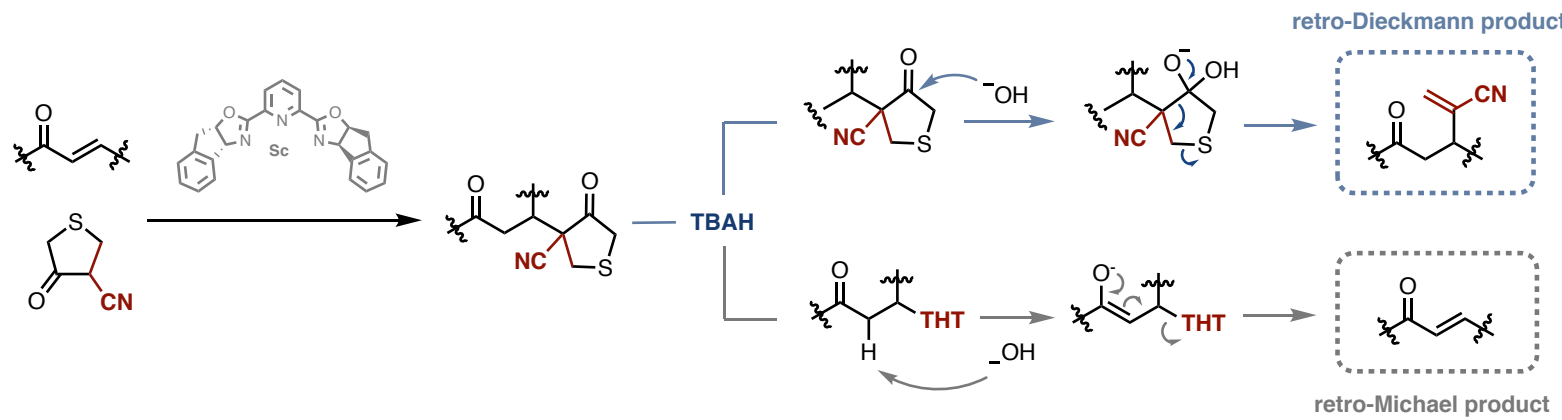

Figure S1: Mechanistic hypothesis for the by-product formation

\section{Investigation on the enantioselectivity outcome}
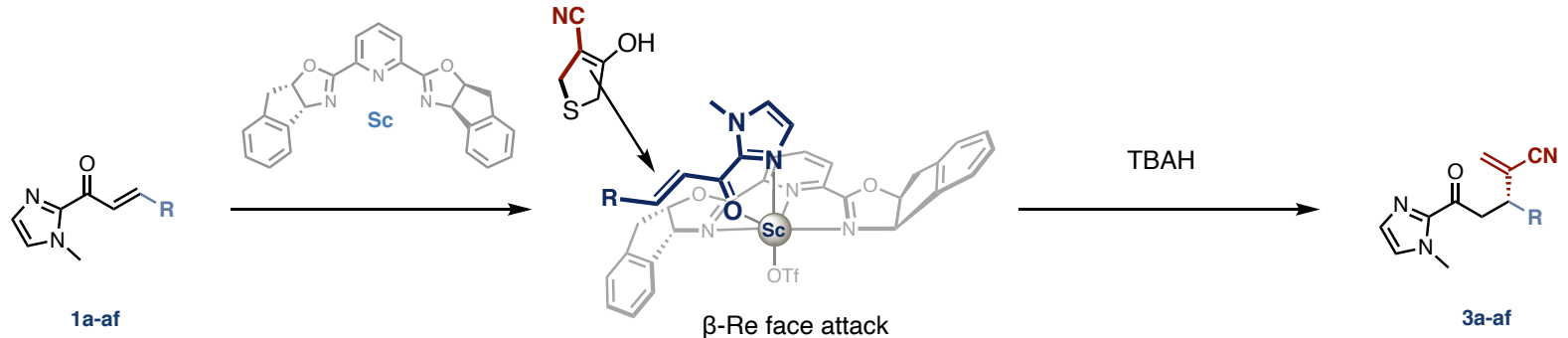

Figure S2: Mechanistic hypothesis for the enantioselectivity outcome 
General procedure $\mathrm{F}$ for the asymmetric Sc-catalyzed sequence
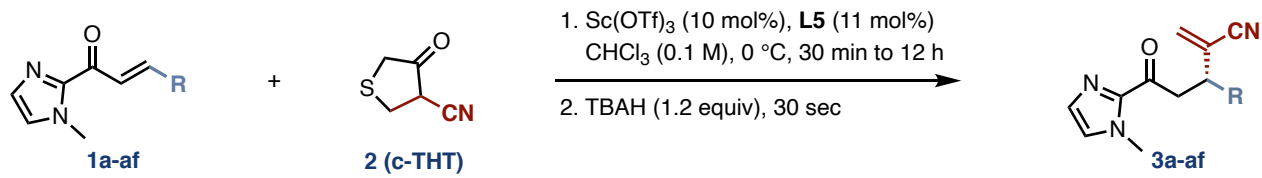

An oven-dried reaction vial was charged with $\mathrm{Sc}(\mathrm{OTf})_{3}$ (Alfa Aesar) (13 mg, $0.027 \mathrm{mmol}$, 0.1 equiv.) and 2,6-bis[(3aR,8aS)-(+)-8H-indeno[1,2- $d]$ oxazolin-2-yl)pyridine L5 (SigmaAldrich) (12 mg, $0.03 \mathrm{mmol}, 0.11$ equiv.) then dried for $1 \mathrm{~h}$ under high-vacuum. Dry $\mathrm{CHCl}_{3}$ $(2 \mathrm{~mL})$ was added, and catalyst formation was ensured by stirring the resulting solution for $1 \mathrm{~h}$ at rt. Enone 1a-af ( $0.27 \mathrm{mmol}, 1.0$ equiv.) was also dried under high-vacuum for $15 \mathrm{~min}$, dissolved in dry $\mathrm{CHCl}_{3}(0.7 \mathrm{~mL})$. The resulting solution was cooled to $0{ }^{\circ} \mathrm{C}$, and $\mathrm{c}$-THT $(38 \mathrm{mg}$, $0.3 \mathrm{mmol}, 1.1$ equiv.) was added. Upon completion of the reaction as indicated by TLC (Toluene/Acetone $=9: 1$ ), or if the reaction was not complete, after $2 \mathrm{~d}$, TBAH (1.2 equiv.) was added and the reaction mixture was stirred for $2 \mathrm{~min}$. The reaction mixture was then diluted with a saturated aqueous solution of $\mathrm{NaHCO}_{3}(5 \mathrm{~mL})$ and the aqueous phase was extracted with DCM (3 $\times 10 \mathrm{~mL})$. The combined organic layers were dried over anhydrous $\mathrm{MgSO}_{4}$ and concentrated. The crude residue was purified by flash column chromatography over silica gel using either $\mathrm{Hex} / \mathrm{Et}_{2} \mathrm{O}$, $\mathrm{Hex} / \mathrm{EtOAc}$ or Hex/EtOAc/Acetone. 
3-Methyl-5-(1-methyl-1H-imidazol-2-yl)-2-methylene-5-oxopentanenitrile (3a)<smiles>C=C(C#N)[C@H](C)CC(=O)c1nccn1C</smiles>

Molecular formula: $\mathrm{C}_{11} \mathrm{H}_{13} \mathrm{~N}_{3} \mathrm{O}$

$\mathrm{MW}=203.25 \mathrm{~g} \cdot \mathrm{mol}^{-1}$

The title compound was isolated as a yellow oil $(2 \mathrm{mmol}$ scale: $264 \mathrm{mg}, 1.3 \mathrm{mmol}, 65 \%, 94 \%$ ee, $0.27 \mathrm{mmol}$ scale: $36 \mathrm{mg}, 0.17 \mathrm{mmol}, 62 \%, 95 \%$ ee) using general procedure F. Purified by flash column chromatography over silica gel (Hex/EtOAc/Acetone $=6: 3: 1$ ).

${ }^{1} \mathbf{H}$ NMR (400 MHz, CDCl $) \delta 7.13(\mathrm{~s}, 1 \mathrm{H}), 7.03(\mathrm{~s}, 1 \mathrm{H}), 5.83(\mathrm{~s}, 1 \mathrm{H}), 5.81(\mathrm{~s}, 1 \mathrm{H}), 3.98(\mathrm{~s}, 3 \mathrm{H})$, $3.37(\mathrm{dd}, J=16.9,7.4 \mathrm{~Hz}, 1 \mathrm{H}), 3.26(\mathrm{dd}, J=16.9,6.7 \mathrm{~Hz}, 1 \mathrm{H}), 3.13(\mathrm{dqd}, J=7.4,6.8,6.7 \mathrm{~Hz}$, $1 \mathrm{H}), 1.24(\mathrm{~d}, J=6.8 \mathrm{~Hz}, 3 \mathrm{H})$.

${ }^{13} \mathrm{C}$ NMR $\left(101 \mathrm{MHz} \mathrm{CDCl}_{3}\right) \delta 190.1,142.9,129.5,129.4,127.9,127.4,117.8,43.9,36.3,34.7$, 19.5.

HRMS (ESI) m/z: [M+H] ${ }^{+}$calcd for $\mathrm{C}_{11} \mathrm{H}_{14} \mathrm{~N}_{3} \mathrm{O}$ : 204.1137; found: 204.1049 .

IR $\left(\mathrm{cm}^{-1}\right):$ 3112, 2929, 2935, 2224, 1674, 1460, 1406, 1294.

$[\boldsymbol{a}]_{D}^{20}=-20.5\left(c\right.$ 1.20, $\left.\mathrm{CH}_{2} \mathrm{Cl}_{2}\right)$

HPLC analysis: carried out using a mixture of hexane and isopropyl alcohol (90:10 isocratic), at $1 \mathrm{~mL} \cdot \mathrm{min}^{-1}$, at $25^{\circ} \mathrm{C}$ on an IC column, with UV detection $(\lambda=280 \mathrm{~nm})$. Retention times: 21.5 and $24.1 \mathrm{~min}$. 
${ }^{1} \mathrm{H}-\mathrm{NMR}$ of compound $3 \mathrm{a}\left(400 \mathrm{MHz}, \mathrm{CDCl}_{3}\right)$

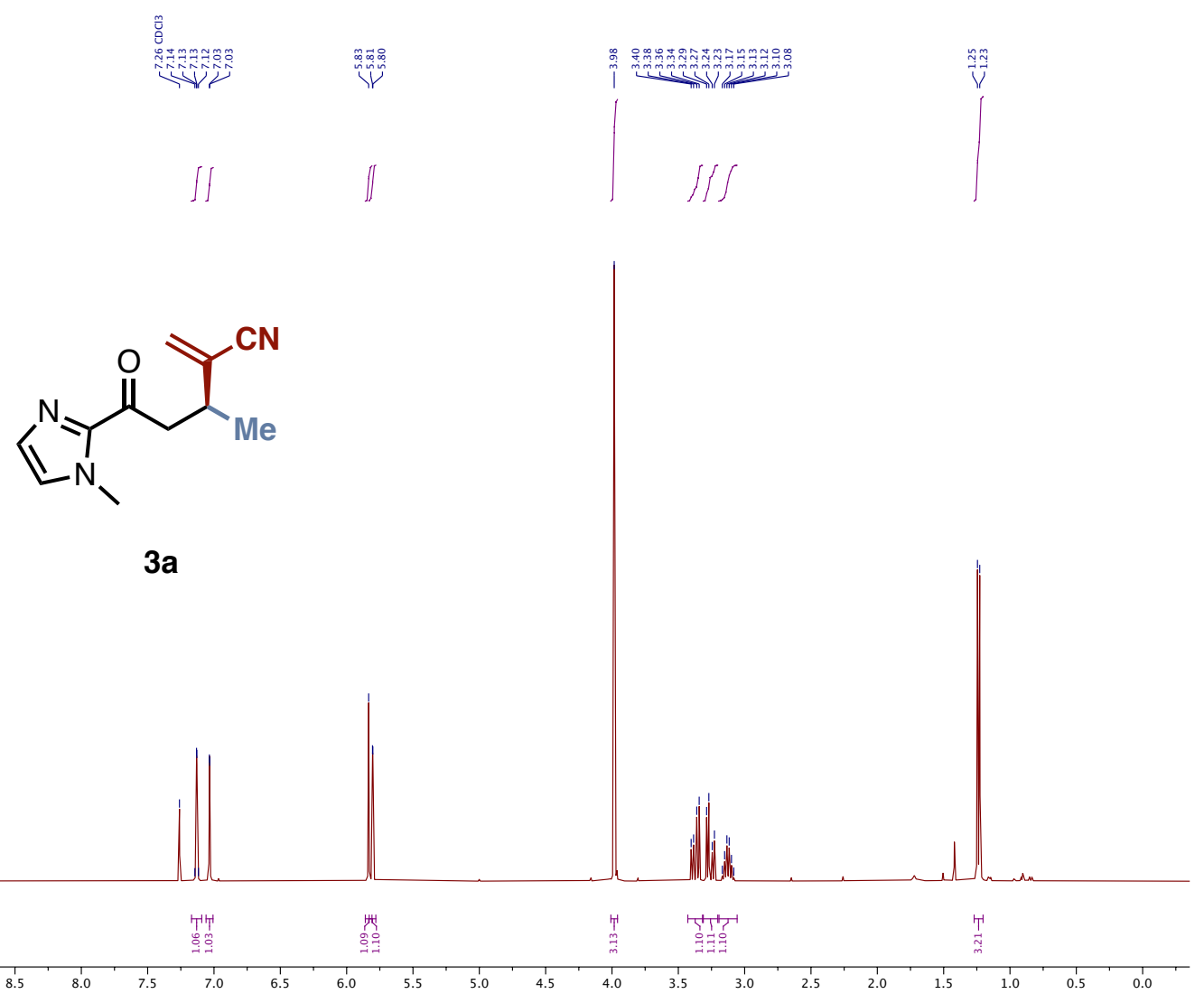

${ }^{13} \mathrm{C}-\mathrm{NMR}$ of compound 3a (101 $\left.\mathrm{MHz}, \mathrm{CDCl}_{3}\right)$

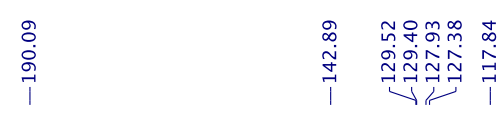

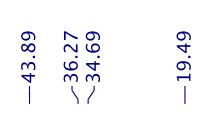



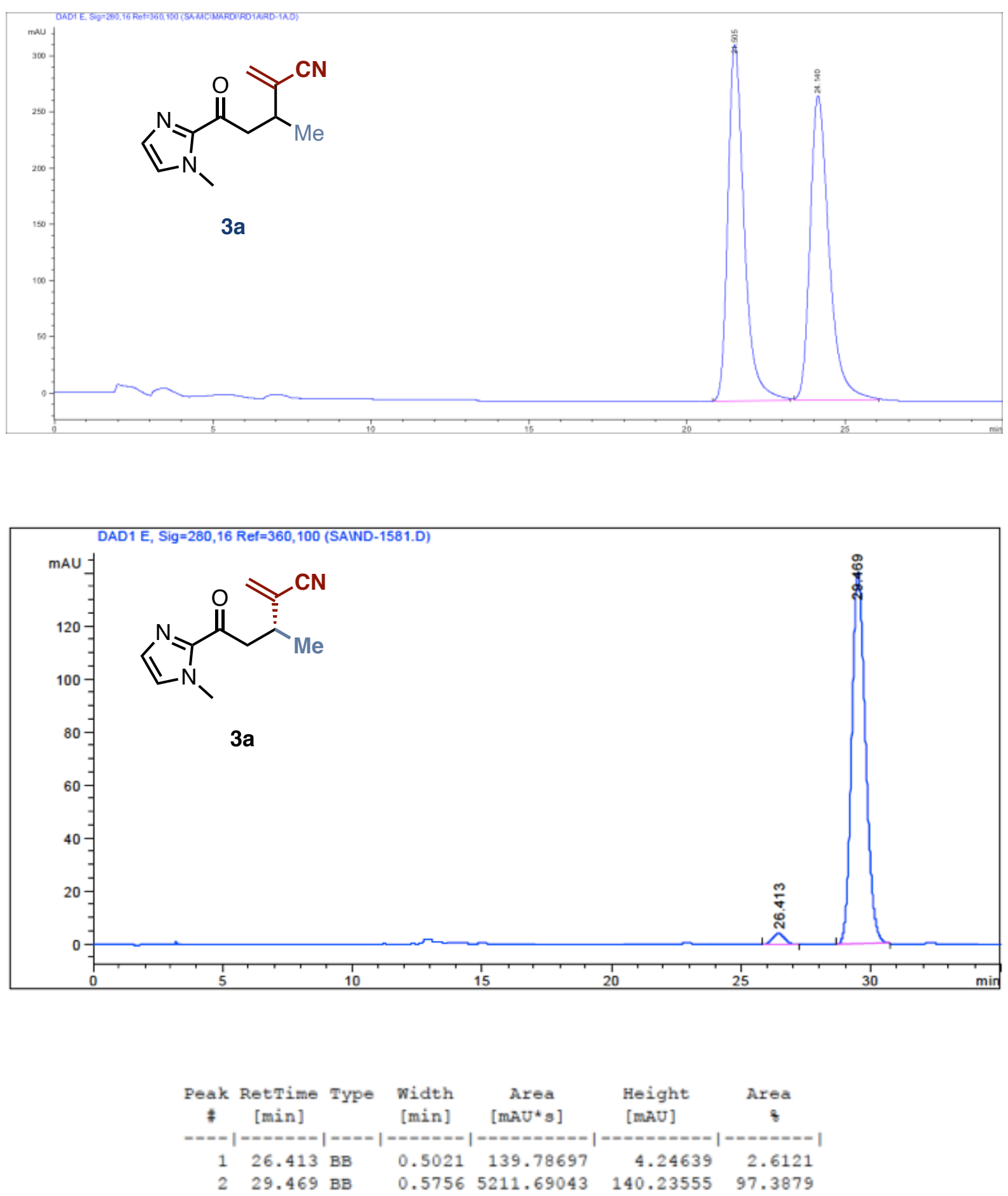


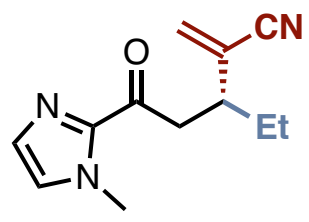

Molecular formula: $\mathrm{C}_{12} \mathrm{H}_{15} \mathrm{~N}_{3} \mathrm{O}$

$\mathbf{M W}=217.27 \mathrm{~g} \cdot \mathrm{mol}^{-1}$

The title compound was isolated as an amorphous solid (35 mg, $0.162 \mathrm{mmol}, 60 \%, 94 \%$ ee) using general procedure F. Purified by flash column chromatography over silica gel $($ Hex/EtOAc/Acetone $=6: 3: 1)$.

${ }^{1}$ H NMR $\left(400 \mathrm{MHz}, \mathrm{CDCl}_{3}\right) \delta 7.13(\mathrm{~d}, J=0.9 \mathrm{~Hz}, 1 \mathrm{H}), 7.03(\mathrm{~d}, J=0.9 \mathrm{~Hz}, 1 \mathrm{H}), 5.88(\mathrm{~s}, 1 \mathrm{H}), 5.79$ (s, $1 \mathrm{H}), 3.98(\mathrm{~s}, 3 \mathrm{H}), 3.38$ (dd, $J=17.1,8.4 \mathrm{~Hz}, 1 \mathrm{H}), 3.29(\mathrm{dd}, J=17.1,5.7 \mathrm{~Hz}, 1 \mathrm{H}), 2.90(\mathrm{~m}$, $1 \mathrm{H}), 1.67-1.56(\mathrm{~m}, 2 \mathrm{H}), 0.92(\mathrm{t}, J=7.4 \mathrm{~Hz}, 3 \mathrm{H})$.

${ }^{13} \mathrm{C}$ NMR $\left(101 \mathrm{MHz}, \mathrm{CDCl}_{3}\right) \delta 190.3,142.9,131.2,129.4,127.3,126.3,117.6,42.5,42.0,36.3$, $26.5,11.5$.

HRMS (ESI) m/z: [M+H] $]^{+}$calcd for $\mathrm{C}_{12} \mathrm{H}_{16} \mathrm{~N}_{3} \mathrm{O}$ 218.1293; found: 218.1267.

IR $\left(\mathrm{cm}^{-1}\right):$ 3112, 2929, 2935, 2224, 1674, 1460, 1406, 1294.

$[\boldsymbol{a}]_{D}^{20}=-32.3\left(c 0.87, \mathrm{CH}_{2} \mathrm{Cl}_{2}\right)$

HPLC analysis: carried out using a mixture of hexane and isopropyl alcohol (80:20 isocratic) at $1 \mathrm{~mL} \cdot \mathrm{min}^{-1}$, at $25{ }^{\circ} \mathrm{C}$ on an IC column, with UV detection $(\lambda=280 \mathrm{~nm})$. Retention times: 11.5 and $13.5 \mathrm{~min}$. 
${ }^{1} \mathrm{H}-\mathrm{NMR}$ of compound $3 \mathrm{~b}\left(400 \mathrm{MHz}, \mathrm{CDCl}_{3}\right)$

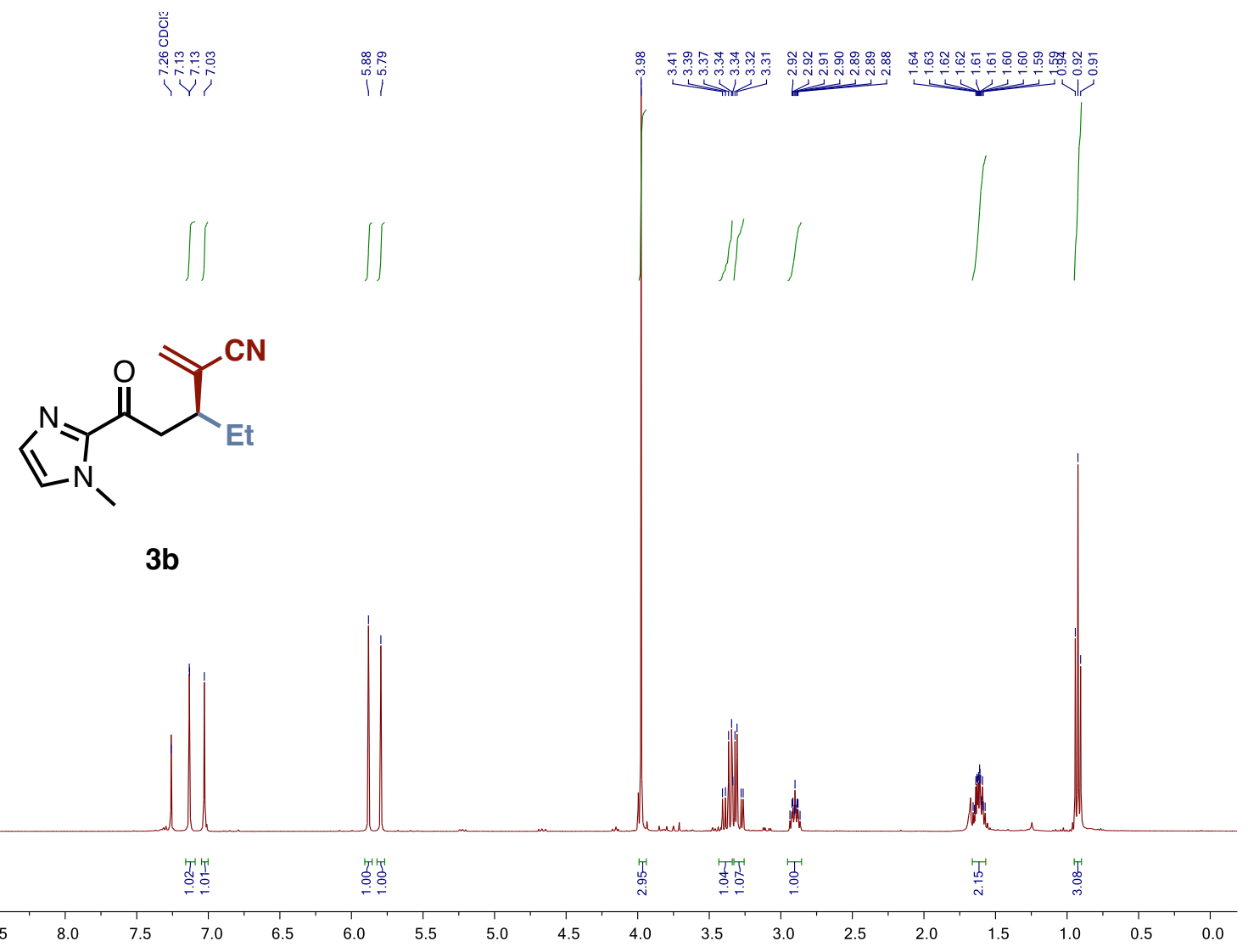

${ }^{13} \mathrm{C}-\mathrm{NMR}$ of compound $3 \mathrm{~b}\left(101 \mathrm{MHz}, \mathrm{CDCl}_{3}\right)$

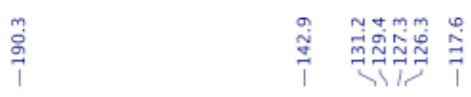

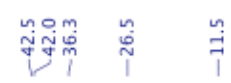



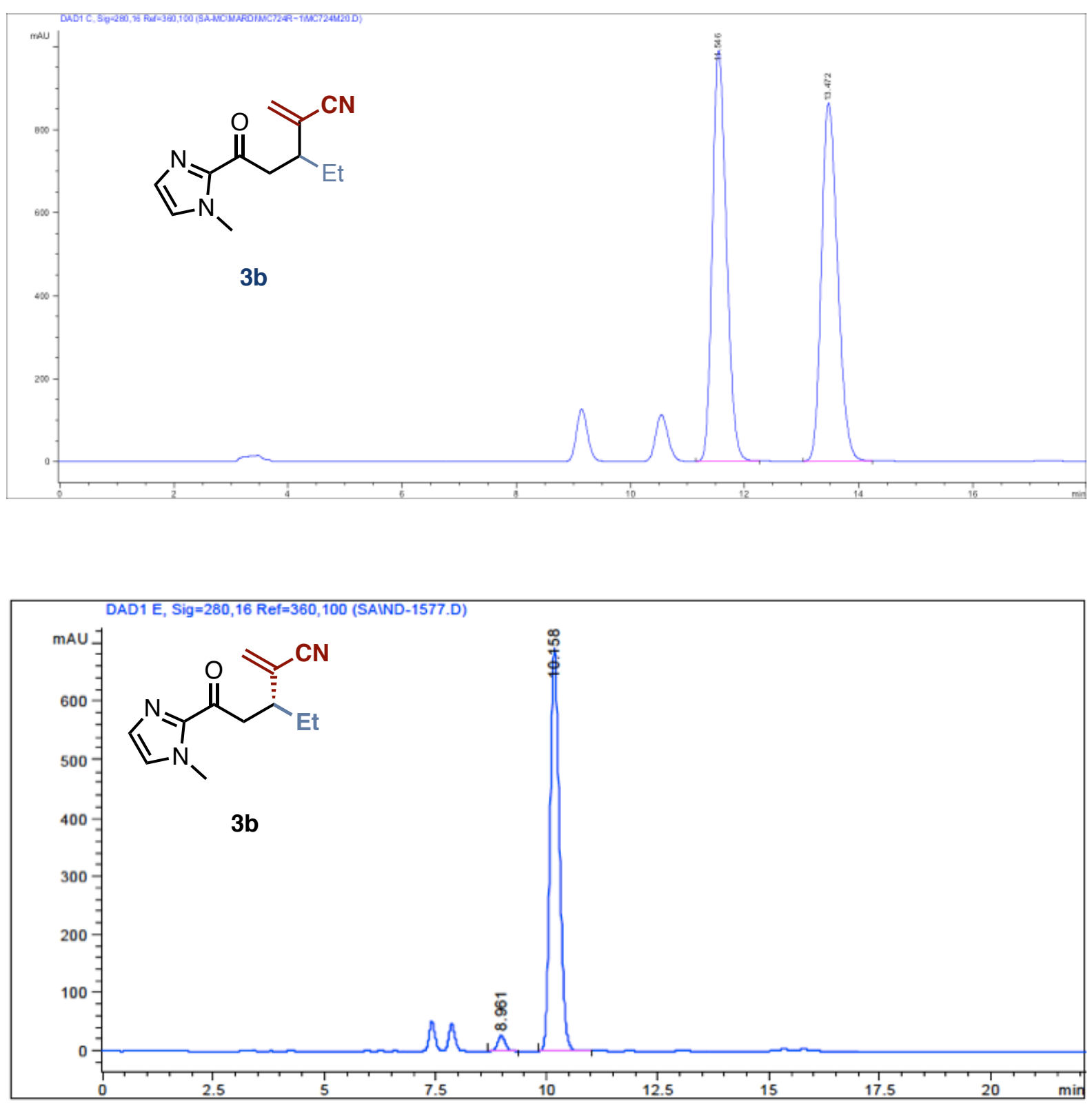

\begin{tabular}{|c|c|c|c|c|c|c|}
\hline $\begin{array}{c}\text { Peak } \\
\#\end{array}$ & $\begin{array}{c}\text { RetTime } \\
\text { [min] }\end{array}$ & Type & $\begin{array}{c}\text { Width } \\
\text { [min] }\end{array}$ & $\begin{array}{c}\text { Area } \\
{\left[\mathrm{mAU} \mathbf{U}^{*} \mathrm{~s}\right]}\end{array}$ & $\begin{array}{l}\text { Height } \\
\text { [mAU] }\end{array}$ & $\begin{array}{c}\text { Area } \\
\&\end{array}$ \\
\hline & & & & ---------- & ---------- & --------1 \\
\hline 1 & 8.961 & BB & 0.1791 & 306.07678 & 26.43696 & 2.9620 \\
\hline 2 & 10.158 & PB & 0.2250 & $1.00275 e 4$ & 692.16711 & 97.0380 \\
\hline
\end{tabular}




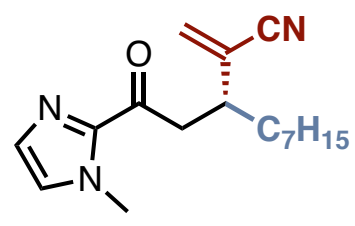

Molecular formula: $\mathrm{C}_{17} \mathrm{H}_{25} \mathrm{~N}_{3} \mathrm{O}$

$\mathbf{M W}=287.41 \mathrm{~g} \cdot \mathrm{mol}^{-1}$

The title compound was isolated as an amorphous solid (57 mg, $0.194 \mathrm{mmol}, 72 \%, 96 \%$ ee $)$ using general procedure F. Purified by flash column chromatography over silica gel $\left(\mathrm{Hex} / \mathrm{Et}_{2} \mathrm{O}=1: 1\right)$.

${ }^{1} \mathbf{H}$ NMR $\left(400 \mathrm{MHz}, \mathrm{CDCl}_{3}\right) \delta 7.13(\mathrm{~d}, J=0.9 \mathrm{~Hz}, 1 \mathrm{H}), 7.03(\mathrm{~d}, J=0.9 \mathrm{~Hz}, 1 \mathrm{H}), 5.86(\mathrm{~s}, 1 \mathrm{H})$, $5.79(\mathrm{~s}, 1 \mathrm{H}), 3.98(\mathrm{~s}, 3 \mathrm{H}), 3.37$ (dd, $J=17.1,8.4 \mathrm{~Hz}, 1 \mathrm{H}), 3.29$ (dd, $J=17.1,5.7 \mathrm{~Hz}, 1 \mathrm{H}), 2.98$ (tt, $J=8.4,5.7 \mathrm{~Hz}, 1 \mathrm{H}), 1.61-1.52(\mathrm{~m}, 2 \mathrm{H}), 1.33-1.21(\mathrm{~m}, 10 \mathrm{H}), 0.91-0.84(\mathrm{~m}, 3 \mathrm{H})$.

${ }^{13} \mathrm{C}$ NMR $\left(101 \mathrm{MHz} \mathrm{CDCl}_{3}\right) \delta 190.3,143.0,131.0,129.4,127.3,126.6,117.7,42.9,40.4,36.3$, $33.5,31.9,29.4,29.3,27.0,22.8,14.2$.

HRMS (ESI) m/z: [M+H] $]^{+}$calcd for $\mathrm{C}_{17} \mathrm{H}_{26} \mathrm{~N}_{3} \mathrm{O}$ 288.2076; found: 288.2083 .

IR $\left(\mathrm{cm}^{-1}\right): 2956,2929,2859,2228,1678,1467,1411,1292,1158$.

$[\boldsymbol{a}]_{D}^{20}=-19.6\left(c\right.$ 1.10, $\left.\mathrm{CH}_{2} \mathrm{Cl}_{2}\right)$

HPLC analysis: carried out using a mixture of hexane and isopropyl alcohol (85:15 isocratic), at $1 \mathrm{~mL} \cdot \mathrm{min}^{-1}$, at $25{ }^{\circ} \mathrm{C}$ on an IC column, with UV detection $(\lambda=280 \mathrm{~nm})$. Retention times: 10.5 and $13.8 \mathrm{~min}$. 
${ }^{1} \mathrm{H}-\mathrm{NMR}$ of compound $3 \mathrm{C}\left(400 \mathrm{MHz}, \mathrm{CDCl}_{3}\right)$

畒

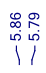

|

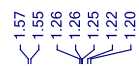

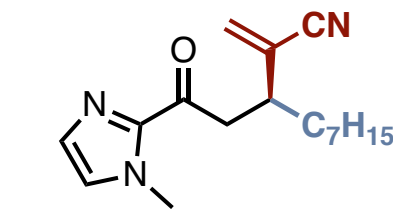

3c
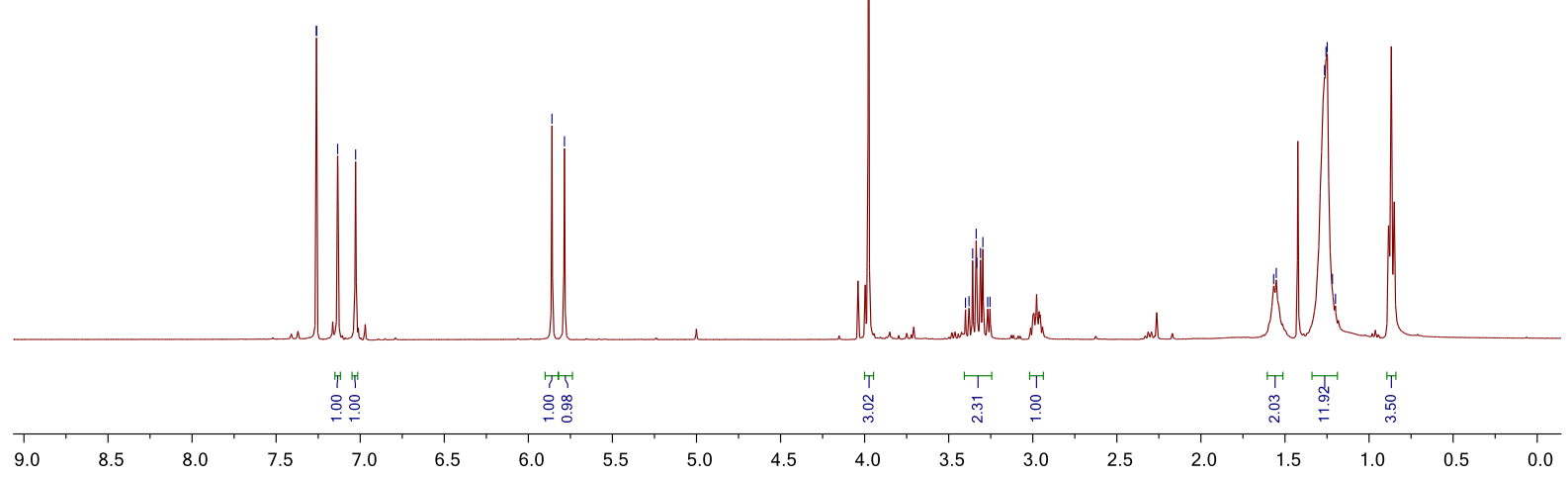

${ }^{13} \mathrm{C}-\mathrm{NMR}$ of compound $3 \mathrm{c}\left(101 \mathrm{MHz}, \mathrm{CDCl}_{3}\right)$

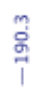

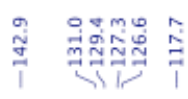

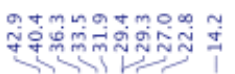
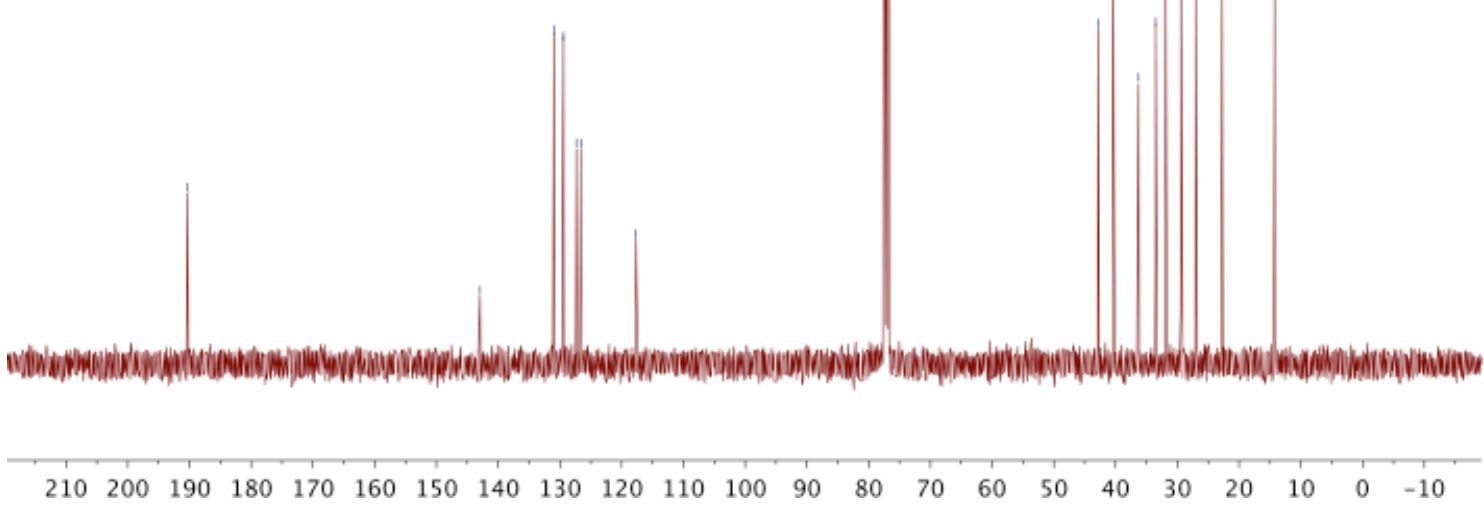

S56 

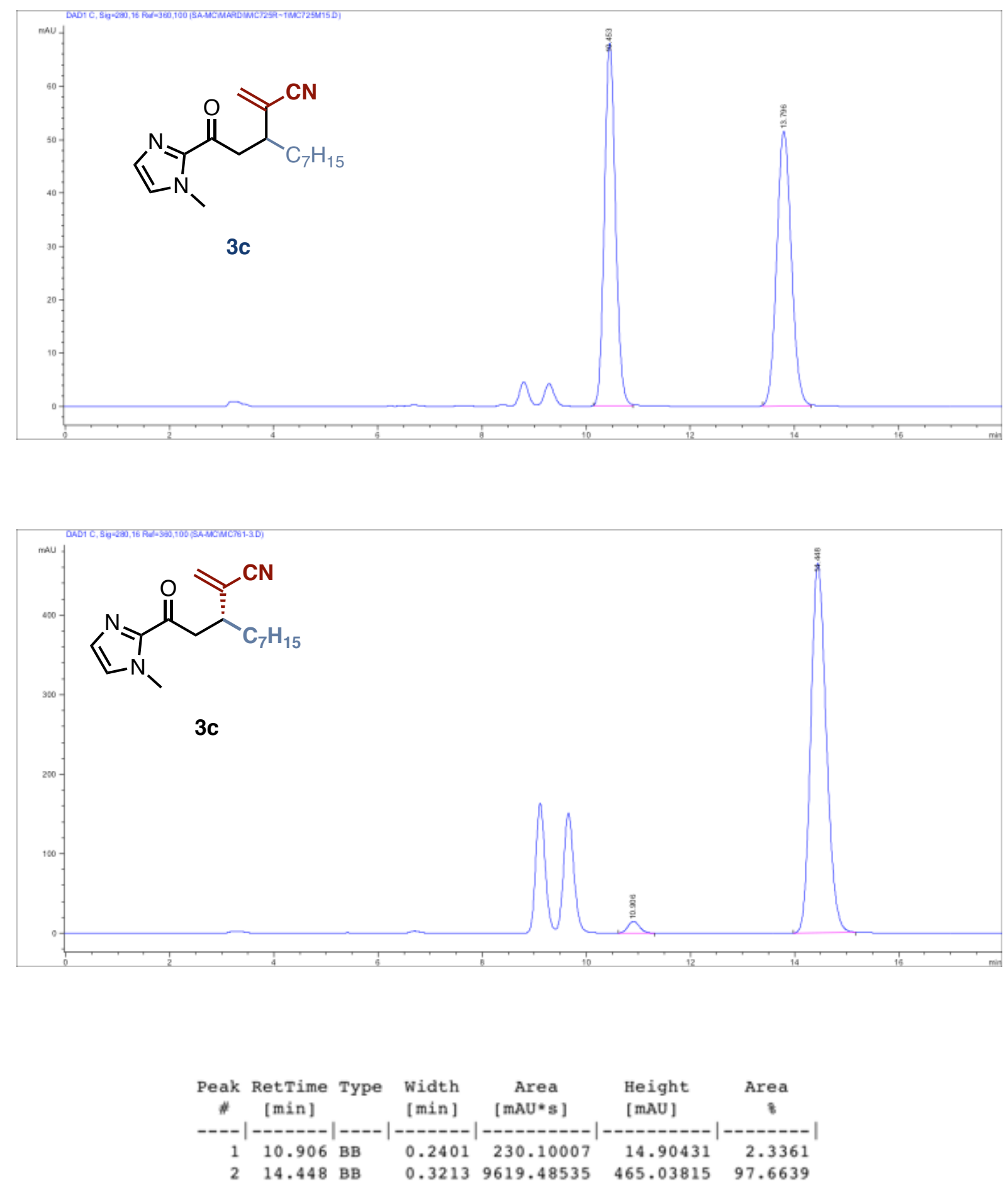


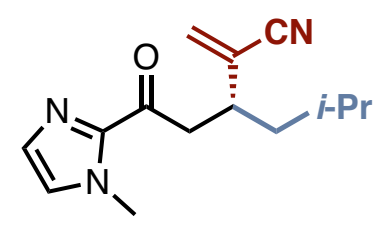

Molecular formula: $\mathrm{C}_{14} \mathrm{H}_{19} \mathrm{~N}_{3} \mathrm{O}$

$\mathrm{MW}=245.33 \mathrm{~g} \cdot \mathrm{mol}^{-1}$

The title compound was isolated as a clear oil $(28 \mathrm{mg}, 0.116 \mathrm{mmol}, 43 \%, 94 \%$ ee $)$ using general procedure F. Purified by flash column chromatography over silica gel $(\mathrm{Hex} /$ EtOAc $=6: 4)$.

${ }^{1}$ H NMR $\left(400 \mathrm{MHz}, \mathrm{CDCl}_{3}\right) \delta 7.13(\mathrm{~d}, J=0.9 \mathrm{~Hz}, 1 \mathrm{H}), 7.03(\mathrm{~d}, J=0.9 \mathrm{~Hz}, 1 \mathrm{H}), 5.86(\mathrm{~s}, 1 \mathrm{H}), 5.81$ (s, 1H), $3.98(\mathrm{~s}, 3 \mathrm{H}), 3.37$ (dd, $J=17.2,8.6 \mathrm{~Hz}, 1 \mathrm{H}), 3.26(\mathrm{dd}, J=17.2,5.4 \mathrm{~Hz}, 1 \mathrm{H}), 3.15(\mathrm{~m}$, 1H), $1.65-1.54(m, 2 H), 1.34(m, 1 H), 0.93(d, J=6.4 \mathrm{~Hz}, 3 \mathrm{H}), 0.91(\mathrm{~d}, J=6.4 \mathrm{~Hz}, 3 \mathrm{H})$.

${ }^{13} \mathrm{C}$ NMR $\left(101 \mathrm{MHz}, \mathrm{CDCl}_{3}\right) \delta 190.3,142.9,131.0,129.4,127.3,126.7,117.6,43.2,42.6,38.3$, $36.3,25.4,23.3,21.7$.

HRMS (ESI) m/z: [M+H] $]^{+}$calcd for $\mathrm{C}_{14} \mathrm{H}_{20} \mathrm{~N}_{3} \mathrm{O}$ 246.1606; found: 246.1502 .

IR $\left(\mathrm{cm}^{-1}\right):$ 2959, 2929, 2872, 2224, 1678, 1471, 1413, 1292, 1158.

$[\boldsymbol{a}]_{\boldsymbol{D}}^{\mathbf{2 0}}=-80.6\left(c 0.23, \mathrm{CH}_{2} \mathrm{Cl}_{2}\right)$

HPLC analysis: carried out using a mixture of hexane and isopropyl alcohol (80:20 isocratic), at $1 \mathrm{~mL} \cdot \mathrm{min}^{-1}$, at $25{ }^{\circ} \mathrm{C}$ on an IC column, with UV detection $(\lambda=280 \mathrm{~nm})$. Retention times: 7.0 and $8.7 \mathrm{~min}$. 


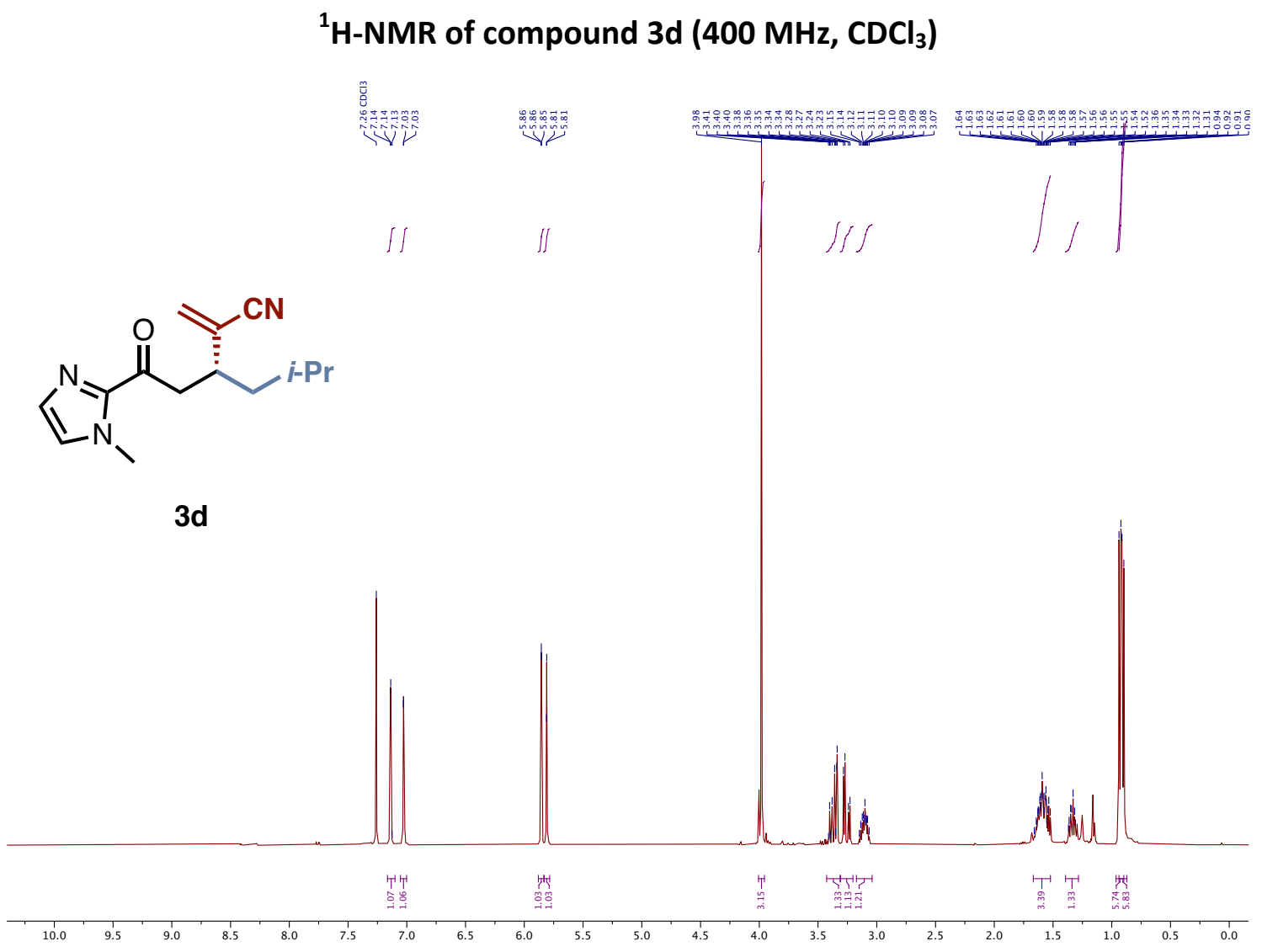

${ }^{13} \mathrm{C}-\mathrm{NMR}$ of compound 3d (101 $\left.\mathrm{MHz}, \mathrm{CDCl}_{3}\right)$

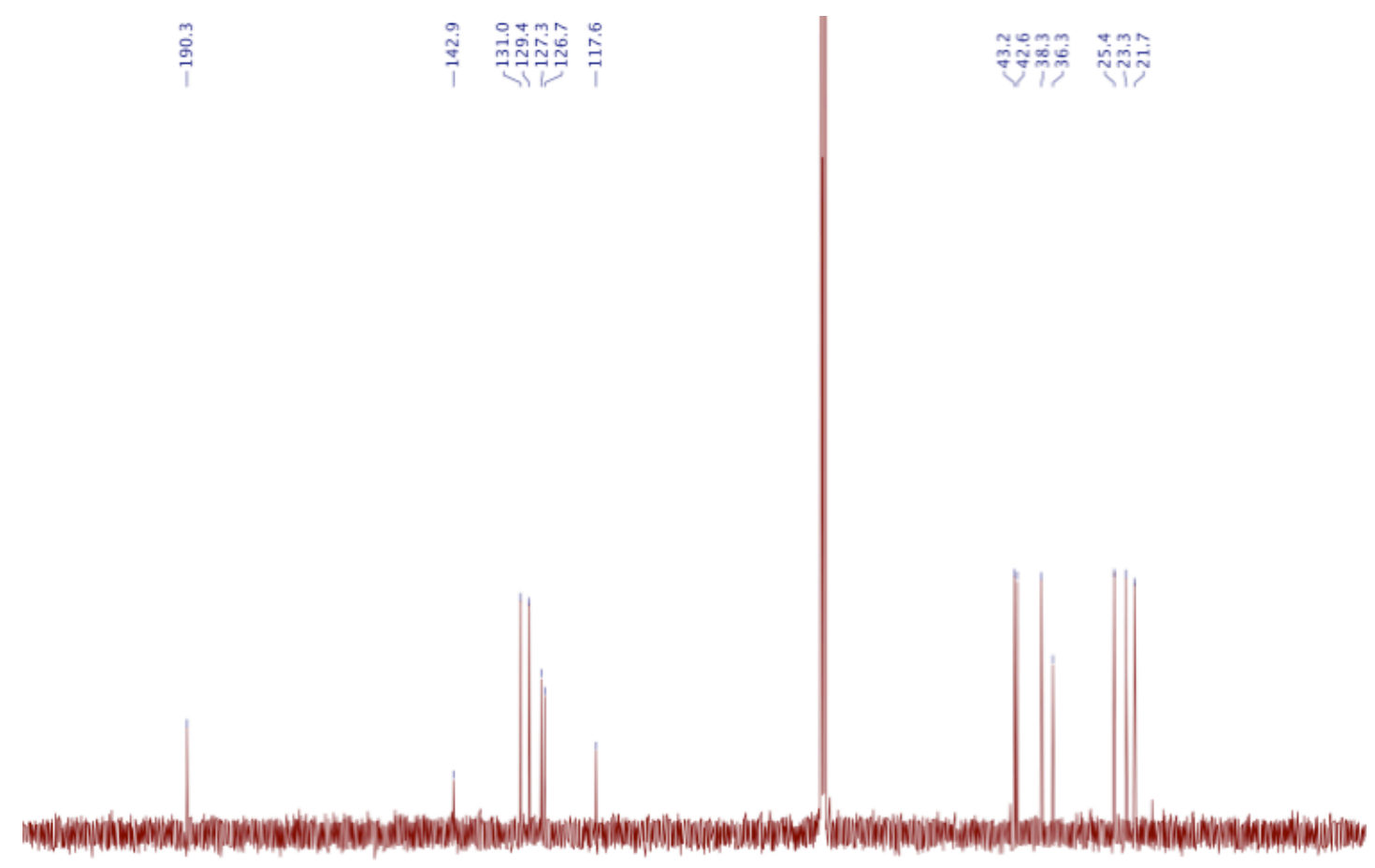

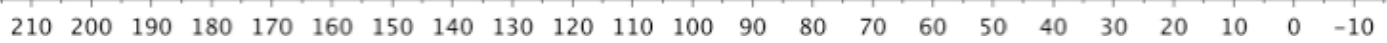



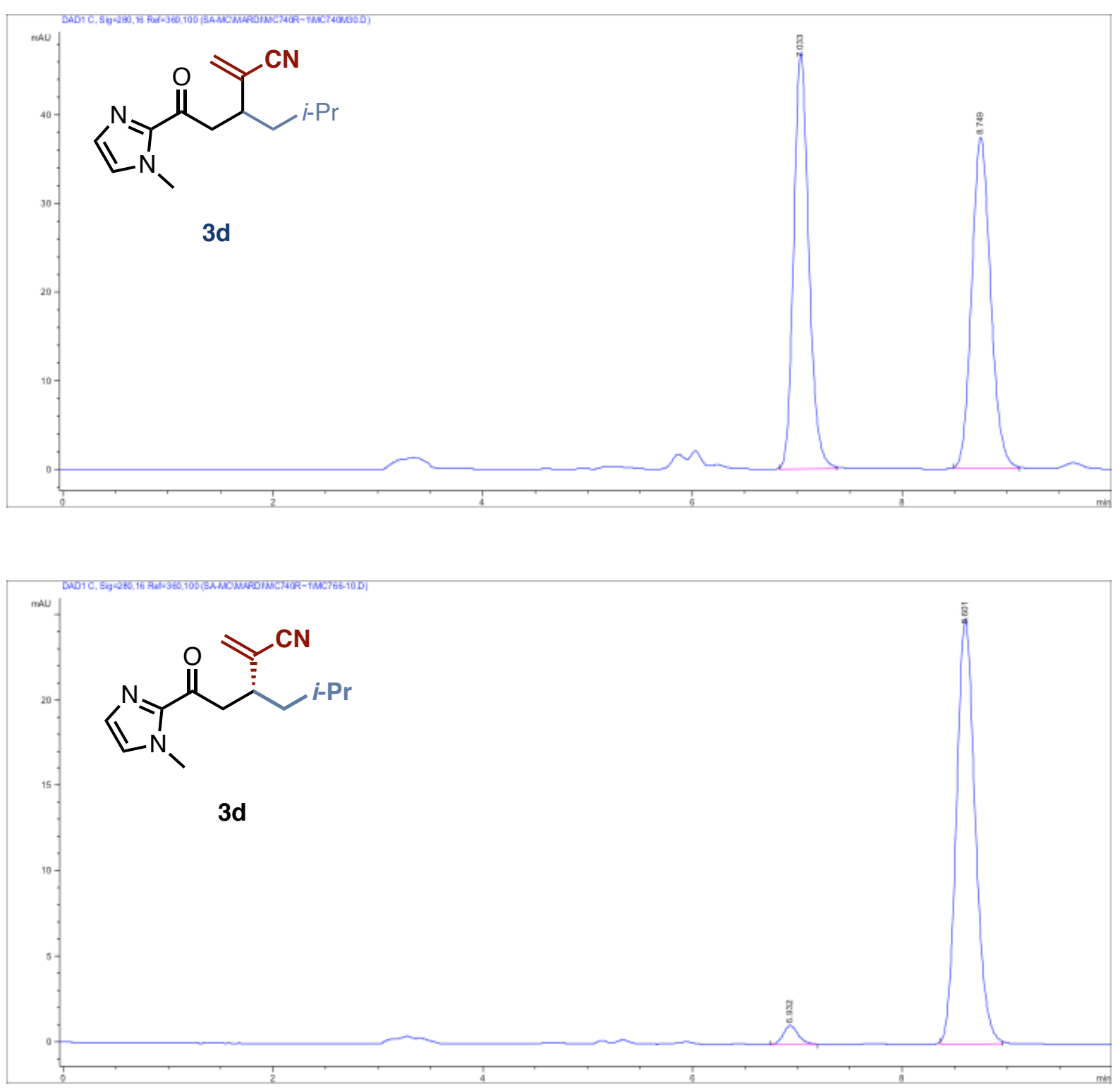

\begin{tabular}{|c|c|c|c|c|c|c|}
\hline $\begin{array}{c}\text { Peak } \\
\#\end{array}$ & $\begin{array}{c}\text { RetTime } \\
\text { [min] }\end{array}$ & Type & $\begin{array}{l}\text { Width } \\
\text { [min] }\end{array}$ & $\begin{array}{c}\text { Area } \\
{\left[\mathrm{mAU}{ }^{*} \mathrm{~s}\right]}\end{array}$ & $\begin{array}{l}\text { Height } \\
\text { [mAU] }\end{array}$ & $\begin{array}{c}\text { Area } \\
\frac{8}{8}\end{array}$ \\
\hline-- & & & & 年- & & \\
\hline 1 & 6.932 & PB & 0.1540 & 10.88285 & 1.09438 & 3.3916 \\
\hline 2 & 8.601 & BB & 0.1934 & 309.99725 & 24.87632 & 96.6084 \\
\hline
\end{tabular}


3-(Cyclohexylmethyl)-5-(1-methyl-1H-imidazol-2-yl)-2-methylene-5-oxopentanenitrile (3e)

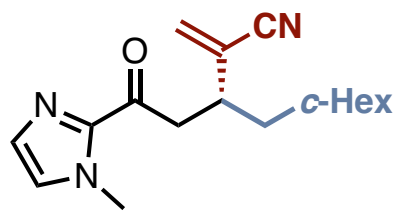

Molecular formula: $\mathrm{C}_{17} \mathrm{H}_{23} \mathrm{~N}_{3} \mathrm{O}$

$\mathbf{M W}=285.39 \mathrm{~g} \cdot \mathrm{mol}^{-1}$

The title compound was isolated as an amorphous solid (41 mg, $0.143 \mathrm{mmol}, 53 \%, 95 \%$ ee $)$ using general procedure F. Purified by flash column chromatography over silica gel $($ Hex/EtOAc/Acetone $=7: 2: 1)$.

${ }^{1} \mathbf{H}$ NMR $\left(400 \mathrm{MHz}, \mathrm{CDCl}_{3}\right) \delta 7.14(\mathrm{~d}, J=1.0 \mathrm{~Hz}, 1 \mathrm{H}), 7.03(\mathrm{~d}, 1.0 \mathrm{~Hz}, 1 \mathrm{H}), 5.86(\mathrm{~d}, J=0.6 \mathrm{~Hz}$, $1 \mathrm{H}), 5.80(\mathrm{~d}, J=0.7 \mathrm{~Hz}, 1 \mathrm{H}), 3.98(\mathrm{~s}, 3 \mathrm{H}), 3.37(\mathrm{dd}, J=17.2,8.6 \mathrm{~Hz}, 1 \mathrm{H}), 3.25(\mathrm{dd}, J=17.2$, $5.3 \mathrm{~Hz}, 1 \mathrm{H}), 1.77-1.60(\mathrm{~m}, 6 \mathrm{H}), 1.30-1.11(\mathrm{~m}, 6 \mathrm{H}), 0.98-0.83(\mathrm{~m}, 3 \mathrm{H})$.

${ }^{13} \mathrm{C}$ NMR $\left(101 \mathrm{MHz}, \mathrm{CDCl}_{3}\right) \delta 190.2,130.8,129.3,127.2,126.7,117.5,43.0,41.0,37.3,34.7$, $33.7,32.5,30.3,26.5,26.2,26.1$.

HRMS (ESI) m/z: [M+H] $]^{+}$calcd for $\mathrm{C}_{17} \mathrm{H}_{24} \mathrm{~N}_{3} \mathrm{O}$ 286.1919; found: 286.1925 .

IR $\left(\mathrm{cm}^{-1}\right): 2924,2853,2224,1743,1678,1451,1411,1292,1158$.

$[\boldsymbol{a}]_{\boldsymbol{D}}^{\mathbf{2 0}}=-39.2\left(c 0.50, \mathrm{CH}_{2} \mathrm{Cl}_{2}\right)$

HPLC analysis: carried out using a mixture of hexane and isopropyl alcohol (80:20 isocratic), at $1 \mathrm{~mL} \cdot \mathrm{min}^{-1}$, at $30{ }^{\circ} \mathrm{C}$ on an IC column, with UV detection $(\lambda=280 \mathrm{~nm})$. Retention times: 7.69 and $10.29 \mathrm{~min}$. 
${ }^{1} \mathrm{H}-\mathrm{NMR}$ of compound $3 \mathrm{e}\left(400 \mathrm{MHz}, \mathrm{CDCl}_{3}\right)$

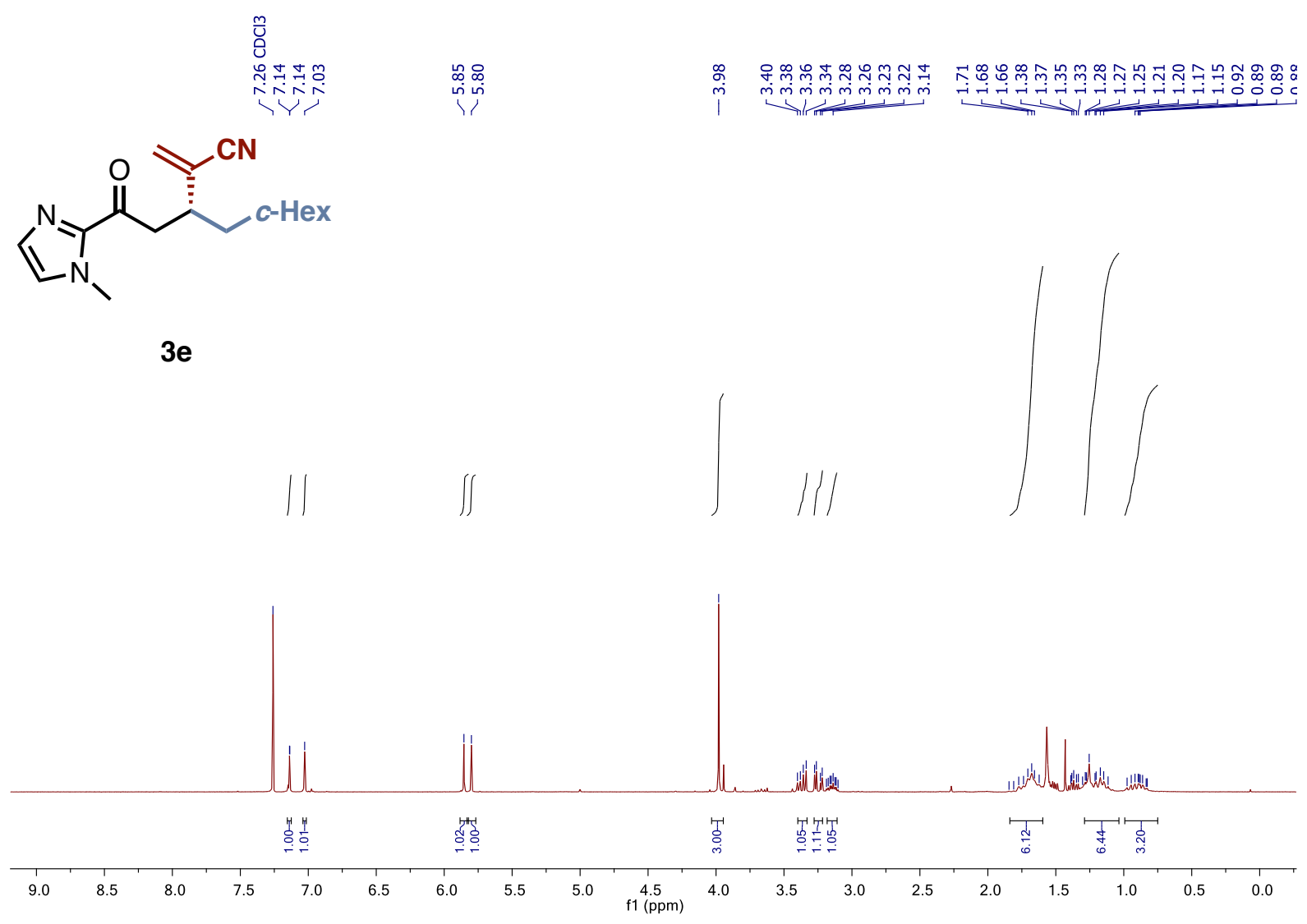

${ }^{13} \mathrm{C}-\mathrm{NMR}$ of compound $3 \mathrm{e}\left(101 \mathrm{MHz}, \mathrm{CDCl}_{3}\right)$
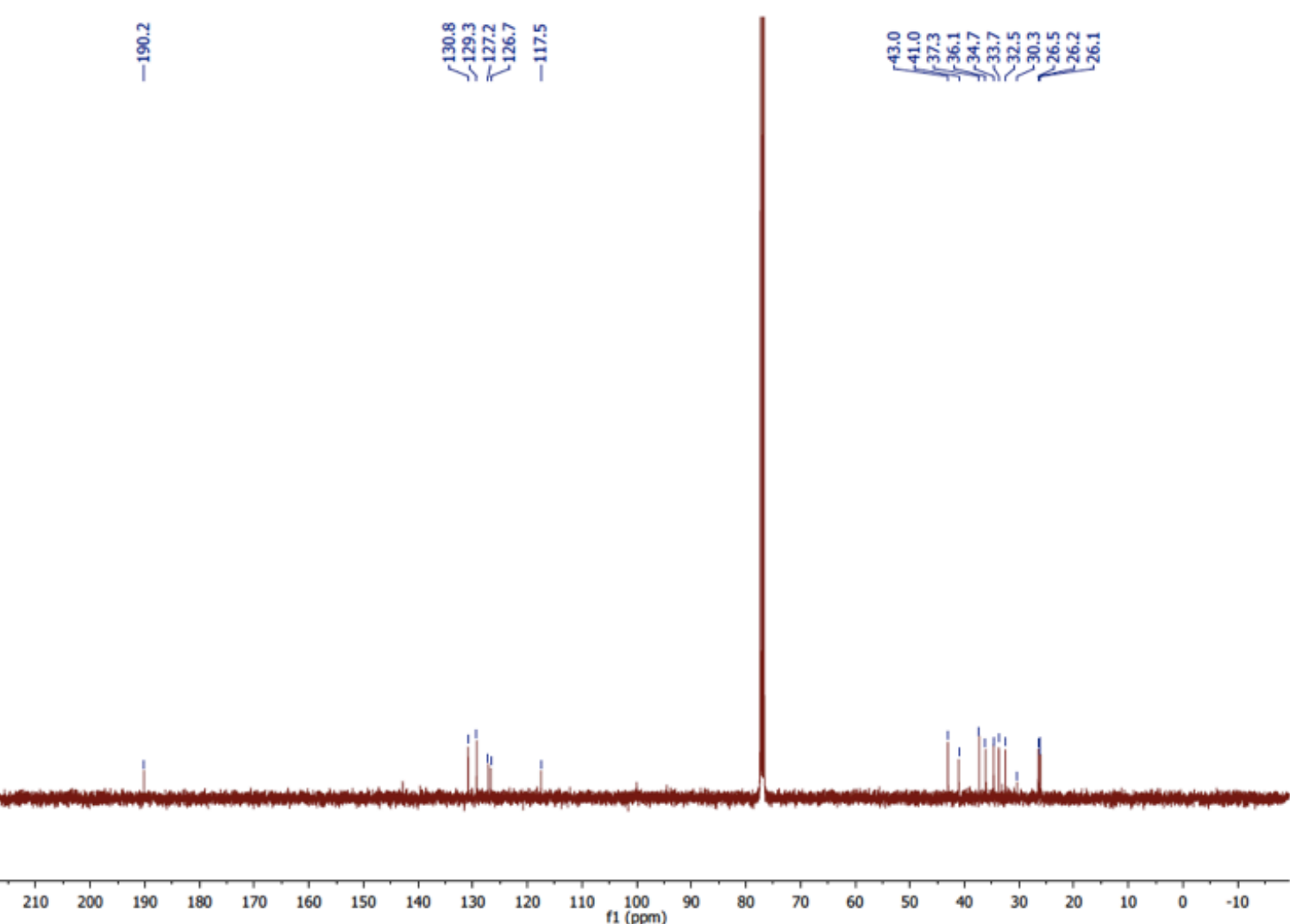

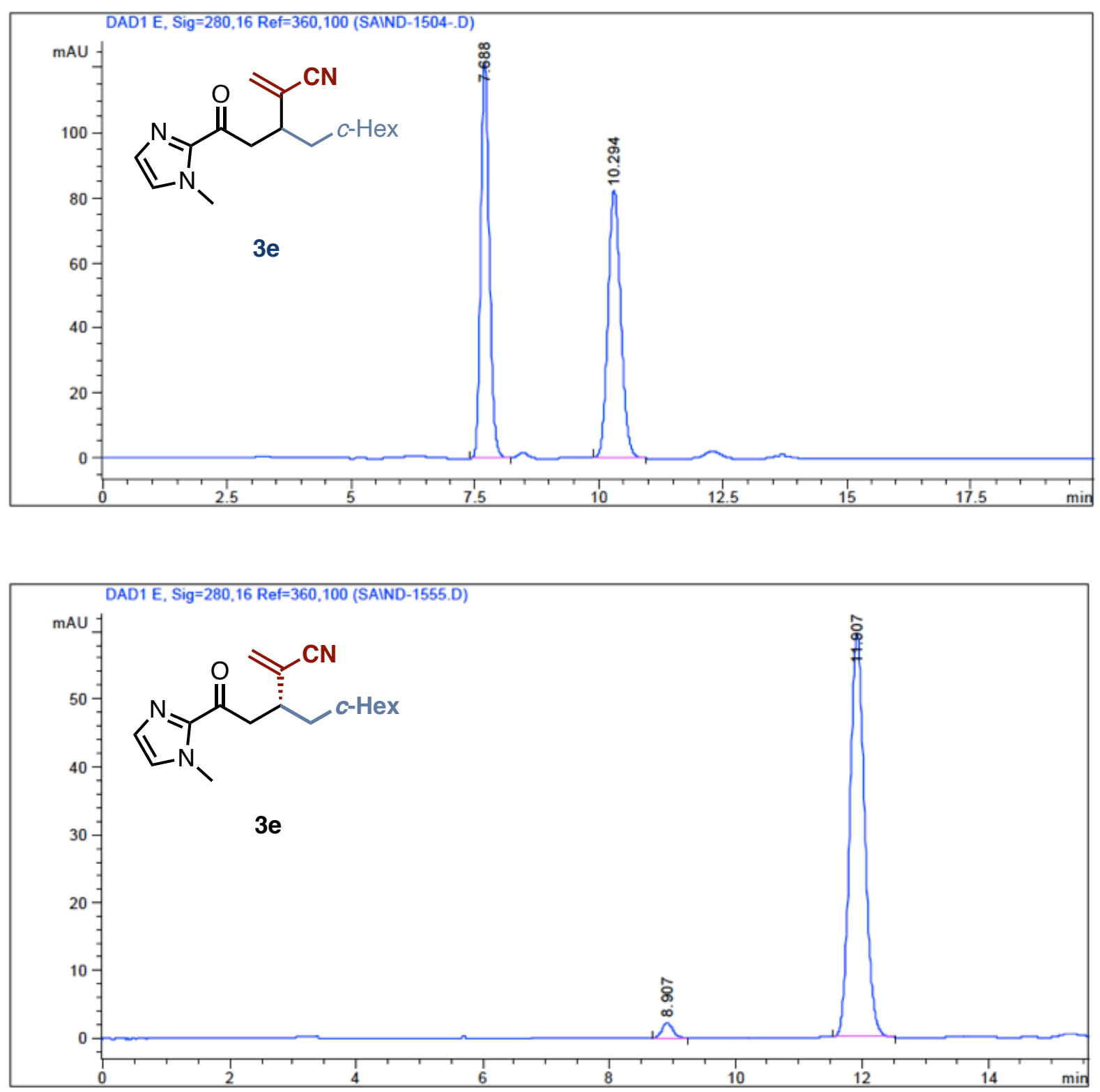

\begin{tabular}{|c|c|c|c|c|c|c|}
\hline $\begin{array}{c}\text { Peak } \\
\#\end{array}$ & $\begin{array}{c}\text { RetTime } \\
\text { [min] }\end{array}$ & Type & $\begin{array}{c}\text { width } \\
\text { [min] }\end{array}$ & $\begin{array}{c}\text { Area } \\
{\left[\mathrm{mAU}^{*} \mathrm{~S}\right]}\end{array}$ & $\begin{array}{l}\text { Height } \\
\text { [mAU] }\end{array}$ & $\begin{array}{c}\text { Area } \\
\frac{\%}{6}\end{array}$ \\
\hline-- & - - - - - & & ------ & --------- & $---n--n--$ & $-----n--$ \\
\hline 1 & 8.907 & $\mathrm{BB}$ & 0.1839 & 27.05397 & 2.25646 & 2.7180 \\
\hline 2 & 11.907 & BB & 0.2519 & 968.29352 & 59.49014 & 97.2820 \\
\hline
\end{tabular}




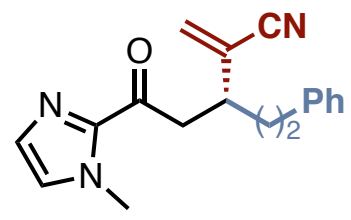

Molecular formula: $\mathrm{C}_{18} \mathrm{H}_{19} \mathrm{~N}_{3} \mathrm{O}$

$\mathbf{M W}=293.37 \mathrm{~g} \cdot \mathrm{mol}^{-1}$

The title compound was isolated as a clear oil (50 mg, $0.170 \mathrm{mmol}, 63 \%, 93 \%$ ee $)$ using general procedure F. Purified by flash column chromatography over silica gel $\left(\mathrm{Hex} / \mathrm{Et}_{2} \mathrm{O}=3: 7\right)$.

${ }^{1} \mathbf{H}$ NMR $\left(400 \mathrm{MHz}, \mathrm{CDCl}_{3}\right) \delta 7.30-7.25(\mathrm{~m}, 2 \mathrm{H}), 7.18(\mathrm{td}, J=6.6,1.5 \mathrm{~Hz}, 2 \mathrm{H}), 7.14(\mathrm{~d}$, $J=1.0 \mathrm{~Hz}, 1 \mathrm{H}), 7.03(\mathrm{~d}, 1.0 \mathrm{~Hz}, 1 \mathrm{H}), 5.93(\mathrm{~d}, J=0.6 \mathrm{~Hz}, 1 \mathrm{H}), 5.82(\mathrm{~s}, 1 \mathrm{H}), 3.97(\mathrm{~s}, 3 \mathrm{H}), 3.43$ (dd, $J=17.1,8.4 \mathrm{~Hz}, 1 \mathrm{H}), 3.33(\mathrm{dd}, J=17.1,5.6 \mathrm{~Hz}, 1 \mathrm{H}), 3.04(\mathrm{tt}, J=7.6,6.0 \mathrm{~Hz}, 1 \mathrm{H}), 2.70(\mathrm{~m}$, $1 \mathrm{H}), 2.58(\mathrm{dd}, J=13.8,8.2 \mathrm{~Hz}, 1 \mathrm{H}), 1.95-1.89(\mathrm{~m}, 2 \mathrm{H})$.

${ }^{13} \mathrm{C}$ NMR $\left(101 \mathrm{MHz}, \mathrm{CDCl}_{3}\right) \delta 189.8,142.7,141.0,131.5,129.3,128.5,128.4,126.1,117.4$, $42.6,39.9,36.1,34.9,33.1,30.3$.

HRMS (ESI) m/z: [M+Na] $]^{+}$calcd for $\mathrm{C}_{18} \mathrm{H}_{19} \mathrm{~N}_{3} \mathrm{ONa} 316.1426$; found: 316.1429.

IR $\left(\mathrm{cm}^{-1}\right): 2928,2861,2224,1739,1676,1456,1410,1292,1158$.

$[a]_{D}^{20}=-44.1\left(c 1.23, \mathrm{CH}_{2} \mathrm{Cl}_{2}\right)$

HPLC analysis: carried out using a mixture of hexane and isopropyl alcohol (80:20 isocratic), at $1 \mathrm{~mL} \cdot \mathrm{min}^{-1}$, at $30{ }^{\circ} \mathrm{C}$ on an IC column, with UV detection $(\lambda=280 \mathrm{~nm})$. Retention times: 11.24 and $13.52 \mathrm{~min}$. 
${ }^{1} \mathrm{H}-\mathrm{NMR}$ of compound $3 \mathrm{f}\left(400 \mathrm{MHz}, \mathrm{CDCl}_{3}\right)$

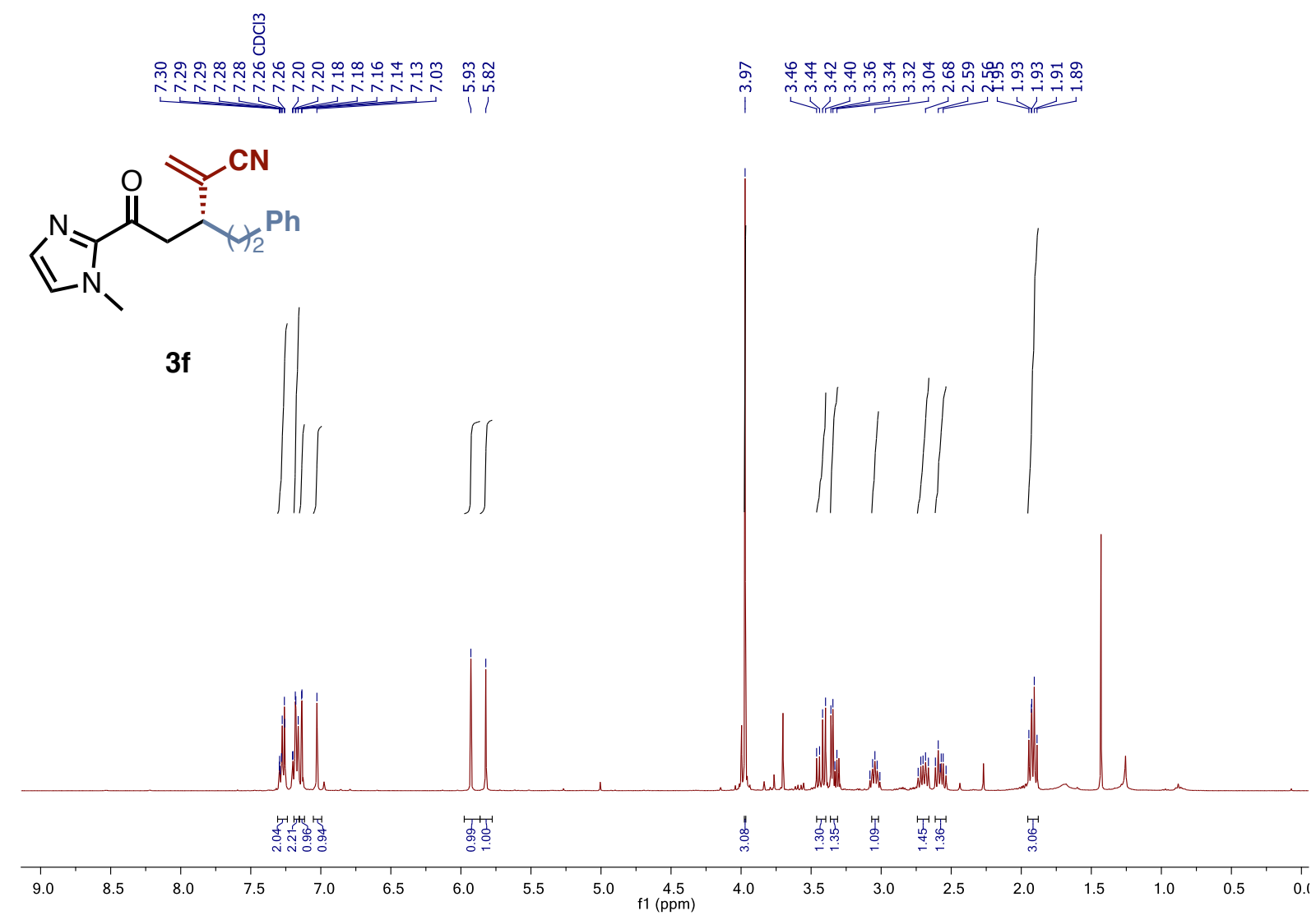

${ }^{13} \mathrm{C}-\mathrm{NMR}$ of compound $3 \mathrm{f}\left(101 \mathrm{MHz}, \mathrm{CDCl}_{3}\right)$

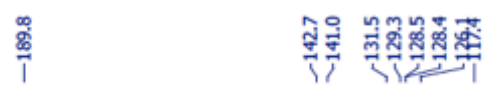

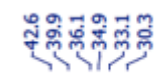

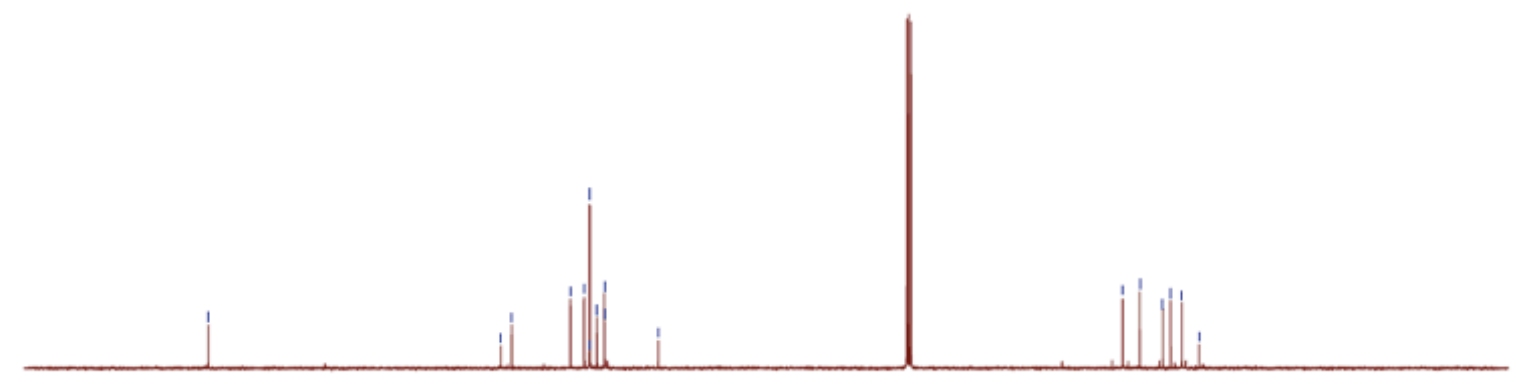

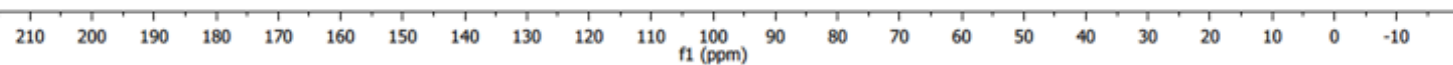



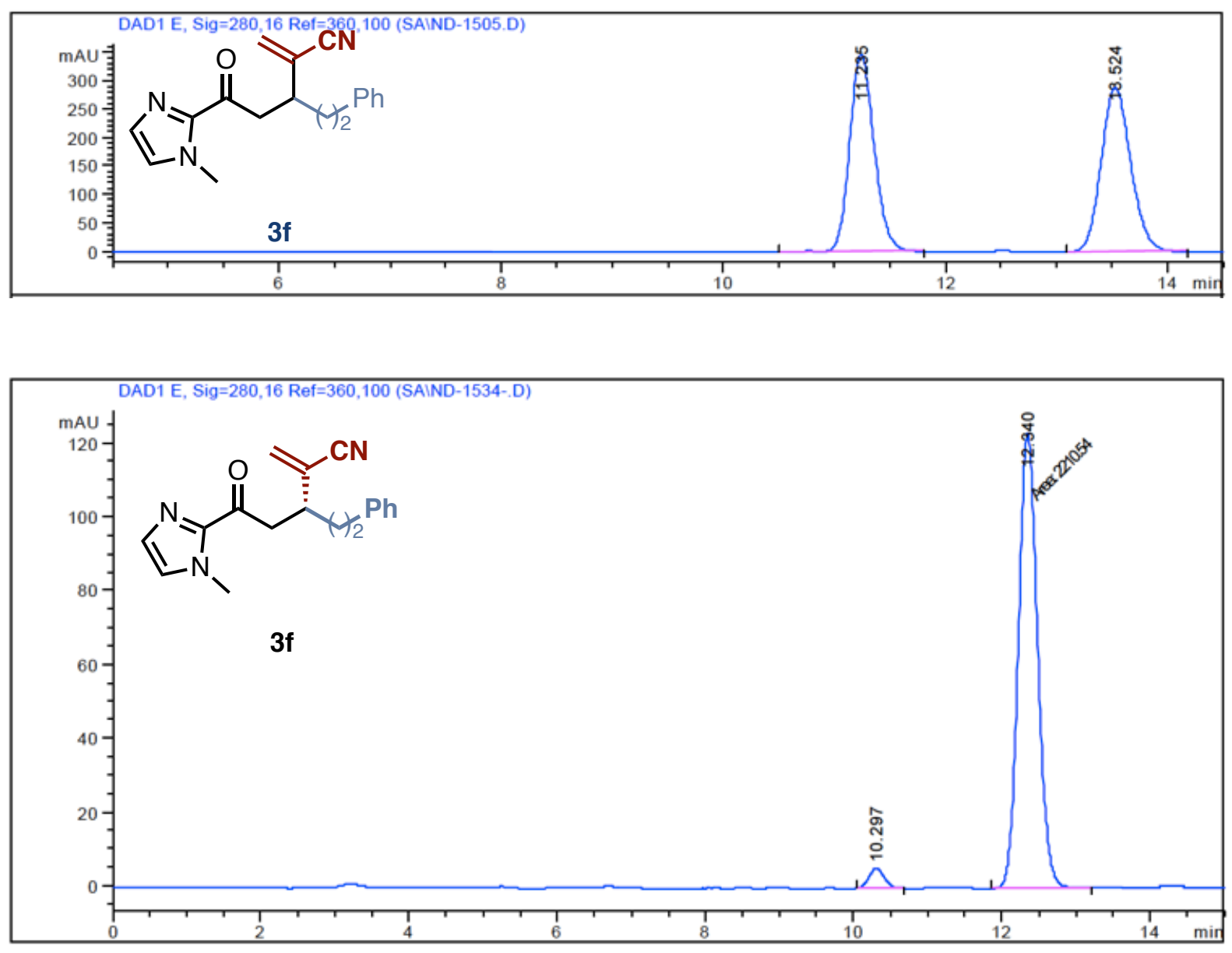

\begin{tabular}{|c|c|c|c|c|c|c|}
\hline $\begin{array}{c}\text { Peak } \\
\#\end{array}$ & $\begin{array}{c}\text { RetTime } \\
\text { [min] }\end{array}$ & Type & $\begin{array}{l}\text { Width } \\
\text { [min] }\end{array}$ & $\begin{array}{c}\text { Area } \\
{\left[\mathrm{mAU}^{\star} \mathrm{s}\right]}\end{array}$ & $\begin{array}{l}\text { Height } \\
\text { [mAU] }\end{array}$ & $\begin{array}{c}\text { Area } \\
\text { \& }\end{array}$ \\
\hline$x^{2}$ & & & 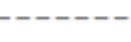 & ---------- & 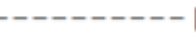 & 1 \\
\hline 1 & 10.297 & BB & 0.2248 & 78.43433 & 5.42037 & 3.4266 \\
\hline 2 & 12.340 & MM & 0.2992 & 2210.53784 & 123.12493 & 96.5734 \\
\hline
\end{tabular}




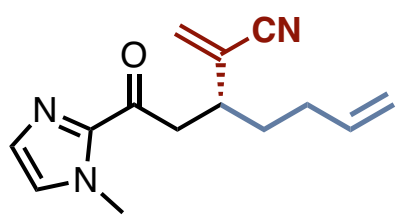

Molecular formula: $\mathrm{C}_{14} \mathrm{H}_{17} \mathrm{~N}_{3} \mathrm{O}$

$\mathbf{M W}=243.31 \mathrm{~g} \cdot \mathrm{mol}^{-1}$

The title compound was isolated as yellow oil (38 mg, $0.159 \mathrm{mmol}, 59 \%, 95 \%$ ee $)$ using general procedure F. Purified by flash column chromatography over silica gel $(\mathrm{Hex} / \mathrm{EtOAc}=6: 4)$.

${ }^{1}$ H NMR $\left(400 \mathrm{MHz}^{\mathrm{C}} \mathrm{CDCl}_{3}\right) \delta 7.14(\mathrm{~d}, J=0.9 \mathrm{~Hz}, 1 \mathrm{H}), 7.03(\mathrm{~d}, J=0.9 \mathrm{~Hz}, 1 \mathrm{H}), 5.89$ (d, $J=0.6 \mathrm{~Hz}, 1 \mathrm{H}), 5.82-5.72(\mathrm{~m}, 2 \mathrm{H}), 5.05-4.98(\mathrm{~m}, 2 \mathrm{H}), 3.98(\mathrm{~s}, 3 \mathrm{H}), 3.40(\mathrm{dd}, J=17.2,8.4 \mathrm{~Hz}$, $1 \mathrm{H}), 3.30(\mathrm{dd}, J=17.2,5.7 \mathrm{~Hz}, 1 \mathrm{H}), 3.03(\mathrm{tt}, J=8.4,5.7 \mathrm{~Hz}, 1 \mathrm{H}), 2.13(\mathrm{~m}, 1 \mathrm{H}), 2.04(\mathrm{~m}, 1 \mathrm{H})$, $1.71-1.65(\mathrm{~m}, 2 \mathrm{H})$.

${ }^{13} \mathrm{C}$ NMR $\left(101 \mathrm{MHz}, \mathrm{CDCl}_{3}\right) \delta 189.9,137.2,131.3,129.3,127.2,126.0,117.4,117.4,115.6$, $42.6,39.6,36.1,32.3,30.9$.

HRMS (ESI) m/z: [M+H] calcd for $\mathrm{C}_{14} \mathrm{H}_{18} \mathrm{~N}_{3} \mathrm{O}$ 244.1450; found: 244.1427 .

IR $\left(\mathrm{cm}^{-1}\right): 3295,2931,2857,2222,1678,1411,1262$

$[a]_{D}^{20}=-22.8\left(c\right.$ 1.22, $\left.\mathrm{CH}_{2} \mathrm{Cl}_{2}\right)$

HPLC analysis: carried out using a mixture of hexane and isopropyl alcohol (85:15 isocratic) at $1 \mathrm{~mL} \cdot \mathrm{min}^{-1}$, at $30{ }^{\circ} \mathrm{C}$ on an IC column, with UV detection $(\lambda=280 \mathrm{~nm})$. Retention times: 8.41 and $9.79 \mathrm{~min}$. 
${ }^{1} \mathrm{H}-\mathrm{NMR}$ of compound $3 \mathrm{~g}\left(400 \mathrm{MHz}, \mathrm{CDCl}_{3}\right)$

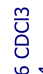

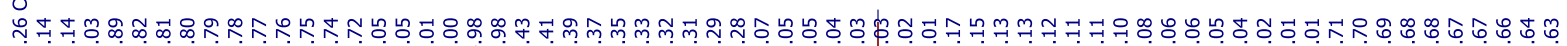

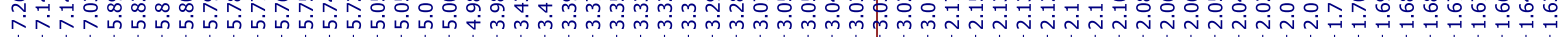<smiles>C=CCC[C@H](CC(=O)c1nccn1C)C(=C)C#N</smiles>

$3 \mathbf{g}$

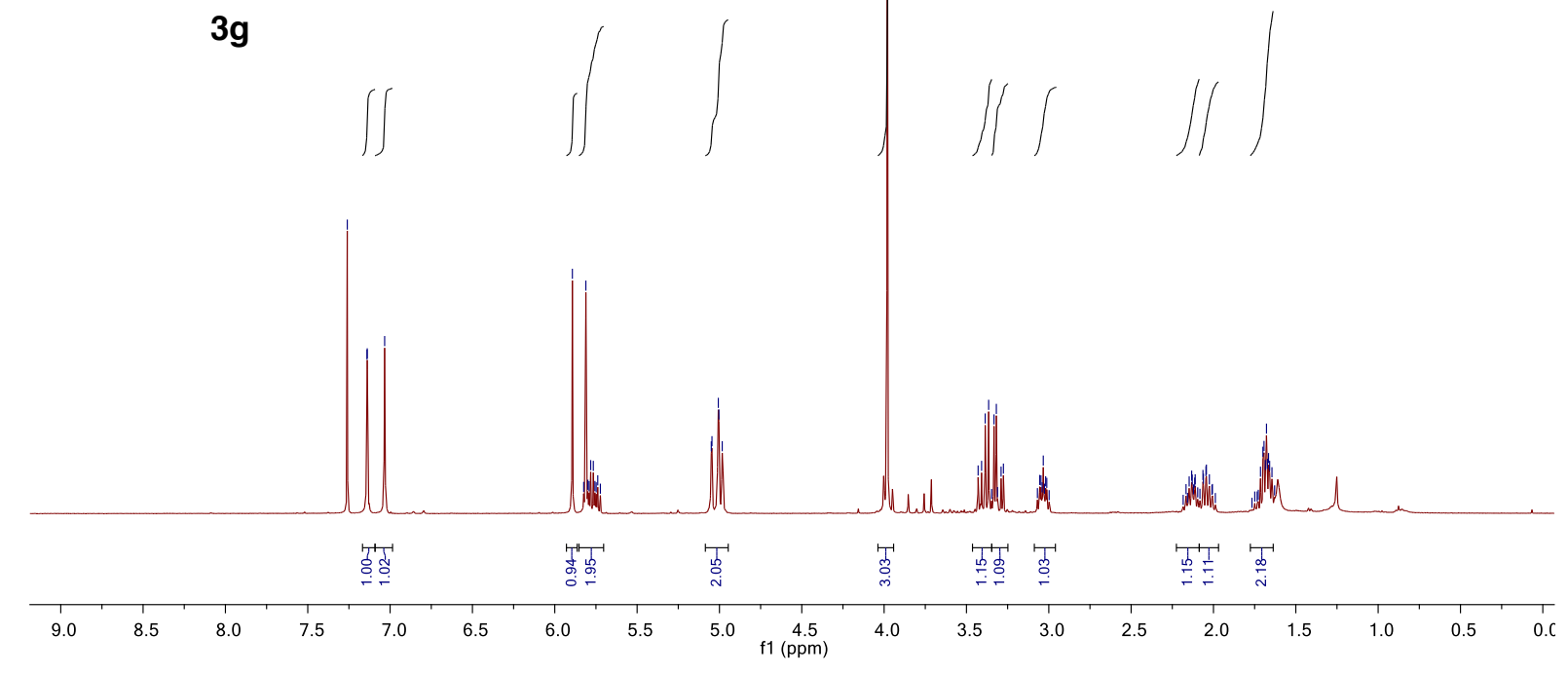

${ }^{13} \mathrm{C}-\mathrm{NMR}$ of compound $3 \mathrm{~g}\left(101 \mathrm{MHz}, \mathrm{CDCl}_{3}\right)$

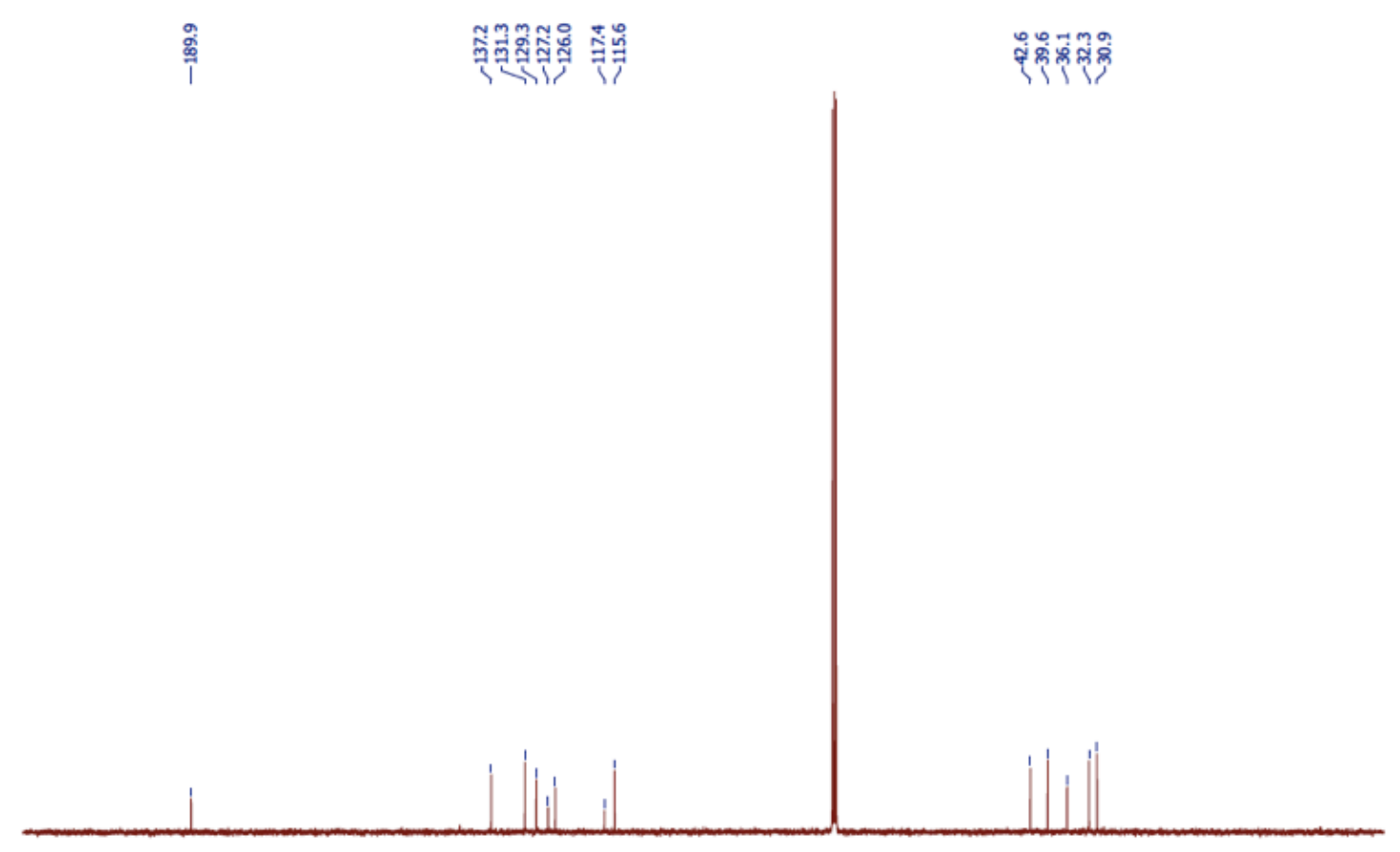

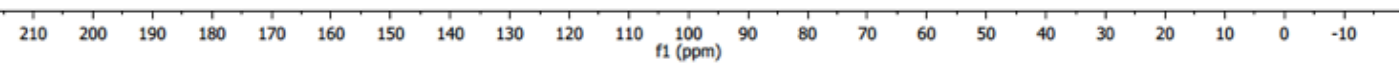



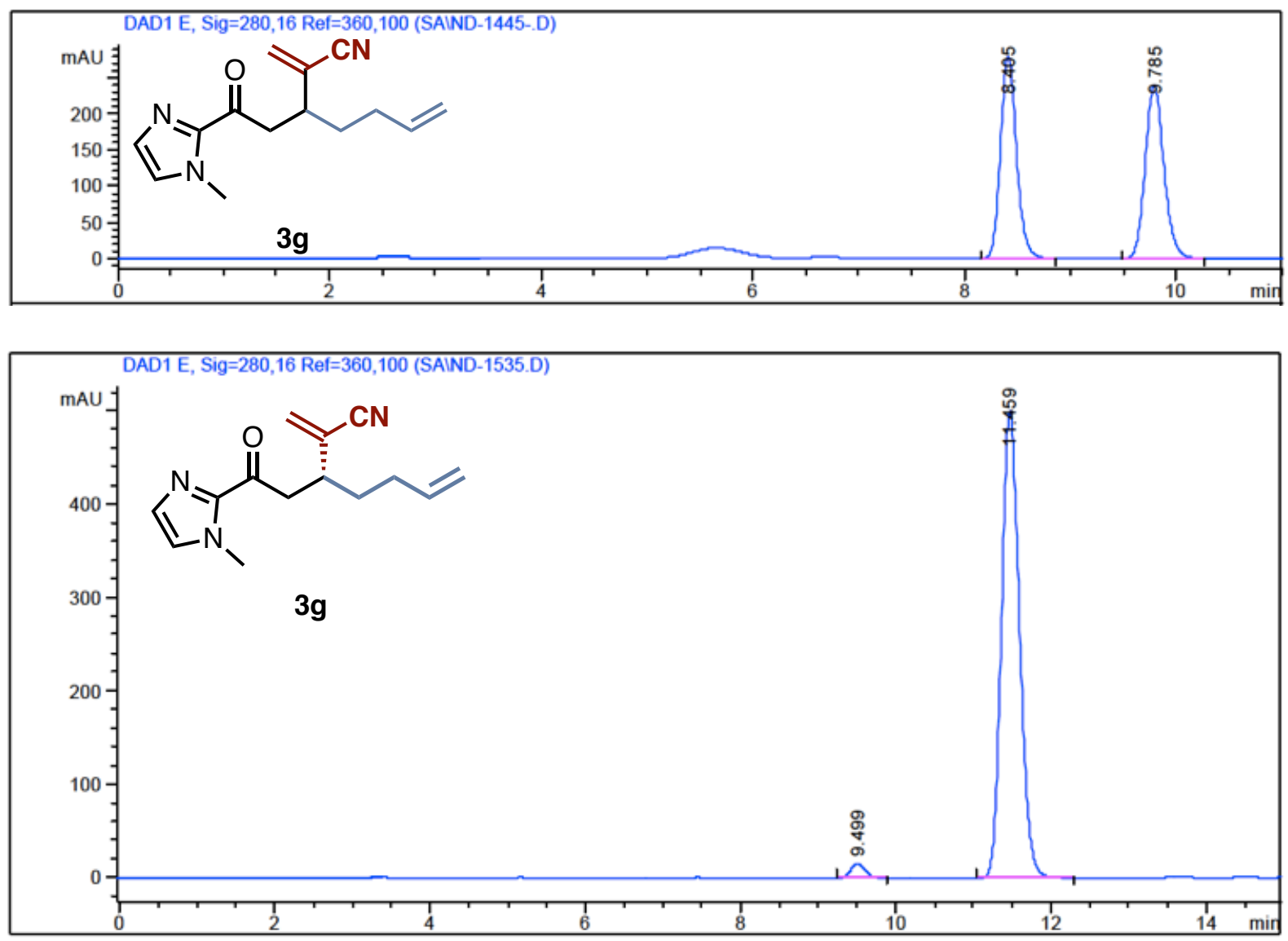

\begin{tabular}{|c|c|c|c|c|c|c|}
\hline $\begin{array}{c}\text { Peak } \\
\#\end{array}$ & $\begin{array}{c}\text { RetTime } \\
\text { [min] }\end{array}$ & Type & $\begin{array}{l}\text { Width } \\
\text { [min] }\end{array}$ & $\begin{array}{c}\text { Area } \\
{\left[m A U^{*} s\right]}\end{array}$ & $\begin{array}{l}\text { Height } \\
\text { [mAU] }\end{array}$ & $\begin{array}{c}\text { Area } \\
\&\end{array}$ \\
\hline & & & & 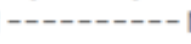 & & \\
\hline & & BV & & 202.66545 & & 631 \\
\hline 2 & 11.459 & VB & 90 & 8025.26709 & 5209 & 97.5369 \\
\hline
\end{tabular}




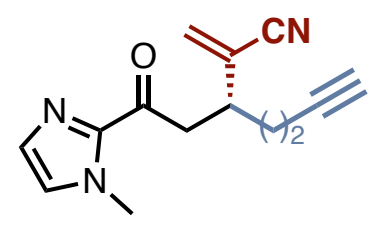

Molecular formula: $\mathrm{C}_{14} \mathrm{H}_{15} \mathrm{~N}_{3} \mathrm{O}$

$\mathbf{M W}=241.29 \mathrm{~g} \cdot \mathrm{mol}^{-1}$

The title compound was isolated as an amorphous solid (29 mg, $0.124 \mathrm{mmol}, 46 \%, 93 \%$ ee $)$ using general procedure F. Purified by flash column chromatography over silica gel $(\mathrm{Hex} / \mathrm{EtOAc}=7: 3)$.

${ }^{1} \mathrm{H}$ NMR (400 MHz, CDCl $)_{3} \delta 7.14(\mathrm{~s}, 1 \mathrm{H}), 7.03(\mathrm{~s}, 1 \mathrm{H}), 5.93(\mathrm{~s}, 1 \mathrm{H}), 5.92(\mathrm{~s}, 1 \mathrm{H}), 3.98(\mathrm{~s}, 3 \mathrm{H})$, $3.45(\mathrm{dd}, J=17.1,8.5 \mathrm{~Hz}, 1 \mathrm{H}), 3.33-3.20(\mathrm{~m}, 2 \mathrm{H}), 2.31$ (dtd, $J=17.1,6.2,2.6 \mathrm{~Hz}, 1 \mathrm{H}), 2.17$ (dtd, $J=17.1,7.8,2.6 \mathrm{~Hz}, 1 \mathrm{H}), 1.98(\mathrm{t}, J=2.7 \mathrm{~Hz}, 1 \mathrm{H}), 1.80(\mathrm{t}, J=9.7,7.1 \mathrm{~Hz}, 2 \mathrm{H})$.

${ }^{13} \mathrm{C}$ NMR $\left(101 \mathrm{MHz}, \mathrm{CDCl}_{3}\right) \delta 189.6,132.2,129.3,125.0,117.1,82.1,69.6,42.3,39.0,36.2$, $31.5,16.0$.

HRMS (ESI) m/z: [M+H] $]^{+}$calcd for $\mathrm{C}_{14} \mathrm{H}_{16} \mathrm{~N}_{3} \mathrm{O}$ 242.1293; found: 242.1276 .

IR $\left(\mathrm{cm}^{-1}\right): 3295,2931,2857,2222,1678,1411,1262$

$[a]_{D}^{20}=-21.9\left(c 0.97, \mathrm{CH}_{2} \mathrm{Cl}_{2}\right)$

HPLC analysis: carried out using a mixture of hexane and isopropyl alcohol (85:15 isocratic) at $1 \mathrm{~mL} \cdot \mathrm{min}^{-1}$, at $30{ }^{\circ} \mathrm{C}$ on an IC column, with UV detection $(\lambda=280 \mathrm{~nm})$. Retention times: 17.15 and $19.83 \mathrm{~min}$. 
${ }^{1} \mathrm{H}-\mathrm{NMR}$ of compound $3 \mathrm{~h}\left(400 \mathrm{MHz}, \mathrm{CDCl}_{3}\right)$

商

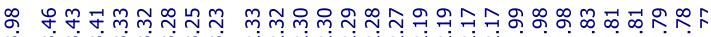

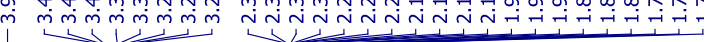<smiles>C#CCC(CC(=O)c1nccn1C)C(=C)C#N</smiles>

3h<smiles>C=CC=C</smiles>
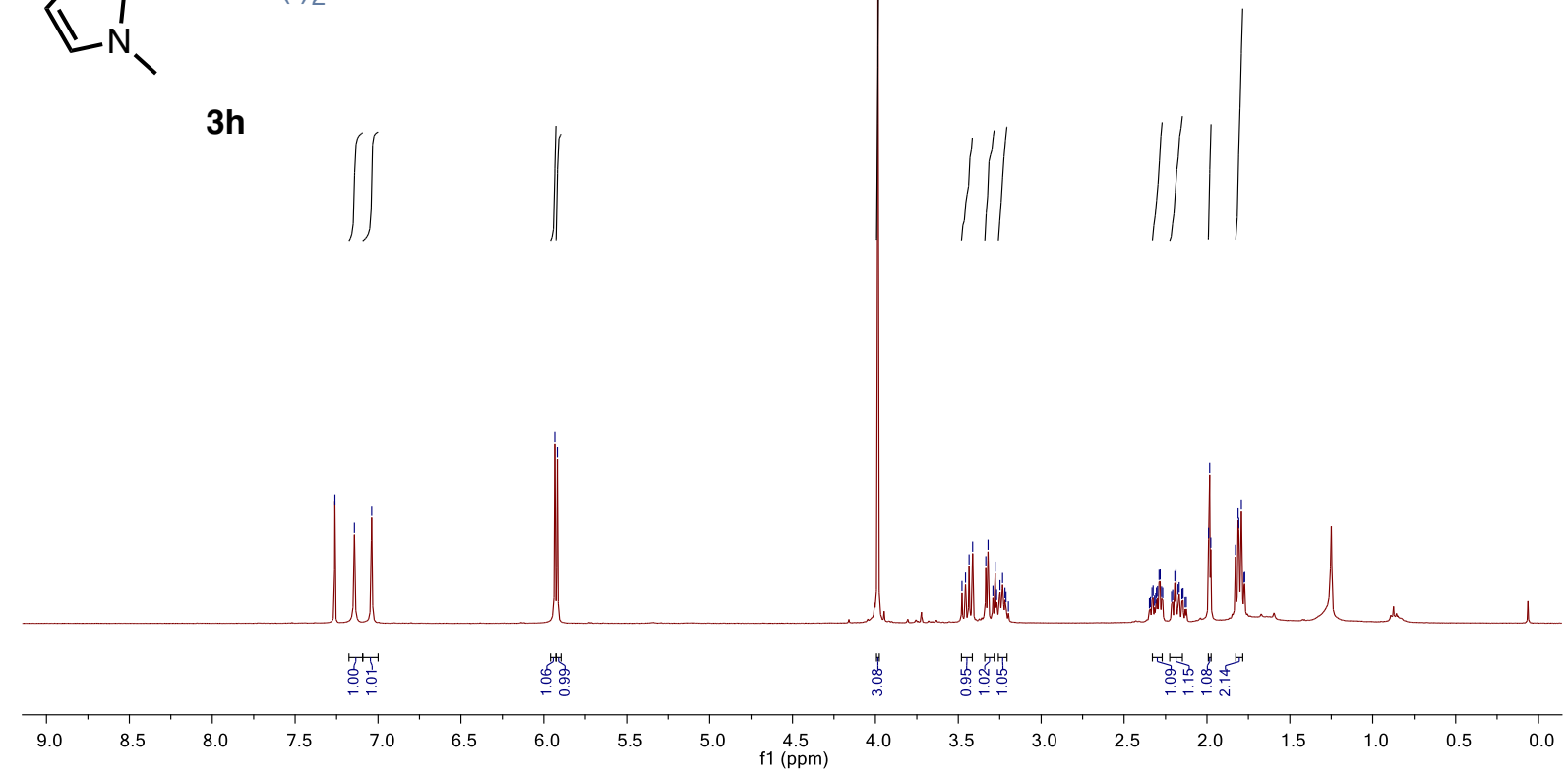

${ }^{13} \mathrm{C}-\mathrm{NMR}$ of compound $3 \mathrm{~h}\left(101 \mathrm{MHz}^{\mathrm{CDCl}} \mathrm{CD}_{3}\right)$

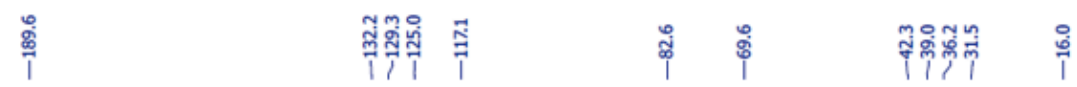

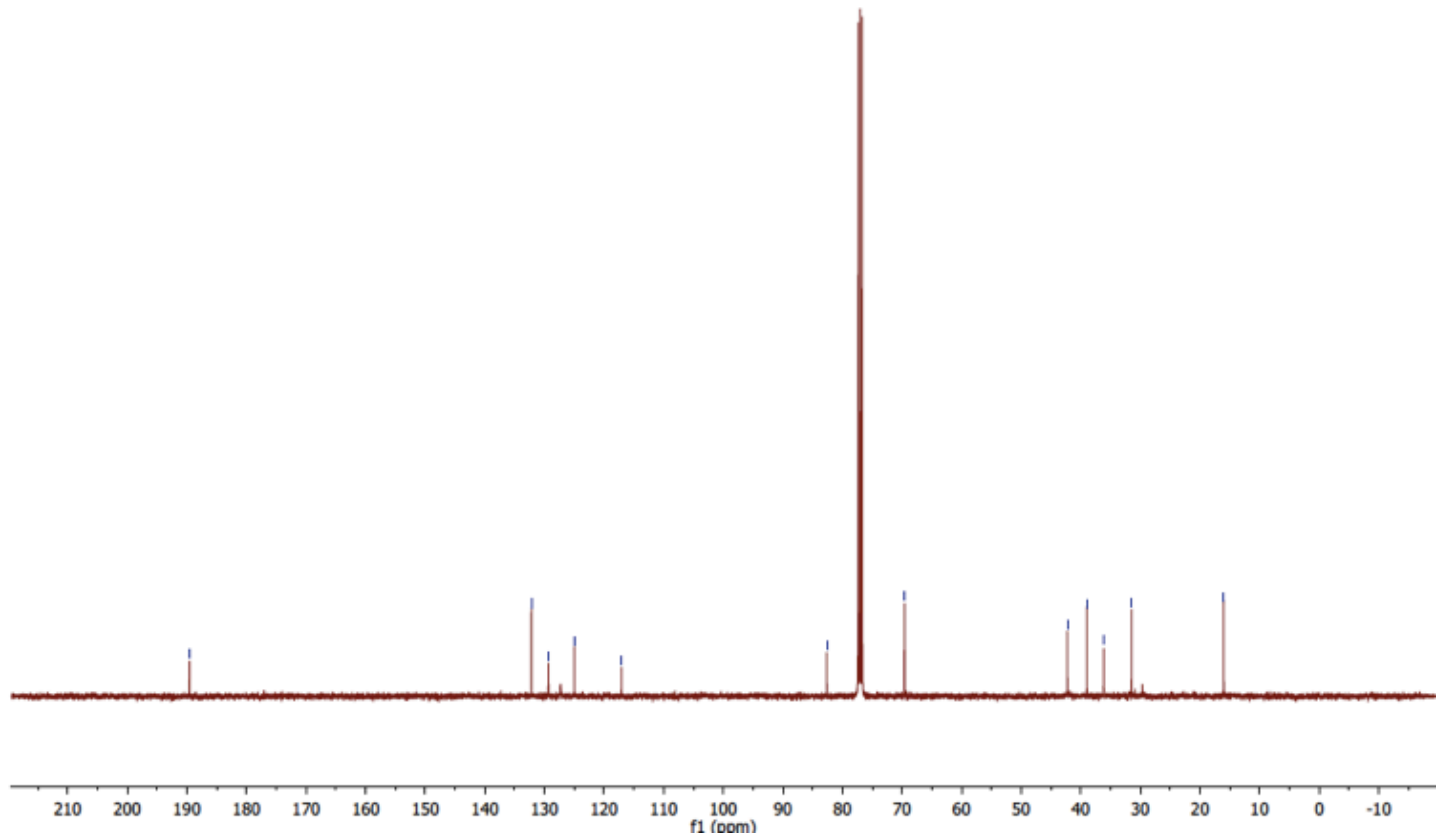



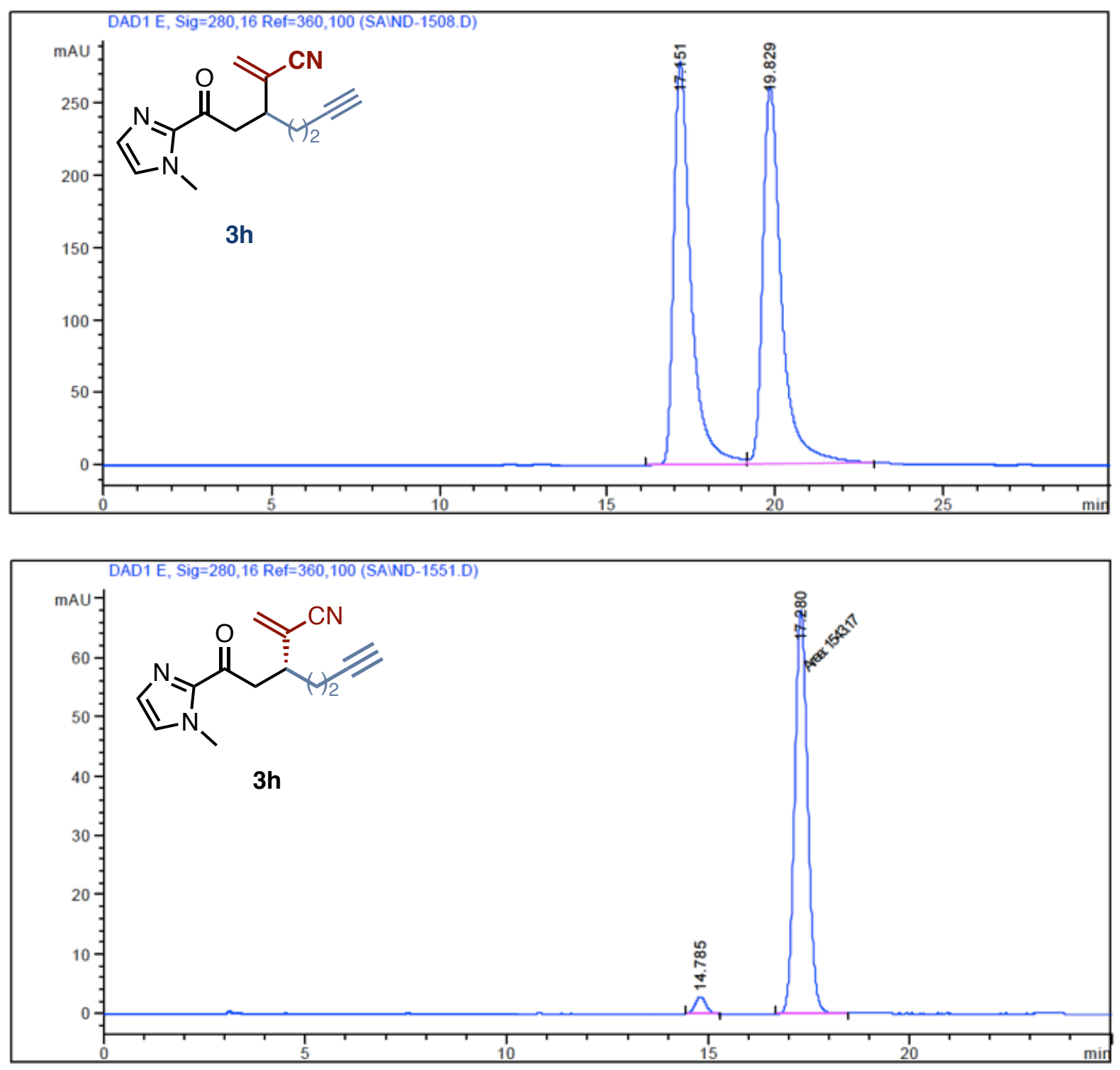

\begin{tabular}{|c|c|c|c|c|c|c|}
\hline $\begin{array}{c}\text { Peak } \\
\quad \#\end{array}$ & $\begin{array}{c}\text { RetTime } \\
\text { [min] }\end{array}$ & Type & $\begin{array}{l}\text { Width } \\
\text { [min] }\end{array}$ & $\begin{array}{c}\text { Area } \\
{\left[m A U^{*} s\right]}\end{array}$ & $\begin{array}{l}\text { Height } \\
\text { [mAU] }\end{array}$ & $\begin{array}{c}\text { Area } \\
\&\end{array}$ \\
\hline 1 & 14.785 & BB & 0.2943 & 53.29776 & 2.82210 & 3.3385 \\
\hline 2 & 17.280 & MM & 0.3781 & 1543.17297 & 68.03058 & 96.6615 \\
\hline
\end{tabular}




\section{3-[2-(1-Methyl-1H-imidazol-2-yl)-2-oxoethyl]-2-methylene-6-((triisopropylsilyl)oxy)}

hexanenitrile (3i)

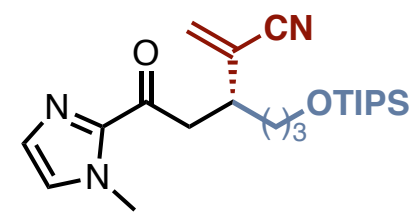

Molecular formula: $\mathrm{C}_{22} \mathrm{H}_{37} \mathrm{~N}_{3} \mathrm{O}_{2} \mathrm{Si}$

$\mathrm{MW}=403.64 \mathrm{~g} \cdot \mathrm{mol}^{-1}$

The title compound was isolated as an amorphous solid (61 $\mathrm{mg}, 0.151 \mathrm{mmol}, 56 \%, 93 \%$ ee $)$ using general procedure F. Purified by flash column chromatography over silica gel $\left(\mathrm{Hex} / \mathrm{Et}_{2} \mathrm{O}=3: 7\right)$.

${ }^{1}$ H NMR $\left(400 \mathrm{MHz}_{\mathrm{C}} \mathrm{CDCl}_{3}\right) \delta 7.14(\mathrm{~d}, J=0.9 \mathrm{~Hz}, 1 \mathrm{H}), 7.03(\mathrm{~d}, 0.9 \mathrm{~Hz}, 1 \mathrm{H}), 5.88(\mathrm{~s}, 1 \mathrm{H}), 5.82$ (s, 1H), $3.98(\mathrm{~s}, 3 \mathrm{H}), 3.69(\mathrm{t}, J=6.2 \mathrm{~Hz}, 2 \mathrm{H}), 3.41(\mathrm{dd}, J=17.1,8.6 \mathrm{~Hz}, 1 \mathrm{H}), 3.29(\mathrm{dd}, J=17.2$, $5.5 \mathrm{~Hz}, 1 \mathrm{H}), 3.02(\mathrm{tt}, J=8.7,5.6 \mathrm{~Hz}, 2 \mathrm{H}), 1.72-1.48(\mathrm{~m}, 6 \mathrm{H}), 1.05-1.03(\mathrm{~m}, 18 \mathrm{H})$.

${ }^{13} \mathrm{C}$ NMR $\left(101 \mathrm{MHz}, \mathrm{CDCl}_{3}\right) \delta 190.0,142.8,131.1,129.3,127.2,126.3,117.4,62.8,42.8$, $40.2,36.1,30.3,29.7,18.0,12.0$.

HRMS (ESI) m/z: [M+Na] ${ }^{+}$calcd for $\mathrm{C}_{22} \mathrm{H}_{37} \mathrm{~N}_{3} \mathrm{O}_{2} \mathrm{SiNa}$ 426.2552; found: 426.2533 .

IR $\left(\mathrm{cm}^{-1}\right): 2944,2866,2224,1741,1678,1466,1411,1106,1073$.

$[\boldsymbol{a}]_{\boldsymbol{D}}^{\mathbf{2 0}}=-12.2\left(\right.$ c 2.13, $\left.\mathrm{CH}_{2} \mathrm{Cl}_{2}\right)$

HPLC analysis: carried out using a mixture of hexane and isopropyl alcohol (90:10 isocratic) at $1 \mathrm{~mL} \cdot \mathrm{min}^{-1}$, at $30{ }^{\circ} \mathrm{C}$ on an IC column, with UV detection $(\lambda=280 \mathrm{~nm})$. Retention times: 7.89 and $9.37 \mathrm{~min}$. 
${ }^{1} \mathrm{H}-\mathrm{NMR}$ of compound $3 \mathrm{i}\left(400 \mathrm{MHz}, \mathrm{CDCl}_{3}\right)$

䰹

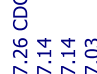<smiles>C=C(C#N)[C@H](CO[SnH3])CC(=O)c1nccn1C</smiles>

$3 \mathbf{i}$

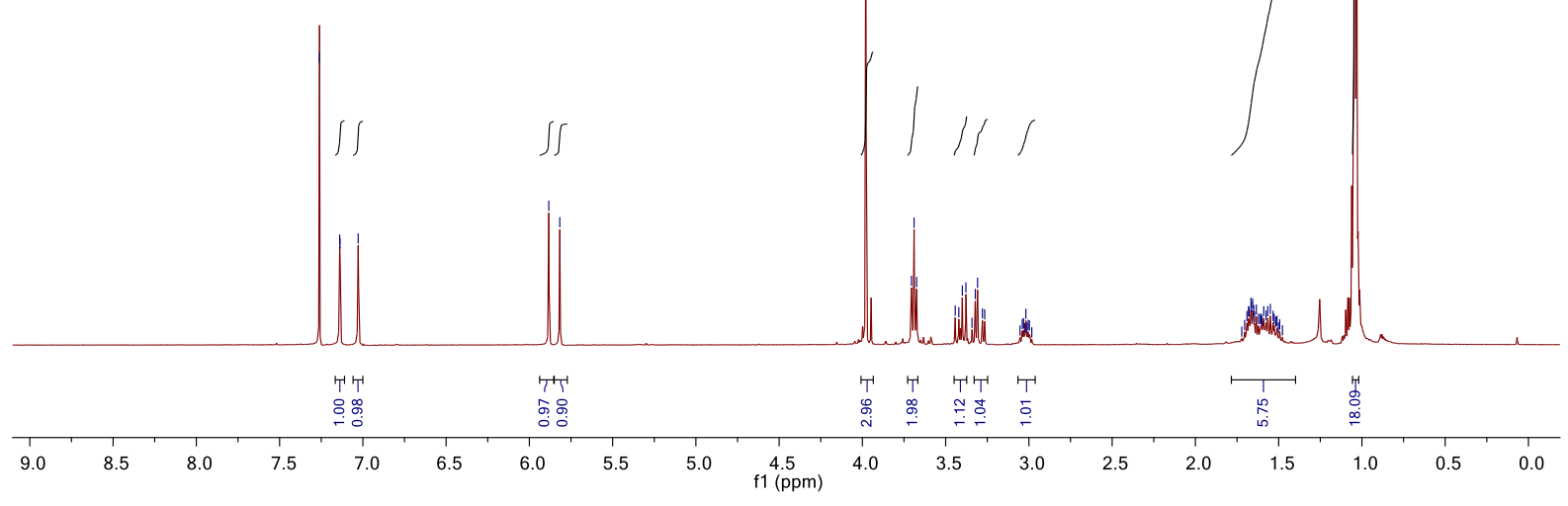

${ }^{13} \mathrm{C}-\mathrm{NMR}$ of compound $3 \mathrm{i}\left(101 \mathrm{MHz}, \mathrm{CDCl}_{3}\right)$

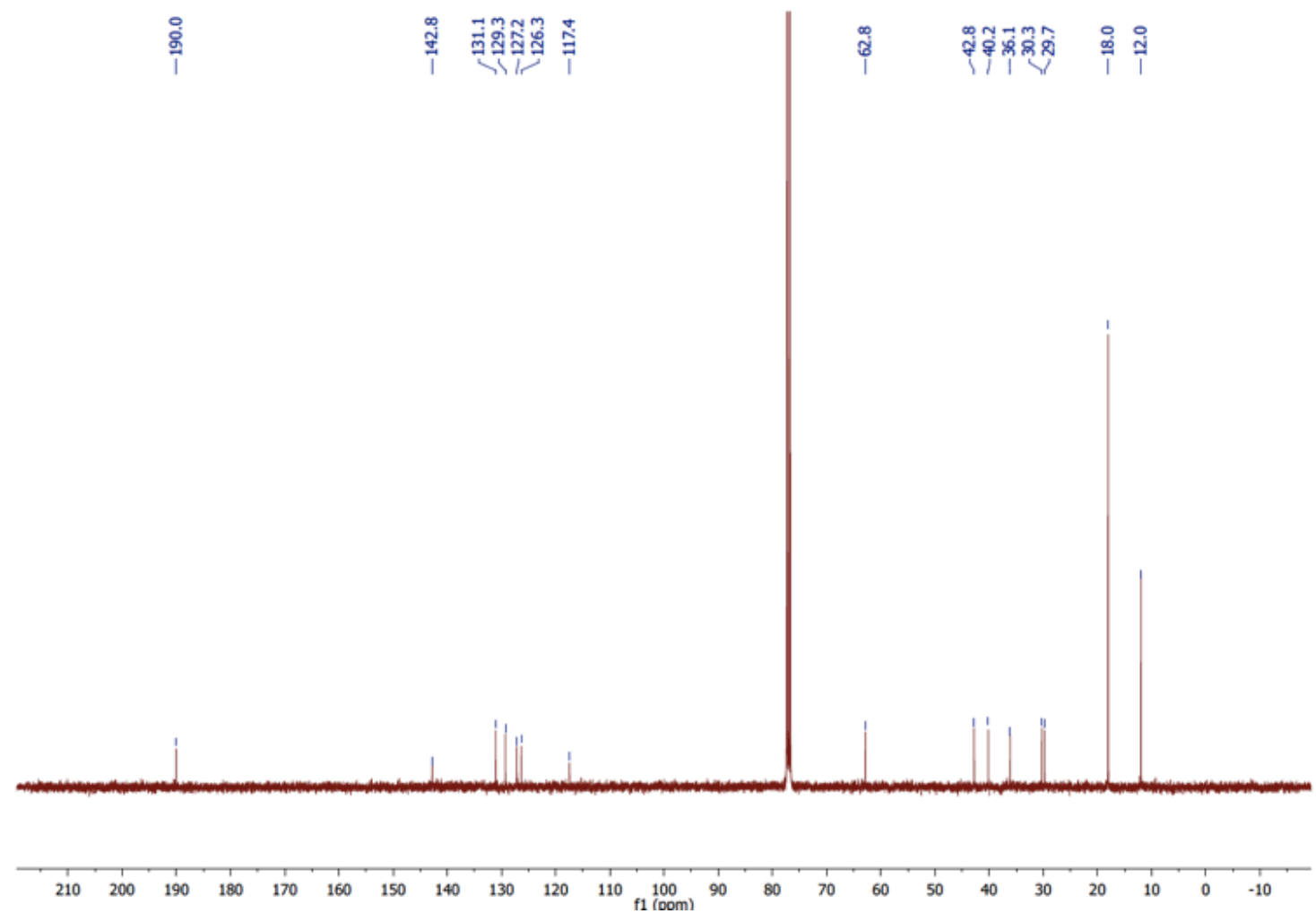



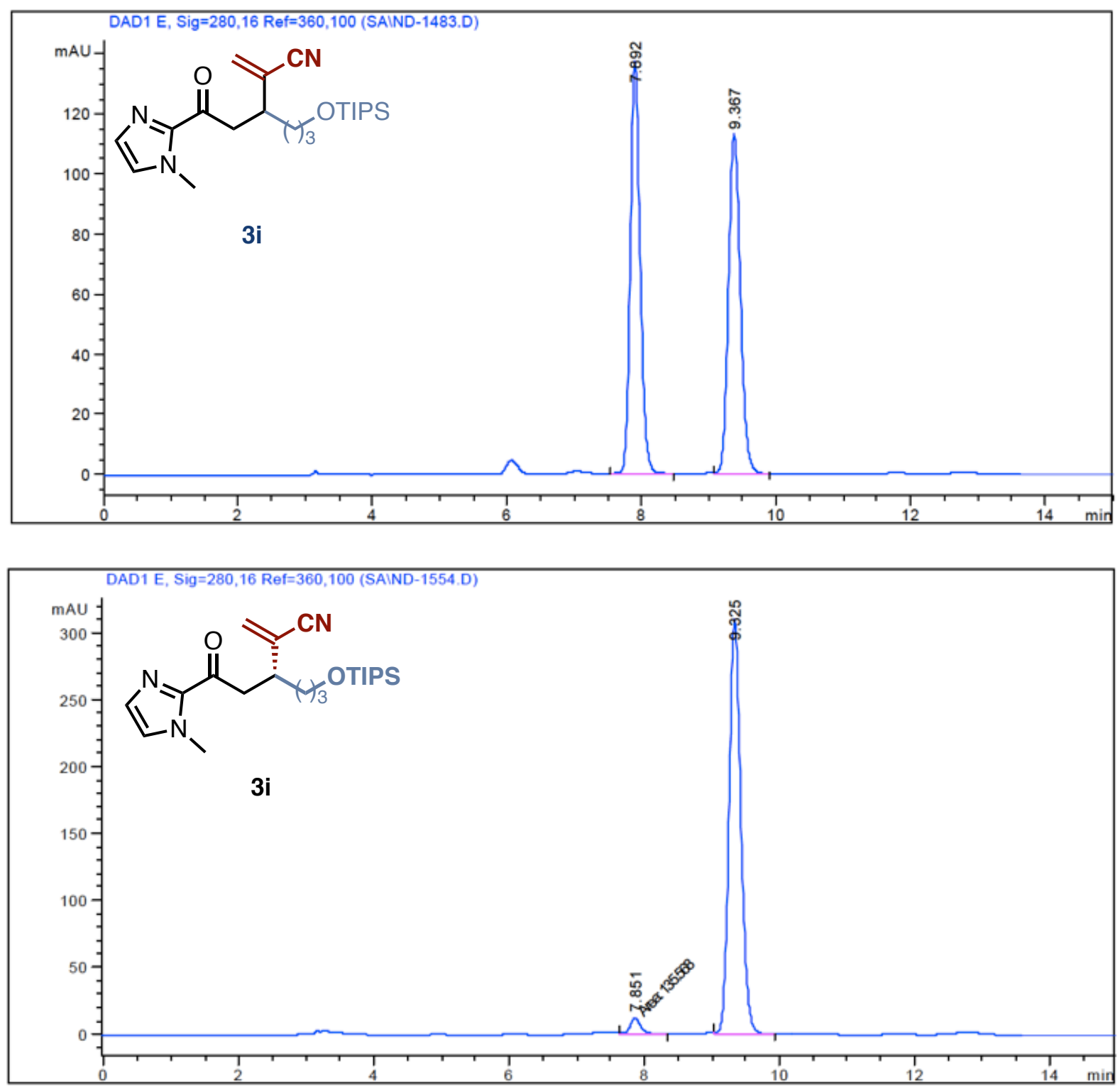

\begin{tabular}{|c|c|c|c|c|c|c|}
\hline $\begin{array}{c}\text { Peak } \\
\sharp\end{array}$ & $\begin{array}{c}\text { RetTime } \\
\text { [min] }\end{array}$ & Type & $\begin{array}{l}\text { Width } \\
\text { [min] }\end{array}$ & $\begin{array}{c}\text { Area } \\
{\left[\mathrm{mAU}^{*} \mathrm{~s}\right]}\end{array}$ & $\begin{array}{l}\text { Height } \\
\text { [mAU] }\end{array}$ & $\begin{array}{c}\text { Area } \\
\quad \&\end{array}$ \\
\hline 1 & 7.851 & MM & 0.1883 & 135.56781 & 11.99829 & 3.4405 \\
\hline 2 & 9.325 & VB & 0.1891 & 3804.82373 & 310.29163 & 96.5595 \\
\hline
\end{tabular}


3-[(Benzyloxy)methyl]-5-(1-methyl-1H-imidazol-2-yl)-2-methylene-5-oxopentanenitrile (3j)<smiles>C=C(C#N)[C@H](COc1ccccc1)CC(=O)c1nccn1C</smiles>

Molecular formula: $\mathrm{C}_{18} \mathrm{H}_{19} \mathrm{~N}_{3} \mathrm{O}$

$\mathbf{M W}=309.37 \mathrm{~g} \cdot \mathrm{mol}^{-1}$

The title compound was isolated as a clear oil (54 mg, $0.176 \mathrm{mmol}, 65 \%, 95 \%$ ee $)$ using general procedure F. Purified by flash column chromatography over silica gel (Hex/EtOAc/Acetone = 7:2:1).

${ }^{1}$ H NMR $\left(400 \mathrm{MHz}, \mathrm{CDCl}_{3}\right) \delta$ 7.33-7.26 (m, 5H), $7.13(\mathrm{~d}, J=0.9 \mathrm{~Hz}, 1 \mathrm{H}), 7.01(\mathrm{~d}, J=0.3 \mathrm{~Hz}$, 1H), $5.96(\mathrm{~s}, 1 \mathrm{H}), 5.92$ (d, $J=0.4 \mathrm{~Hz}, 1 \mathrm{H}), 4.50(\mathrm{~s}, 2 \mathrm{H}), 3.92$ (s, 3H), 3.61 (tdd, $J=13.1,7.0$, $3.7 \mathrm{~Hz}, 2 \mathrm{H}), 3.48(\mathrm{q}, J=7.0 \mathrm{~Hz}, 2 \mathrm{H}), 3.41-3.36(\mathrm{~m}, 2 \mathrm{H})$.

${ }^{13} \mathrm{C}$ NMR $\left(101 \mathrm{MHz}, \mathrm{CDCl}_{3}\right) \delta 189.5,142.7,137.8,132.2,129.3,128.3,127.7,127.6,127.2$, $123.9,117.6,73.3,71.2,65.9,40.2,39.3,36.1$.

HRMS (ESI) m/z: [M+H] calcd for $\mathrm{C}_{18} \mathrm{H}_{20} \mathrm{~N}_{3} \mathrm{O}$ 310.1555; found: 310.1551 .

IR $\left(\mathrm{cm}^{-1}\right):$ 2924, 2859, 2224, 1674, 1408, 1365, 1292, 1099.

$[\boldsymbol{a}]_{\boldsymbol{D}}^{20}=-7.5\left(\mathrm{c} 1.60, \mathrm{CH}_{2} \mathrm{Cl}_{2}\right)$

HPLC analysis: carried out using a mixture of hexane and isopropyl alcohol (80:20 isocratic) at $1 \mathrm{~mL} \cdot \mathrm{min}^{-1}$, at $30{ }^{\circ} \mathrm{C}$ on an IC column, with UV detection $(\lambda=280 \mathrm{~nm})$. Retention times: 15.52 and $17.83 \mathrm{~min}$. 


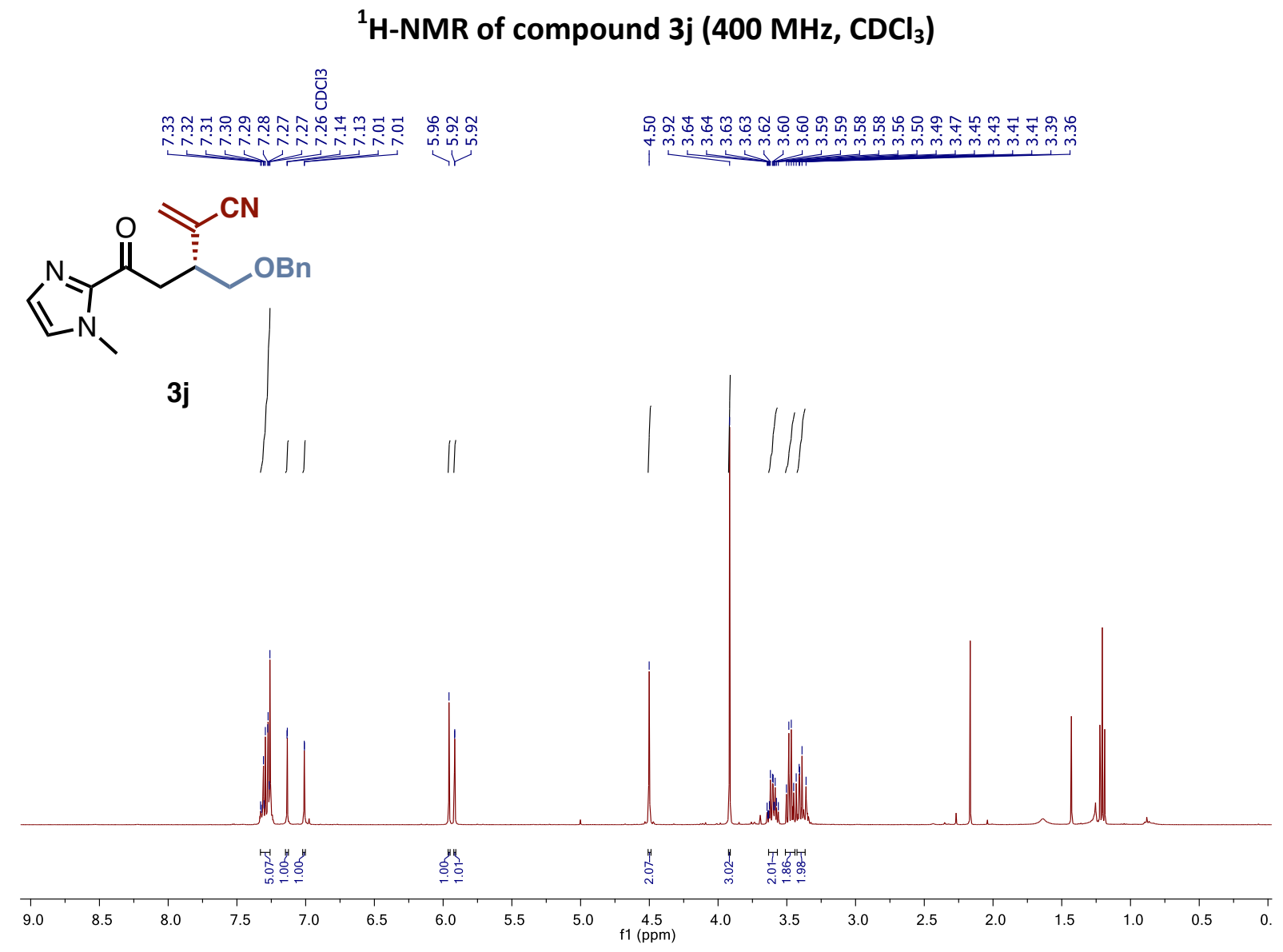

${ }^{13} \mathrm{C}-\mathrm{NMR}$ of compound 3j (101 MHz, $\mathrm{CDCl}_{3}$ )
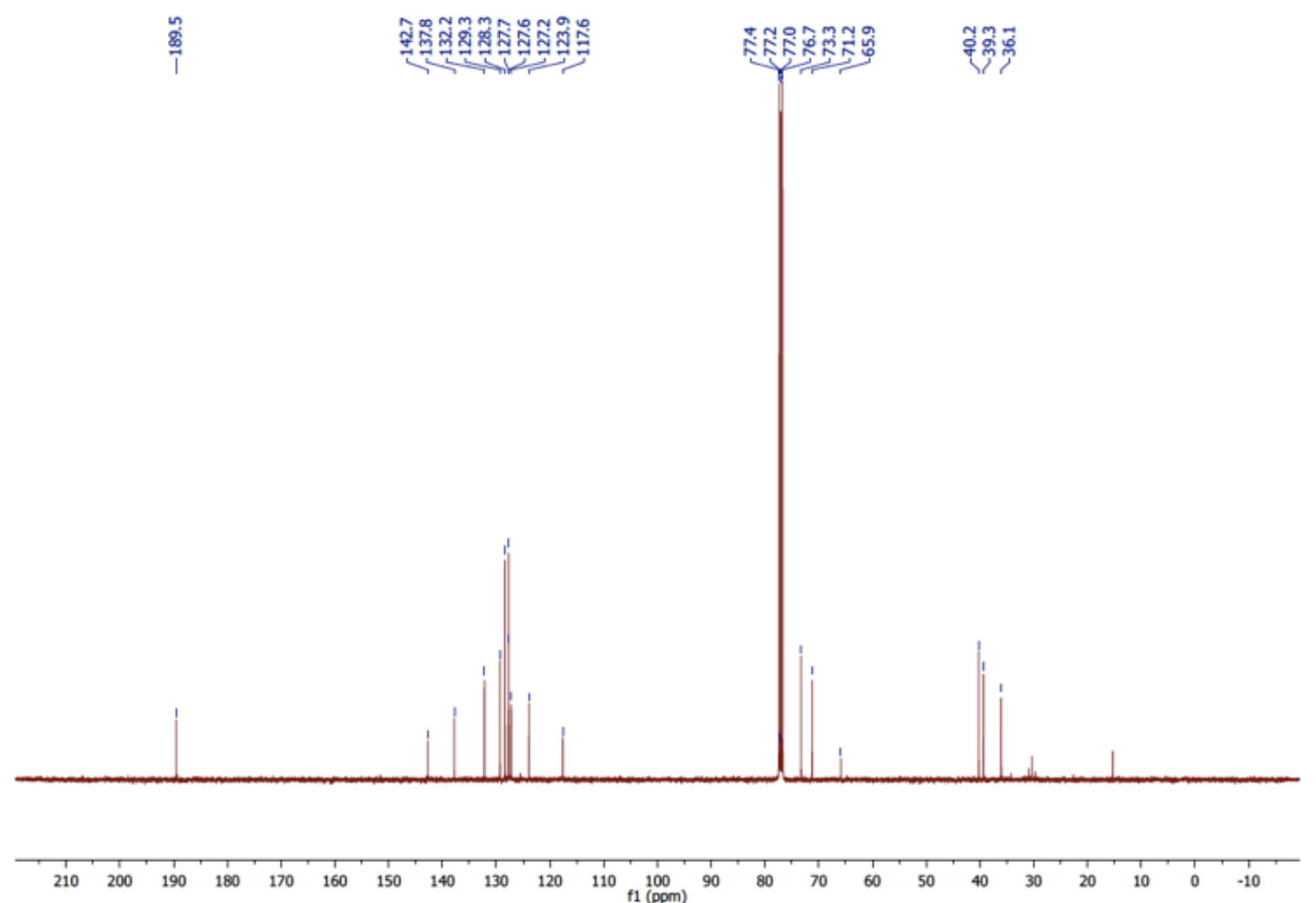

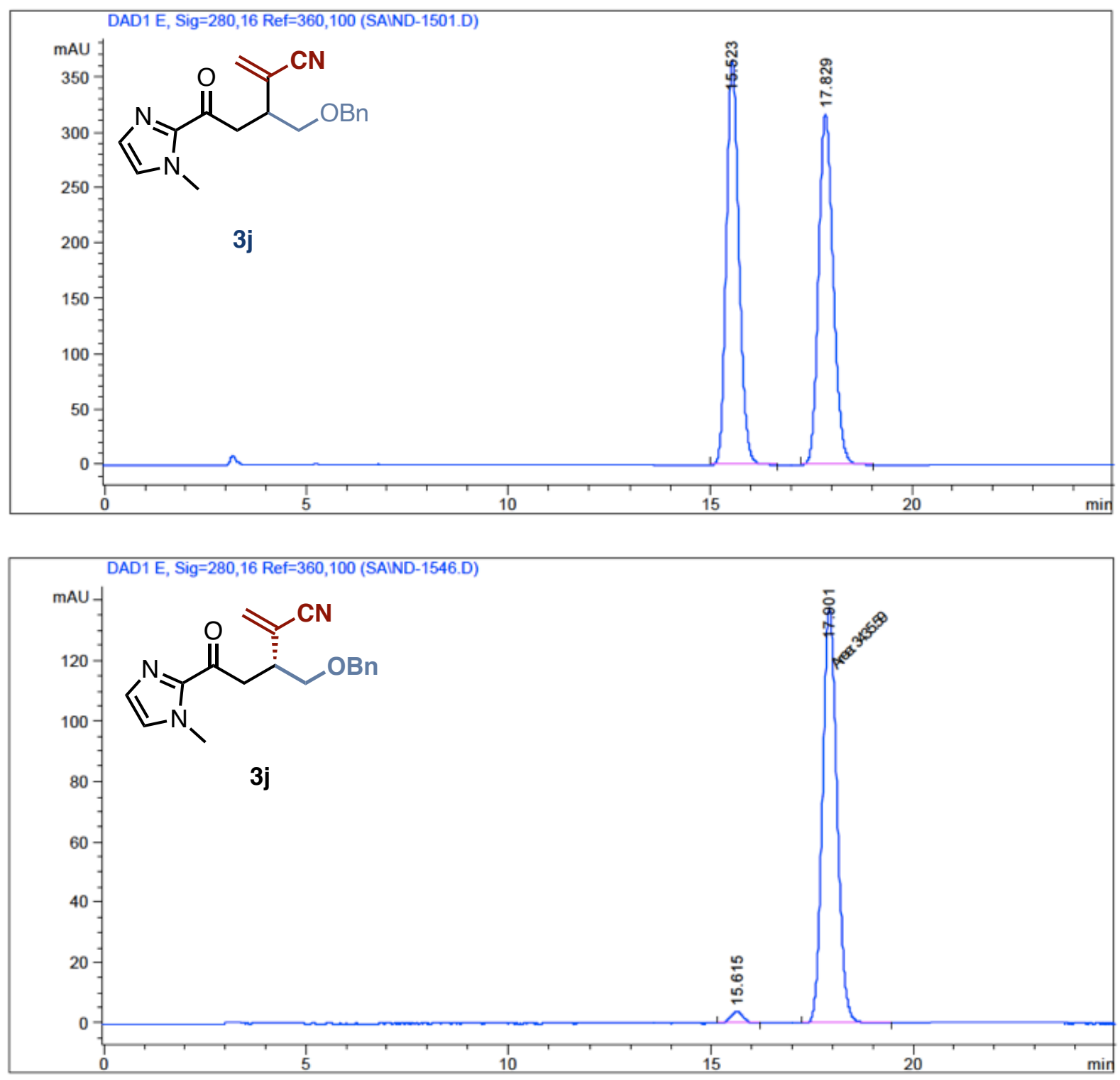

\begin{tabular}{|c|c|c|c|c|c|c|}
\hline $\begin{array}{c}\text { Peak } \\
+\end{array}$ & $\begin{array}{c}\text { RetTime } \\
\text { [min] }\end{array}$ & Type & $\begin{array}{l}\text { width } \\
\text { [min] }\end{array}$ & $\begin{array}{c}\text { Area } \\
\text { [mAU*s] }\end{array}$ & $\begin{array}{l}\text { Height } \\
\text { [mAU] }\end{array}$ & $\begin{array}{c}\text { Area } \\
\text { \& }\end{array}$ \\
\hline & & & & & & \\
\hline 1 & 15.615 & $\mathrm{BB}$ & 0.34 & 83.92729 & 3.85302 & 2.3846 \\
\hline 2 & 17.901 & $\mathrm{MM}$ & 0.4162 & 3435.58740 & 137.56583 & 97.6154 \\
\hline
\end{tabular}


oxopentanenitrile (3k)

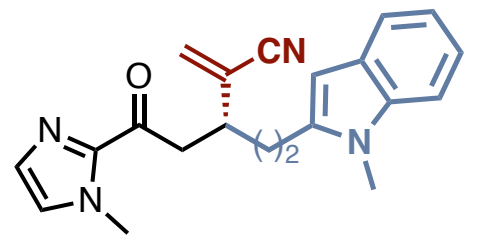

Molecular formula: $\mathrm{C}_{21} \mathrm{H}_{22} \mathrm{~N}_{4} \mathrm{O}$

$\mathrm{MW}=346.43 \mathrm{~g} \cdot \mathrm{mol}^{-1}$

The title compound was isolated as a clear oil ( $25 \mathrm{mg}, 0.073 \mathrm{mmol}, 27 \%, 88 \%$ ee $)$ using general procedure F. Purified by flash column chromatography over silica gel $($ Hex/EtOAc/Acetone $=7: 2: 1)$.

${ }^{1}$ H NMR $\left(400 \mathrm{MHz}, \mathrm{CDCl}_{3}\right) \delta 7.56(\mathrm{dt}, J=7.9,1.0 \mathrm{~Hz}, 1 \mathrm{H}), 7.27(\mathrm{~m}, 1 \mathrm{H}), 7.21(\mathrm{ddd}, J=8.2,6.9$, $1.2 \mathrm{~Hz}, 1 \mathrm{H}$ ), 7.14 (d, J=1.0 Hz, 1H), 7.09 (ddd, J=8.0, 6.9, $1.1 \mathrm{~Hz}, 1 \mathrm{H}$ ), $7.02(\mathrm{~d}, J=0.9 \mathrm{~Hz}$, 1H), $6.86(\mathrm{~d}, J=1.0 \mathrm{~Hz}, 1 \mathrm{H}), 5.93(\mathrm{~d}, J=0.6 \mathrm{~Hz}, 1 \mathrm{H}), 5.84(\mathrm{~d}, J=0.7 \mathrm{~Hz}, 1 \mathrm{H}), 3.96(\mathrm{~s}, 3 \mathrm{H}), 3.74$ (s, 3H), 3.45 (dd, $J=17.1,8.6 \mathrm{~Hz}, 1 \mathrm{H}$ ), 3.35 (dd, $J=17.1,5.6 \mathrm{~Hz}, 1 \mathrm{H}), 3.13(\mathrm{q}, J=7.8,6.0 \mathrm{~Hz}$, $1 \mathrm{H}), 2.82(\mathrm{~m}, 1 \mathrm{H}), 2.73(\mathrm{~m}, 1 \mathrm{H}), 2.00(\mathrm{~m}, 2 \mathrm{H})$.

${ }^{13} \mathrm{C}$ NMR $\left(101 \mathrm{MHz} \mathrm{CDCl}_{3}\right) \delta 190.0,142.8,137.0,131.4,129.3,127.7,127.2,126.2,121.5$, $118.9,118.6,117.5,113.7,109.1,42.7,40.0,36.1,33.8,32.6,30.3,22.4$.

HRMS (ESI) m/z: [M+H] $]^{+}$calcd for $\mathrm{C}_{21} \mathrm{H}_{23} \mathrm{~N}_{4} \mathrm{O}$ 347.1872; found: 347.1848 .

IR $\left(\mathrm{cm}^{-1}\right):$ 2928, 2857, 2223, 1676, 1475, 1411, 1292, 1134

$[\boldsymbol{a}]_{\boldsymbol{D}}^{\mathbf{2 0}}=-62.9\left(c 0.83, \mathrm{CH}_{2} \mathrm{Cl}_{2}\right)$

HPLC analysis: carried out using a mixture of hexane and isopropyl alcohol (80:20 isocratic) at $1 \mathrm{~mL} \cdot \mathrm{min}^{-1}$, at $30{ }^{\circ} \mathrm{C}$ on an IC column, with UV detection $(\lambda=280 \mathrm{~nm})$. Retention times: 21.76 and $24.09 \mathrm{~min}$. 
${ }^{1} \mathrm{H}-\mathrm{NMR}$ of compound $3 \mathrm{k}\left(400 \mathrm{MHz}, \mathrm{CDCl}_{3}\right)$

荅

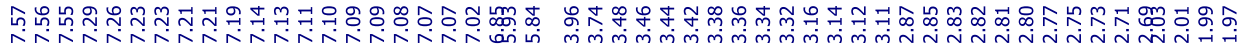

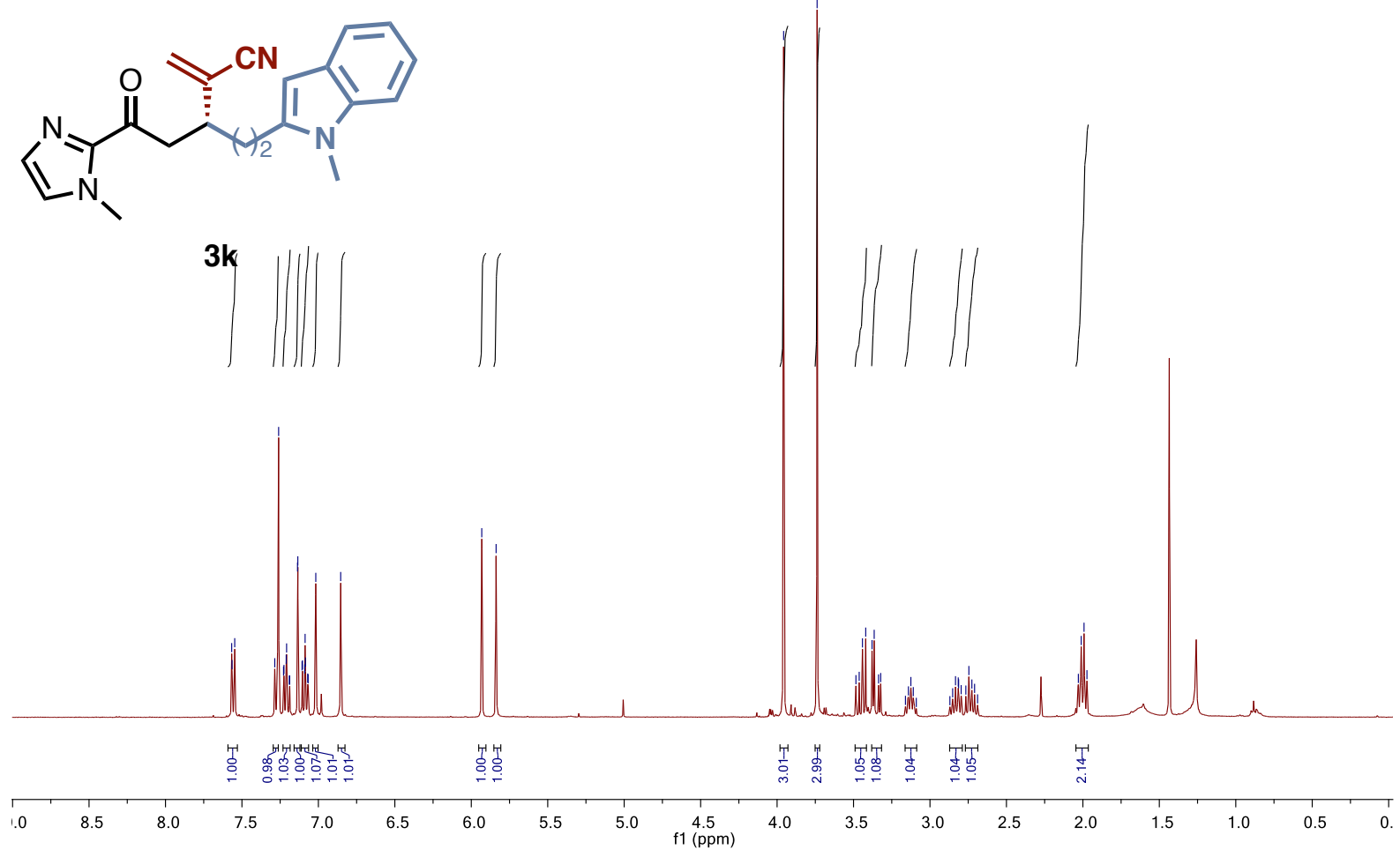

${ }^{13} \mathrm{C}-\mathrm{NMR}$ of compound 3k (101 MHz, $\mathrm{CDCl}_{3}$ )

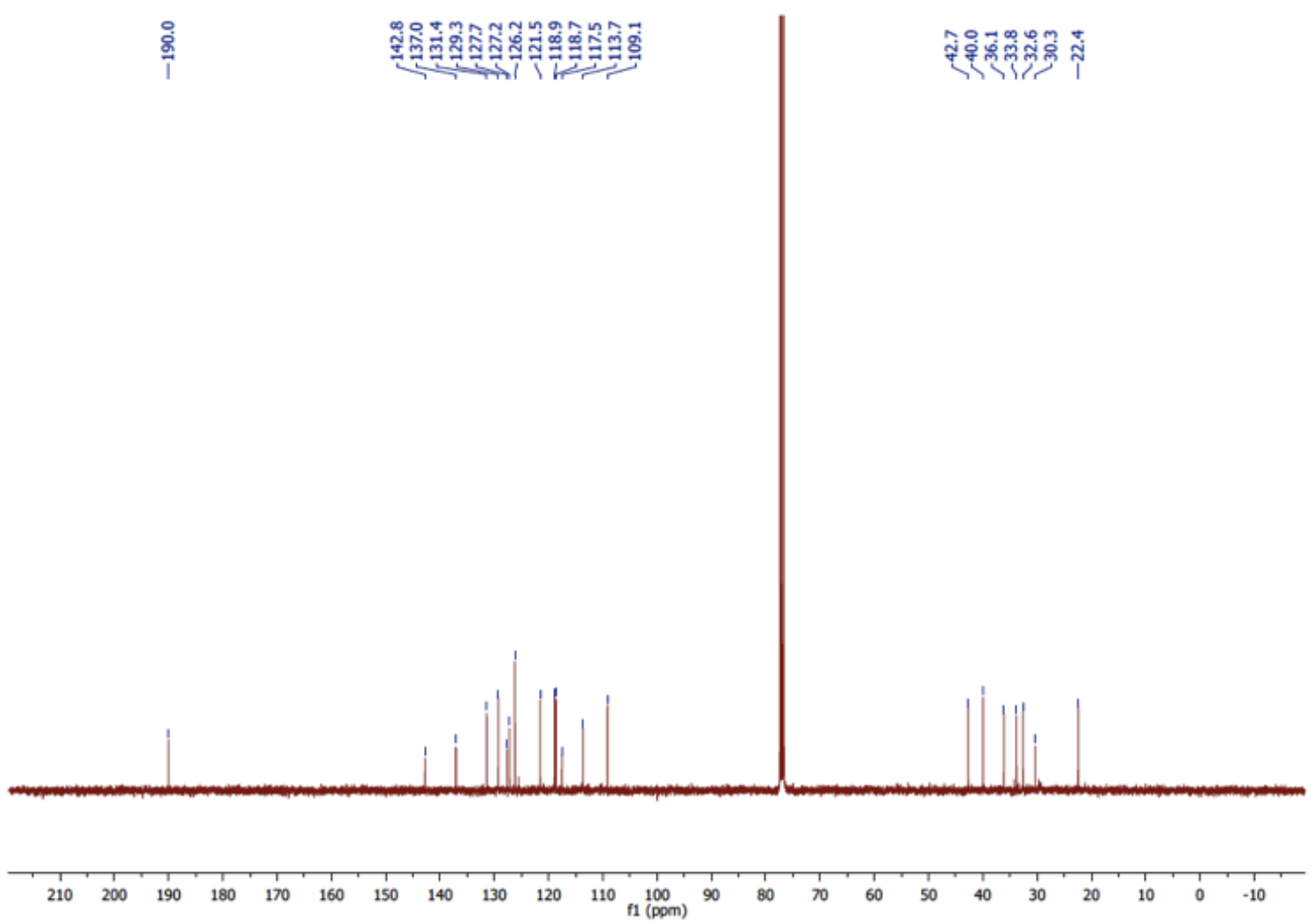



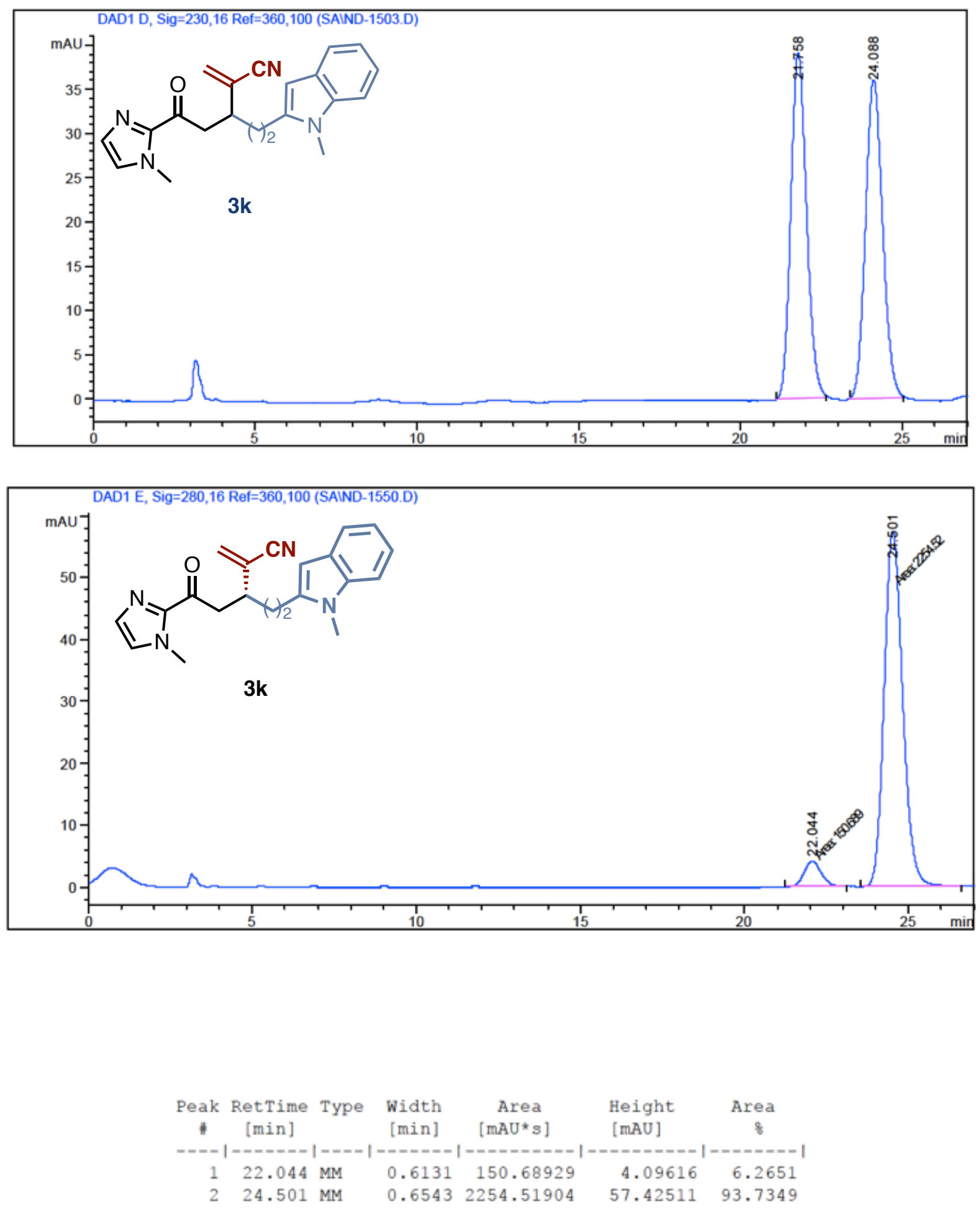


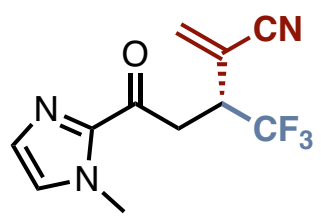

Molecular formula: $\mathrm{C}_{11} \mathrm{H}_{10} \mathrm{~F}_{3} \mathrm{~N}_{3} \mathrm{O}$

$\mathrm{MW}=257.22 \mathrm{~g} \cdot \mathrm{mol}^{-1}$

The title compound was isolated as a pale yellow solid (29 mg, $0.116 \mathrm{mmol}, 43 \%, 92 \%$ ee $)$ using general procedure F. Purified by flash column chromatography over silica gel $($ Hex/EtOAc/Acetone $=7: 2: 1)$.

${ }^{1} \mathrm{H}$ NMR $\left(400 \mathrm{MHz}, \mathrm{CDCl}_{3}\right) \delta 7.17(\mathrm{~d}, J=1.0 \mathrm{~Hz}, 1 \mathrm{H}), 7.08(\mathrm{~d}, J=0.9 \mathrm{~Hz}, 1 \mathrm{H}), 6.22(\mathrm{~s}, 1 \mathrm{H}), 6.16$ (s, 1H), $3.99(\mathrm{~s}, 3 \mathrm{H}), 3.86-3.77(\mathrm{~m}, 2 \mathrm{H}), 3.62(\mathrm{~m}, 1 \mathrm{H}), 3.30(\mathrm{dd}, J=17.2,5.7 \mathrm{~Hz}, 1 \mathrm{H}), 3.03$ $(\mathrm{tt}, J=8.4,5.7 \mathrm{~Hz}, 1 \mathrm{H}), 2.13(\mathrm{~m}, 1 \mathrm{H}), 2.04(\mathrm{~m}, 1 \mathrm{H}), 1.71-1.65(\mathrm{~m}, 2 \mathrm{H})$.

${ }^{13} \mathrm{C}$ NMR $\left(101 \mathrm{MHz}, \mathrm{CDCl}_{3}\right) \delta 186.5,142.0,137.1,129.8,127.7,126.7,123.9,117.2,117.2$, $116.3,43.5(q, J=28.9 \mathrm{~Hz}), 36.3(\mathrm{~d}, J=1.9 \mathrm{~Hz})$.

${ }^{19}$ F NMR $\left(377 \mathrm{MHz}, \mathrm{CDCl}_{3}\right) \delta-70.22(\mathrm{~d}, J=8.1 \mathrm{~Hz}, 3 \mathrm{~F})$.

HRMS: After several attempts, this compound was susceptible to insource fragmentation and was not thermostable to obtain accurate mass measurements

IR $\left(\mathrm{cm}^{-1}\right):$ 3116, 2959, 2229, 1682, 1415, 1359, 1261, 1194, 1164, 1119.

$[\boldsymbol{a}]_{D}^{20}=-15.6\left(c 0.67, \mathrm{CH}_{2} \mathrm{Cl}_{2}\right)$

HPLC analysis: carried out using a mixture of hexane and isopropyl alcohol (80:20 isocratic) at $1 \mathrm{~mL} \cdot \mathrm{min}^{-1}$, at $30{ }^{\circ} \mathrm{C}$ on an IC column, with UV detection $(\lambda=280 \mathrm{~nm})$. Retention times: 6.52 and $7.40 \mathrm{~min}$. 


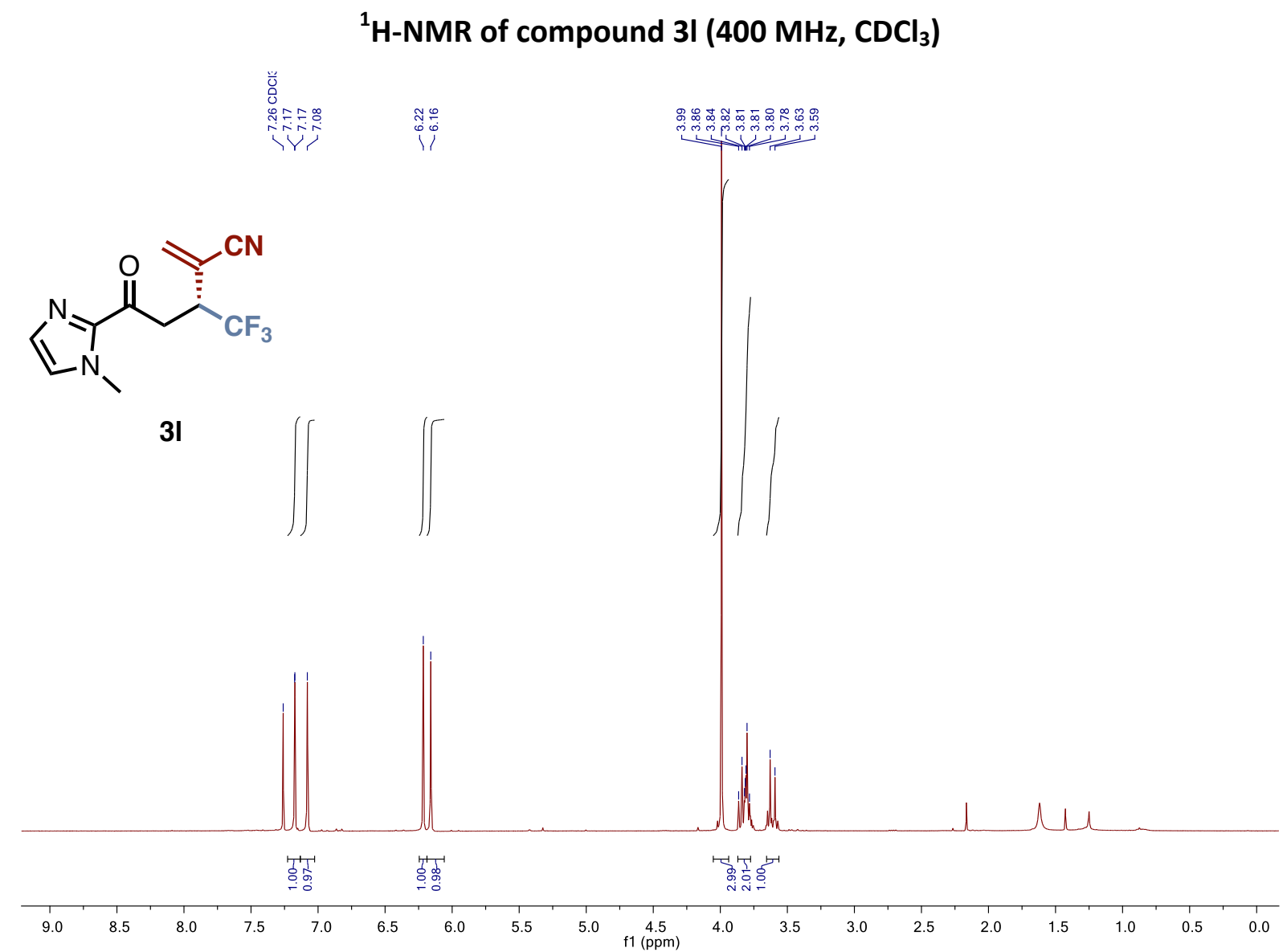

${ }^{13} \mathrm{C}-\mathrm{NMR}$ of compound $3 \mathrm{I}$ (101 MHz, $\mathrm{CDCl}_{3}$ )

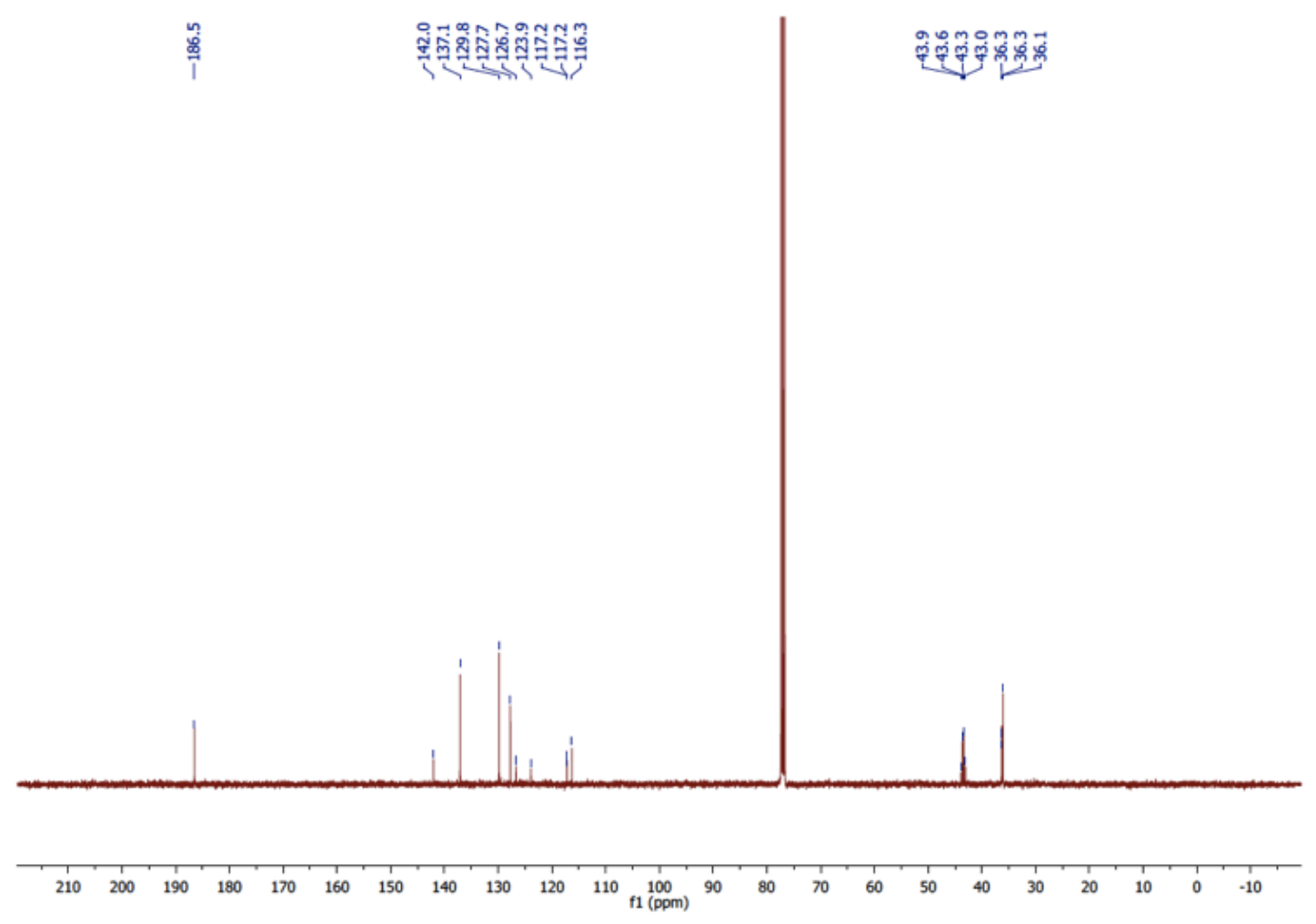


${ }^{19} \mathrm{~F}-\mathrm{NMR}$ of compound $3 \mathrm{l}\left(377 \mathrm{MHz}, \mathrm{CDCl}_{3}\right)$<smiles>C=C(C#N)[C@H](CC(=O)c1nccn1C)C(F)(F)F</smiles>

กู่

3I

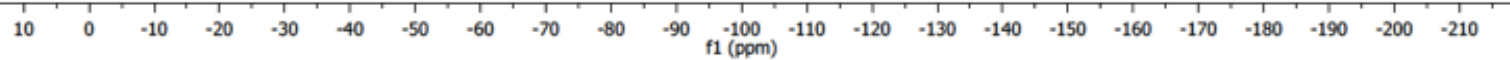



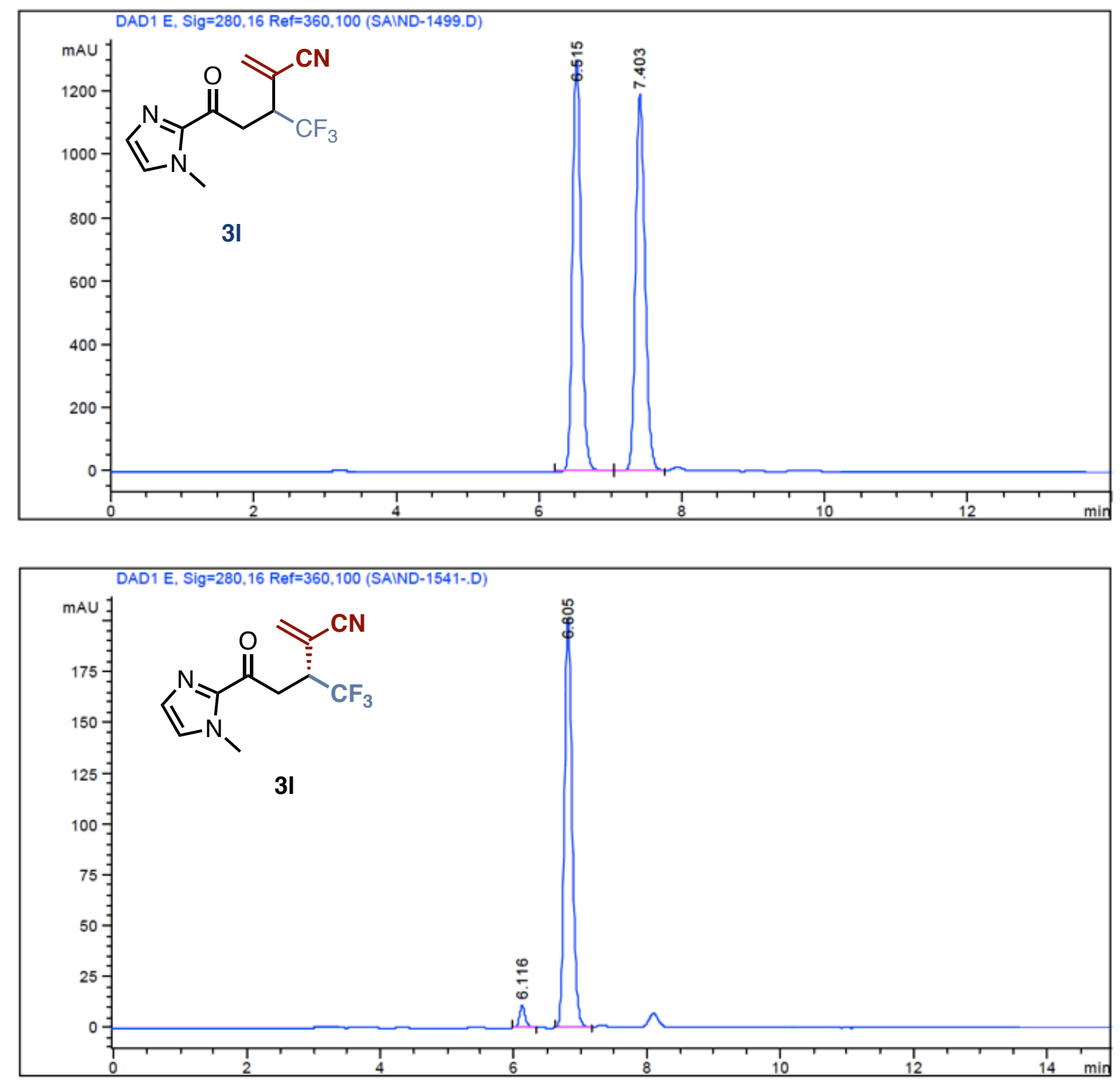

\begin{tabular}{|c|c|c|c|c|c|c|}
\hline $\begin{array}{c}\text { Peak } \\
\#\end{array}$ & $\begin{array}{c}\text { RetTime } \\
\text { [min] }\end{array}$ & Type & $\begin{array}{l}\text { Width } \\
\text { [min] }\end{array}$ & $\begin{array}{c}\text { Area } \\
{\left[\mathrm{mAU}^{\star} \mathrm{s}\right]}\end{array}$ & $\begin{array}{l}\text { Height } \\
\text { [mAU] }\end{array}$ & $\begin{array}{c}\text { Area } \\
8\end{array}$ \\
\hline \multicolumn{7}{|c|}{ 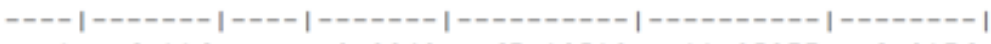 } \\
\hline 1 & & $P P_{1}$ & & 67.18510 & & \\
\hline & 6.805 & BV & 0.1253 & 1647.77271 & 201.66869 & 96.0824 \\
\hline
\end{tabular}




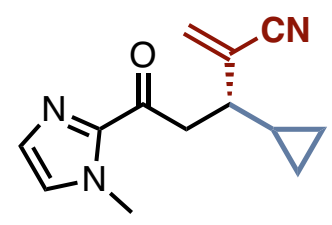

Molecular formula: $\mathrm{C}_{13} \mathrm{H}_{15} \mathrm{~N}_{3} \mathrm{O}$

$\mathrm{MW}=229.28 \mathrm{~g} \cdot \mathrm{mol}^{-1}$

The title compound was isolated as an amorphous solid (31 mg, $0.157 \mathrm{mmol}, 58 \%, 82 \%$ ee $)$ using general procedure F. Purified by flash column chromatography over silica gel $\left(\mathrm{Hex} / \mathrm{Et}_{2} \mathrm{O}=4: 6\right)$.

${ }^{1} \mathrm{H}$ NMR $\left(400 \mathrm{MHz}, \mathrm{CDCl}_{3}\right) \delta 7.13(\mathrm{~s}, 1 \mathrm{H}), 7.03(\mathrm{~s}, 1 \mathrm{H}), 5.85(\mathrm{~s}, 1 \mathrm{H}), 5.81(\mathrm{~s}, 1 \mathrm{H}), 3.97(\mathrm{~s}, 3 \mathrm{H})$, $3.55(\mathrm{dd}, J=16.7,8.6 \mathrm{~Hz}, 1 \mathrm{H}), 3.43(\mathrm{dd}, J=16.7,5.5 \mathrm{~Hz}, 1 \mathrm{H}), 2.22(\mathrm{~m}, 1 \mathrm{H}), 0.97(\mathrm{~m}, 1 \mathrm{H}), 0.63-$ $0.50(\mathrm{~m}, 2 \mathrm{H}), 0.30-0.18(\mathrm{~m}, 2 \mathrm{H})$.

${ }^{13} \mathrm{C}$ NMR $\left(101 \mathrm{MHz}^{\mathrm{C} C D C l}\right) \delta 190.2,143.0,130.1,129.4,127.3,126.5,118.2,45.3,42.8,36.3$, $15.4,5.2,4.8$.

HRMS (ESI) m/z: [M+H] $]^{+}$calcd for $\mathrm{C}_{13} \mathrm{H}_{16} \mathrm{~N}_{3} \mathrm{O}$ 230.1293; found: 230.1293.

IR $\left(\mathrm{cm}^{-1}\right):$ 3010, 2959, 1663, 1611, 1467, 1411, 1266, 1032

$[a]_{D}^{20}=-21.5\left(c=1.10, \mathrm{CH}_{2} \mathrm{Cl}_{2}\right)$

HPLC analysis: carried out using a mixture of hexane and isopropyl alcohol (70:30 isocratic) at $25{ }^{\circ} \mathrm{C}$ on an IC column, with UV detection $(\lambda=280 \mathrm{~nm})$. Retention times: 8.6 and $10.0 \mathrm{~min}$. 


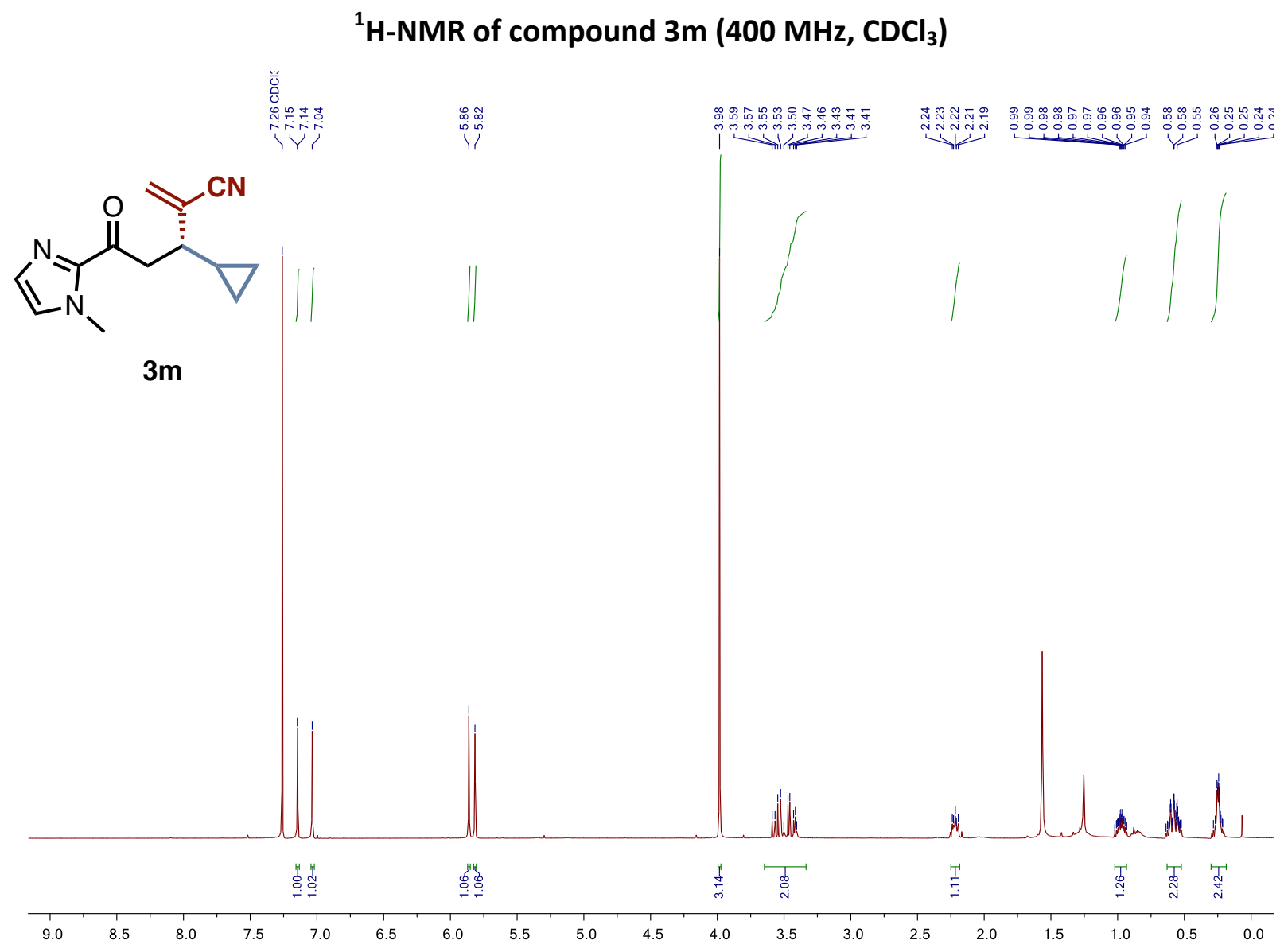

${ }^{13} \mathrm{C}-\mathrm{NMR}$ of compound $3 \mathrm{~m}\left(101 \mathrm{MHz}, \mathrm{CDCl}_{3}\right)$

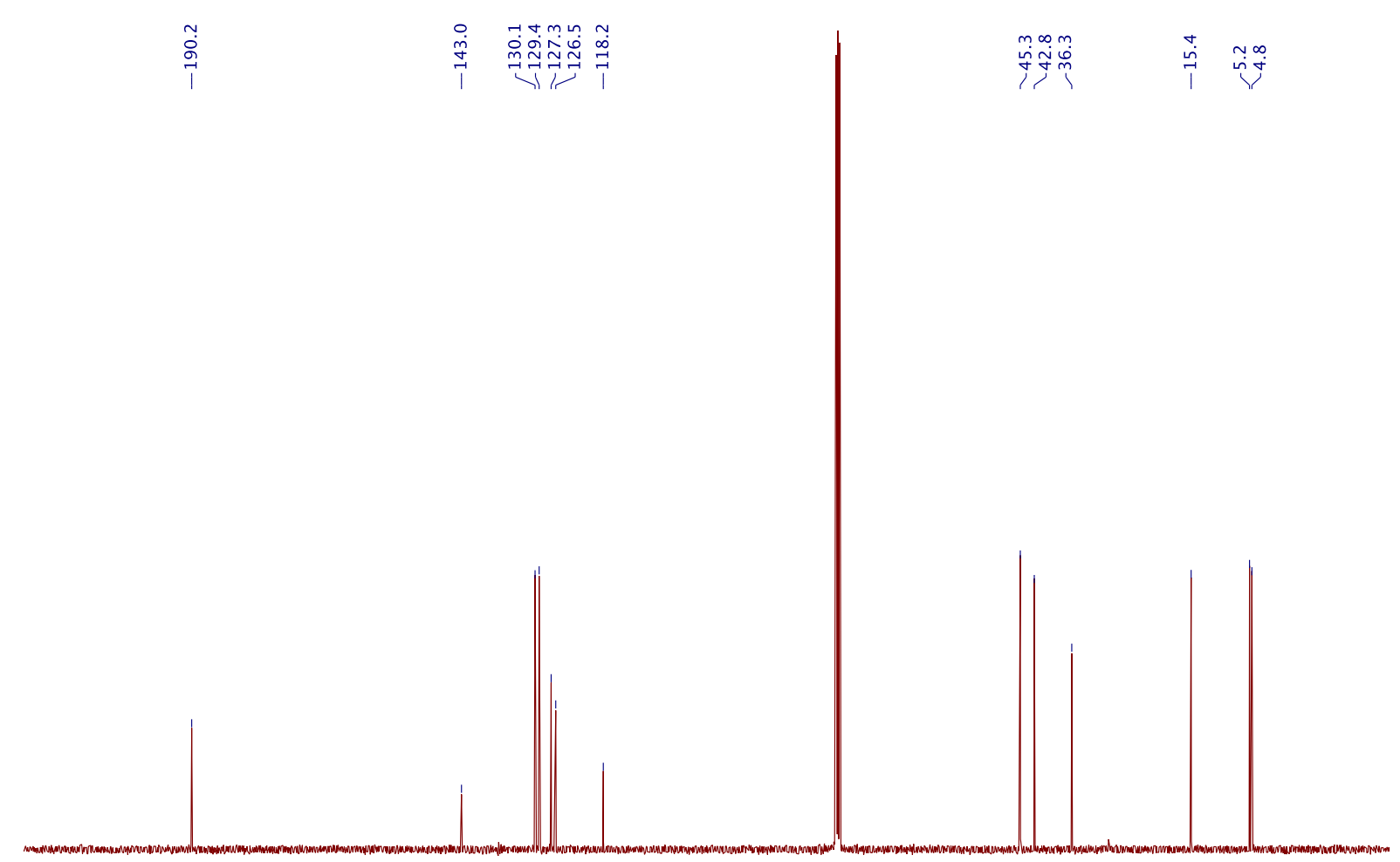

$\begin{array}{lllllllllllllllllllllll}210 & 200 & 190 & 180 & 170 & 160 & 150 & 140 & 130 & 120 & 110 & 100 & 90 & 80 & 70 & 60 & 50 & 40 & 30 & 20 & 10 & 0 & -10\end{array}$ 

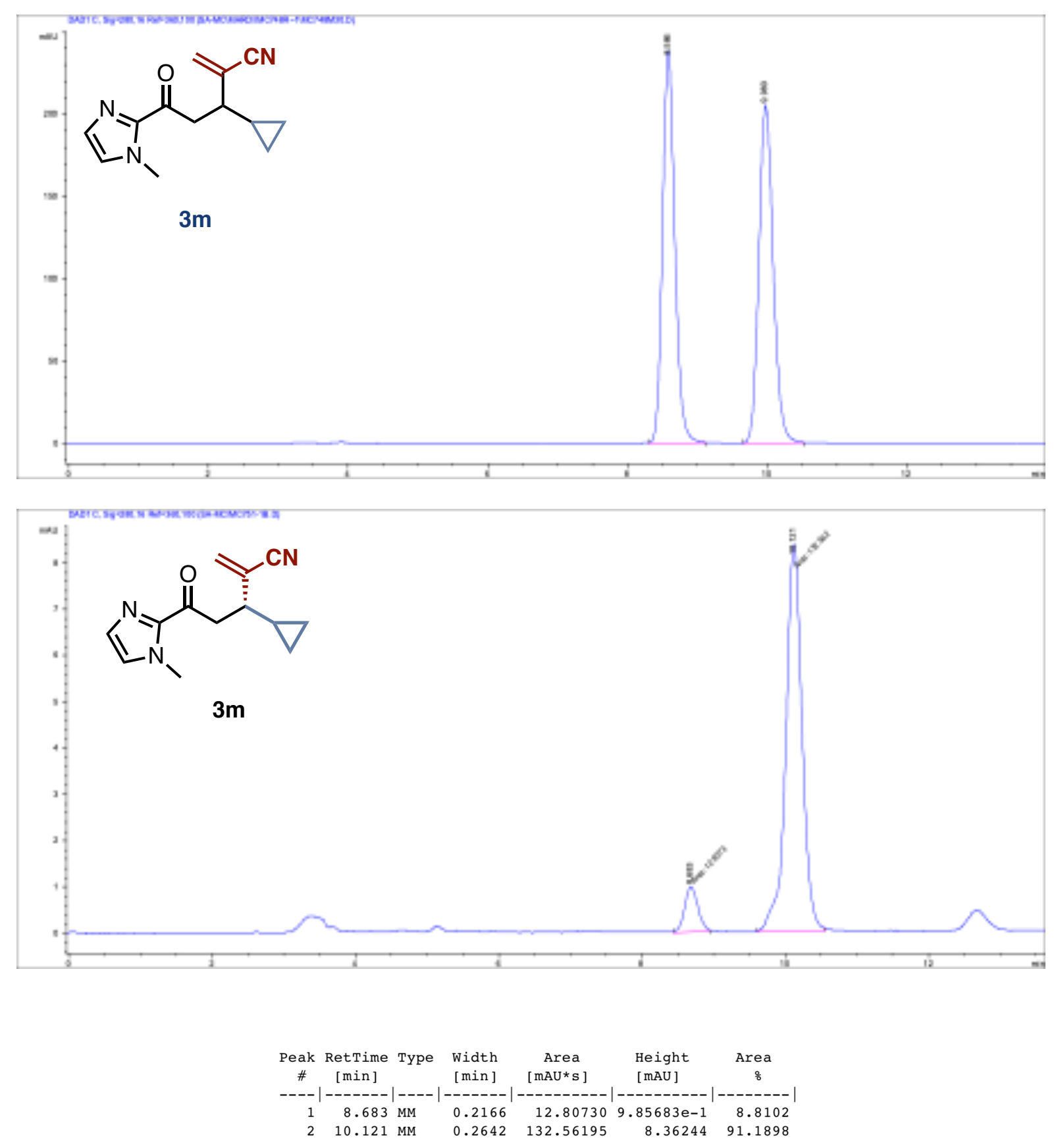


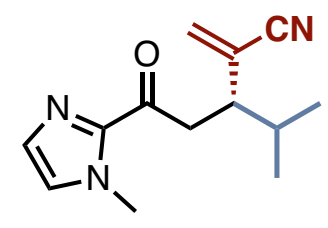

Molecular formula: $\mathrm{C}_{13} \mathrm{H}_{17} \mathrm{~N}_{3} \mathrm{O}$

$\mathbf{M W}=231.30 \mathrm{~g} \cdot \mathrm{mol}^{-1}$

The title compound was isolated as an amorphous solid (34 mg, $0.146 \mathrm{mmol}, 54 \%, 84 \%$ ee) using general procedure F. Purified by flash column chromatography over silica gel $\left(\mathrm{Hex} / \mathrm{Et}_{2} \mathrm{O}=1: 1\right)$.

${ }^{1}$ H NMR $\left(400 \mathrm{MHz}, \mathrm{CDCl}_{3}\right) \delta 7.14(\mathrm{~d}, J=0.9 \mathrm{~Hz}, 1 \mathrm{H}), 7.03(\mathrm{~d}, \mathrm{~J}=0.9 \mathrm{~Hz}, 1 \mathrm{H}), 5.89(\mathrm{~s}, 1 \mathrm{H}), 5.76$ (s, $1 \mathrm{H}), 3.97(\mathrm{~s}, 3 \mathrm{H}), 3.47$ (dd, $J=17.3,10.0 \mathrm{~Hz}, 1 \mathrm{H}), 3.32$ (dd, $J=17.3,4.1 \mathrm{~Hz}, 1 \mathrm{H}), 2.80(\mathrm{~m}$, $1 \mathrm{H}), 1.89(\mathrm{~m}, 1 \mathrm{H}), 1.00(\mathrm{~d}, J=6.8 \mathrm{~Hz}, 3 \mathrm{H}), 0.99(\mathrm{~d}, J=6.8 \mathrm{~Hz}, 3 \mathrm{H})$.

${ }^{13} \mathrm{C}$ NMR $\left(101 \mathrm{MHz}, \mathrm{CDCl}_{3}\right) \delta 190.6,143.0,131.7,129.4,127.3,125.6,118.3,46.4,39.8,36.3$, $31.1,20.3,19.9$.

HRMS (ESI) m/z: [M+H] $]^{+}$calcd for $\mathrm{C}_{13} \mathrm{H}_{18} \mathrm{~N}_{3} \mathrm{O}$ 232.1450; found: 232.1449 .

IR $\left(\mathrm{cm}^{-1}\right):$ 3004, 2929, 2224, 1674, 1611, 1467, 1411, 1292, 1158.

$[\boldsymbol{a}]_{D}^{20}=-75.8\left(c 0.37, \mathrm{CH}_{2} \mathrm{Cl}_{2}\right)$

HPLC analysis: carried out using a mixture of hexane and isopropyl alcohol (85:15 isocratic) at $25^{\circ} \mathrm{C}$ on an IC column, with UV detection $(\lambda=280 \mathrm{~nm})$. Retention times: 10.5 and 11.9 $\min$. 
${ }^{1} \mathrm{H}-\mathrm{NMR}$ of compound $3 \mathrm{n}\left(400 \mathrm{MHz}, \mathrm{CDCl}_{3}\right)$
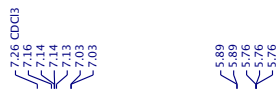

م.

S

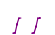<smiles>C=C(C#N)[C@H](CC(=O)c1nccn1C)C(C)C</smiles>

$3 n$

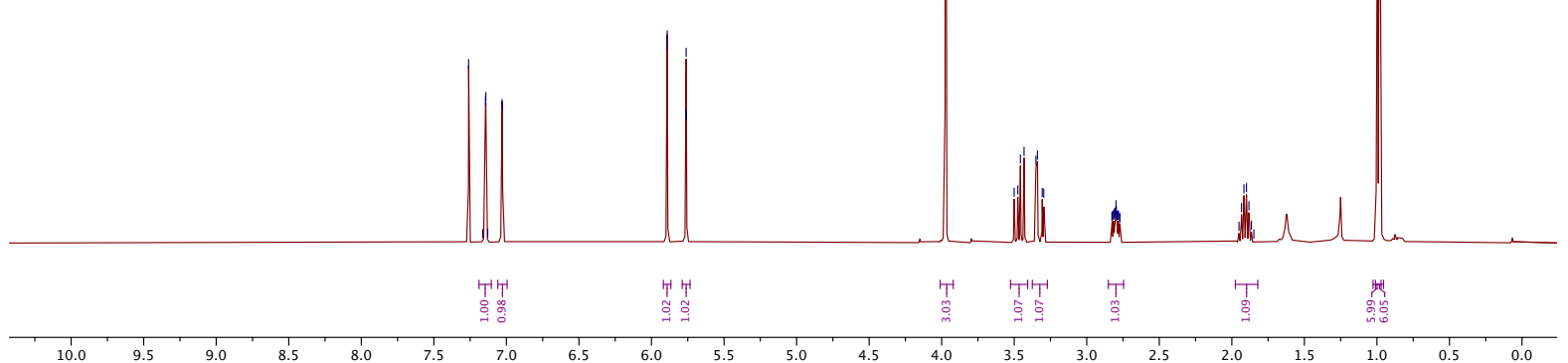

${ }^{13} \mathrm{C}$-NMR of compound $3 \mathrm{n}\left(101 \mathrm{MHz}, \mathrm{CDCl}_{3}\right)$

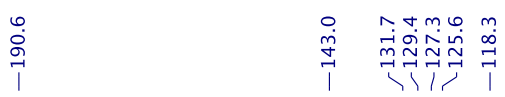

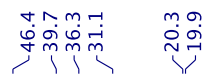



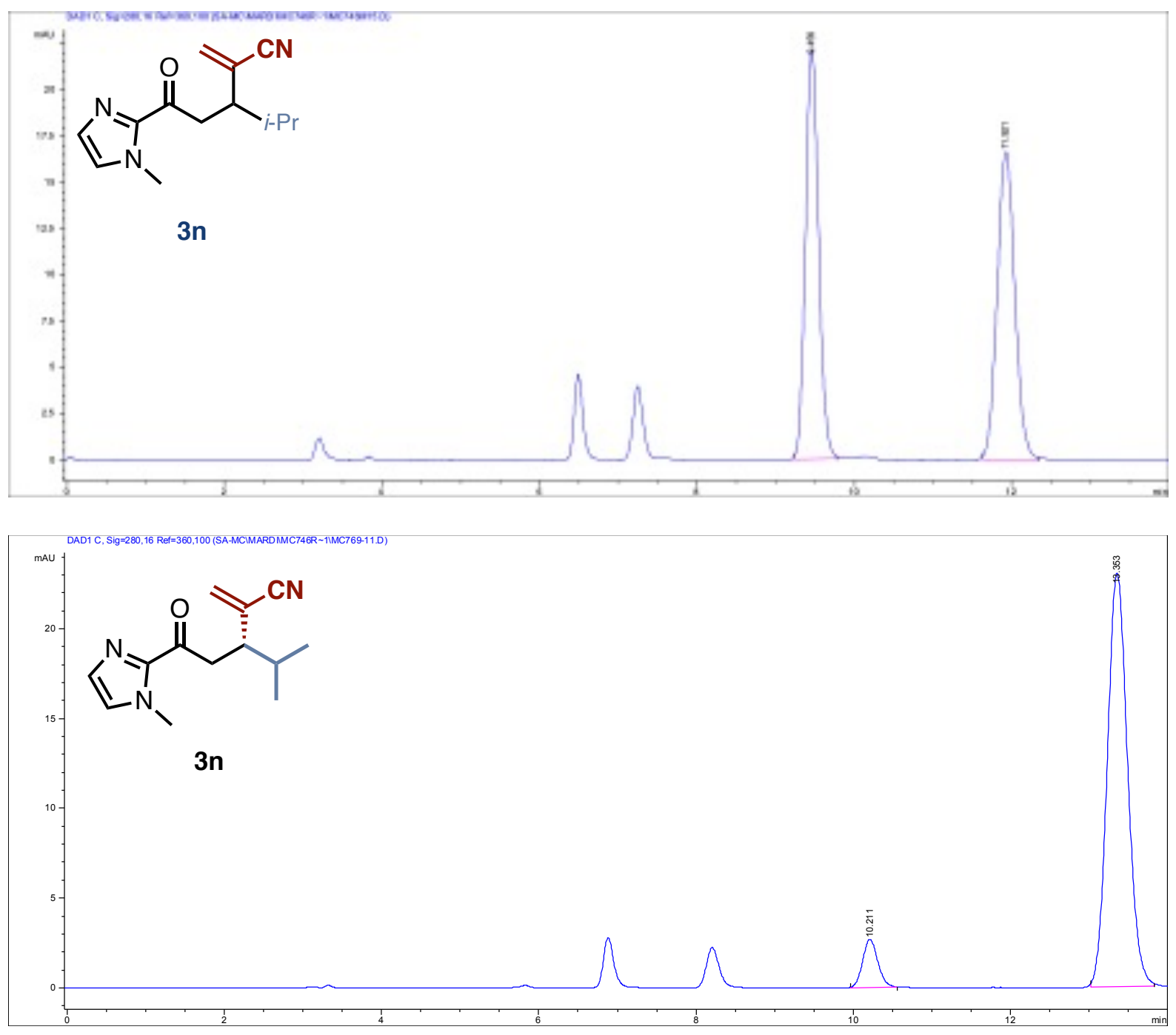

\begin{tabular}{|c|c|c|c|c|c|c|}
\hline $\begin{array}{c}\text { Peak } \\
\quad \#\end{array}$ & $\begin{array}{c}\text { RetTime } \\
\text { [min] }\end{array}$ & Type & $\begin{array}{l}\text { Width } \\
\text { [min] }\end{array}$ & $\begin{array}{c}\text { Area } \\
{\left[\mathrm{mAU}^{*} \mathrm{~S}\right]}\end{array}$ & $\begin{array}{l}\text { Height } \\
{[\mathrm{mAU}]}\end{array}$ & $\begin{array}{c}\text { Area } \\
\%\end{array}$ \\
\hline-- & ------ & & ----- & - - - - - - - - & ------ & ---1 \\
\hline 1 & 10.211 & BB & 0.2088 & 35.96603 & 2.67571 & 8.0376 \\
\hline 2 & 13.353 & BB & 0.2763 & 411.50620 & 23.04949 & 91.9624 \\
\hline
\end{tabular}


3-Cyclohexyl-5-(1-methyl-1H-imidazol-2-yl)-2-methylene-5-oxopentanenitrile (30)

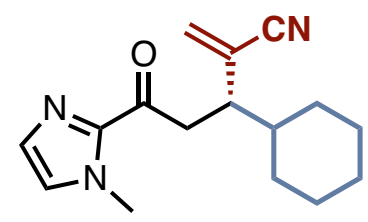

Molecular formula: $\mathrm{C}_{16} \mathrm{H}_{21} \mathrm{~N}_{3} \mathrm{O}$

$\mathrm{MW}=271.36 \mathrm{~g} \cdot \mathrm{mol}^{-1}$

The title compound was isolated as an amorphous solid (45 mg, $0.165 \mathrm{mmol}, 61 \%, 76 \%$ ee) using general procedure F. Purified by flash column chromatography over silica gel $\left(\mathrm{Hex} / \mathrm{Et}_{2} \mathrm{O}=3: 7\right)$.

${ }^{1}$ H NMR $\left(400 \mathrm{MHz}, \mathrm{CDCl}_{3}\right) \delta 7.14(\mathrm{~d}, J=1.0 \mathrm{~Hz}, 1 \mathrm{H}), 7.02(\mathrm{~d}, J=1.0 \mathrm{~Hz}, 1 \mathrm{H}), 5.88(\mathrm{~s}, 1 \mathrm{H}), 5.74$ (s, 1H), $3.97(\mathrm{~s}, 3 \mathrm{H}), 3.41(\mathrm{~d}, J=8.6 \mathrm{~Hz}, 1 \mathrm{H}), 3.39(\mathrm{~d}, J=5.6 \mathrm{~Hz}, 1 \mathrm{H}$ ), 2.82 (ddd, $J=8.6,7.5$, $5.6 \mathrm{~Hz}, 1 \mathrm{H}), 1.82-1.72(\mathrm{~m}, 4 \mathrm{H}), 1.67(\mathrm{~m}, 1 \mathrm{H}), 1.57-1.49(\mathrm{~m}, 2 \mathrm{H}), 1.22-1.10(\mathrm{~m}, 2 \mathrm{H}), 1.06-0.96$ $(\mathrm{m}, 2 \mathrm{H})$.

${ }^{13} \mathrm{C}$ NMR $\left(101 \mathrm{MHz}, \mathrm{CDCl}_{3}\right) \delta 190.7,143.0,131.6,129.4,127.3,125.7,118.4,45.7,40.6,39.8$, $36.3,30.8,30.4,26.4(2 C), 26.4$.

HRMS (ESI) m/z: [M+H] $]^{+}$calcd for $\mathrm{C}_{16} \mathrm{H}_{22} \mathrm{~N}_{3} \mathrm{O}$ 272.1663; found: 272.1663 .

IR $\left(\mathrm{cm}^{-1}\right)$ : 2929, 2855, 2227, 1678, 1466, 1452, 1411, 1292, 1158.

$[a]_{D}^{20}=-54.6\left(c 0.63, \mathrm{CH}_{2} \mathrm{Cl}_{2}\right)$

HPLC analysis: carried out using a mixture of hexane and isopropyl alcohol (80:20 isocratic) at $25{ }^{\circ} \mathrm{C}$ on an IC column, with UV detection $(\lambda=280 \mathrm{~nm})$. Retention times: 10.9 and 18.0 $\min$. 
${ }^{1} \mathrm{H}-\mathrm{NMR}$ of compound $30\left(400 \mathrm{MHz}, \mathrm{CDCl}_{3}\right)$

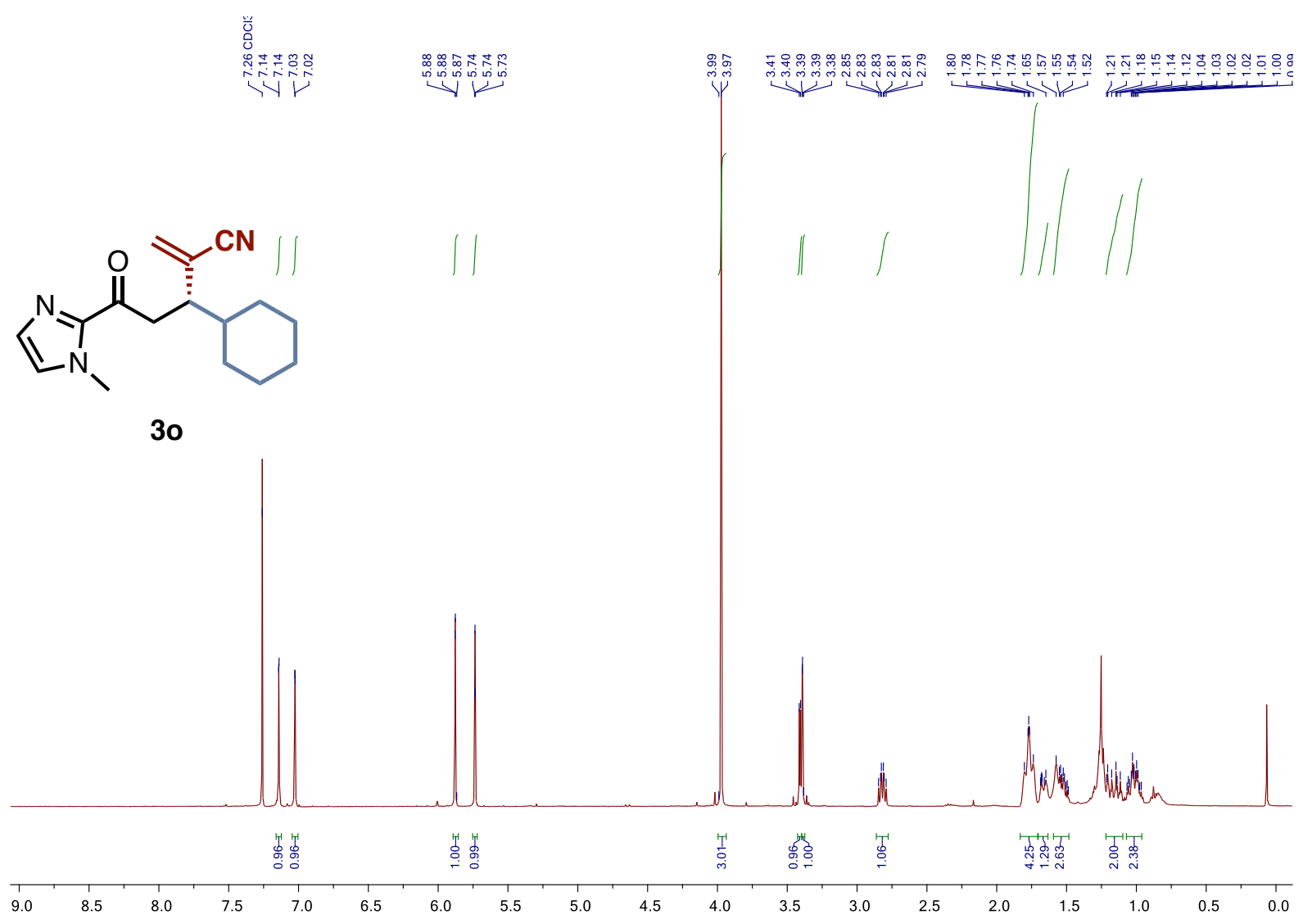

${ }^{13} \mathrm{C}-\mathrm{NMR}$ of compound $30\left(101 \mathrm{MHz}, \mathrm{CDCl}_{3}\right)$

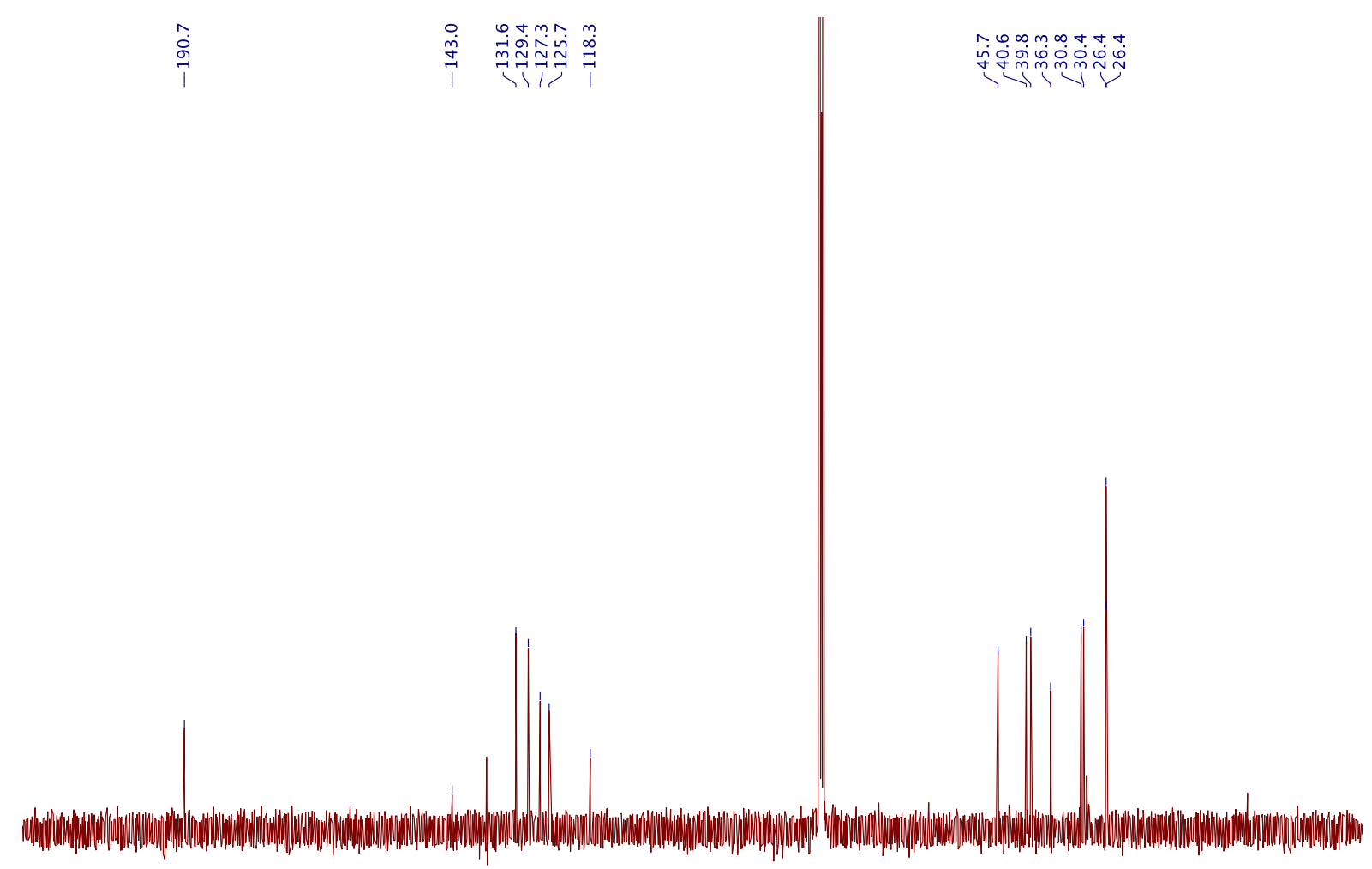

$\begin{array}{lllllllllllllllllllllll}210 & 200 & 190 & 180 & 170 & 160 & 150 & 140 & 130 & 120 & 110 & 100 & 90 & 80 & 70 & 60 & 50 & 40 & 30 & 20 & 10 & 0 & -10\end{array}$ 

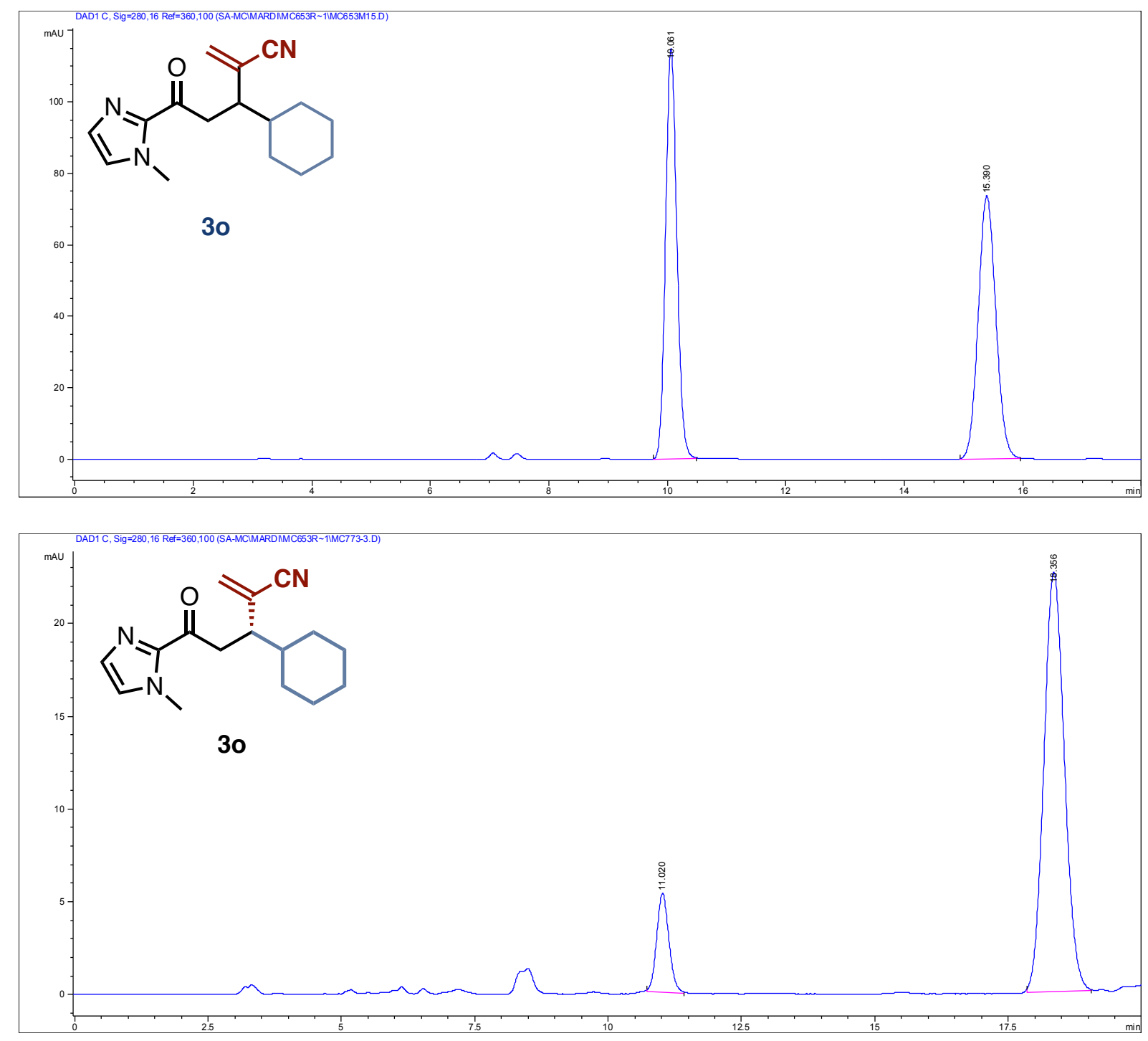

\begin{tabular}{|c|c|c|c|c|c|c|}
\hline $\begin{array}{c}\text { Peak } \\
\#\end{array}$ & $\begin{array}{c}\text { RetTime } \\
\text { [min] }\end{array}$ & Type & $\begin{array}{l}\text { Width } \\
\text { [min] }\end{array}$ & $\begin{array}{c}\text { Area } \\
{[\mathrm{mAU} * \mathrm{~s}]}\end{array}$ & $\begin{array}{l}\text { Height } \\
\text { [mAU] }\end{array}$ & $\begin{array}{c}\text { Area } \\
\%\end{array}$ \\
\hline & & & & & & \\
\hline 1 & & & & 84 & 848 & 12. \\
\hline 2 & 18.356 & $\mathrm{BB}$ & .4145 & 605.96112 & 22.62153 & 87.7624 \\
\hline
\end{tabular}


tert-Butyl 4-(2-cyano-5-(1-methyl-1H-imidazol-2-yl)-5-oxopent-1-en-3-yl)piperidine-1carboxylate (3p)

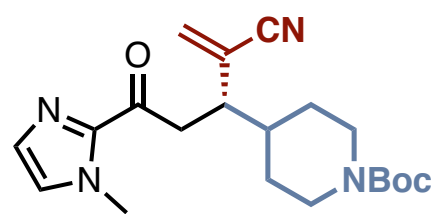

Molecular formula: $\mathrm{C}_{20} \mathrm{H}_{28} \mathrm{~N}_{4} \mathrm{O}_{3}$

$\mathrm{MW}=372.47 \mathrm{~g} \cdot \mathrm{mol}^{-1}$

The title compound was isolated as a beige solid (76 mg, $0.205 \mathrm{mmol}, 76 \%, \mathbf{7 2 \%}$ ee) using general procedure F. Purified by flash column chromatography over silica gel $($ Hex/EtOAc/Acetone $=5: 4: 1)$.

${ }^{1} \mathrm{H}$ NMR $\left(400 \mathrm{MHz}_{\mathrm{CDCl}}\right) \delta 7.14(\mathrm{~d}, J=0.9 \mathrm{~Hz}, 1 \mathrm{H}), 7.04(\mathrm{~d}, 0.9 \mathrm{~Hz}, 1 \mathrm{H}), 5.92(\mathrm{~s}, 1 \mathrm{H}), 5.78$ (s, 1H), 4.15 (br s, 2H), $3.98(\mathrm{~s}, 3 \mathrm{H}), 3.41(\mathrm{~d}, J=7.2 \mathrm{~Hz}, 2 \mathrm{H}), 2.85(\mathrm{q}, J=7.2 \mathrm{~Hz}, 1 \mathrm{H}), 2.68$ $(\mathrm{t}, J=12.7 \mathrm{~Hz}, 2 \mathrm{H}), 1.76-1.63(\mathrm{~m}, 4 \mathrm{H}), 1.58(\mathrm{~m}, 1 \mathrm{H}), 1.45(\mathrm{~s}, 9 \mathrm{H})$.

${ }^{13} \mathrm{C}$ NMR $\left(101 \mathrm{MHz}^{\mathrm{C}} \mathrm{CDCl}_{3}\right) \delta 189.9,154.6,142.7,132.2,129.4,127.3,124.8,117.8,79.5$, $45.1,39.5,38.8,36.1,29.9,29.5,28.5$.

HRMS (ESI) m/z: [M+H] $]^{+}$calcd for $\mathrm{C}_{20} \mathrm{H}_{29} \mathrm{~N}_{4} \mathrm{O}_{3} 373.2239$; found: 373.2250 .

IR $\left(\mathrm{cm}^{-1}\right):$ 2929, 2857, 2343, 2224, 1682, 1411, 1369, 1281, 1251, 1168.

$[a]_{D}^{20}=-18.8\left(c 2.53, \mathrm{CH}_{2} \mathrm{Cl}_{2}\right)$

HPLC analysis: carried out using a mixture of hexane and isopropyl alcohol (78:22 isocratic) at $1 \mathrm{ml} \cdot \mathrm{min}^{-1}$, at $30{ }^{\circ} \mathrm{C}$ on an IC column, with UV detection $(\lambda=280 \mathrm{~nm})$. Retention times: 44.64 and $49.02 \mathrm{~min}$. 
${ }^{1} \mathrm{H}-\mathrm{NMR}$ of compound $3 p\left(400 \mathrm{MHz}, \mathrm{CDCl}_{3}\right)$

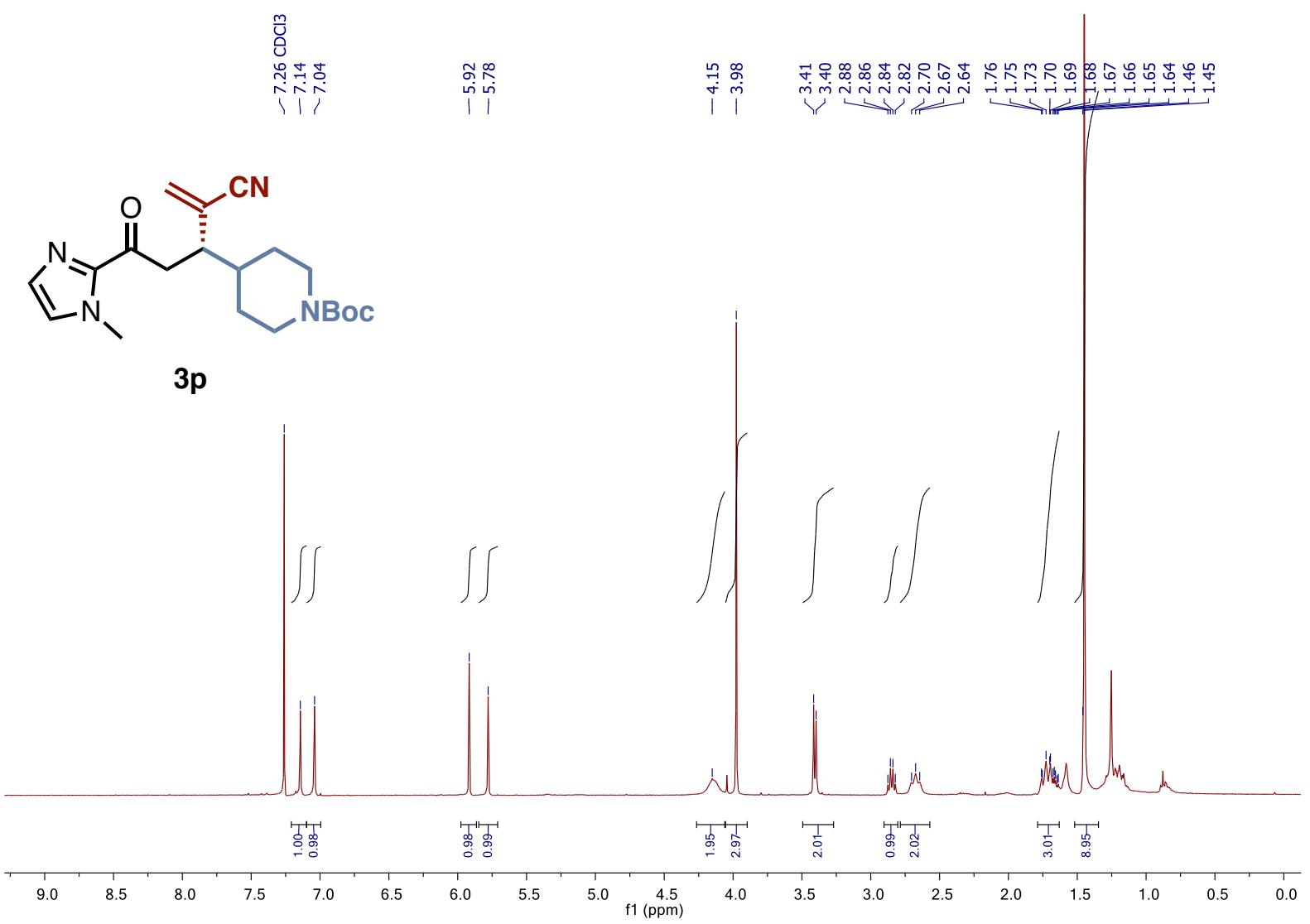

\section{${ }^{13} \mathrm{C}-\mathrm{NMR}$ of compound $3 \mathrm{p}\left(101 \mathrm{MHz}, \mathrm{CDCl}_{3}\right)$}

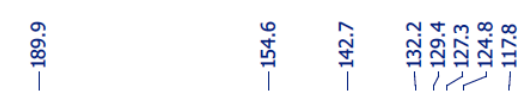

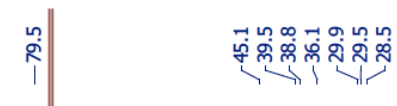

$\begin{array}{llllllllllllllllllllllll}210 & 200 & 190 & 180 & 170 & 160 & 150 & 140 & 130 & 120 & 110 & \begin{array}{c}100 \\ \mathrm{f} 1(\mathrm{ppm})\end{array} & 90 & 80 & 70 & 60 & 50 & 40 & 30 & 20 & 10 & 0 & -10\end{array}$ 

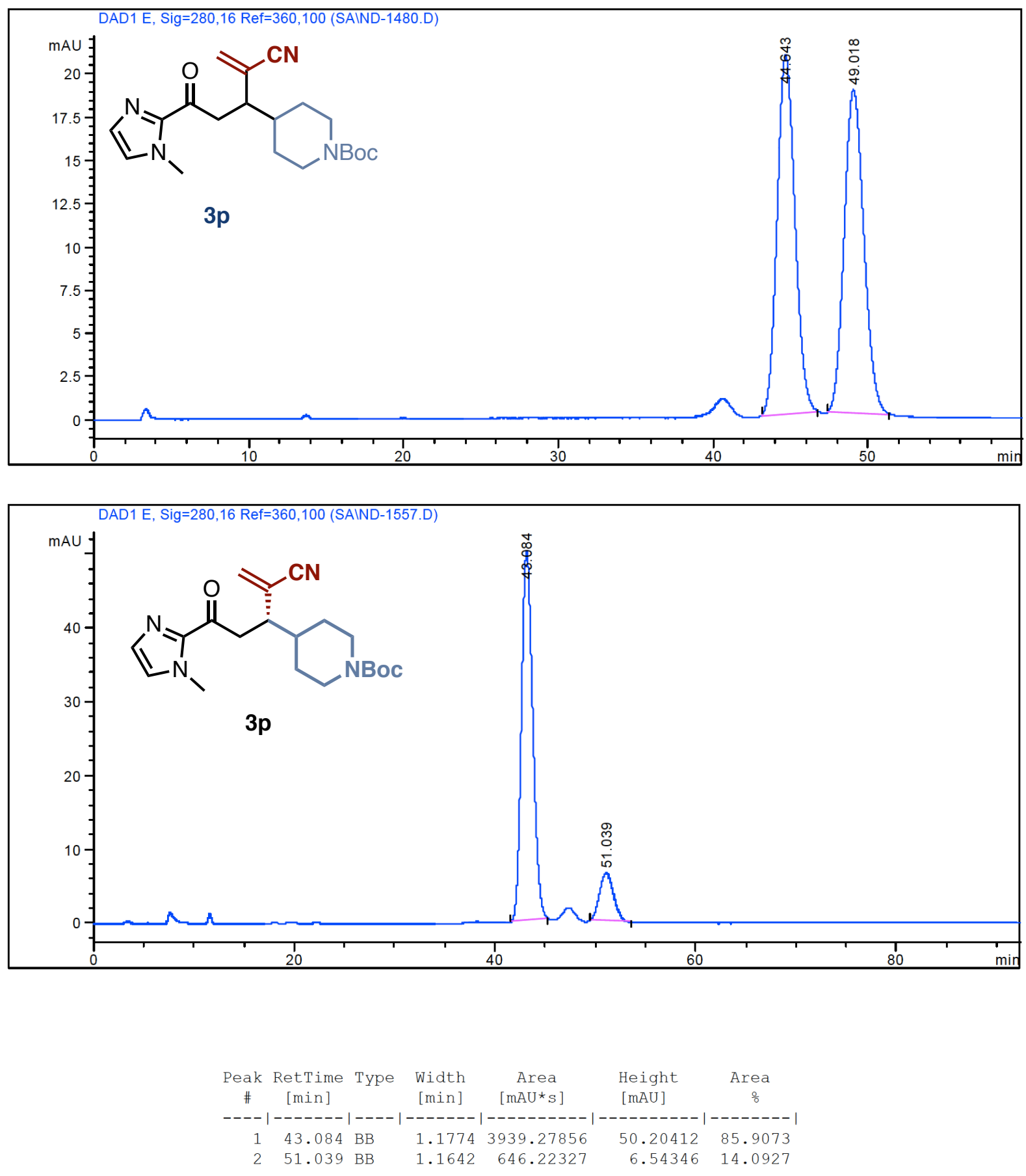
yl)pentanenitrile (3q)

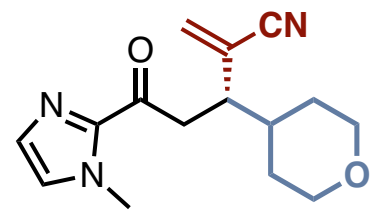

Molecular formula: $\mathrm{C}_{15} \mathrm{H}_{19} \mathrm{~N}_{3} \mathrm{O}_{2}$

$\mathrm{MW}=273.34 \mathrm{~g} \cdot \mathrm{mol}^{-1}$

The title compound was isolated as a clear oil (61 mg, $0.224 \mathrm{mmol}, 83 \%, 68 \%$ ee $)$ using general procedure F. Purified by flash column chromatography over silica gel $($ Hex/EtOAc/Acetone $=7: 2: 1)$.

${ }^{1} \mathrm{H}$ NMR $\left(400 \mathrm{MHz}, \mathrm{CDCl}_{3}\right) \delta 7.14(\mathrm{~d}, J=1.0 \mathrm{~Hz}, 1 \mathrm{H}), 7.03(\mathrm{~d}, 1.0 \mathrm{~Hz}, 1 \mathrm{H}), 5.91(\mathrm{~d}, J=0.6 \mathrm{~Hz}$, $1 \mathrm{H}), 5.79(\mathrm{~d}, J=0.7 \mathrm{~Hz}, 1 \mathrm{H}), 4.02-3.96(\mathrm{~m}, 2 \mathrm{H}), 3.97(\mathrm{~s}, 3 \mathrm{H}), 3.41-3.34(\mathrm{~m}, 4 \mathrm{H}), 2.82$ $(\mathrm{dt}, J=8.5,7.0 \mathrm{~Hz}, 1 \mathrm{H}), 1.79(\mathrm{~m}, 1 \mathrm{H}), 1.73-1.61(\mathrm{~m}, 3 \mathrm{H}), 1.43-1.29(\mathrm{~m}, 2 \mathrm{H})$.

${ }^{13} \mathrm{C}$ NMR $\left(101 \mathrm{MHz}, \mathrm{CDCl}_{3}\right) \delta 190.0,142.7,132.3,129.4,127.3,124.7,117.7,67.8,67.8$, $45.6,39.3,37.6,36.1,30.9,30.4$.

HRMS (ESI) m/z: [M+H] ${ }^{+}$calcd for $\mathrm{C}_{15} \mathrm{H}_{20} \mathrm{~N}_{3} \mathrm{O}_{2} 274.1555$; found: 274.1566 .

IR $\left(\mathrm{cm}^{-1}\right):$ 3130, 2939, 2848, 2224, 1676, 1469, 1410, 1095

$[a]_{D}^{20}=-9.8\left(c\right.$ 1.9, $\left.\mathrm{CH}_{2} \mathrm{Cl}_{2}\right)$

HPLC analysis: carried out using a mixture of hexane and isopropyl alcohol (70:30 isocratic) at $1 \mathrm{ml} \cdot \mathrm{min}^{-1}$, at $30{ }^{\circ} \mathrm{C}$ on an IC column, with UV detection $(\lambda=280 \mathrm{~nm})$. Retention times: 25.65 and $29.49 \mathrm{~min}$. 


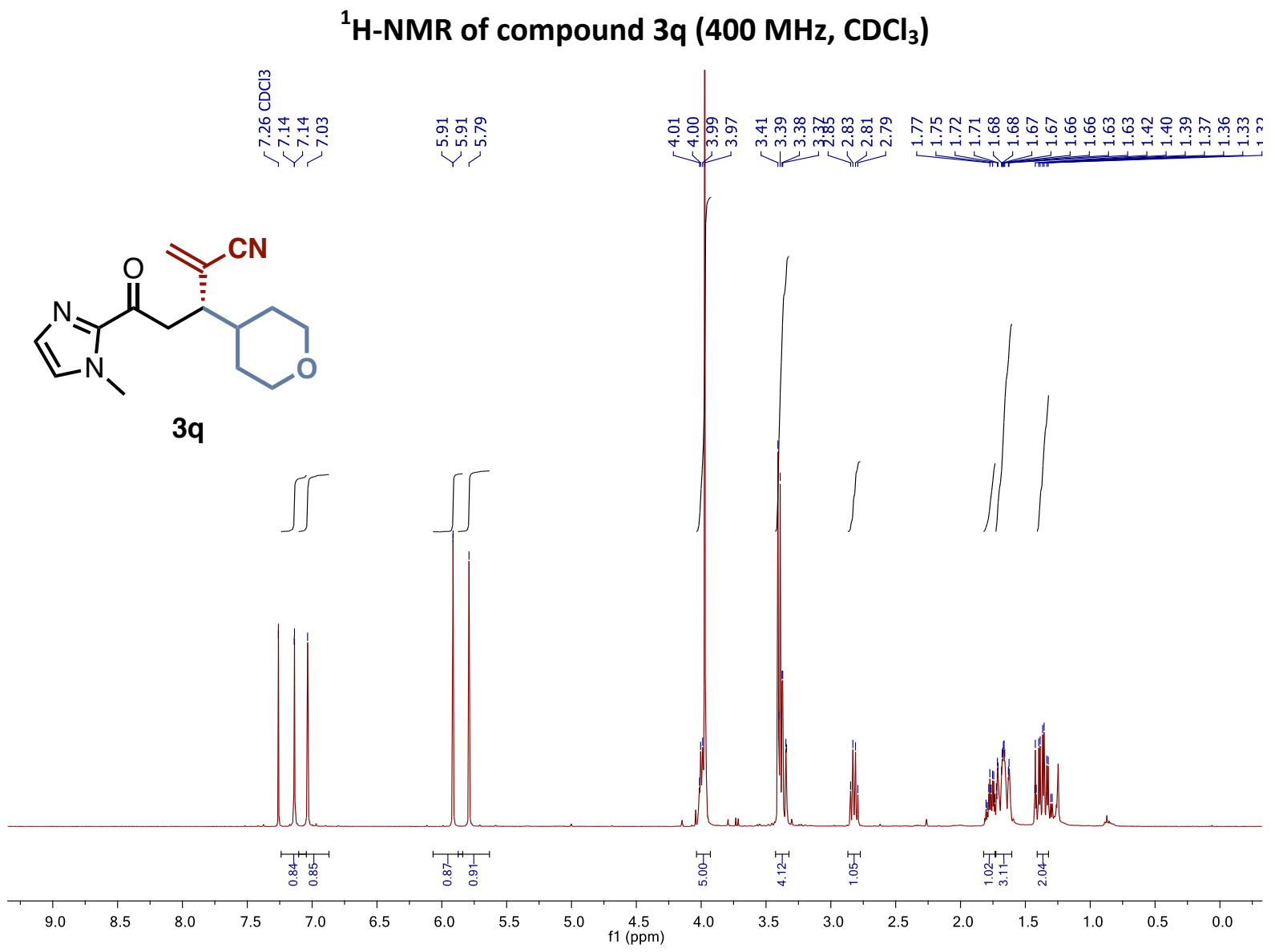

${ }^{13} \mathrm{C}-\mathrm{NMR}$ of compound $3 \mathrm{q}\left(101 \mathrm{MHz}, \mathrm{CDCl}_{3}\right)$

每

齐

$\begin{array}{lllllllllllllllllllllllllllllllll}210 & 200 & 190 & 180 & 170 & 160 & 150 & 140 & 130 & 120 & 110 & 100 & 90 & 80 & 70 & 60 & 50 & 40 & 30 & 20 & 10 & 0 & -10\end{array}$ 

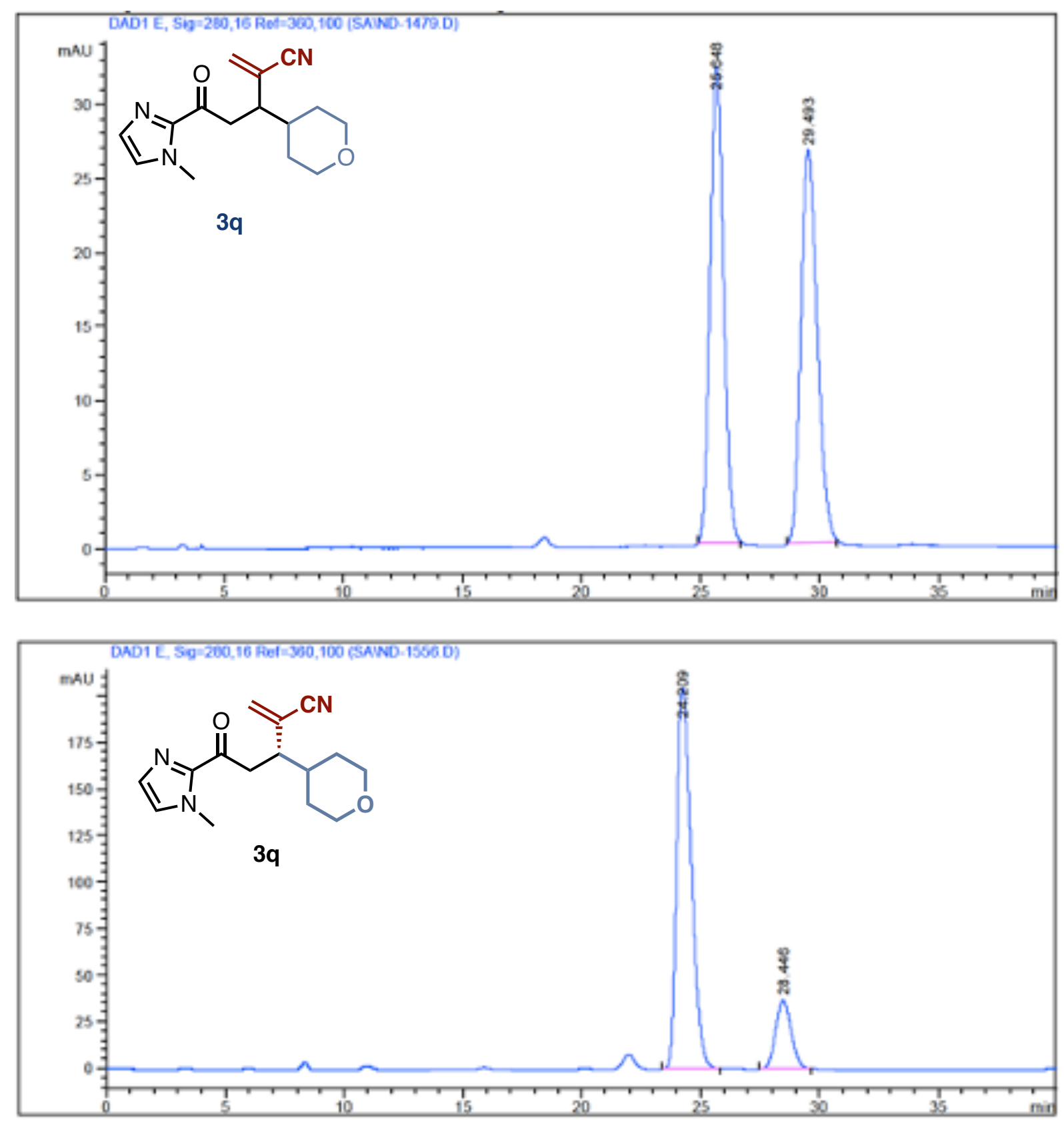

\begin{tabular}{|c|c|c|c|c|c|c|}
\hline $\begin{array}{l}\text { eak } \\
\#\end{array}$ & $\begin{array}{l}\text { RetTime } \\
{[\text { min] }}\end{array}$ & Type & $\begin{array}{l}\text { Width } \\
\text { [min] }\end{array}$ & $\begin{array}{c}\text { Area } \\
{[\mathrm{mAU} * \mathrm{~S}]}\end{array}$ & $\begin{array}{l}\text { Height } \\
{[\mathrm{mAU}]}\end{array}$ & $\begin{array}{c}\text { Area } \\
\quad \frac{\circ}{6}\end{array}$ \\
\hline 1 & & & & & & \\
\hline 2 & 28.446 & $\mathrm{BB}$ & 0.7103 & 1683.35046 & 36.75928 & 16.1950 \\
\hline
\end{tabular}




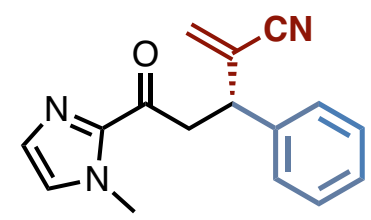

Molecular formula: $\mathrm{C}_{16} \mathrm{H}_{15} \mathrm{~N}_{3} \mathrm{O}$

$$
\mathrm{MW}=265.32 \mathrm{~g} \cdot \mathrm{mol}^{-1}
$$

The title compound was isolated as an off-white solid (58 mg, $0.219 \mathrm{mmol}, 81 \%, 80 \%$ ee ) using general procedure F. Purified by flash column chromatography over silica gel $($ Hex/EtOAc/Acetone $=7: 2: 1)$.

${ }^{1} \mathbf{H}$ NMR $\left(400 \mathrm{MHz}, \mathrm{CDCl}_{3}\right) \delta$ 7.36-7.30 (m, 4H), $7.26(\mathrm{~m}, 1 \mathrm{H}), 7.14(\mathrm{~d}, J=1.0 \mathrm{~Hz}, 1 \mathrm{H}), 7.02$ $(\mathrm{d}, J=1.0 \mathrm{~Hz}, 1 \mathrm{H}), 5.91(\mathrm{~s}, 1 \mathrm{H}), 5.85(\mathrm{~s}, 1 \mathrm{H}), 4.32(\mathrm{t}, J=7.5 \mathrm{~Hz}, 1 \mathrm{H}), 3.94(\mathrm{~s}, 3 \mathrm{H}), 3.82$ (dd, $J=17.3,7.5 \mathrm{~Hz}, 1 \mathrm{H}$ ), 3.75 (dd, $J=17.3,7.5 \mathrm{~Hz}, 1 \mathrm{H}$ ).

${ }^{13} \mathrm{C}$ NMR $\left(101 \mathrm{MHz}, \mathrm{CDCl}_{3}\right) \delta 189.1,142.8,139.4,130.3,129.5,129.1$ (2C), $128.0(2 \mathrm{C}), 127.8$, $127.5,126.6,118.1,45.0,42.3,36.2$.

HRMS (ESI) m/z: [M+H] $]^{+}$calcd for $\mathrm{C}_{16} \mathrm{H}_{16} \mathrm{~N}_{3} \mathrm{O}$ 266.1293; found: 266.1276 .

IR $\left(\mathrm{cm}^{-1}\right): 2957,2926,2857,2224,1678,1411,1292,1158,994,918$.

$[a]_{D}^{20}=-47.4\left(c 0.63, \mathrm{CH}_{2} \mathrm{Cl}_{2}\right)$

HPLC analysis: carried out using a mixture of hexane and isopropyl alcohol (70:30 isocratic), at $1 \mathrm{~mL} \cdot \mathrm{min}^{-1}$, at $25{ }^{\circ} \mathrm{C}$ on an IC column, with UV detection ( $\lambda=280 \mathrm{~nm}$ ). Retention times: 9.3 and $11.4 \mathrm{~min}$. 
${ }^{1} \mathrm{H}-\mathrm{NMR}$ of compound $3 \mathrm{r}\left(400 \mathrm{MHz}, \mathrm{CDCl}_{3}\right)$

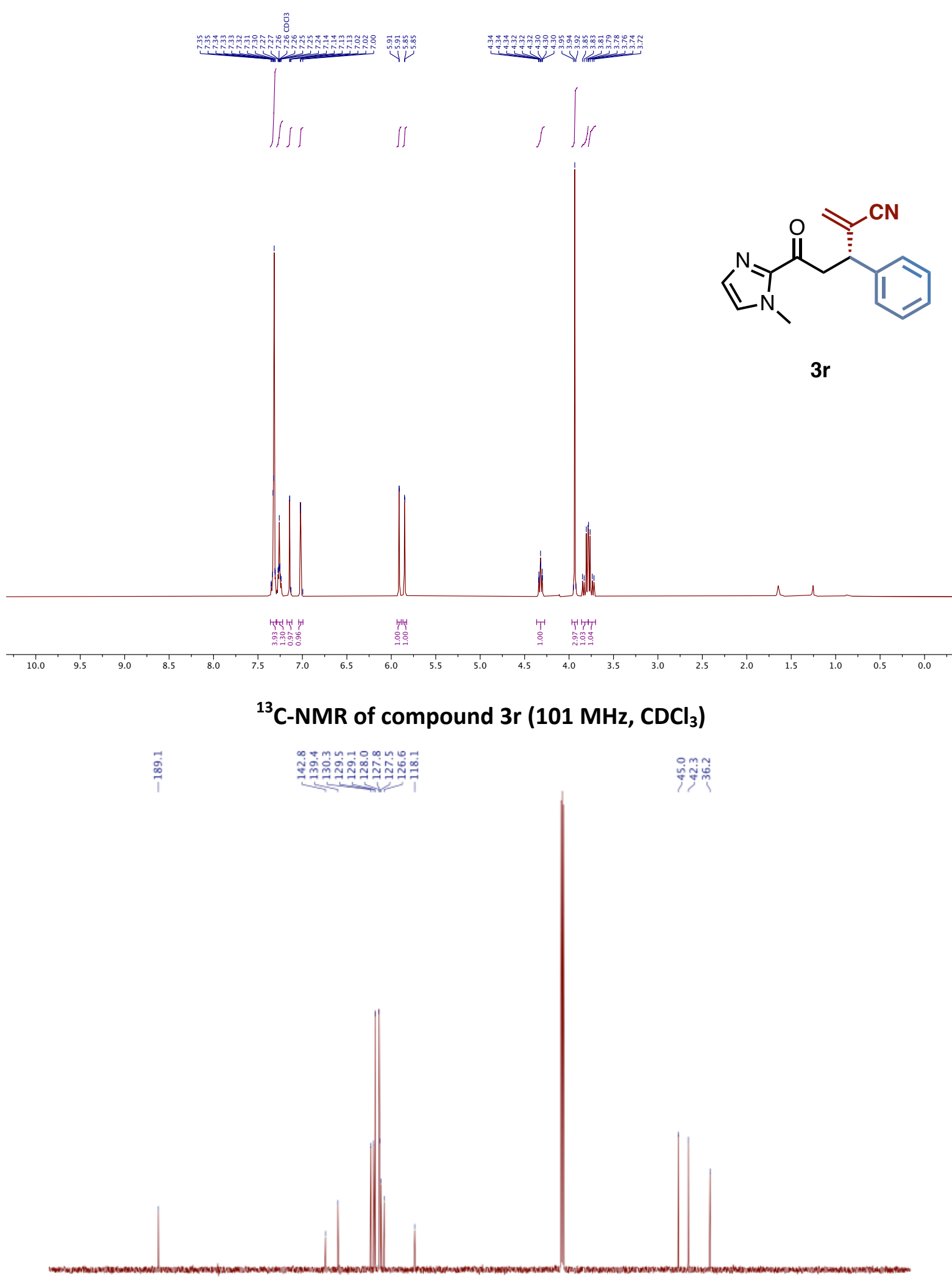

$\begin{array}{lllllllllllllllllllllll}210 & 200 & 190 & 180 & 170 & 160 & 150 & 140 & 130 & 120 & 110 & 100 & 90 & 80 & 70 & 60 & 50 & 40 & 30 & 20 & 10 & 0 & -10\end{array}$ 

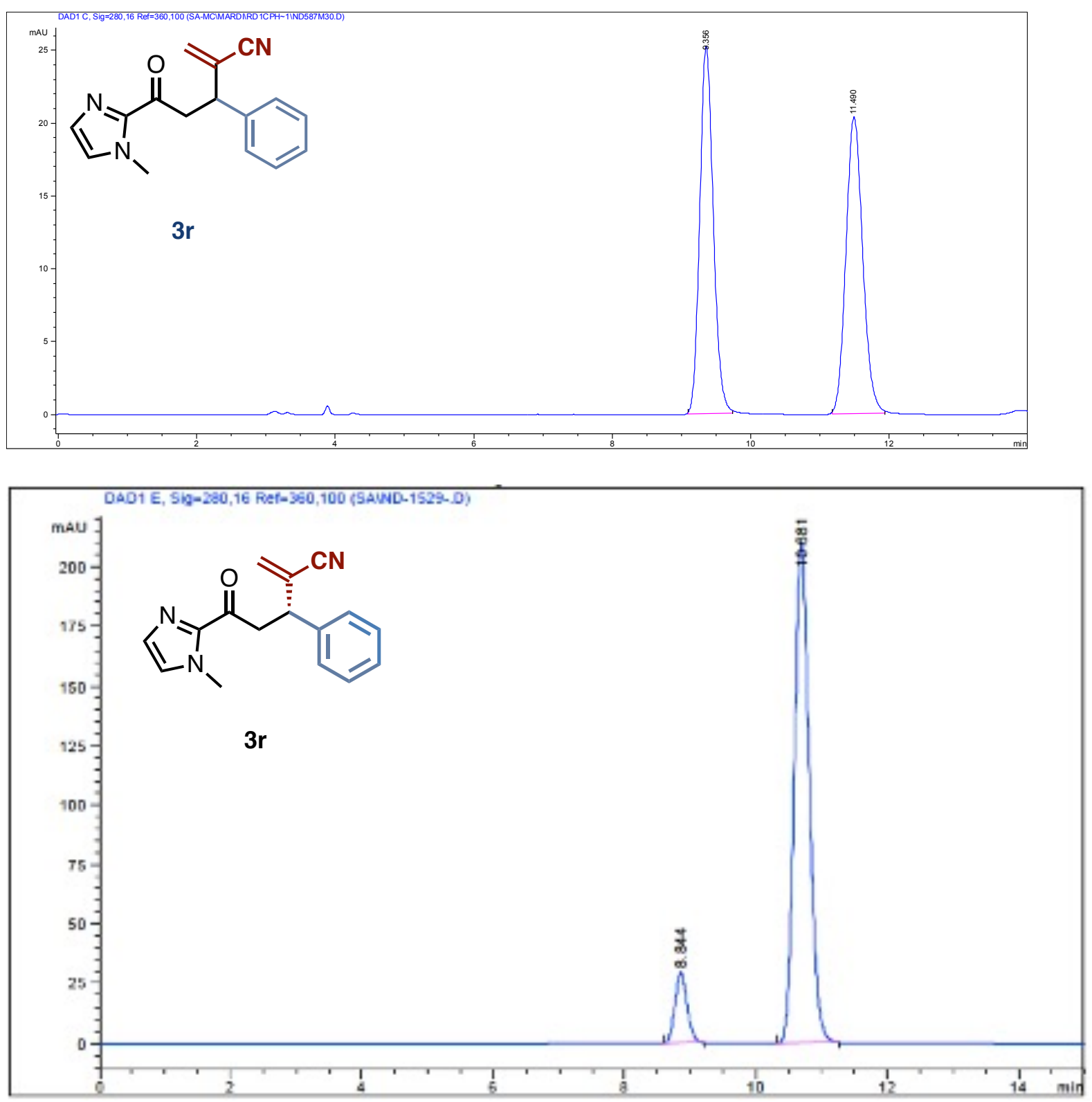

\begin{tabular}{|c|c|c|c|c|c|c|}
\hline $\begin{array}{c}\text { Peak } \\
\quad \neq\end{array}$ & $\begin{array}{c}\text { RetTime } \\
\text { [min] }\end{array}$ & Type & $\begin{array}{l}\text { Width } \\
\text { [min] }\end{array}$ & $\begin{array}{c}\text { Area } \\
{\left[\mathrm{mAU}^{*} \mathrm{~s}\right]}\end{array}$ & $\begin{array}{l}\text { Height } \\
\text { [mAU] }\end{array}$ & $\begin{array}{c}\text { Area } \\
\&\end{array}$ \\
\hline 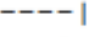 & & & & ----- & ---- & 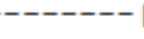 \\
\hline 1 & 8.844 & BB & 0.1983 & 384.55099 & 29.85496 & 10.3647 \\
\hline 2 & 10.681 & BB & 0.2443 & 3325.65186 & 210.52946 & 89.6353 \\
\hline
\end{tabular}




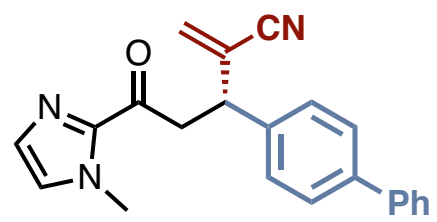

Molecular formula: $\mathrm{C}_{22} \mathrm{H}_{19} \mathrm{~N}_{3} \mathrm{O}$

$\mathrm{MW}=341.41 \mathrm{~g} \cdot \mathrm{mol}^{-1}$

The title compound was isolated as an off-white wax (64 mg, $0.186 \mathrm{mmol}, 69 \%, \mathbf{8 1 \%}$ ee ) using general procedure F. Purified by flash column chromatography over silica gel (Toluene/Acetone $=94: 6$ ).

${ }^{1}$ H NMR $\left(400 \mathrm{MHz}, \mathrm{CDCl}_{3}\right) \delta$ 7.58-7.54 (m, 4H), $7.41(\mathrm{td}, J=8.4,6.7 \mathrm{~Hz}, 4 \mathrm{H}), 7.34(\mathrm{~m}, 1 \mathrm{H})$, $7.16(\mathrm{~d}, J=0.9 \mathrm{~Hz}, 1 \mathrm{H}), 7.03(\mathrm{~d}, J=0.8 \mathrm{~Hz}, 1 \mathrm{H}), 5.94(\mathrm{~d}, J=0.9 \mathrm{~Hz}, 1 \mathrm{H}), 5.90(\mathrm{~d}, J=1.3 \mathrm{~Hz}$, $1 \mathrm{H}), 4.37(\mathrm{t}, J=7.4 \mathrm{~Hz}, 1 \mathrm{H}), 3.95(\mathrm{~s}, 3 \mathrm{H}), 3.80(\mathrm{dd}, J=7.4,0.9 \mathrm{~Hz}, 2 \mathrm{H})$.

${ }^{13} \mathrm{C}$ NMR $\left(101 \mathrm{MHz}, \mathrm{CDCl}_{3}\right) \delta 189.0,142.7,140.5,138.2,128.8,128.3,127.6,127.0,118.0$, $44.5,42.1,36.1$.

HRMS (ESI) m/z: [M+H] $]^{+}$calcd for $\mathrm{C}_{22} \mathrm{H}_{20} \mathrm{~N}_{3} \mathrm{O}$ 342.1606; found: 342.1591 .

IR $\left(\mathrm{cm}^{-1}\right):$ 3032, 2957, 2926, 2855, 2224, 1676, 1490, 1410, 1283, 1112.

$[\boldsymbol{a}]_{\boldsymbol{D}}^{20}=-9.6\left(c 2.37, \mathrm{CH}_{2} \mathrm{Cl}_{2}\right)$

HPLC analysis: carried out using a mixture of hexane and isopropyl alcohol (80:20 isocratic), at $1 \mathrm{~mL} \cdot \mathrm{min}^{-1}$, at $30^{\circ} \mathrm{C}$ on an IC column, with UV detection $(\lambda=280 \mathrm{~nm})$. Retention times: 14.63 and $17.79 \mathrm{~min}$. 
${ }^{1} \mathrm{H}-\mathrm{NMR}$ of compound 3s (400 $\mathrm{MHz}, \mathrm{CDCl}_{3}$ )

苍

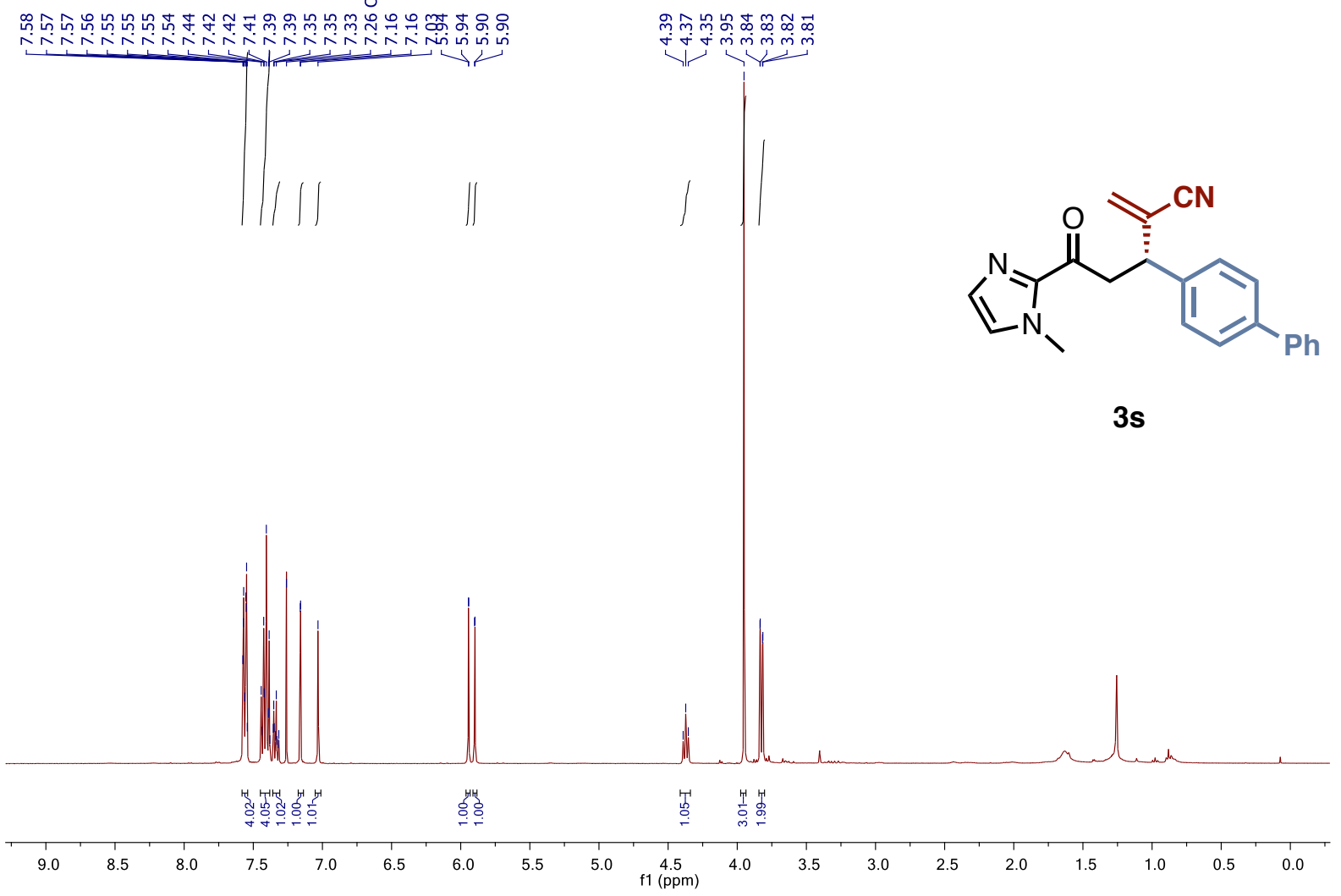

${ }^{13} \mathrm{C}-\mathrm{NMR}$ of compound 3s (101 MHz, $\mathrm{CDCl}_{3}$ )

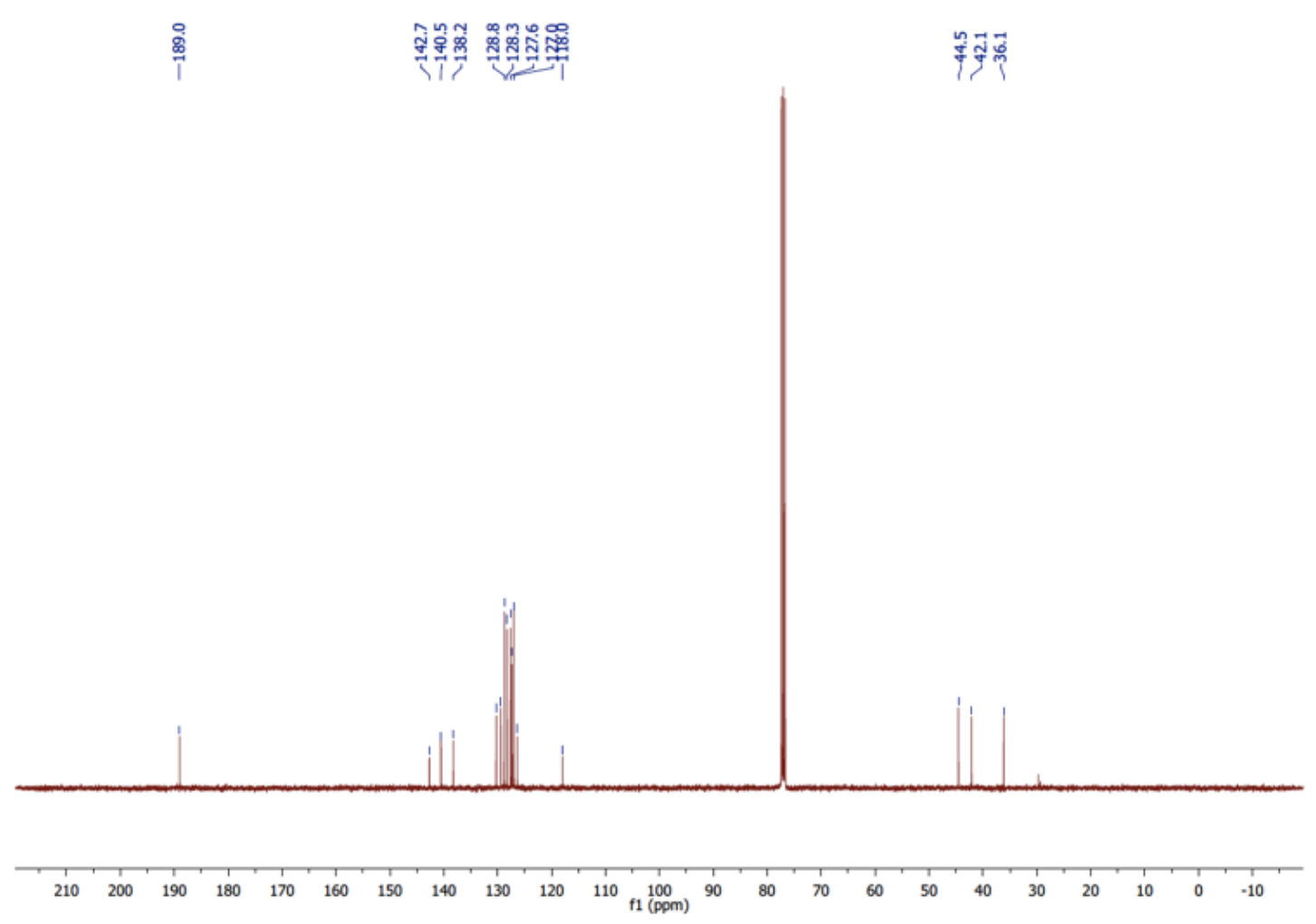



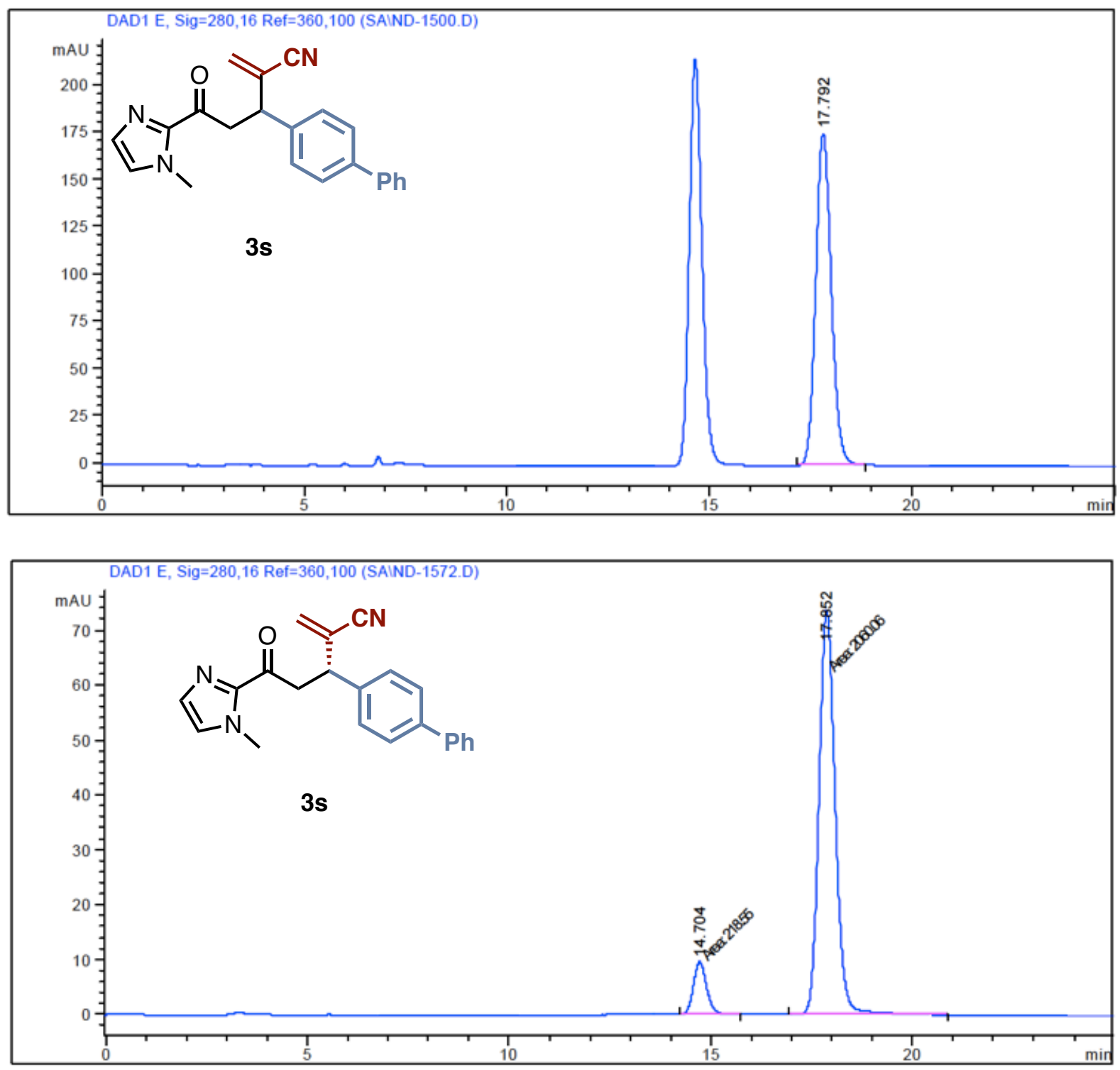

\begin{tabular}{|c|c|c|c|c|c|c|}
\hline $\begin{array}{c}\text { Peak } \\
\quad \neq\end{array}$ & $\begin{array}{c}\text { RetTime } \\
\text { [min] }\end{array}$ & Type & $\begin{array}{c}\text { Width } \\
\text { [min] }\end{array}$ & $\begin{array}{c}\text { Area } \\
{\left[\mathrm{mAU}{ }^{\star} \mathrm{s}\right]}\end{array}$ & $\begin{array}{l}\text { Height } \\
\text { [mAU] }\end{array}$ & $\begin{array}{c}\text { Area } \\
\text { \& }\end{array}$ \\
\hline & & & & & & \\
\hline 1 & & & & 218.55011 & 4660 & 14 \\
\hline 2 & 17.852 & MM & 0.4667 & 2060.06274 & 73.57252 & 90.4086 \\
\hline
\end{tabular}




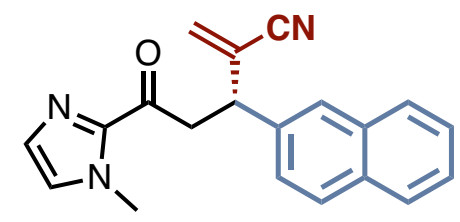

Molecular formula: $\mathrm{C}_{20} \mathrm{H}_{17} \mathrm{~N}_{3} \mathrm{O}$

$\mathbf{M W}=315.38 \mathrm{~g} \cdot \mathrm{mol}^{-1}$

The title compound was isolated as an off-white wax (50 mg, $0.159 \mathrm{mmol}, 59 \%, 85 \%$ ee) using general procedure F. Purified by flash column chromatography over silica gel $($ Hex/EtOAc/Acetone $=7: 2: 1)$.

${ }^{1} \mathbf{H}$ NMR $\left(400 \mathrm{MHz}, \mathrm{CDCl}_{3}\right) \delta$ 7.83-7.79 (m, 4H), 7.50-7.42 (m, 2H), $7.16(\mathrm{~d}, J=0.9 \mathrm{~Hz}, 1 \mathrm{H})$, $7.02(\mathrm{~d}, J=1.0 \mathrm{~Hz}, 1 \mathrm{H}), 5.96(\mathrm{~d}, J=0.9 \mathrm{~Hz}, 1 \mathrm{H}), 5.90(\mathrm{~d}, J=1.4 \mathrm{~Hz}, 1 \mathrm{H}), 4.50(\mathrm{t}, J=7.4 \mathrm{~Hz}, 1 \mathrm{H})$, $3.92(\mathrm{~s}, 3 \mathrm{H}), 3.91(\mathrm{~s}, 1 \mathrm{H}), 3.89(\mathrm{~d}, J=1.0 \mathrm{~Hz}, 1 \mathrm{H})$.

${ }^{13} \mathrm{C}$ NMR $\left(101 \mathrm{MHz}, \mathrm{CDCl}_{3}\right) \delta 188.9,142.7,136.6,133.5,132.8,130.4,129.4,128.8,128.0$, $127.6,127.4,126.8,126.4,126.3,126.1,125.9,118.0,44.9,42.1,36.1$, 29.7.

HRMS (ESI) m/z: [M+H] calcd for $\mathrm{C}_{20} \mathrm{H}_{18} \mathrm{~N}_{3} \mathrm{O}$ 316.1450; found: 316.1429 .

IR $\left(\mathrm{cm}^{-1}\right):$ 3056, 2957, 2928, 2341, 2225, 1687, 1410, 1268.

$[\boldsymbol{a}]_{\boldsymbol{D}}^{\mathbf{2 0}}=-17.4\left(\mathrm{c} 1.80, \mathrm{CH}_{2} \mathrm{Cl}_{2}\right)$

HPLC analysis: carried out using a mixture of hexane and isopropyl alcohol (80:20 isocratic), at $1 \mathrm{~mL} \cdot \mathrm{min}^{-1}$, at $30{ }^{\circ} \mathrm{C}$ on an IC column, with UV detection $(\lambda=280 \mathrm{~nm})$. Retention times: 14.11 and $17.59 \mathrm{~min}$. 


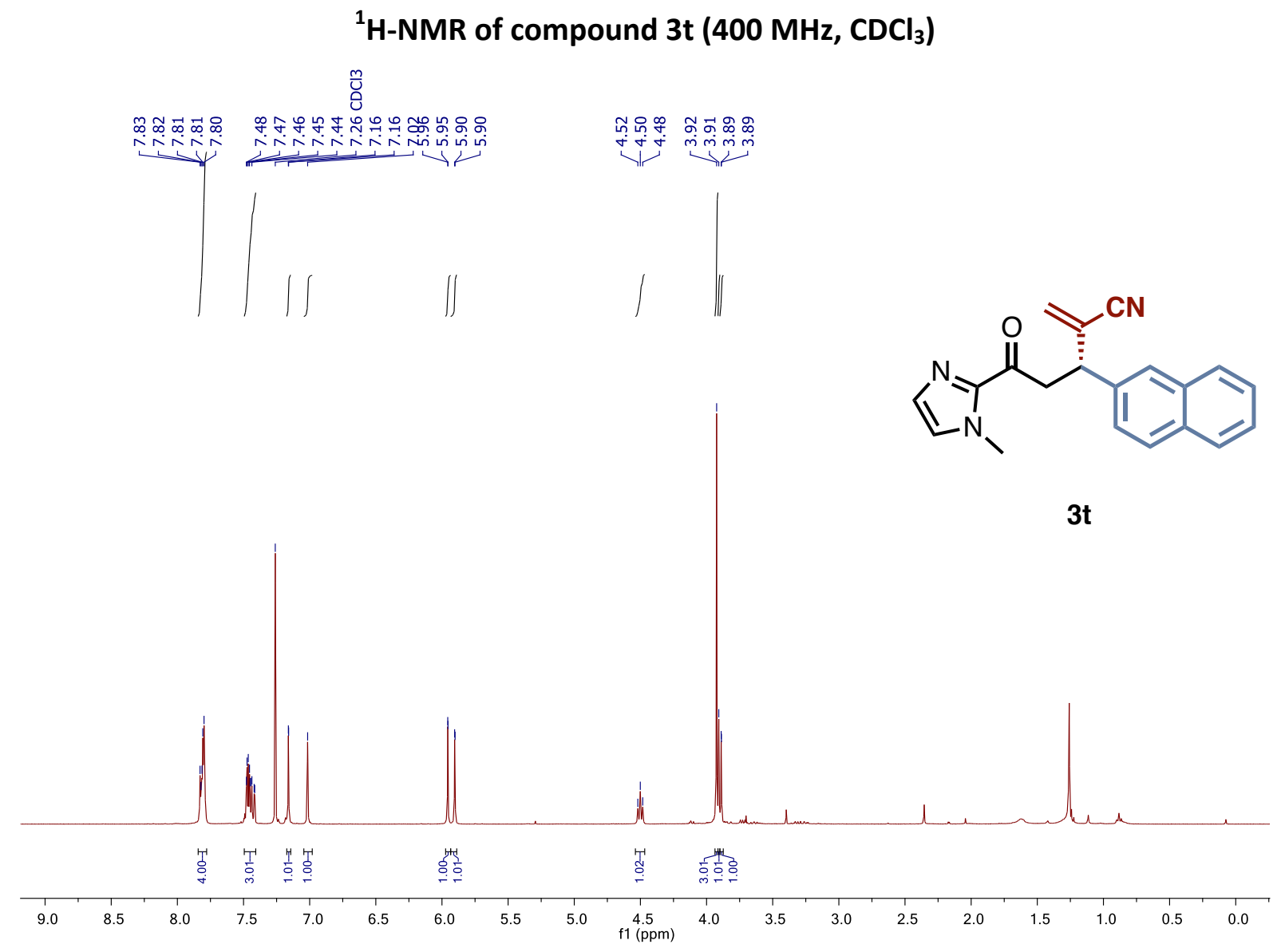

${ }^{13} \mathrm{C}-\mathrm{NMR}$ of compound $3 \mathrm{t}\left(101 \mathrm{MHz}, \mathrm{CDCl}_{3}\right)$

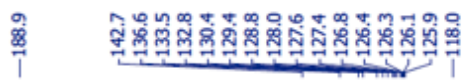

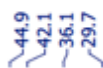

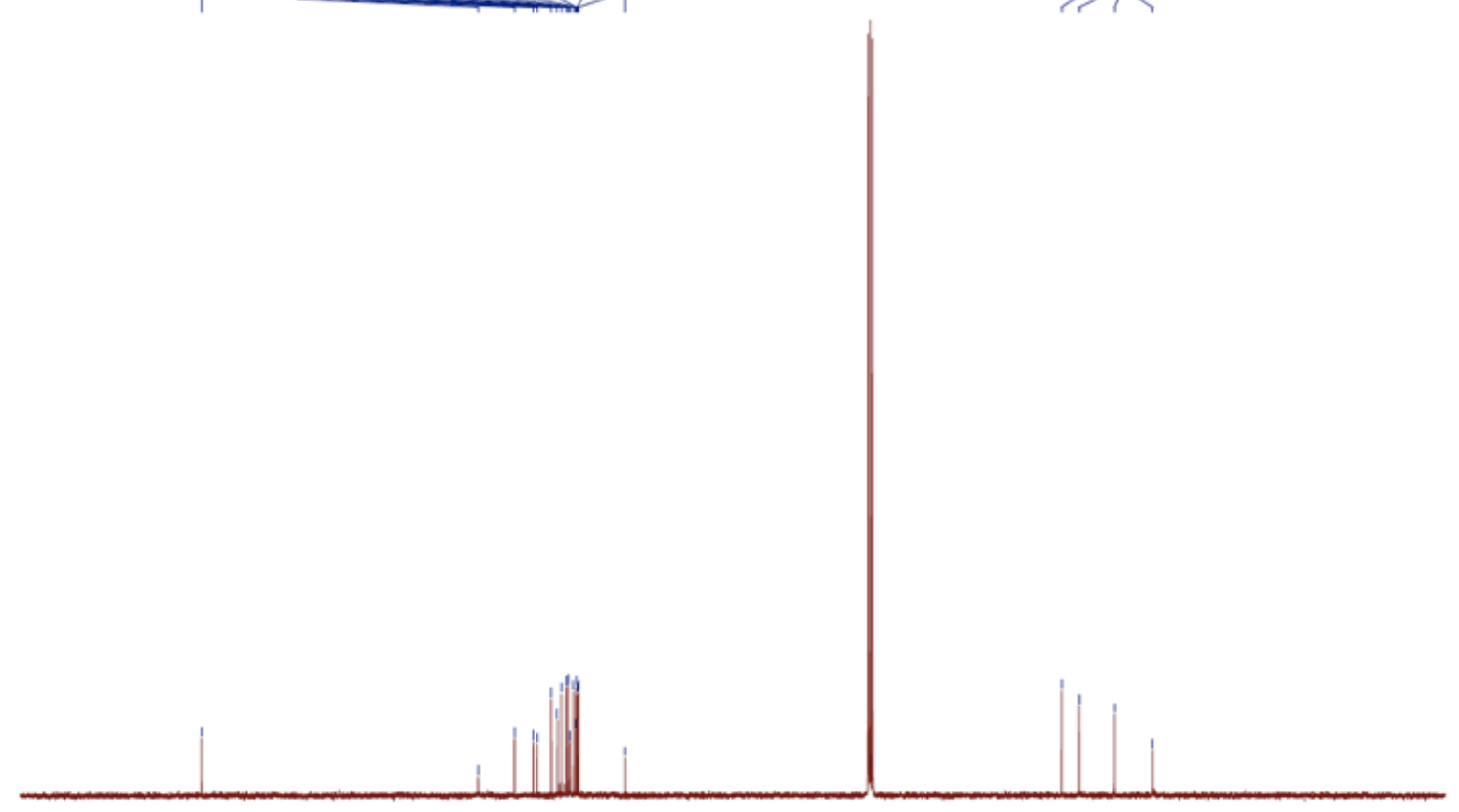

$\begin{array}{llllllllllllllllllllllllllllllll}210 & 200 & 190 & 180 & 170 & 160 & 150 & 140 & 130 & 120 & 110 & 100 & 90 & 80 & 70 & 60 & 50 & 40 & 30 & 20 & 10 & 0 & -10 & \end{array}$ 

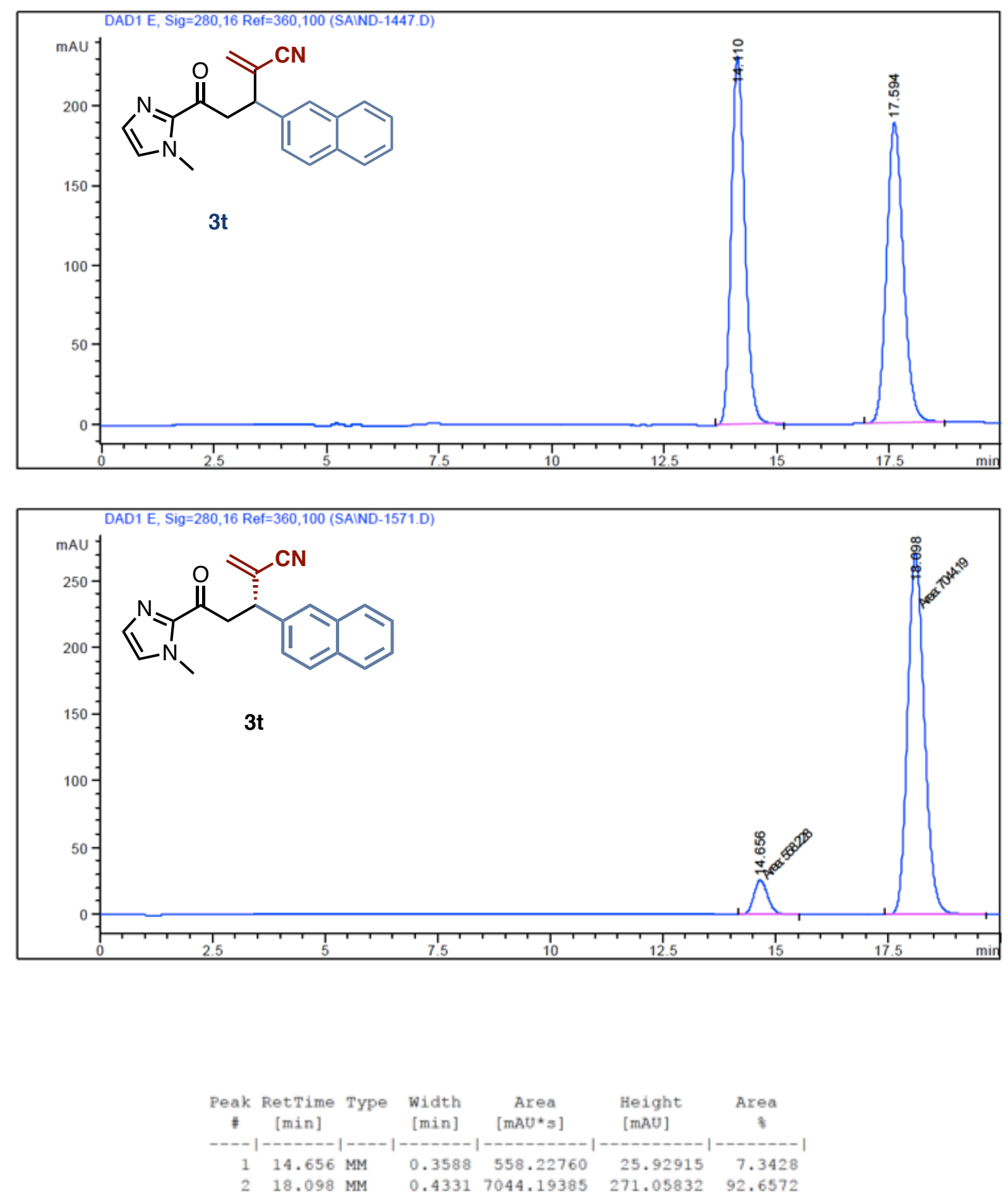


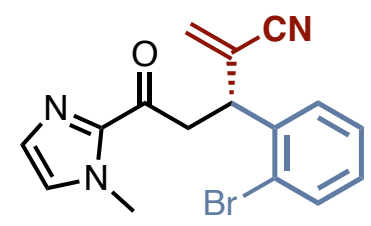

Molecular formula: $\mathrm{C}_{16} \mathrm{H}_{14} \mathrm{BrN}_{3} \mathrm{O}$

$\mathrm{MW}=343.21 \mathrm{~g} \cdot \mathrm{mol}^{-1}$

The title compound was isolated as a dark yellow oil (71 mg, $0.205 \mathrm{mmol}, 76 \%, 76 \%$ ee $)$ using general procedure F. Purified by flash column chromatography over silica gel $($ Hex/EtOAc/Acetone $=7: 2: 1)$.

${ }^{1} \mathrm{H}$ NMR $\left(400 \mathrm{MHz}, \mathrm{CDCl}_{3}\right) \delta 7.59(\mathrm{~d}, J=8.0,1.3 \mathrm{~Hz}, 1 \mathrm{H}), 7.42(\mathrm{dd}, J=7.8,1.7 \mathrm{~Hz}, 1 \mathrm{H}), 7.32$ $(\mathrm{td}, J=7.6,1.3 \mathrm{~Hz}, 1 \mathrm{H}), 7.15(\mathrm{~d}, J=1.0 \mathrm{~Hz}, 1 \mathrm{H}), 7.13(\mathrm{~m}, 1 \mathrm{H}), 7.04(\mathrm{~d}, J=0.8 \mathrm{~Hz}, 1 \mathrm{H}), 5.97$ $(\mathrm{d}, J=0.8 \mathrm{~Hz}, 1 \mathrm{H}), 5.92(\mathrm{~d}, J=1.3 \mathrm{~Hz}, 1 \mathrm{H}), 4.87(\mathrm{t}, J=7.4 \mathrm{~Hz}, 1 \mathrm{H}), 3.94(\mathrm{~s}, 3 \mathrm{H}), 3.88$ (dd, $J=17.7,8.2 \mathrm{~Hz}, 1 \mathrm{H}), 3.73(\mathrm{dd}, J=17.7,6.7 \mathrm{~Hz}, 1 \mathrm{H})$.

${ }^{13} \mathrm{C}$ NMR $\left(101 \mathrm{MHz}, \mathrm{CDCl}_{3}\right) \delta 188.5,142.6,138.2,133.4,131.7,129.4,129.1,128.5,128.1$, $126.0,127.4,124.9,124.5,117.6,43.3,41.8,36.1$.

HRMS (ESI) m/z: [M+Na] $]^{+}$calcd for $\mathrm{C}_{16} \mathrm{H}_{14} \mathrm{BrN}_{3} \mathrm{ONa} 366.0218$; found: 366.0229 .

IR $\left(\mathrm{cm}^{-1}\right):$ 2928, 2855, 2324, 1676, 1473, 1411, 1158.

$[a]_{D}^{20}=-40.3\left(c 2.23, \mathrm{CH}_{2} \mathrm{Cl}_{2}\right)$

HPLC analysis: carried out using a mixture of hexane and isopropyl alcohol (80:20 isocratic), at $1 \mathrm{~mL} \cdot \mathrm{min}^{-1}$, at $30{ }^{\circ} \mathrm{C}$ on an IC column, with UV detection $(\lambda=280 \mathrm{~nm})$. Retention times: 11.39 and $14.88 \mathrm{~min}$. 
${ }^{1} \mathrm{H}-\mathrm{NMR}$ of compound $3 \mathrm{u}\left(400 \mathrm{MHz}, \mathrm{CDCl}_{3}\right)$

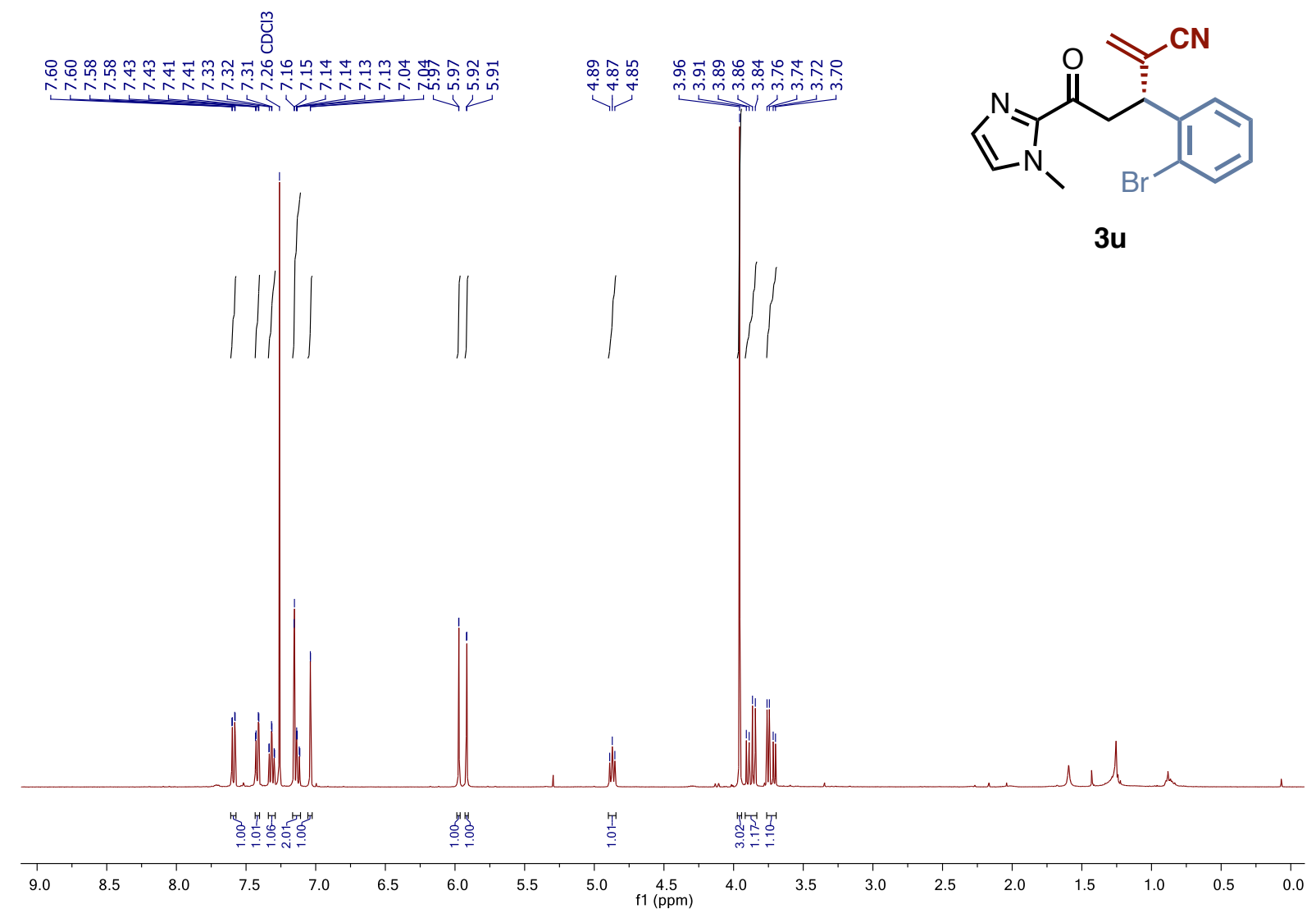

${ }^{13} \mathrm{C}-\mathrm{NMR}$ of compound $3 \mathrm{u}\left(101 \mathrm{MHz}, \mathrm{CDCl}_{3}\right.$ )

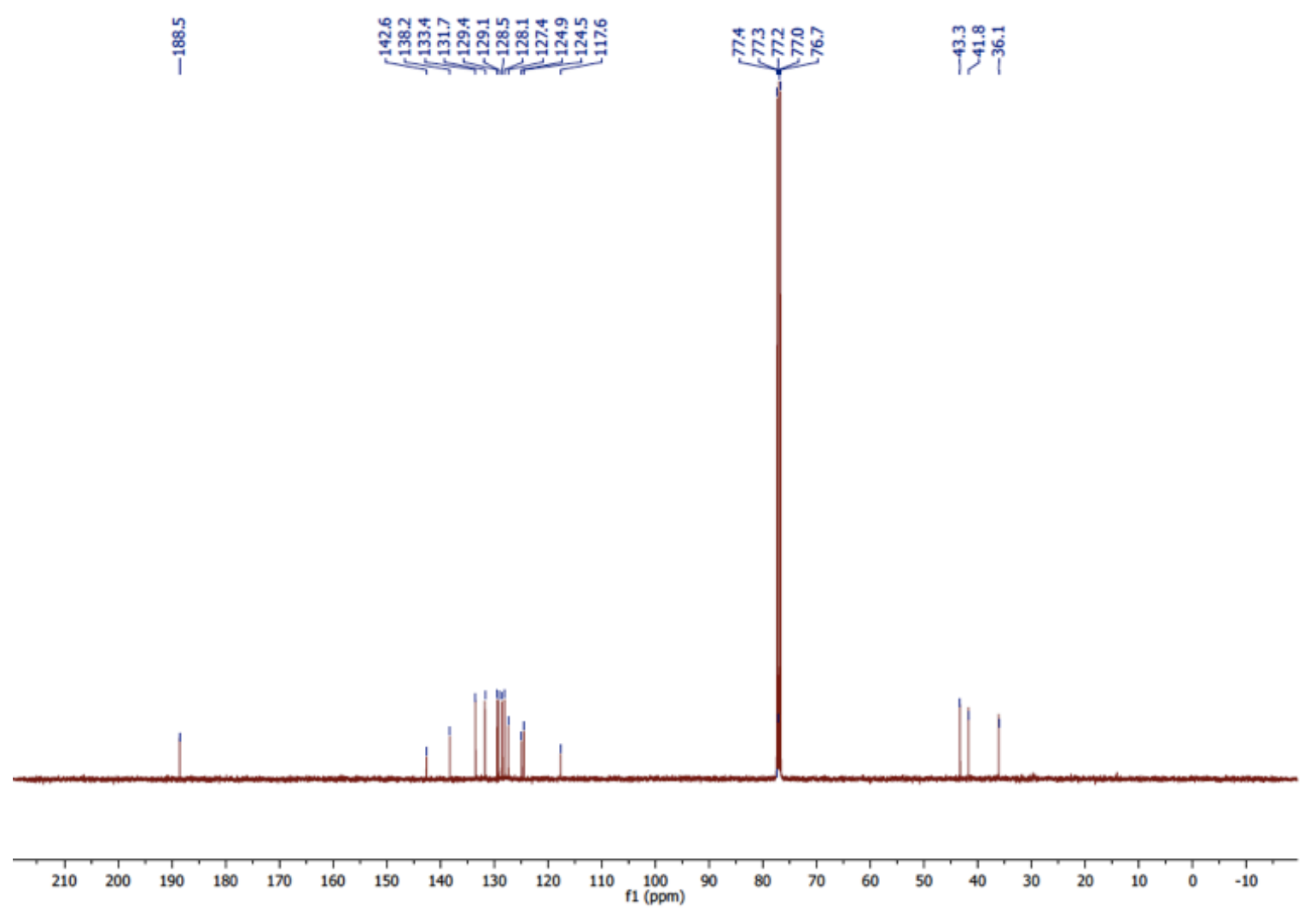



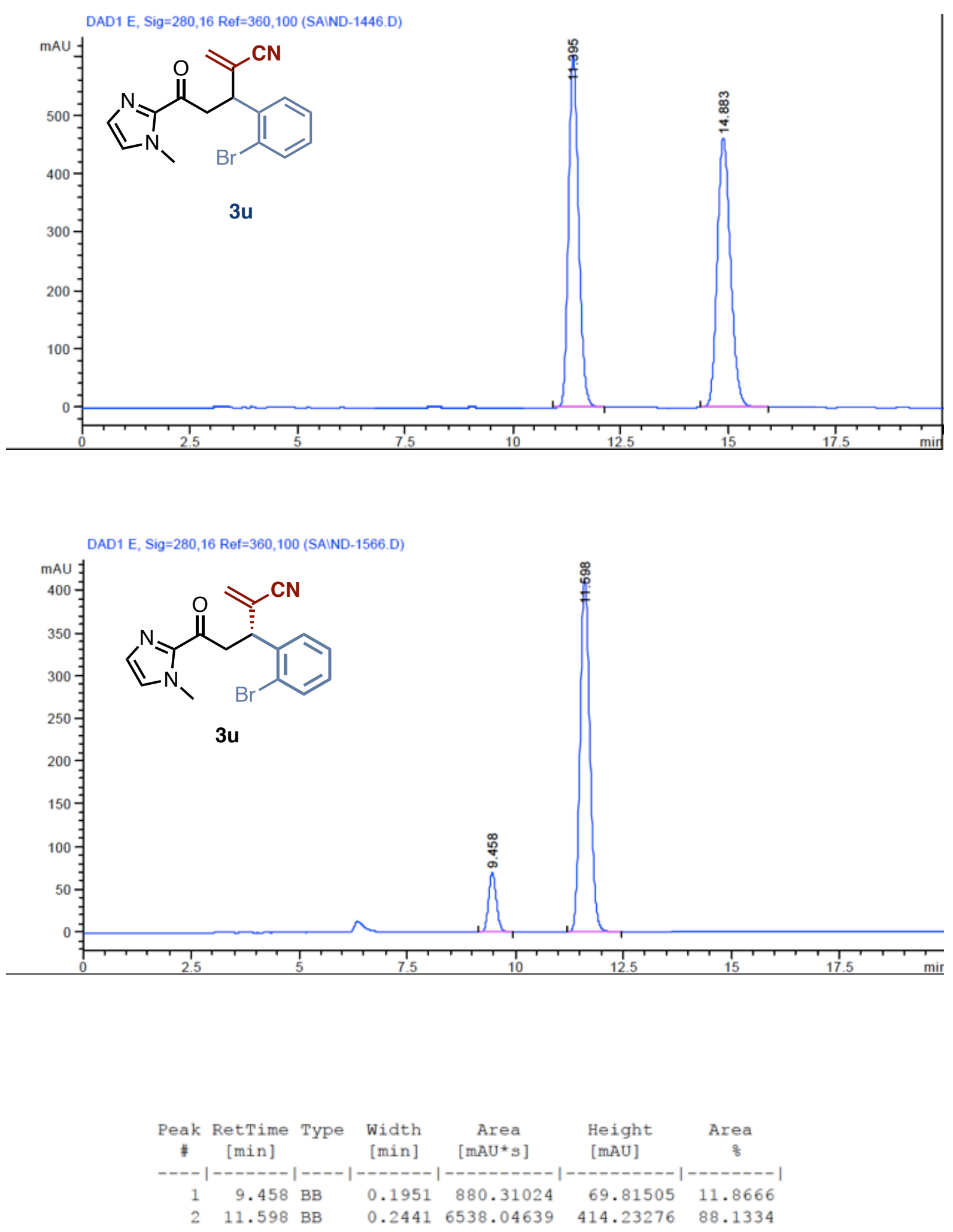
<smiles>C=C(C#N)[C@H](CC(=O)c1nccn1C)c1cccc(Br)c1</smiles>

Molecular formula: $\mathrm{C}_{16} \mathrm{H}_{14} \mathrm{~N}_{3} \mathrm{O}$

$\mathrm{MW}=344.21 \mathrm{~g} \cdot \mathrm{mol}^{-1}$

The title compound was isolated as a beige solid (64 mg, $0.184 \mathrm{mmol}, 68 \%, 76 \%$ ee) using general procedure F. Purified by flash column chromatography over silica gel $($ Hex/EtOAc/Acetone $=7: 2: 1)$.

${ }^{1} \mathrm{H}$ NMR $\left(400 \mathrm{MHz}, \mathrm{CDCl}_{3}\right) \delta 7.45(\mathrm{t}, J=1.9 \mathrm{~Hz}, 1 \mathrm{H}), 7.40(\mathrm{ddd}, J=7.8,2.0,1.2 \mathrm{~Hz}, 1 \mathrm{H}), 7.18$ $(\mathrm{dt}, J=7.9,1.6 \mathrm{~Hz}, 1 \mathrm{H}), 7.21(\mathrm{t}, J=7.8 \mathrm{~Hz}, 1 \mathrm{H}), 7.15(\mathrm{~d}, J=0.9 \mathrm{~Hz}, 1 \mathrm{H}), 7.04(\mathrm{~d}, J=0.9 \mathrm{~Hz}, 1 \mathrm{H})$, $5.95(\mathrm{~d}, J=0.8 \mathrm{~Hz}, 1 \mathrm{H}), 5.88(\mathrm{~d}, J=1.3 \mathrm{~Hz}, 1 \mathrm{H}), 4.28(\mathrm{t}, J=7.4 \mathrm{~Hz}, 1 \mathrm{H}), 3.95(\mathrm{~s}, 3 \mathrm{H}), 3.80$ (dd, $J=17.4,7.6 \mathrm{~Hz}, 1 \mathrm{H}), 3.71(\mathrm{dd}, J=17.4,7.2 \mathrm{~Hz}, 1 \mathrm{H})$.

${ }^{13} \mathrm{C}$ NMR $\left(101 \mathrm{MHz}, \mathrm{CDCl}_{3}\right) \delta 188.5,142.5,141.6,131.0,130.9,130.7,130.5,129.5,127.5$, $126.5,125.7,122.9,117.7,44.5,42.5,36.1$.

HRMS (ESI) m/z: [M+H] $]^{+}$calcd for $\mathrm{C}_{16} \mathrm{H}_{15} \mathrm{BrN}_{3} \mathrm{O}$ 344.0398; found: 344.0407 .

IR $\left(\mathrm{cm}^{-1}\right):$ 2957, 2926, 2855, 2225, 1678, 1477, 1410, 1231, 1012.

$[a]_{D}^{20}=-6.8\left(c 2.0, \mathrm{CH}_{2} \mathrm{Cl}_{2}\right)$

HPLC analysis: carried out using a mixture of hexane and isopropyl alcohol (80:20 isocratic), at $1 \mathrm{~mL} \cdot \mathrm{min}^{-1}$, at $30{ }^{\circ} \mathrm{C}$ on an IC column, with UV detection $(\lambda=280 \mathrm{~nm})$. Retention times: 10.49 and $12.67 \mathrm{~min}$. 
${ }^{1} \mathrm{H}-\mathrm{NMR}$ of compound $3 \mathrm{v}\left(400 \mathrm{MHz}, \mathrm{CDCl}_{3}\right)$

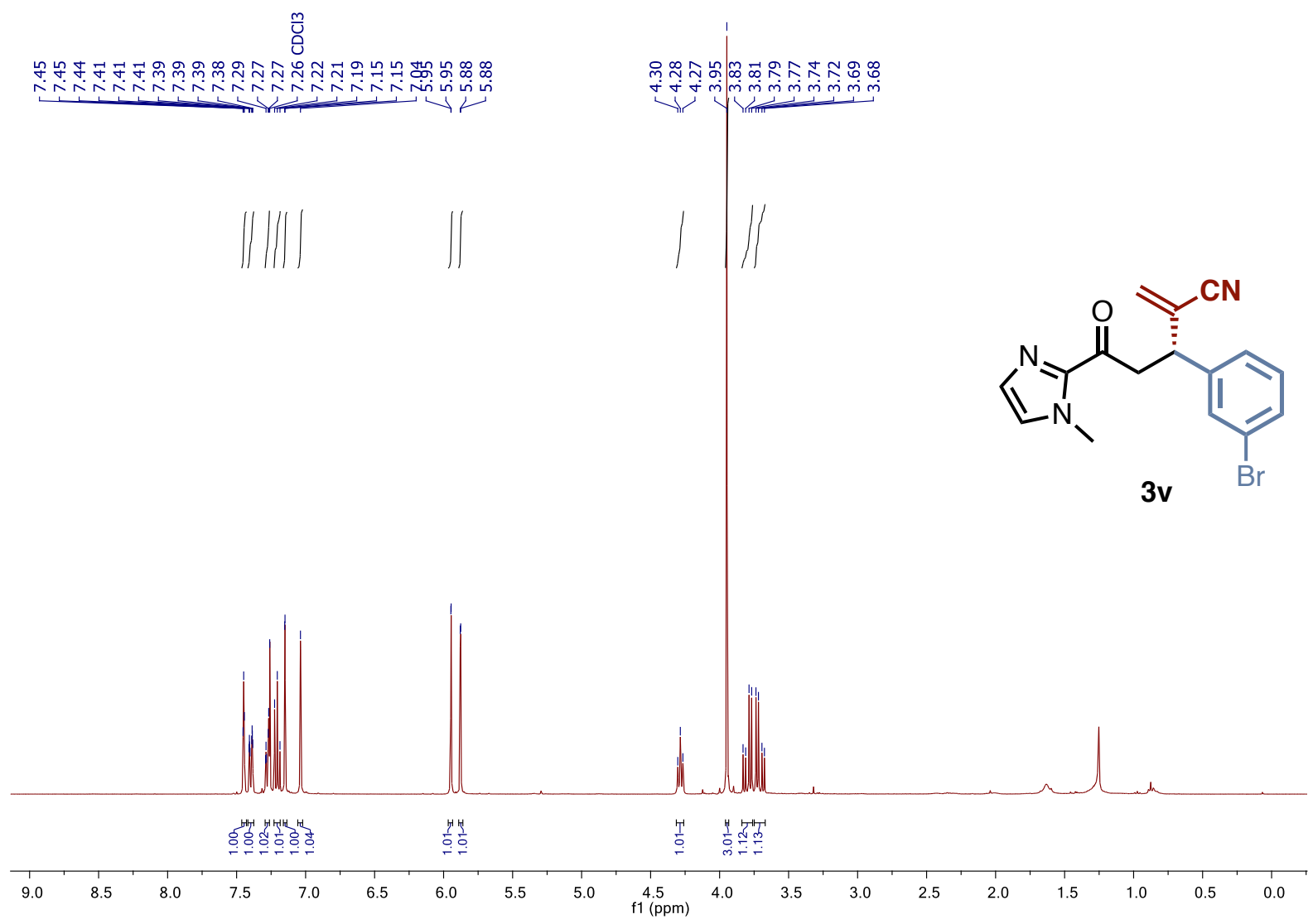

${ }^{13} \mathrm{C}-\mathrm{NMR}$ of compound $3 \mathrm{v}\left(101 \mathrm{MHz}, \mathrm{CDCl}_{3}\right)$

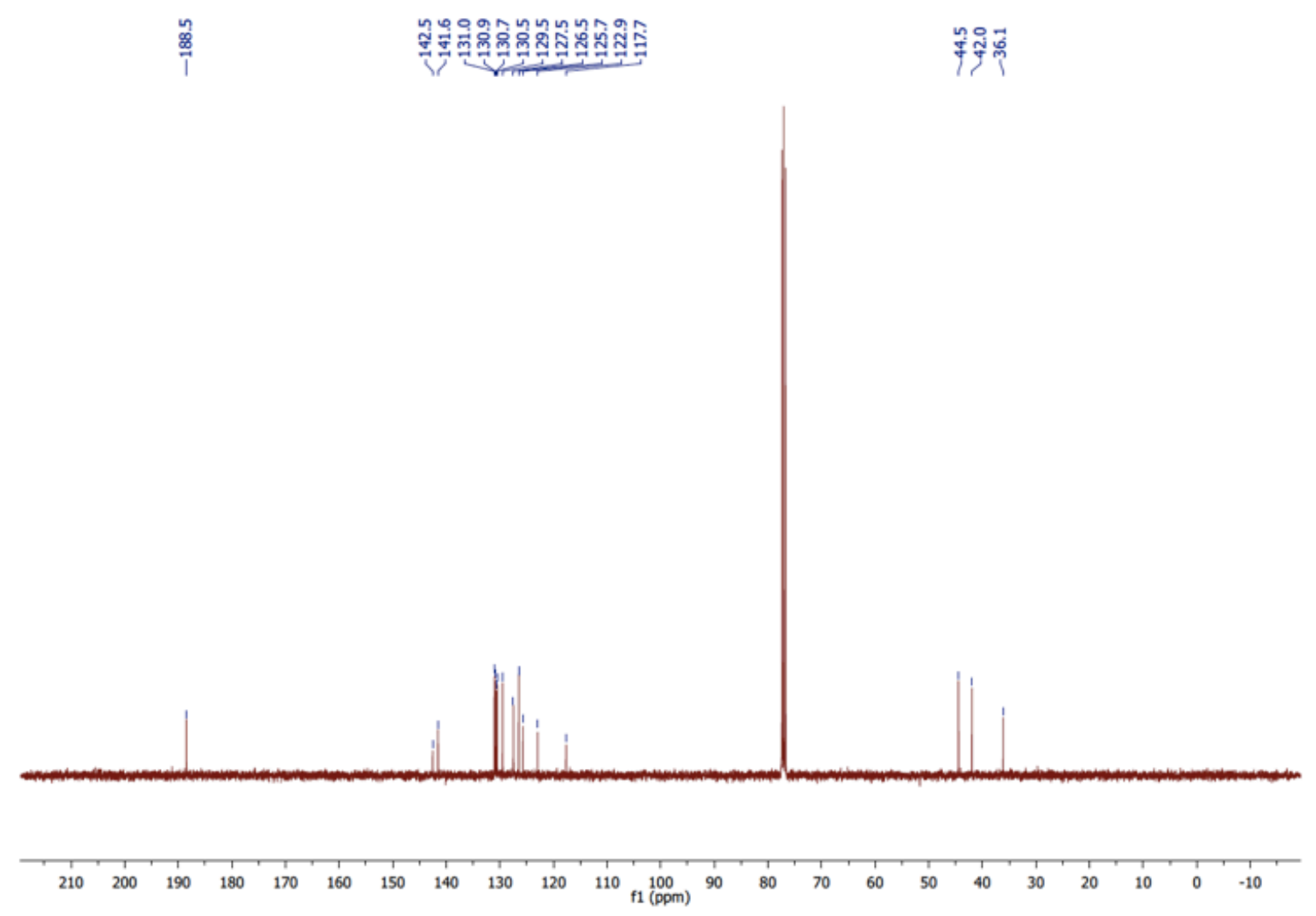



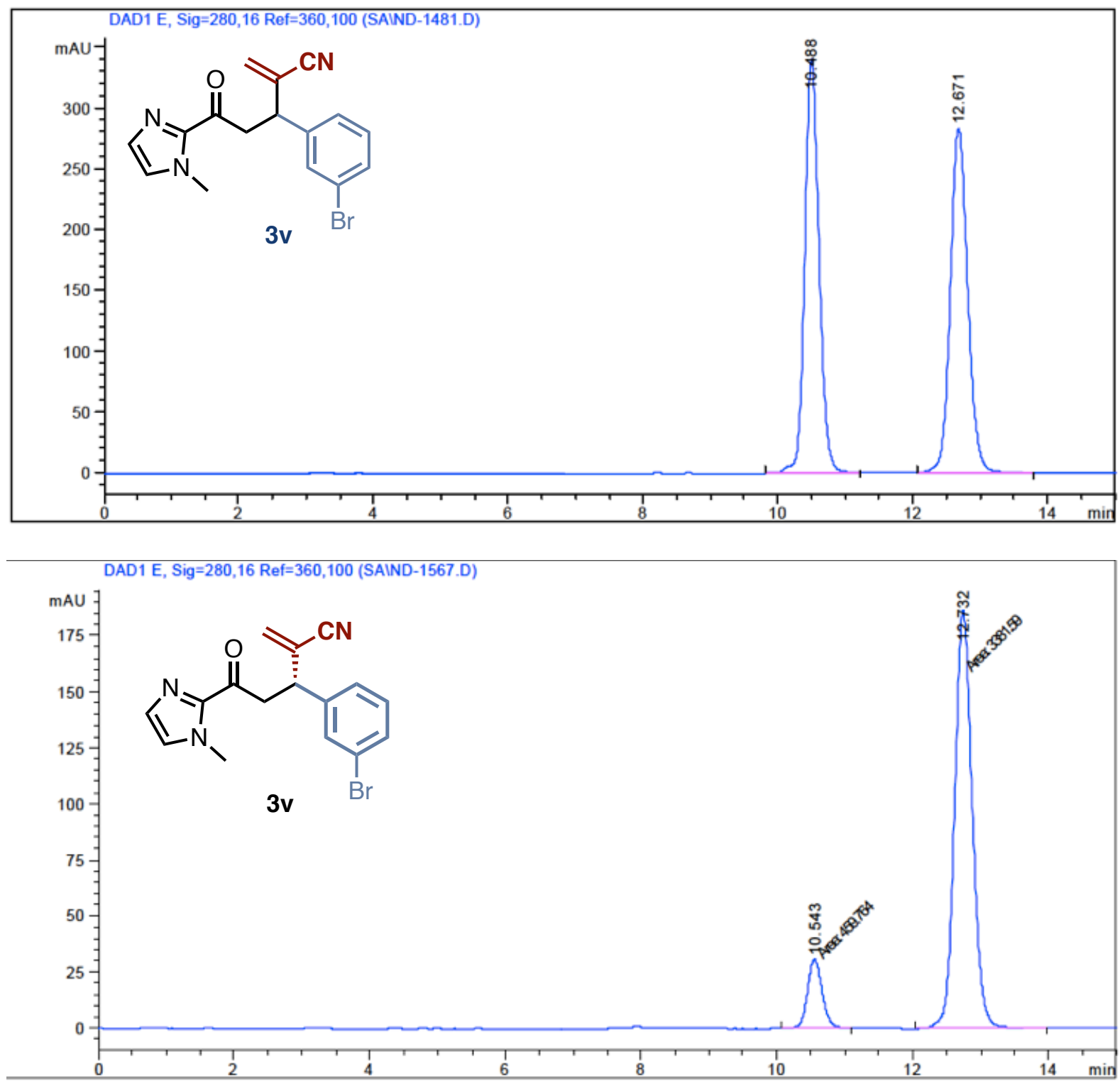

\begin{tabular}{|c|c|c|c|c|c|c|}
\hline $\begin{array}{c}\text { Peak } \\
\ddagger\end{array}$ & $\begin{array}{c}\text { RetTime } \\
\text { [min] }\end{array}$ & Type & $\begin{array}{l}\text { width } \\
\text { [min] }\end{array}$ & $\begin{array}{c}\text { Area } \\
{\left[\mathrm{mAU}^{*} \mathrm{~S}\right]}\end{array}$ & $\begin{array}{l}\text { Height } \\
\text { [mAU] }\end{array}$ & $\begin{array}{c}\text { Area } \\
\frac{9}{5}\end{array}$ \\
\hline-- & 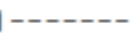 & & ----- & -------- & $----\infty---\mid$ & -------1 \\
\hline 1 & 10.543 & MM & 0.2493 & 459.76416 & 30.73665 & 11.9688 \\
\hline 2 & 12.732 & $\mathrm{MM}$ & 0.3022 & 3381.59180 & 186.50507 & 88.0312 \\
\hline
\end{tabular}




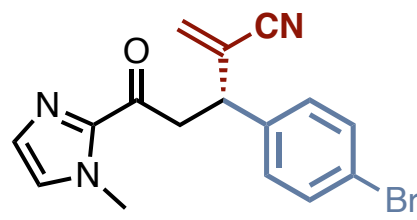

Molecular formula: $\mathrm{C}_{16} \mathrm{H}_{14} \mathrm{~N}_{3} \mathrm{O}$

$\mathrm{MW}=344.21 \mathrm{~g} \cdot \mathrm{mol}^{-1}$

The title compound was isolated as a clear oil (55 mg, $0.159 \mathrm{mmol}, 59 \%, 77 \%$ ee) using general procedure F. Purified by flash column chromatography over silica gel $($ Hex/EtOAc/Acetone $=7: 2: 1)$.

${ }^{1}$ H NMR (400 MHz, $\left.\mathrm{CDCl}_{3}\right) \delta 7.45(\mathrm{~m}, 2 \mathrm{H}), 7.20(\mathrm{~m}, 2 \mathrm{H}), 7.15(\mathrm{~d}, J=1.0 \mathrm{~Hz}, 1 \mathrm{H}), 7.03$ (d, $J=0.9 \mathrm{~Hz}, 1 \mathrm{H}), 5.93(\mathrm{~d}, J=0.9 \mathrm{~Hz}, 1 \mathrm{H}), 5.85(\mathrm{~d}, J=1.4 \mathrm{~Hz}, 1 \mathrm{H}), 4.29(\mathrm{t}, J=7.5 \mathrm{~Hz}, 1 \mathrm{H}), 3.94(\mathrm{~s}$, $3 \mathrm{H}), 3.78$ (dd, $J=14.5,4.6 \mathrm{~Hz}, 1 \mathrm{H}), 3.72(\mathrm{dd}, J=14.5,4.7 \mathrm{~Hz}, 1 \mathrm{H}$ ).

${ }^{13} \mathrm{C}$ NMR $\left(101 \mathrm{MHz}, \mathrm{CDCl}_{3}\right) \delta 188.6,142.6,138.2,132.1,130.4,129.6,129.5,127.5,126.0$, $121.7,117.7,44.4,41.9,36.1$.

HRMS (ESI) m/z: [M+Na] $]^{+}$calcd for $\mathrm{C}_{16} \mathrm{H}_{14} \mathrm{BrN}_{3} \mathrm{ONa} 366.0218$; found: 344.0229 .

IR $\left(\mathrm{cm}^{-1}\right):$ 3110, 2961, 2926, 2224, 1674, 1492, 1406, 1292, 1158

$[\boldsymbol{a}]_{D}^{20}=-16.8\left(c 0.83, \mathrm{CH}_{2} \mathrm{Cl}_{2}\right)$

HPLC analysis: carried out using a mixture of hexane and isopropyl alcohol (80:20 isocratic) at $1 \mathrm{~mL} \cdot \mathrm{min}^{-1}$, at $30{ }^{\circ} \mathrm{C}$ on an IC column, with UV detection $(\lambda=280 \mathrm{~nm})$. Retention times: 9.59 and $11.92 \mathrm{~min}$. 
${ }^{1} \mathrm{H}-\mathrm{NMR}$ of compound $3 \mathrm{w}\left(400 \mathrm{MHz}, \mathrm{CDCl}_{3}\right)$

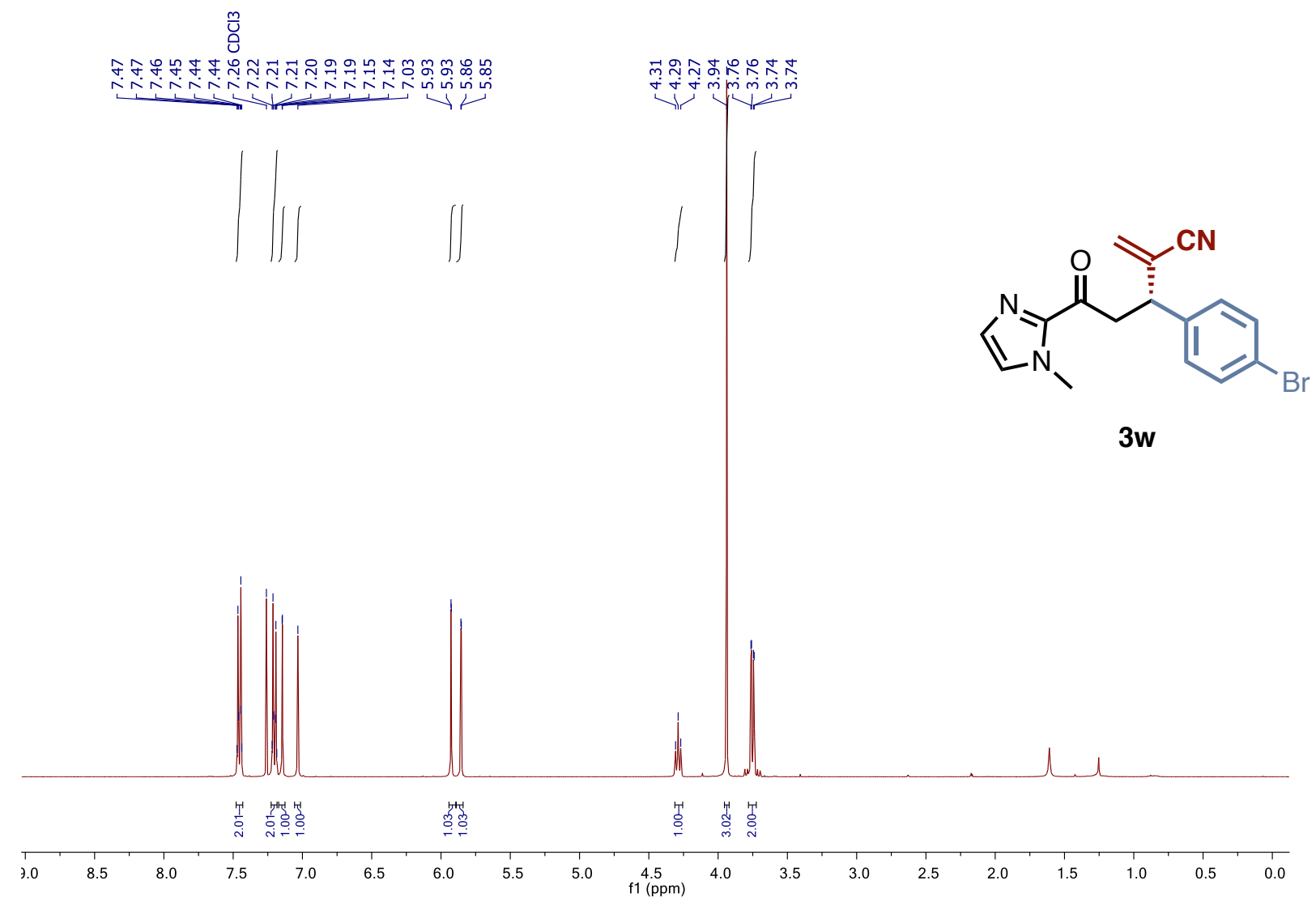

${ }^{13} \mathrm{C}-\mathrm{NMR}$ of compound 3w (101 $\left.\mathrm{MHz}, \mathrm{CDCl}_{3}\right)$

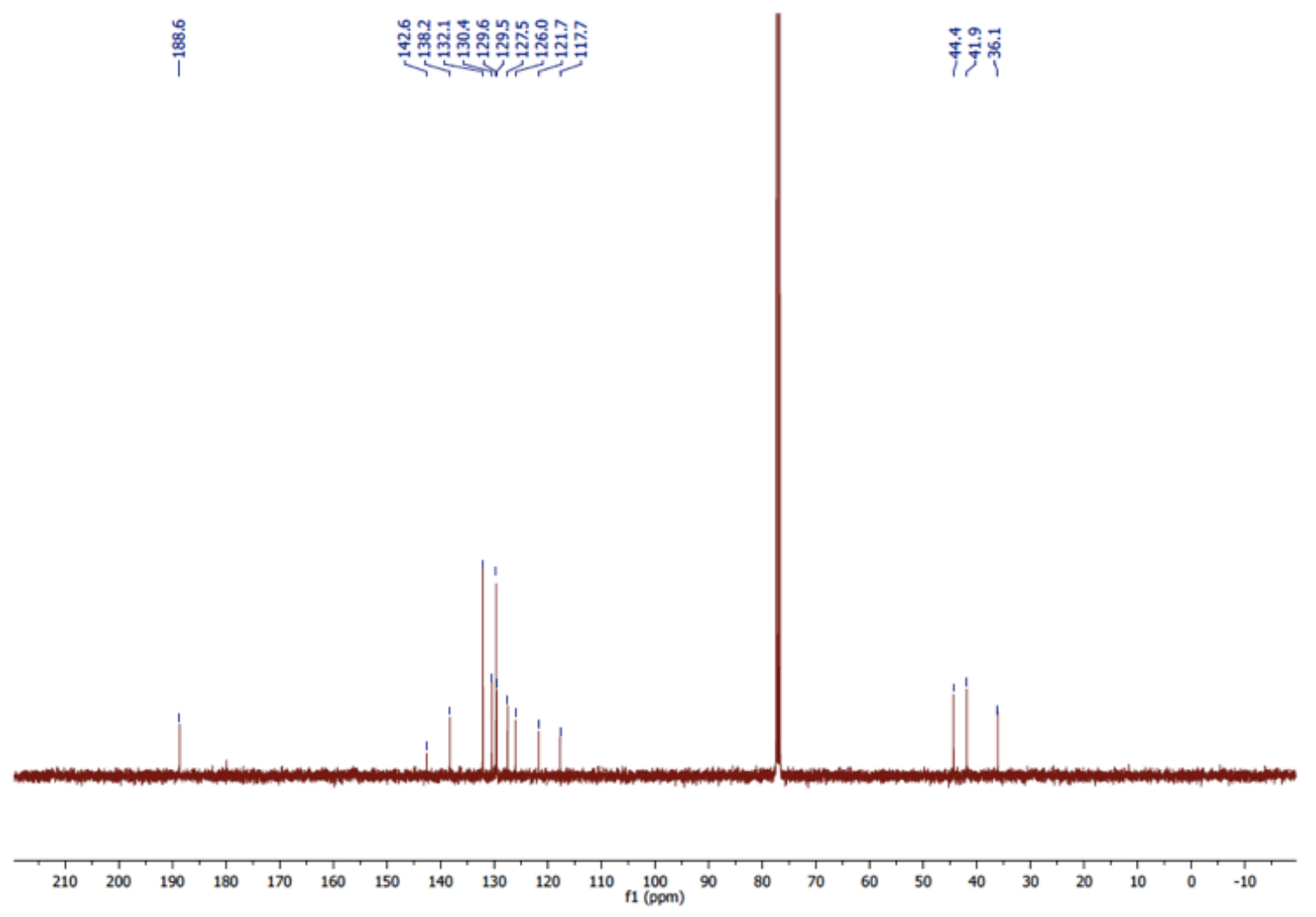



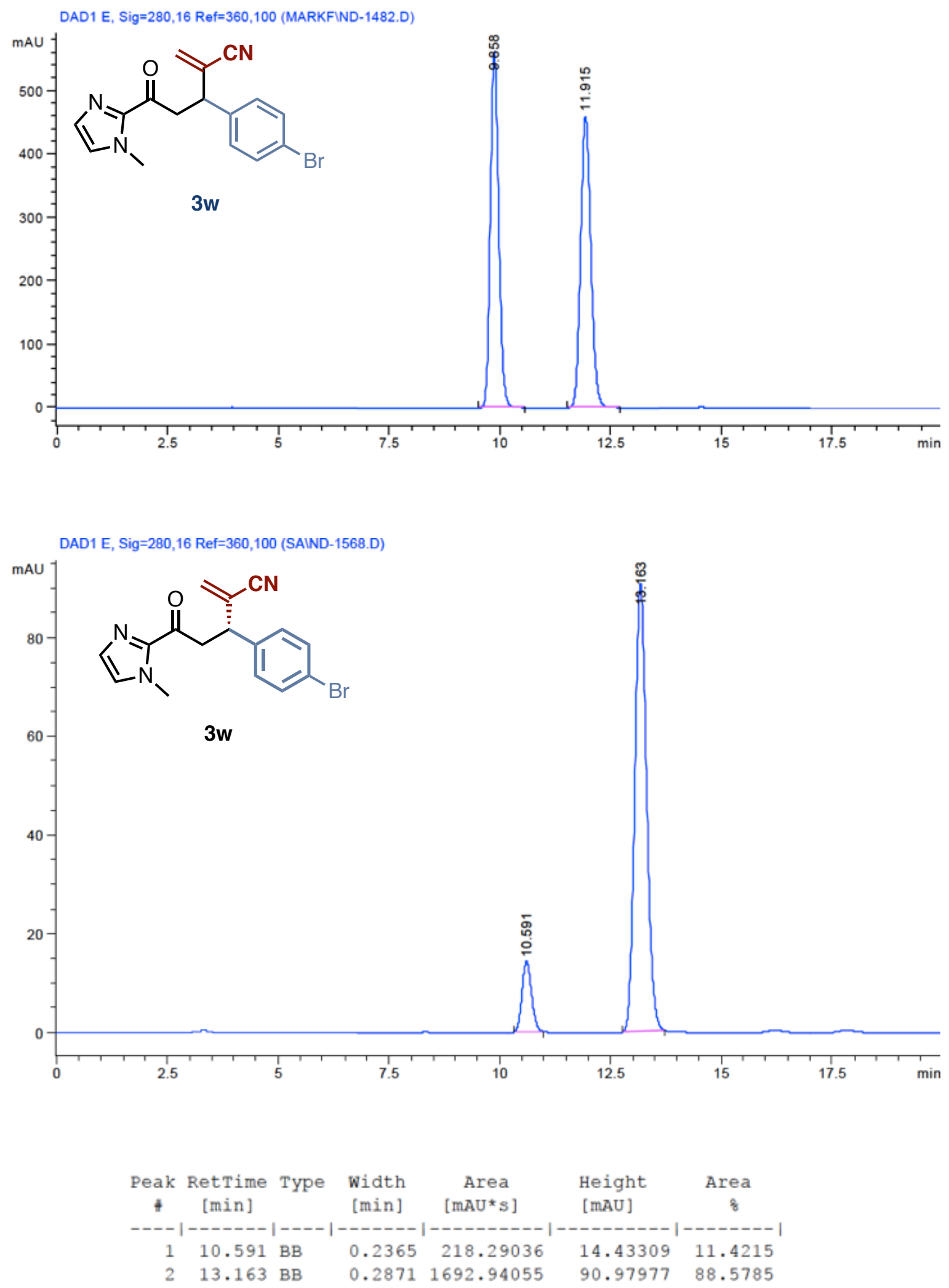


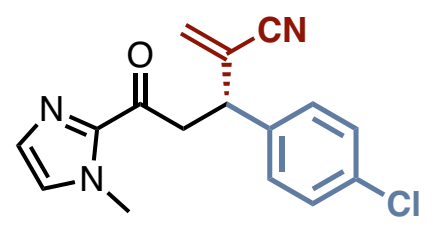

Molecular formula: $\mathrm{C}_{16} \mathrm{H}_{14} \mathrm{ClN}_{3} \mathrm{O}$

$$
\mathrm{MW}=299.76 \mathrm{~g} \cdot \mathrm{mol}^{-1}
$$

The title compound was isolated as an amorphous solid (44 mg, $0.149 \mathrm{mmol}, 54 \%, 70 \%$ ee) using general procedure F. Purified by flash column chromatography over silica gel $($ Hex/EtOAc/Acetone $=7: 2: 1)$.

${ }^{1}$ H NMR $\left(400 \mathrm{MHz}, \mathrm{CDCl}_{3}\right) \delta 7.32-7.24(\mathrm{~m}, 4 \mathrm{H}), 7.15(\mathrm{~d}, J=0.9 \mathrm{~Hz}, 1 \mathrm{H}), 7.03(\mathrm{~d}, J=0.9 \mathrm{~Hz}$, 1H), $5.93(\mathrm{~d}, J=1.1 \mathrm{~Hz}, 1 \mathrm{H}), 5.86(\mathrm{~d}, J=1.1 \mathrm{~Hz}, 1 \mathrm{H}), 4.30(\mathrm{dd}, J=7.6,7.3 \mathrm{~Hz}, 1 \mathrm{H}), 3.94(\mathrm{~s}, 3 \mathrm{H})$, $3.76(\mathrm{~d}, J=7.3 \mathrm{~Hz}, 1 \mathrm{H}), 3.75(\mathrm{~d}, J=7.6 \mathrm{~Hz}, 1 \mathrm{H})$.

${ }^{13} \mathrm{C}$ NMR $\left(101 \mathrm{MHz}, \mathrm{CDCl}_{3}\right) \delta 188.7,142.7,137.8,133.7,130.5,129.6,129.4(2 \mathrm{C}), 129.2(2 \mathrm{C})$, $127.6,126.2,117.8,44.4,42.1,36.2$.

HRMS (ESI) m/z: [M+H] calcd for $\mathrm{C}_{16} \mathrm{H}_{15} \mathrm{CIN}_{3} \mathrm{O}$ 300.0904; found: 300.0919 .

IR $\left(\mathrm{cm}^{-1}\right):$ 3110, 2957, 2926, 2224, 1674, 1493, 1408, 1292, 1156, 1093.

$[\boldsymbol{a}]_{\boldsymbol{D}}^{20}=-36.0\left(c 0.67, \mathrm{CH}_{2} \mathrm{Cl}_{2}\right)$

HPLC analysis: carried out using a mixture of hexane and isopropyl alcohol (80:20 isocratic) at $25{ }^{\circ} \mathrm{C}$ on an IC column, with UV detection $(\lambda=280 \mathrm{~nm})$. Retention times: 9.6 and 12.1 $\min$. 
${ }^{1} \mathrm{H}-\mathrm{NMR}$ of compound $3 \times\left(400 \mathrm{MHz}, \mathrm{CDCl}_{3}\right)$

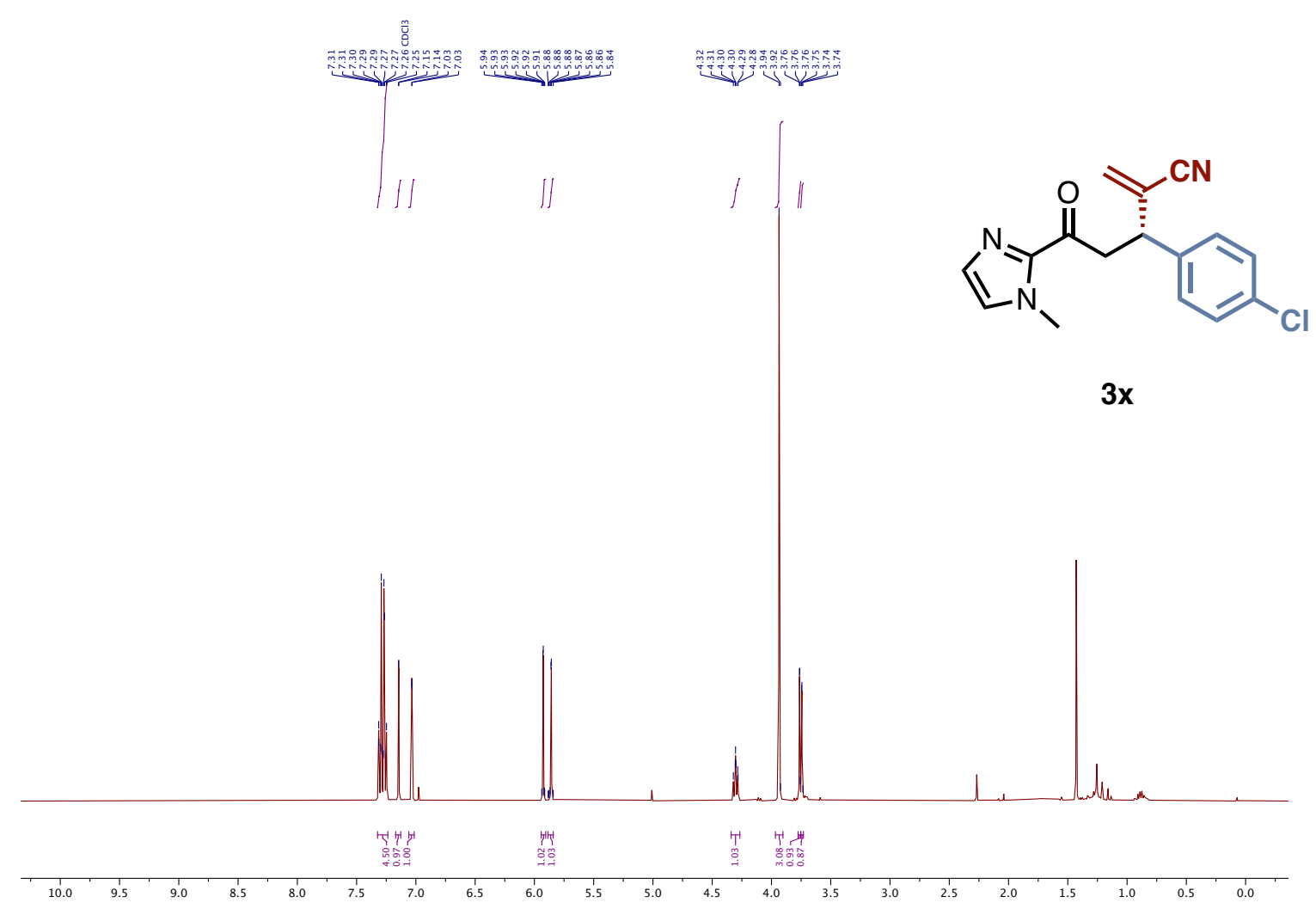

${ }^{13} \mathrm{C}-\mathrm{NMR}$ of compound $3 \times\left(101 \mathrm{MHz}, \mathrm{CDCl}_{3}\right.$ )

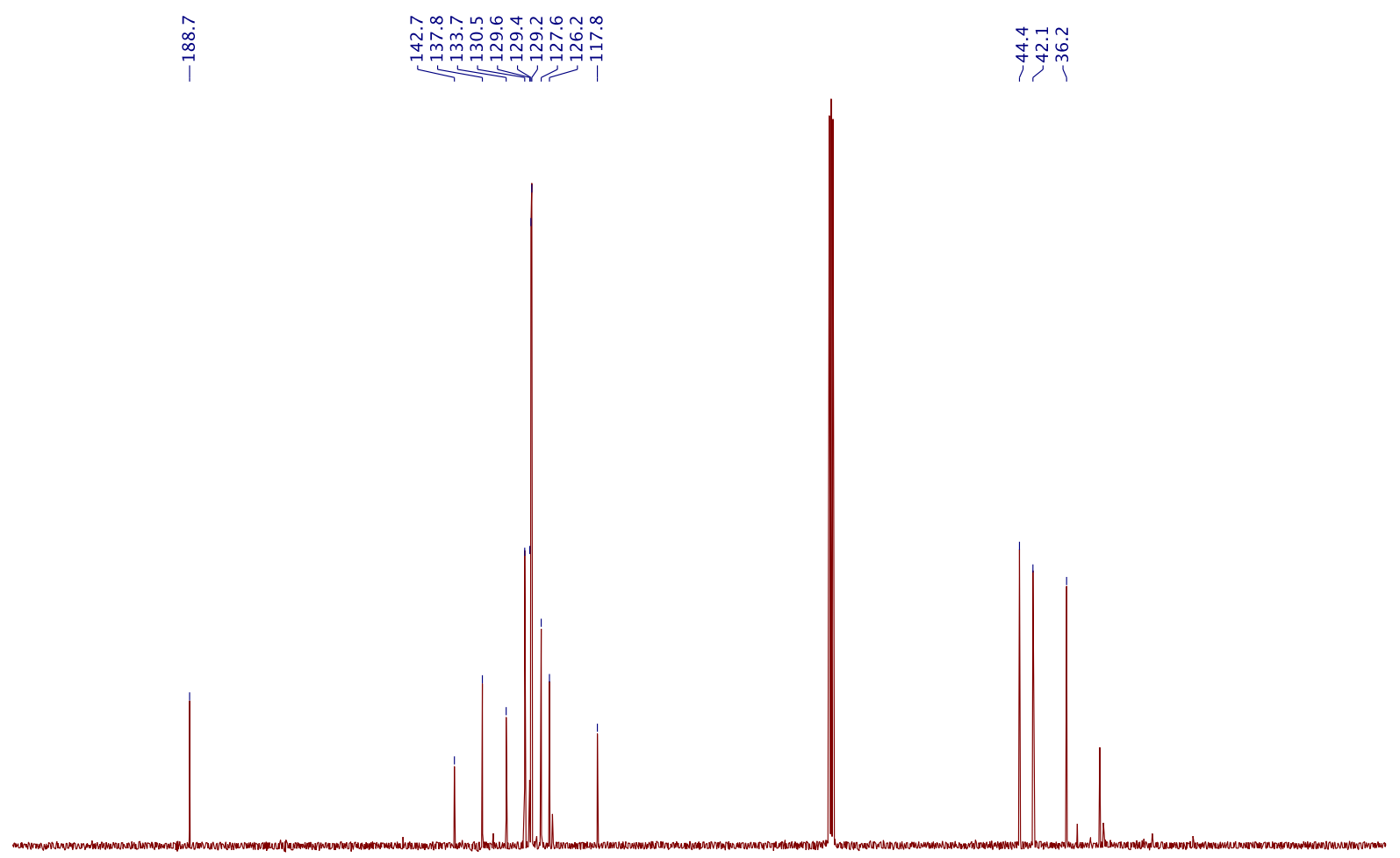

$\begin{array}{lllllllllllllllllllllll}210 & 200 & 190 & 180 & 170 & 160 & 150 & 140 & 130 & 120 & 110 & 100 & 90 & 80 & 70 & 60 & 50 & 40 & 30 & 20 & 10 & 0 & -10\end{array}$ 

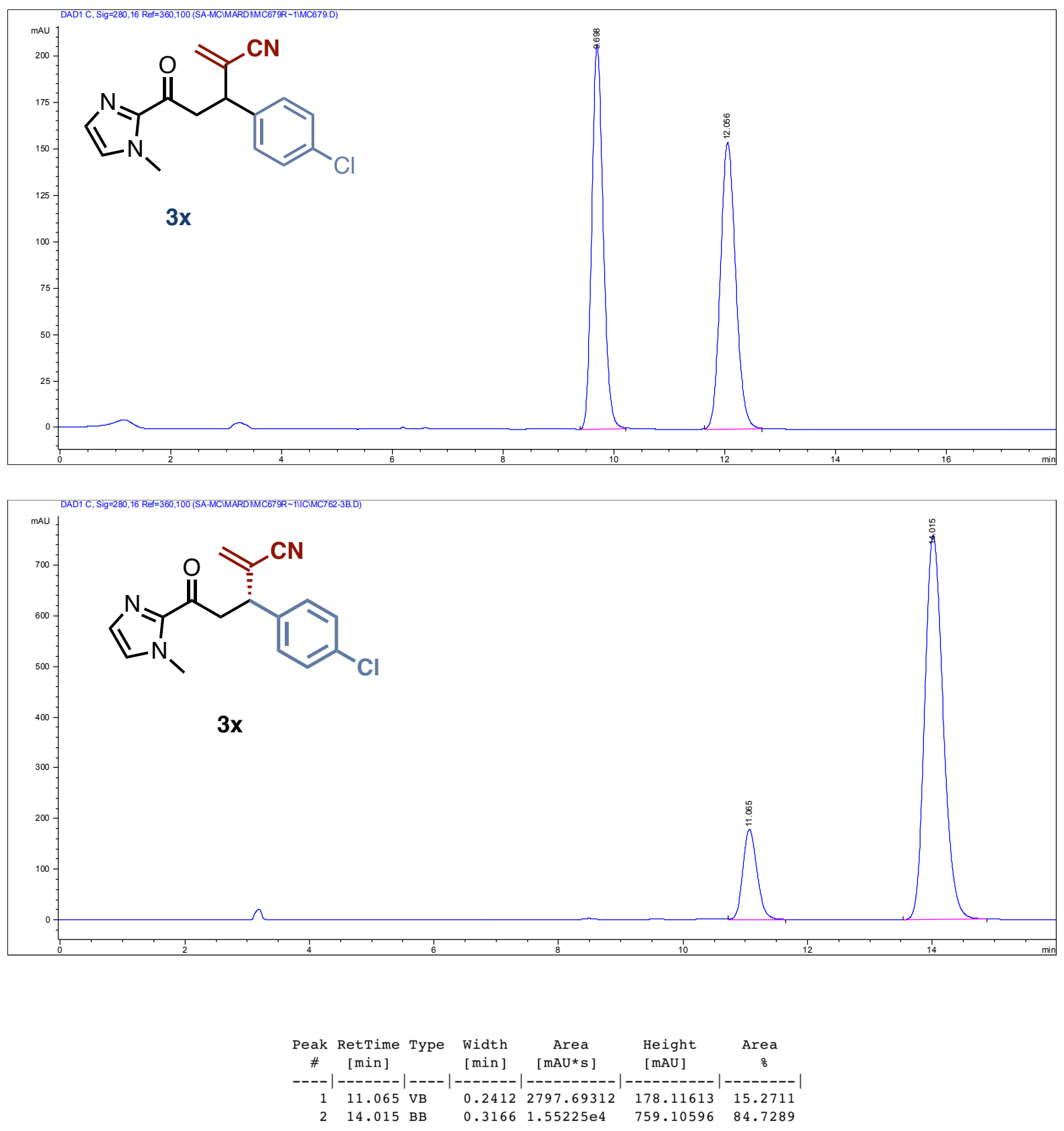


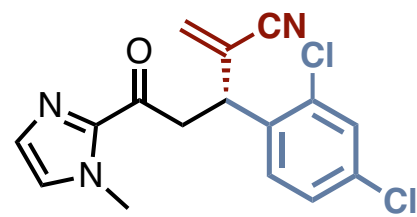

Molecular formula: $\mathrm{C}_{16} \mathrm{H}_{13} \mathrm{Cl}_{2} \mathrm{~N}_{3} \mathrm{O}$

$\mathrm{MW}=334.20 \mathrm{~g} \cdot \mathrm{mol}^{-1}$

The title compound was isolated as a yellow oil $(70 \mathrm{mg}, 0.209 \mathrm{mmol}, 63 \%, 76 \%$ ee $)$ using general procedure F. Purified by flash column chromatography over silica gel $($ Hex/EtOAc/Acetone $=7: 2: 1)$.

${ }^{1} \mathrm{H}$ NMR $\left(400 \mathrm{MHz}, \mathrm{CDCl}_{3}\right) \delta 7.42(\mathrm{~d}, J=2.2 \mathrm{~Hz}, 1 \mathrm{H}), 7.36(\mathrm{~d}, J=8.4 \mathrm{~Hz}, 1 \mathrm{H}), 7.25(\mathrm{dd}, J=8.3$, $2.2 \mathrm{~Hz}, 1 \mathrm{H}), 7.16(\mathrm{~d}, J=0.6 \mathrm{~Hz}, 1 \mathrm{H}), 7.05(\mathrm{~s}, 1 \mathrm{H}), 5.97$ (d, J=0.7 Hz, 1H), 5.91 (d, J=1.2 Hz, $1 \mathrm{H}), 4.82(\mathrm{t}, J=7.5 \mathrm{~Hz}, 1 \mathrm{H}), 3.95(\mathrm{~s}, 3 \mathrm{H}), 3.79(\mathrm{dd}, J=16.1,7.4 \mathrm{~Hz}, 2 \mathrm{H})$.

${ }^{13} \mathrm{C}$ NMR $\left(101 \mathrm{MHz}, \mathrm{CDCl}_{3}\right) \delta 188.4,142.6,135.4,135.0,134.2,131.9,130.1,129.7,129.4$, $127.9,127.7,124.2,117.5,41.5,40.6,36.3$.

HRMS (ESI) m/z: [M+Na] $]^{+}$calcd for $\mathrm{C}_{16} \mathrm{H}_{13} \mathrm{Cl}_{2} \mathrm{~N}_{3} \mathrm{ONa} 356.00333$; Found: 356.0323.

IR $\left(\mathrm{cm}^{-1}\right):$ 3110, 2957, 2341, 2363, 2224, 1737, 1676, 1588, 1475, 1408, 1290, 1158.

$[a]_{D}^{20}=-45.8\left(\right.$ c 2.17, $\left.\mathrm{CH}_{2} \mathrm{Cl}_{2}\right)$

HPLC analysis: carried out using a mixture of hexane and isopropyl alcohol (80:20 isocratic), at $1 \mathrm{~mL} \cdot \mathrm{min}^{-1}$, at $30{ }^{\circ} \mathrm{C}$ on an IC column, with UV detection $(\lambda=280 \mathrm{~nm})$. Retention times: 7.44 and $8.31 \mathrm{~min}$. 
${ }^{1} \mathrm{H}-\mathrm{NMR}$ of compound $3 \mathrm{y}\left(400 \mathrm{MHz}^{\mathrm{CDCl}}{ }_{3}\right)$

范

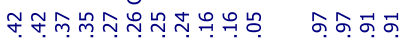

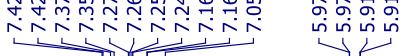

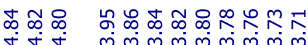

F mmmmimm m

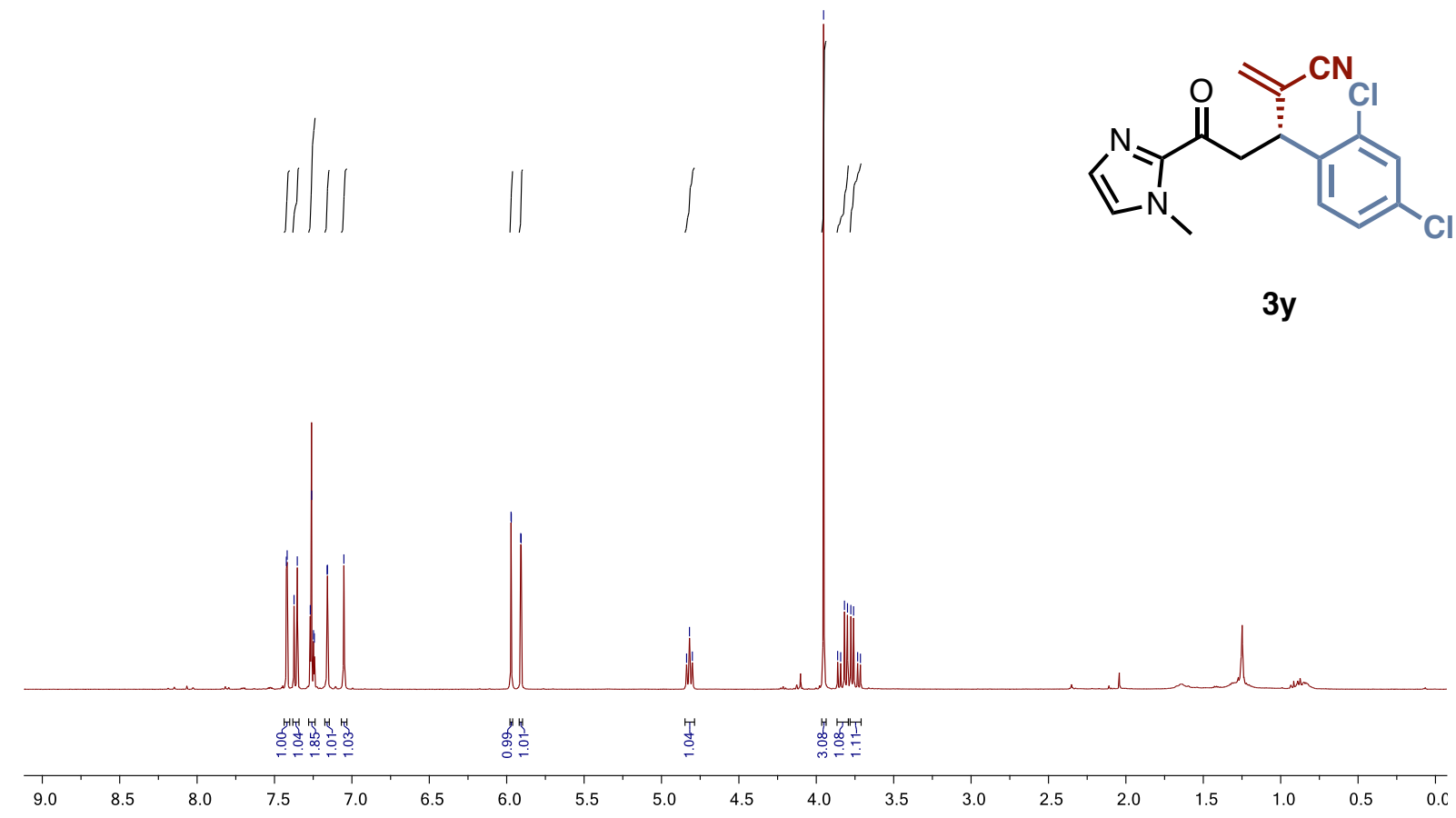

${ }^{13} \mathrm{C}-\mathrm{NMR}$ of compound 3y (101 $\left.\mathrm{MHz}, \mathrm{CDCl}_{3}\right)$

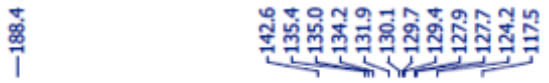

锩曷

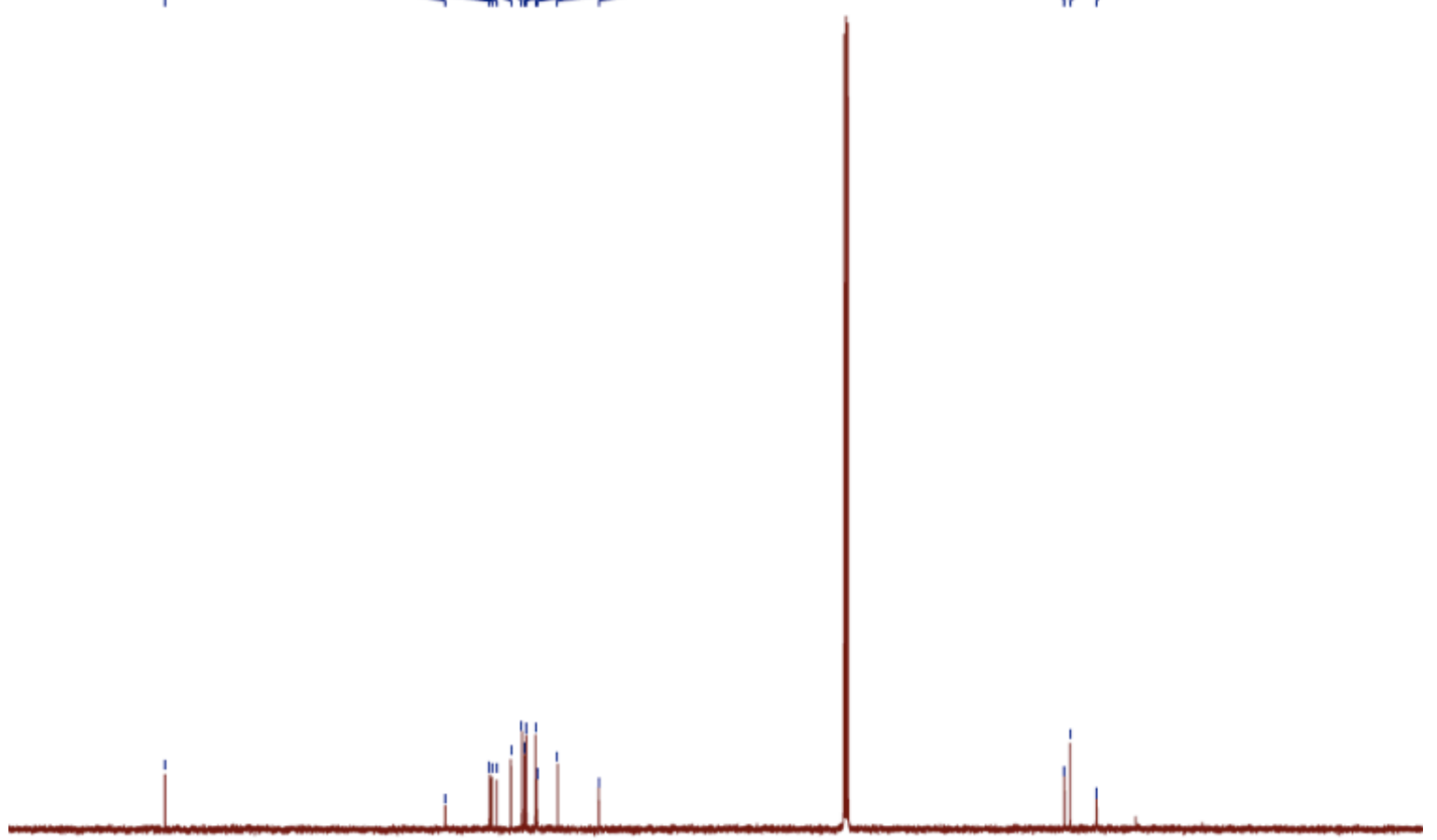

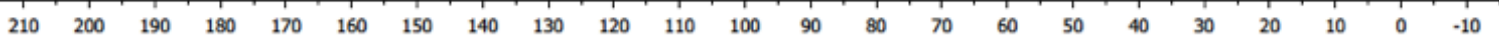



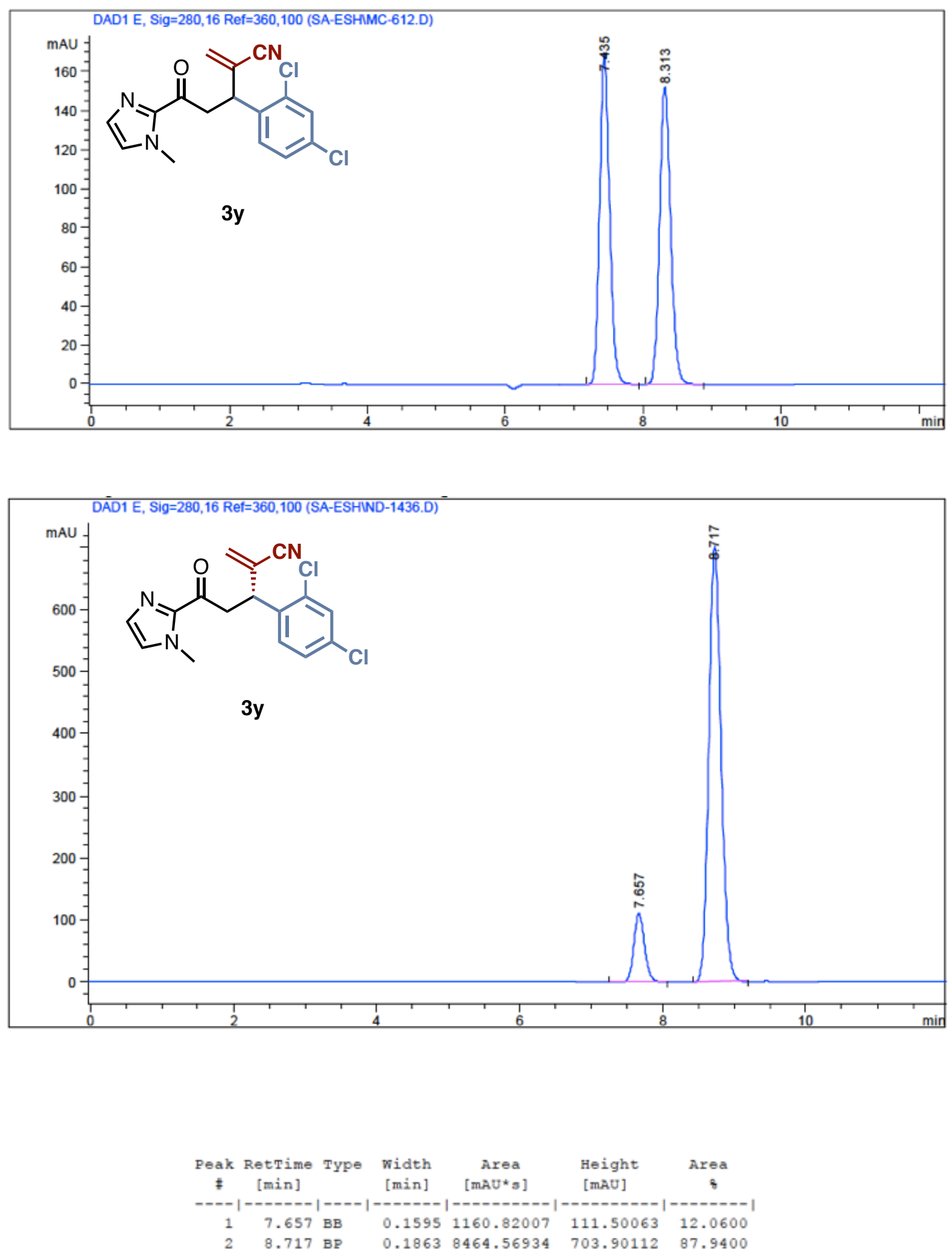

nitrile (3z)

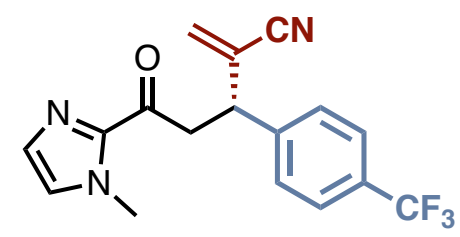

Molecular formula: $\mathrm{C}_{17} \mathrm{H}_{14} \mathrm{~N}_{3} \mathrm{~F}_{3} \mathrm{O}$

$\mathbf{M W}=333.31 \mathrm{~g} \cdot \mathrm{mol}^{-1}$

The title compound was isolated as a yellow solid (43 mg, $0.130 \mathrm{mmol}, 48 \%, 78 \%$ ee $)$ using general procedure F. Purified by flash column chromatography over silica gel $($ Hex/EtOAc/Acetone $=6: 3: 1)$.

${ }^{1}$ H NMR $\left(400 \mathrm{MHz}, \mathrm{CDCl}_{3}\right) \delta 7.60(\mathrm{~d}, J=7.9 \mathrm{~Hz}, 2 \mathrm{H}), 7.46(\mathrm{~d}, J=8.0 \mathrm{~Hz}, 2 \mathrm{H}), 7.16(\mathrm{~s}, 1 \mathrm{H}), 7.05$ (s, 1H), $5.96(\mathrm{~d}, J=0.9 \mathrm{~Hz}, 1 \mathrm{H}), 5.90(\mathrm{~d}, J=1.3 \mathrm{~Hz}, 1 \mathrm{H}), 4.39(\mathrm{t}, J=7.3 \mathrm{~Hz}, 1 \mathrm{H}), 3.94(\mathrm{~s}, 3 \mathrm{H})$, $3.78(\mathrm{~m}, 1 \mathrm{H})$.

${ }^{13} \mathrm{C}$ NMR $\left(101 \mathrm{MHz}, \mathrm{CDCl}_{3}\right) \delta 188.4,143.2,130.8,130.2,129.8,129.5,128.3,125.9$ (q, $J=3.8 \mathrm{~Hz}), 125.6,125.3,44.7,41.8,36.1$.

${ }^{19}$ F NMR $\left(377 \mathrm{MHz}, \mathrm{CDCl}_{3}\right) \delta-62.6(\mathrm{~s}, 3 \mathrm{~F})$.

HRMS (ESI) m/z: [M+H] $]^{+}$calcd for $\mathrm{C}_{17} \mathrm{H}_{15} \mathrm{~N}_{3} \mathrm{~F}_{3} \mathrm{O}_{3}$ 334.1167; found: 334.1146 .

IR $\left(\mathrm{cm}^{-1}\right):$ 2959, 2928, 2224, 1680, 1413, 1328, 1168, 1117, 1073.

$[\boldsymbol{a}]_{\boldsymbol{D}}^{20}=-17.0\left(\mathrm{c} 1.47, \mathrm{CH}_{2} \mathrm{Cl}_{2}\right)$

HPLC analysis: carried out using a mixture of hexane and isopropyl alcohol (80:20 isocratic), at $1 \mathrm{~mL} \cdot \mathrm{min}^{-1}$, at $30{ }^{\circ} \mathrm{C}$ on an IC column, with UV detection $(\lambda=280 \mathrm{~nm})$. Retention times: 7.31 and $9.04 \mathrm{~min}$. 
${ }^{1} \mathrm{H}-\mathrm{NMR}$ of compound $3 \mathrm{z}\left(400 \mathrm{MHz}, \mathrm{CDCl}_{3}\right.$ )

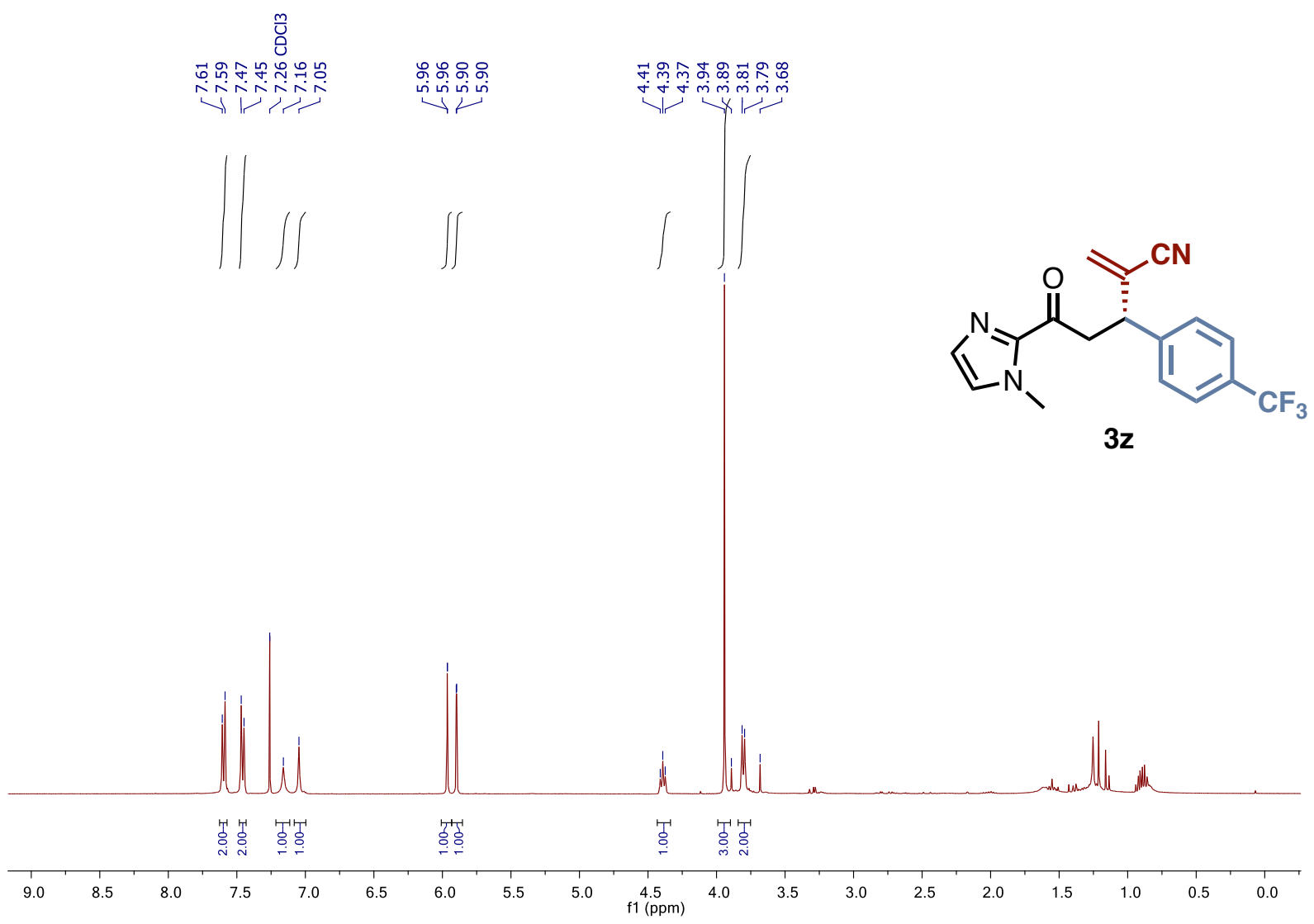

${ }^{13} \mathrm{C}-\mathrm{NMR}$ of compound $3 \mathrm{z}\left(101 \mathrm{MHz}, \mathrm{CDCl}_{3}\right)$

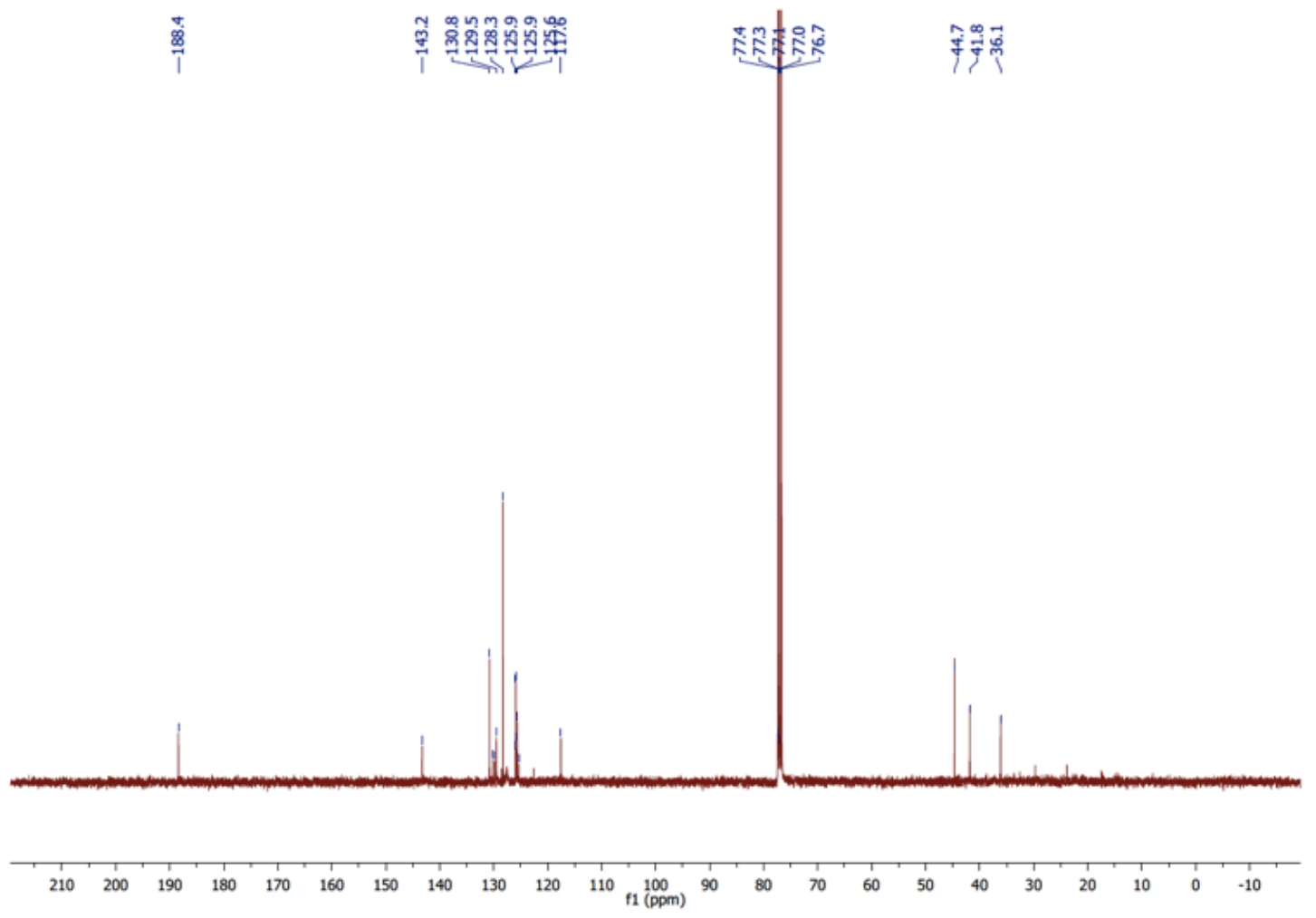


${ }^{19}$ F-NMR of compound $3 z$ (377 MHz, $\mathrm{CDCl}_{3}$ )

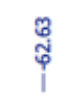

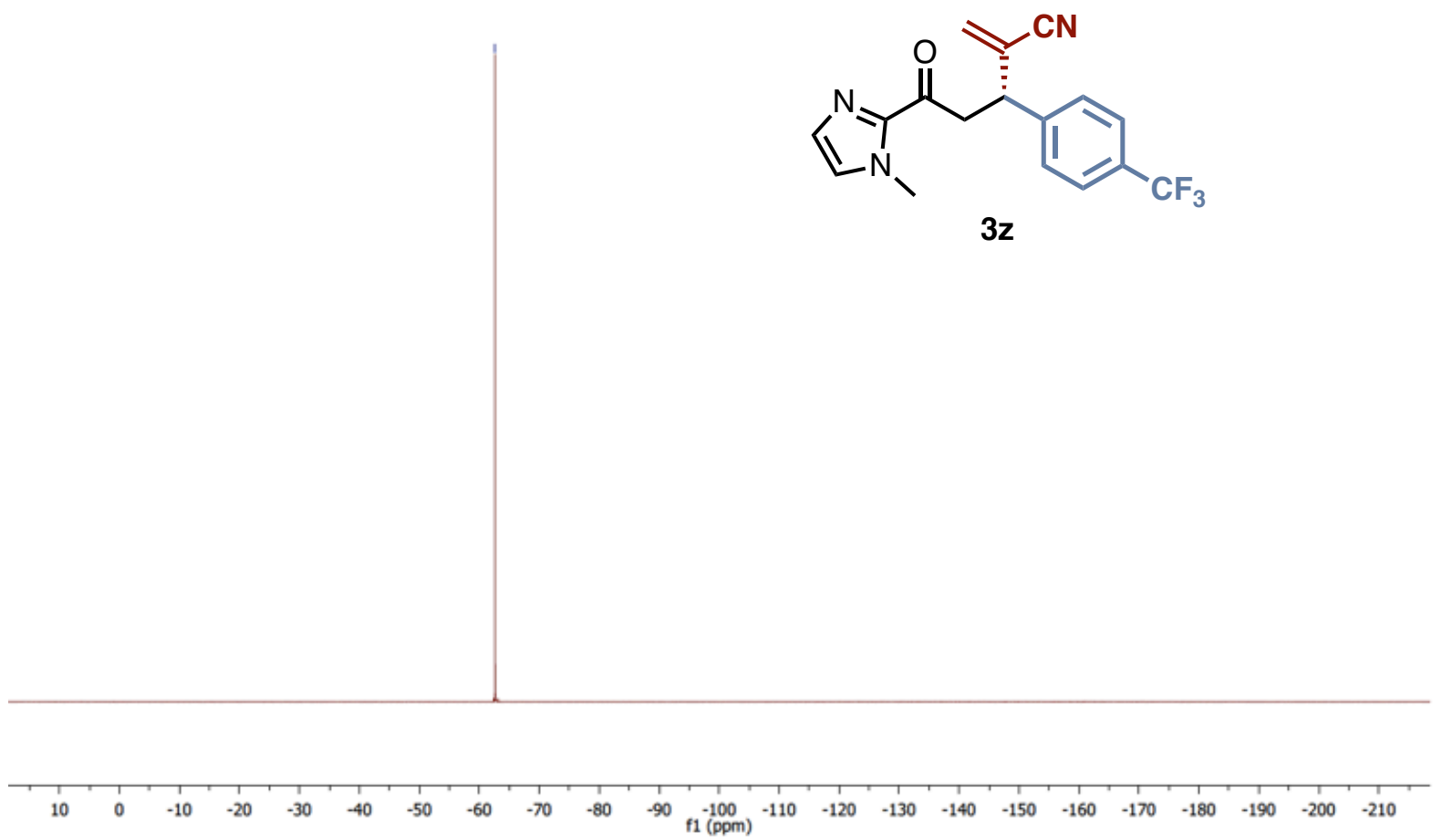

S127 

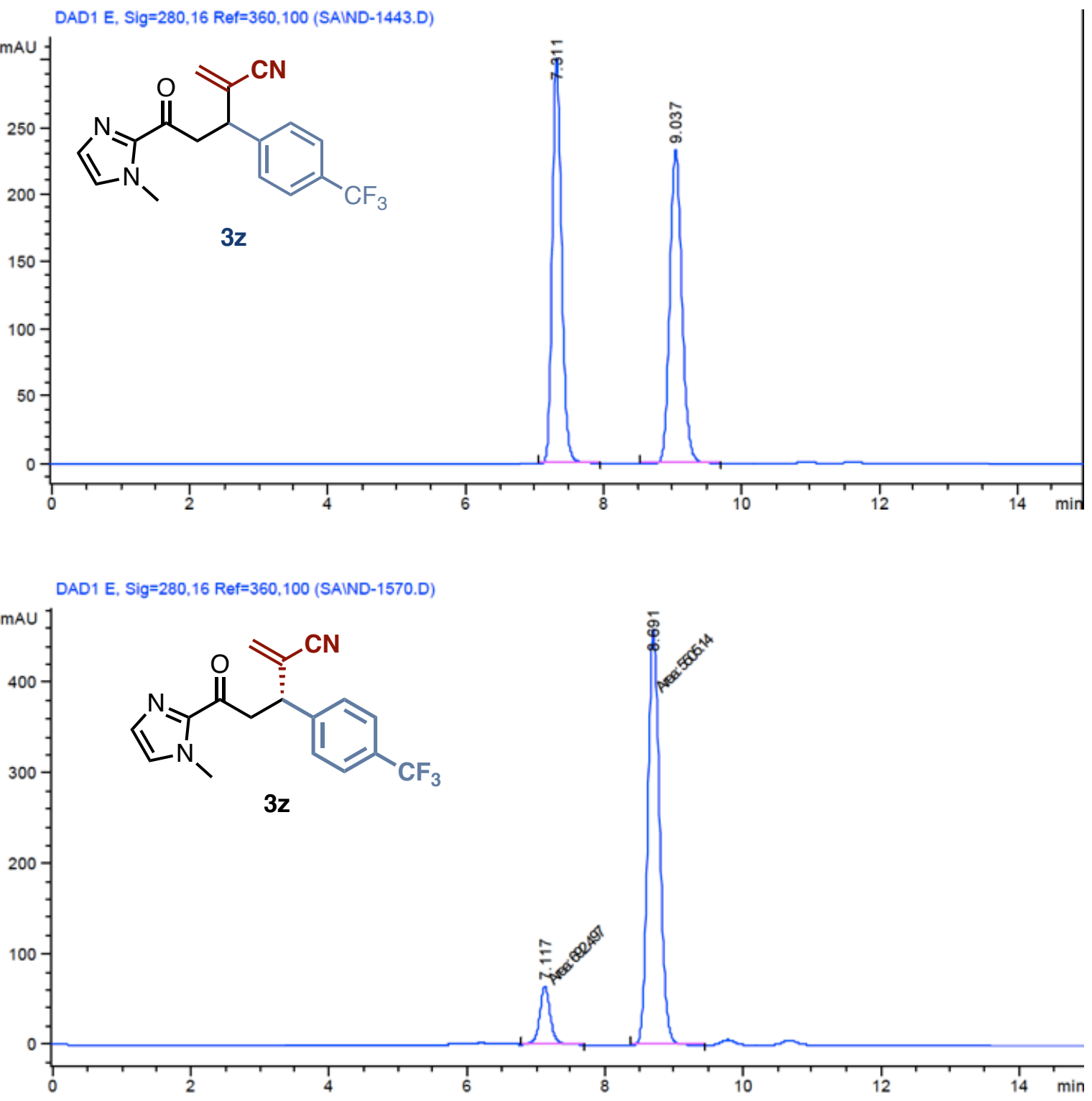

\begin{tabular}{|c|c|c|c|c|c|c|}
\hline $\begin{array}{c}\text { Peak } \\
\dagger\end{array}$ & $\begin{array}{c}\text { RetTime } \\
\text { [min] }\end{array}$ & Type & $\begin{array}{c}\text { Width } \\
\text { [min] }\end{array}$ & $\begin{array}{c}\text { Area } \\
{[\mathrm{mAU} * \mathrm{~S}]}\end{array}$ & $\begin{array}{l}\text { Height } \\
\text { [mAU] }\end{array}$ & $\begin{array}{c}\text { Area } \\
8\end{array}$ \\
\hline & & & & - - - - - & --- & -------1 \\
\hline 1 & 7.117 & MM & 0.1788 & 692.49677 & 64.56555 & 11.1736 \\
\hline 2 & 8.691 & MM & 0.1994 & 5505.13721 & 460.16653 & 88.8264 \\
\hline
\end{tabular}




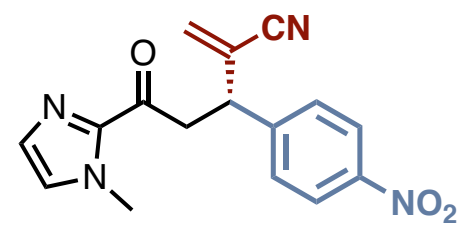

Molecular formula: $\mathrm{C}_{16} \mathrm{H}_{14} \mathrm{~N}_{4} \mathrm{O}_{3}$

$\mathrm{MW}=310.31 \mathrm{~g} \cdot \mathrm{mol}^{-1}$

The title compound was isolated as a yellow oil (43 $\mathrm{mg}, 0.138 \mathrm{mmol}, 51 \%, 76 \%$ ee) using general procedure F. Purified by flash column chromatography over silica gel $($ Hex/EtOAc/Acetone $=7: 2: 1)$.

${ }^{1} \mathrm{H}$ NMR $\left(400 \mathrm{MHz}_{\mathrm{C}} \mathrm{CDCl}_{3}\right) \delta 8.21-8.18(\mathrm{~m}, 2 \mathrm{H}), 7.54-7.50(\mathrm{~m}, 2 \mathrm{H}), 7.15(\mathrm{~d}, J=0.9 \mathrm{~Hz}, 1 \mathrm{H})$, $7.05(\mathrm{~s}, 1 \mathrm{H}), 6.0(\mathrm{~d}, J=0.8 \mathrm{~Hz}, 1 \mathrm{H}), 5.94(\mathrm{~d}, J=1.3 \mathrm{~Hz}, 1 \mathrm{H}), 4.44(\mathrm{t}, J=7.4 \mathrm{~Hz}, 1 \mathrm{H}), 3.94$ $(\mathrm{s}, 3 \mathrm{H}), 3.88-3.75(\mathrm{~m}, 2 \mathrm{H})$.

${ }^{13} \mathrm{C}$ NMR $\left(101 \mathrm{MHz}, \mathrm{CDCl}_{3}\right) \delta 188.1,147.6,146.7,142.5,131.3,129.8,129.1,127.8,125.2$, $124.3,117.4,44.8,41.9,36.2$.

HRMS (ESI) m/z: [M+H] $]^{+}$calcd for $\mathrm{C}_{16} \mathrm{H}_{15} \mathrm{~N}_{4} \mathrm{O}_{3}$ 311.1144; Found: 311.1131.

IR $\left(\mathrm{cm}^{-1}\right):$ 3030, 2955, 2928, 2856, 2225, 1678, 1679, 1523, 1411, 1350.

$[\boldsymbol{a}]_{D}^{20}=-14.3\left(c 1.40, \mathrm{CH}_{2} \mathrm{Cl}_{2}\right)$

HPLC analysis: carried out using a mixture of hexane and isopropyl alcohol (80:20 isocratic), at $1 \mathrm{~mL} \cdot \mathrm{min}^{-1}$, at $30{ }^{\circ} \mathrm{C}$ on an IC column, with UV detection $(\lambda=280 \mathrm{~nm})$. Retention times: 34.59 and $45.24 \mathrm{~min}$. 
${ }^{1} \mathrm{H}-\mathrm{NMR}$ of compound 3 aa $\left(400 \mathrm{MHz}, \mathrm{CDCl}_{3}\right)$

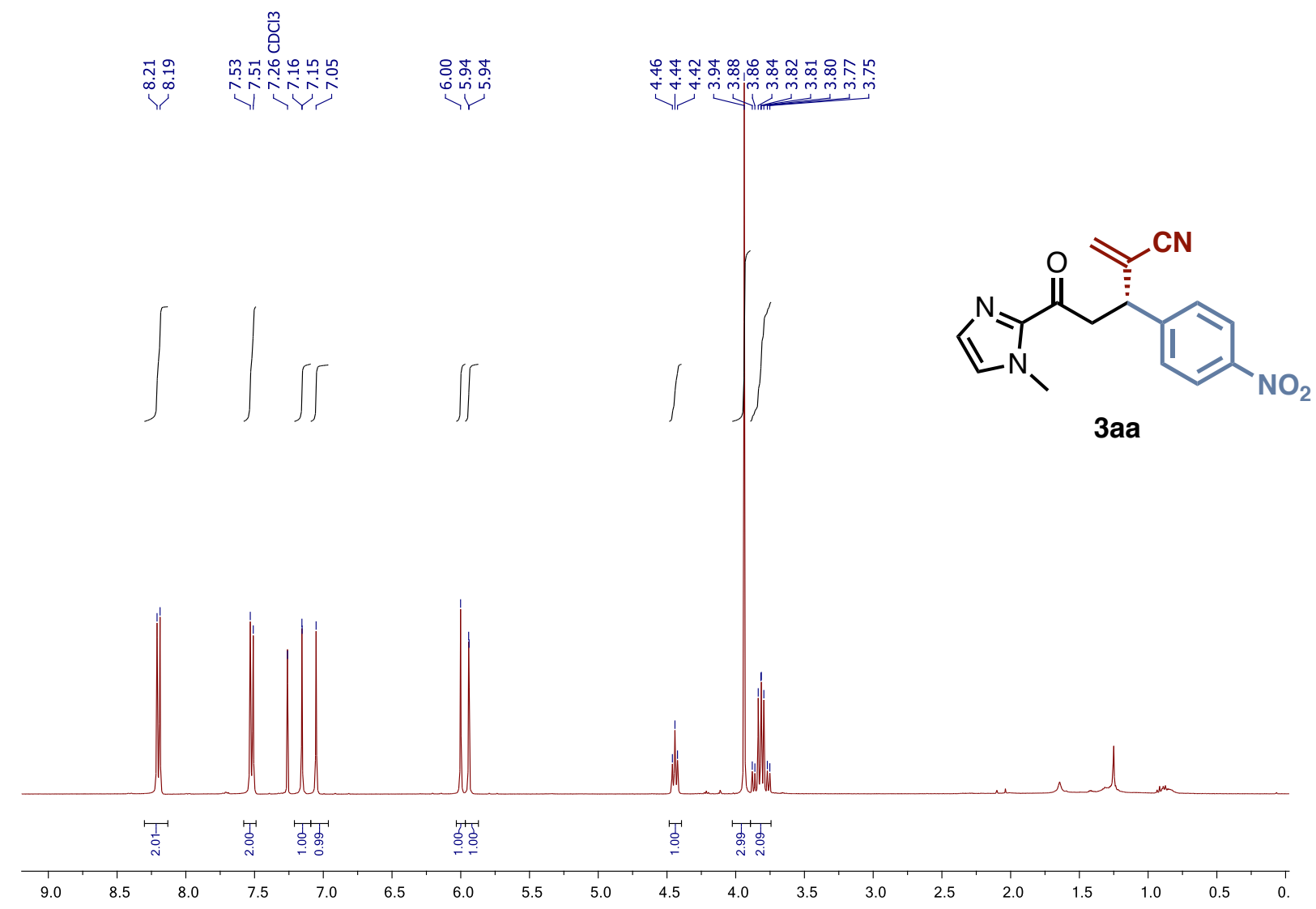

${ }^{13} \mathrm{C}-\mathrm{NMR}$ of compound $3 \mathrm{aa}\left(101 \mathrm{MHz}, \mathrm{CDCl}_{3}\right)$

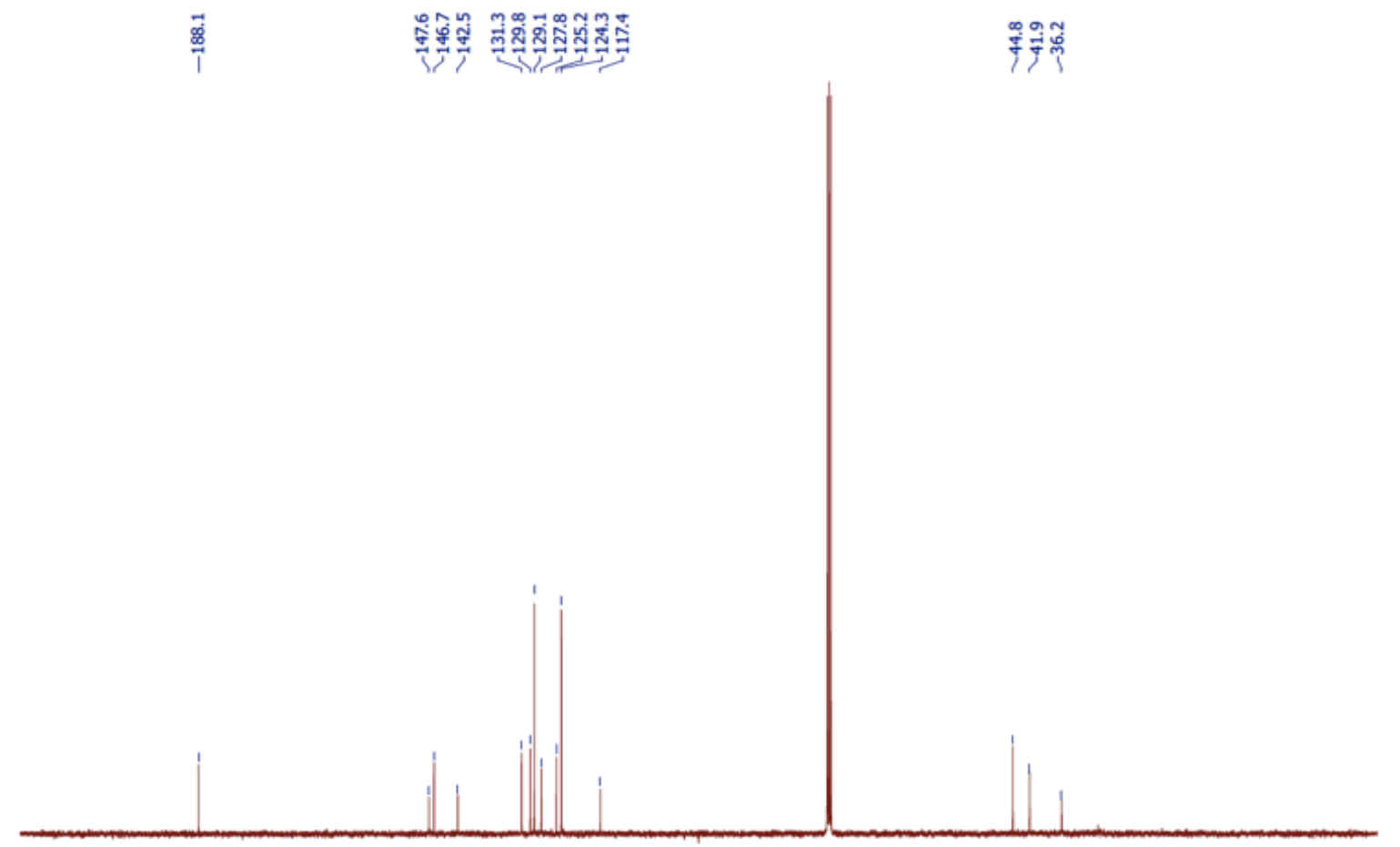

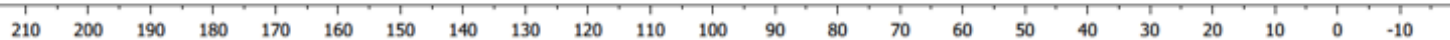



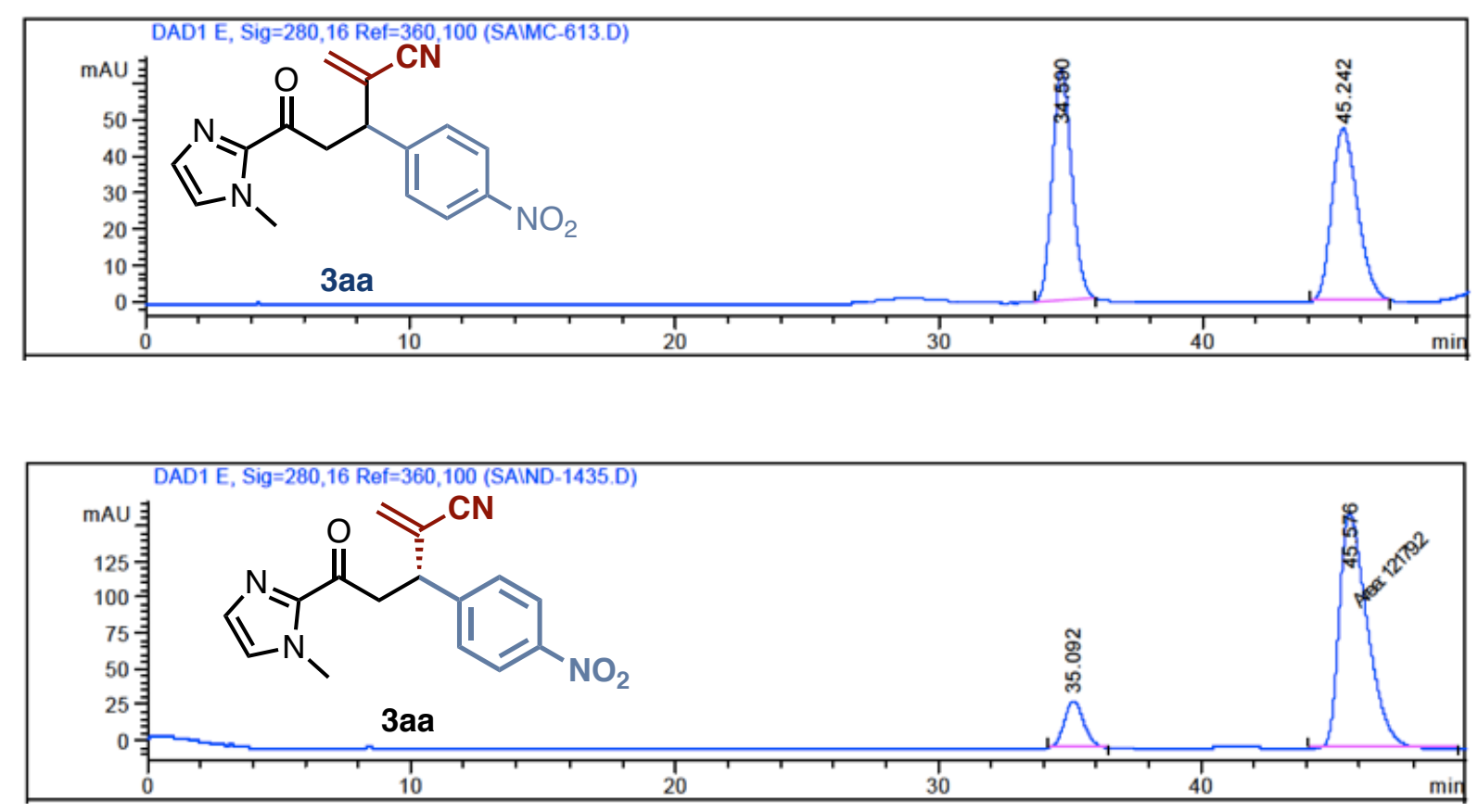

\begin{tabular}{|c|c|c|c|c|c|c|}
\hline $\begin{array}{c}\text { Peak } \\
;\end{array}$ & $\begin{array}{l}\text { RetTime } \\
\text { [min] }\end{array}$ & Type & $\begin{array}{l}\text { Width } \\
\text { [min] }\end{array}$ & $\begin{array}{c}\text { Area } \\
{[\mathrm{mAU} * \mathrm{~s}]}\end{array}$ & $\begin{array}{l}\text { Height } \\
\text { [mAU] }\end{array}$ & $\begin{array}{c}\text { Area } \\
*\end{array}$ \\
\hline 1 & 35.092 & BB & 0.7975 & 1695.62878 & 32.88744 & 12.2209 \\
\hline 2 & 45.576 & $\mathrm{MM}$ & 1.2374 & $1.21792 e^{4}$ & 164.04843 & 87.7791 \\
\hline
\end{tabular}


Methyl 4-(2-cyano-5-(1-methyl-1H-imidazol-2-yl)-5-oxopent-1-en-3-yl)benzoate (3ab)

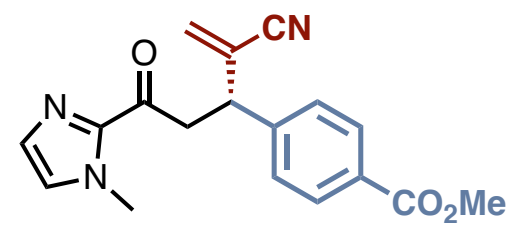

Molecular formula: $\mathrm{C}_{18} \mathrm{H}_{17} \mathrm{~N}_{3} \mathrm{O}_{3}$

$\mathrm{MW}=323.35 \mathrm{~g} \cdot \mathrm{mol}^{-1}$

The title compound was isolated as a clear oil (70 mg, $0.216 \mathrm{mmol}, 80 \%, 81 \%$ ee $)$ using general procedure F. Purified by flash column chromatography over silica gel $($ Hex/EtOAc $=6: 4)$.

${ }^{1} \mathrm{H}$ NMR $\left(400 \mathrm{MHz}, \mathrm{CDCl}_{3}\right) \delta$ 8.01-7.78 (m, 2H), 7.42-7.39 (d, J = 8.0 Hz, 2H), 7.15 (s, 1H), 7.03 (s, 1H), $5.95(\mathrm{~d}, J=0.9 \mathrm{~Hz}, 1 \mathrm{H}), 5.88(\mathrm{~d}, J=1.3 \mathrm{~Hz}, 1 \mathrm{H}), 4.38(\mathrm{t}, J=7.4 \mathrm{~Hz}, 1 \mathrm{H}), 3.93(\mathrm{~s}, 3 \mathrm{H})$, $3.89(\mathrm{~s}, 3 \mathrm{H}), 3.80(\mathrm{~d}, J=7.5 \mathrm{~Hz}, 2 \mathrm{H})$.

${ }^{13} \mathrm{C}$ NMR $\left(101 \mathrm{MHz}, \mathrm{CDCl}_{3}\right) \delta 188.5,166.7,144.3,142.5,130.7,130.2,129.5,128.0,127.5$, $117.6,52.1,44.8,41.9,36.1,31.6,22.6,14.1$.

HRMS (ESI) m/z: [M+H] $]^{+}$calcd for $\mathrm{C}_{18} \mathrm{H}_{18} \mathrm{~N}_{3} \mathrm{O}_{3}$ 324.1348; found: 324.1345 .

IR $\left(\mathrm{cm}^{-1}\right):$ 2954, 2928, 2225, 1721, 1678, 1613, 1411, 1283, 1112.

$[\boldsymbol{a}]_{\boldsymbol{D}}^{\mathbf{2 0}}=-8.0\left(\mathrm{c} 1.70, \mathrm{CH}_{2} \mathrm{Cl}_{2}\right)$

HPLC analysis: carried out using a mixture of hexane and isopropyl alcohol (80:20 isocratic), at $1 \mathrm{~mL} \cdot \mathrm{min}^{-1}$, at $30{ }^{\circ} \mathrm{C}$ on an IA column, with UV detection $(\lambda=280 \mathrm{~nm})$. Retention times: 34.59 and $45.24 \mathrm{~min}$. 


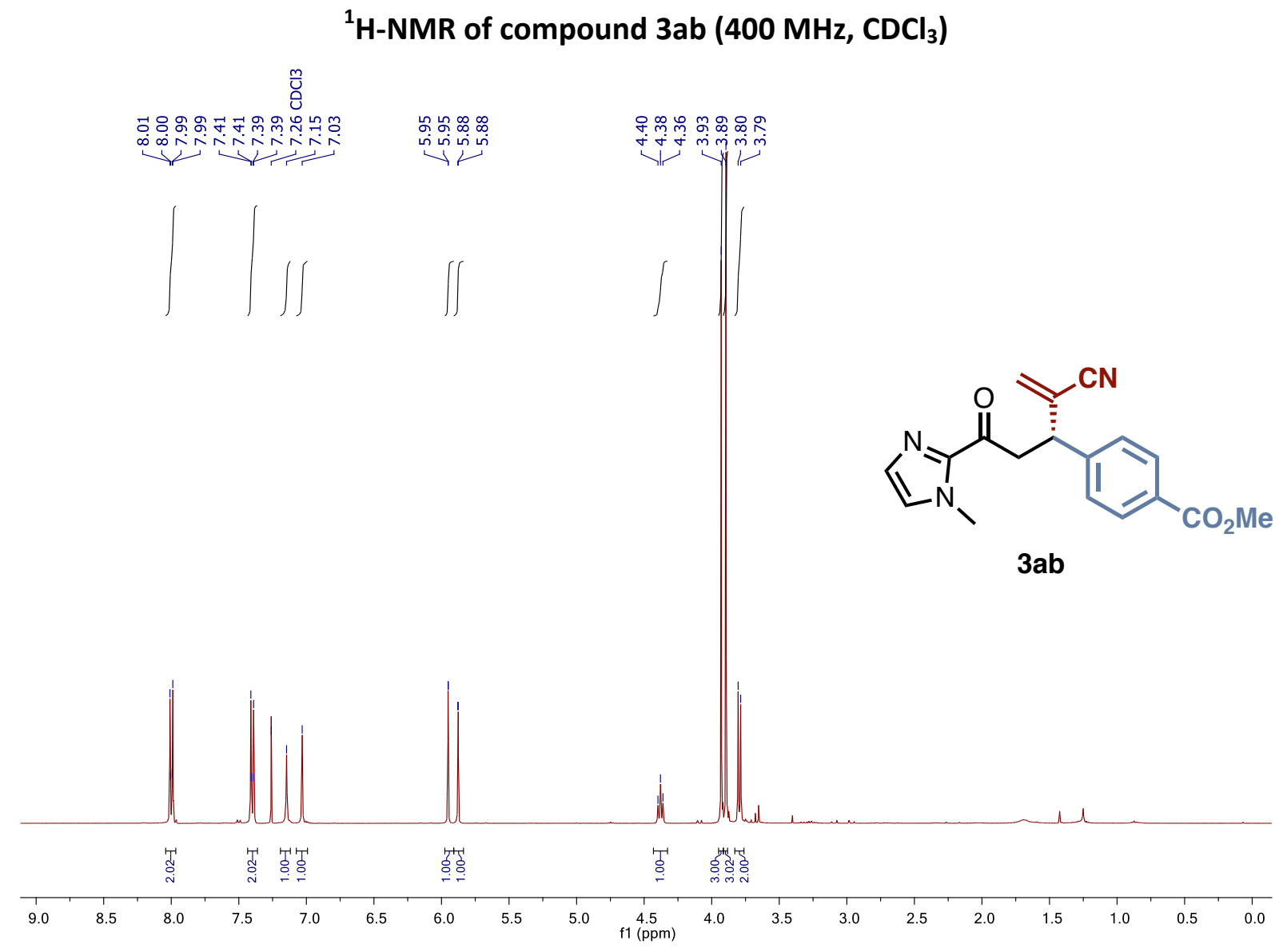

${ }^{13} \mathrm{C}-\mathrm{NMR}$ of compound $3 \mathrm{ab}\left(101 \mathrm{MHz}, \mathrm{CDCl}_{3}\right.$ )

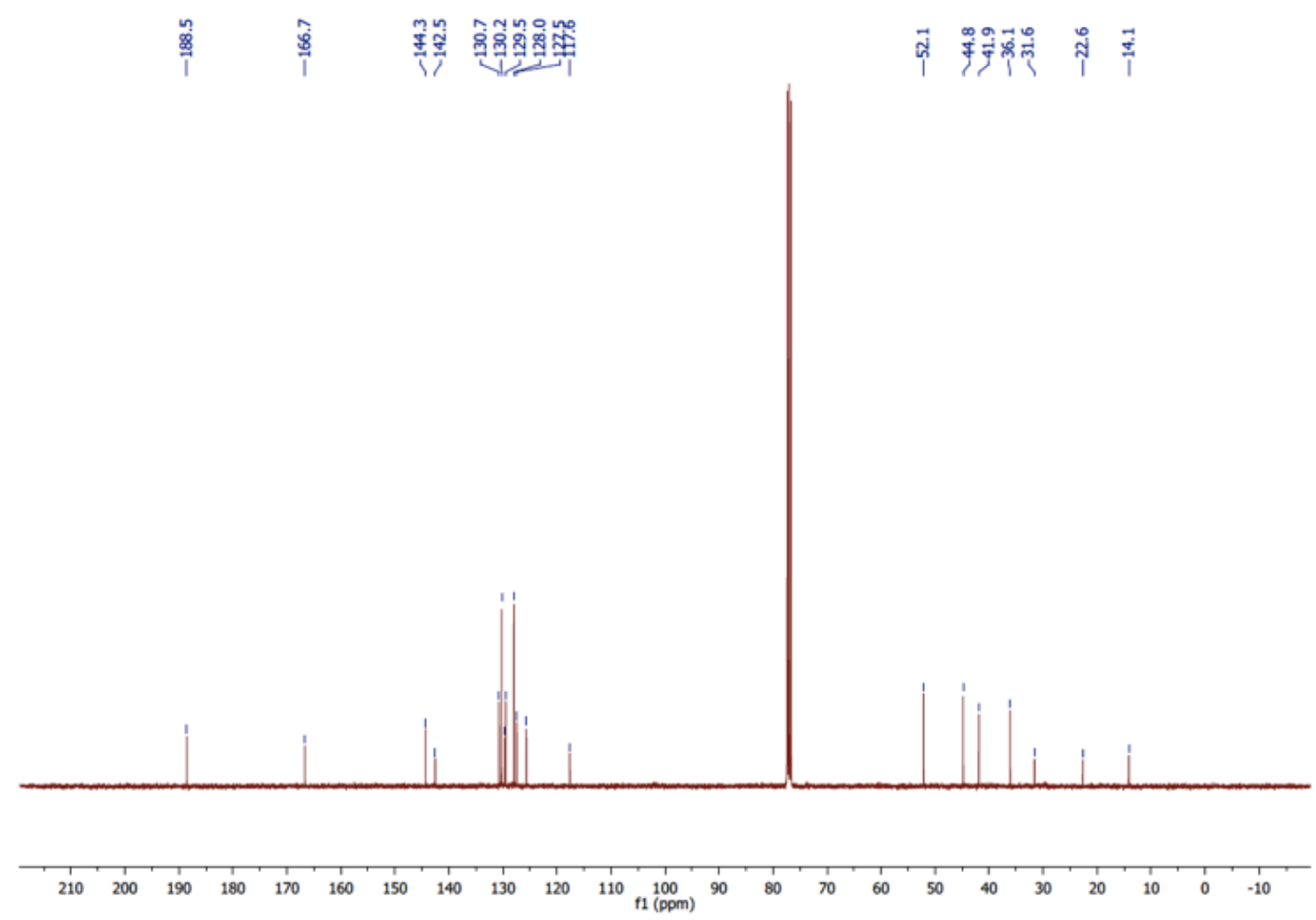



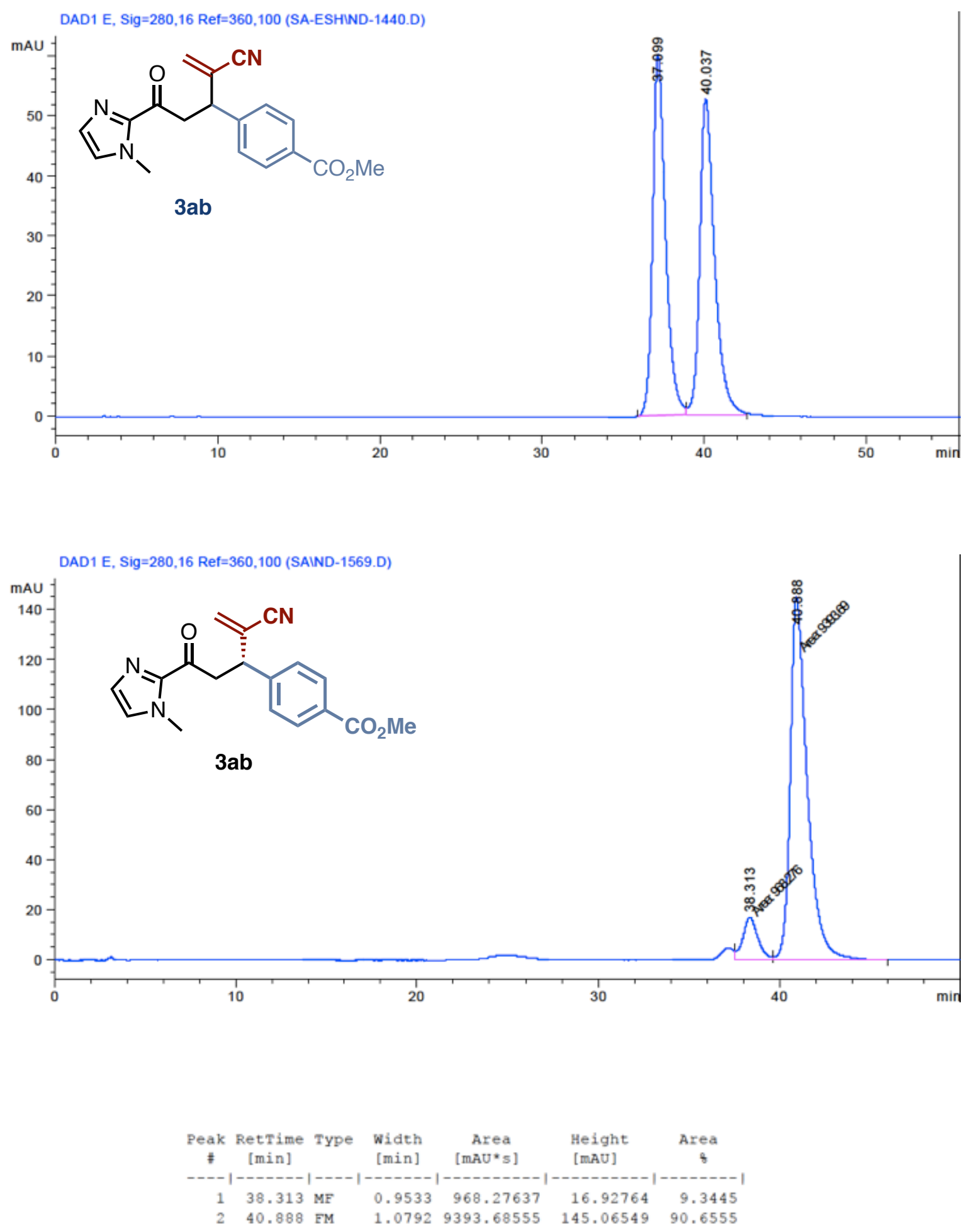


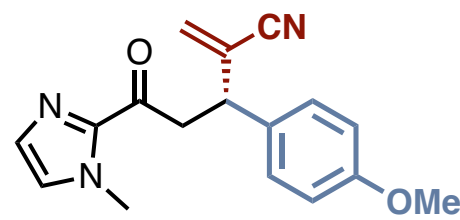

Molecular formula: $\mathrm{C}_{17} \mathrm{H}_{17} \mathrm{~N}_{3} \mathrm{O}_{2}$

$\mathrm{MW}=295.34 \mathrm{~g} \cdot \mathrm{mol}^{-1}$

The title compound was isolated as an amorphous solid (47 mg, $0.162 \mathrm{mmol}, 60 \%, 73 \%$ ee $)$ using general procedure F. Purified by flash column chromatography over silica gel $($ Hex/EtOAc/Acetone $=7: 2: 1)$.

${ }^{1}$ H NMR (400 MHz, $\left.\mathrm{CDCl}_{3}\right) \delta$ 7.26-7.20 (m, 2H), $7.14(\mathrm{~s}, 1 \mathrm{H}), 7.02(\mathrm{~s}, 1 \mathrm{H}), 6.88-6.82(\mathrm{~m}, 2 \mathrm{H})$, $5.88(\mathrm{~s}, 1 \mathrm{H}), 5.82(\mathrm{~s}, 1 \mathrm{H}), 4.27(\mathrm{t}, J=7.1 \mathrm{~Hz}, 1 \mathrm{H}), 3.93(\mathrm{~s}, 3 \mathrm{H}), 3.78(\mathrm{~s}, 3 \mathrm{H}), 3.85-3.67(\mathrm{~m}, 2 \mathrm{H})$.

${ }^{13} \mathrm{C}$ NMR $\left(101 \mathrm{MHz}, \mathrm{CDCl}_{3}\right) \delta 189.3,159.1,142.9,131.3,129.8,129.5,129.1(2 \mathrm{C}), 127.4$, $126.9,118.2,114.4(2 \mathrm{C}), 55.4,44.3,42.3,36.2$.

HRMS (ESI) m/z: [M+H] $]^{+}$calcd for $\mathrm{C}_{17} \mathrm{H}_{18} \mathrm{~N}_{3} \mathrm{O}_{2}$ 296.1399; found: 296.1369 .

IR $\left(\mathrm{cm}^{-1}\right):$ 2959, 2928, 2855, 2224, 1676, 1613, 1514, 1467, 1410, 1253, 1182, 1035.

$[\boldsymbol{a}]_{\boldsymbol{D}}^{\mathbf{2 0}}=-9.7\left(\mathrm{c} 1.73, \mathrm{CH}_{2} \mathrm{Cl}_{2}\right)$

HPLC analysis: carried out using a mixture of hexane and isopropyl alcohol (70:30 isocratic), at $1 \mathrm{~mL} \cdot \mathrm{min}^{-1}$, at $25{ }^{\circ} \mathrm{C}$ on an IC column, with UV detection $(\lambda=280 \mathrm{~nm})$. Retention times: 12.7 and $14.9 \mathrm{~min}$. 


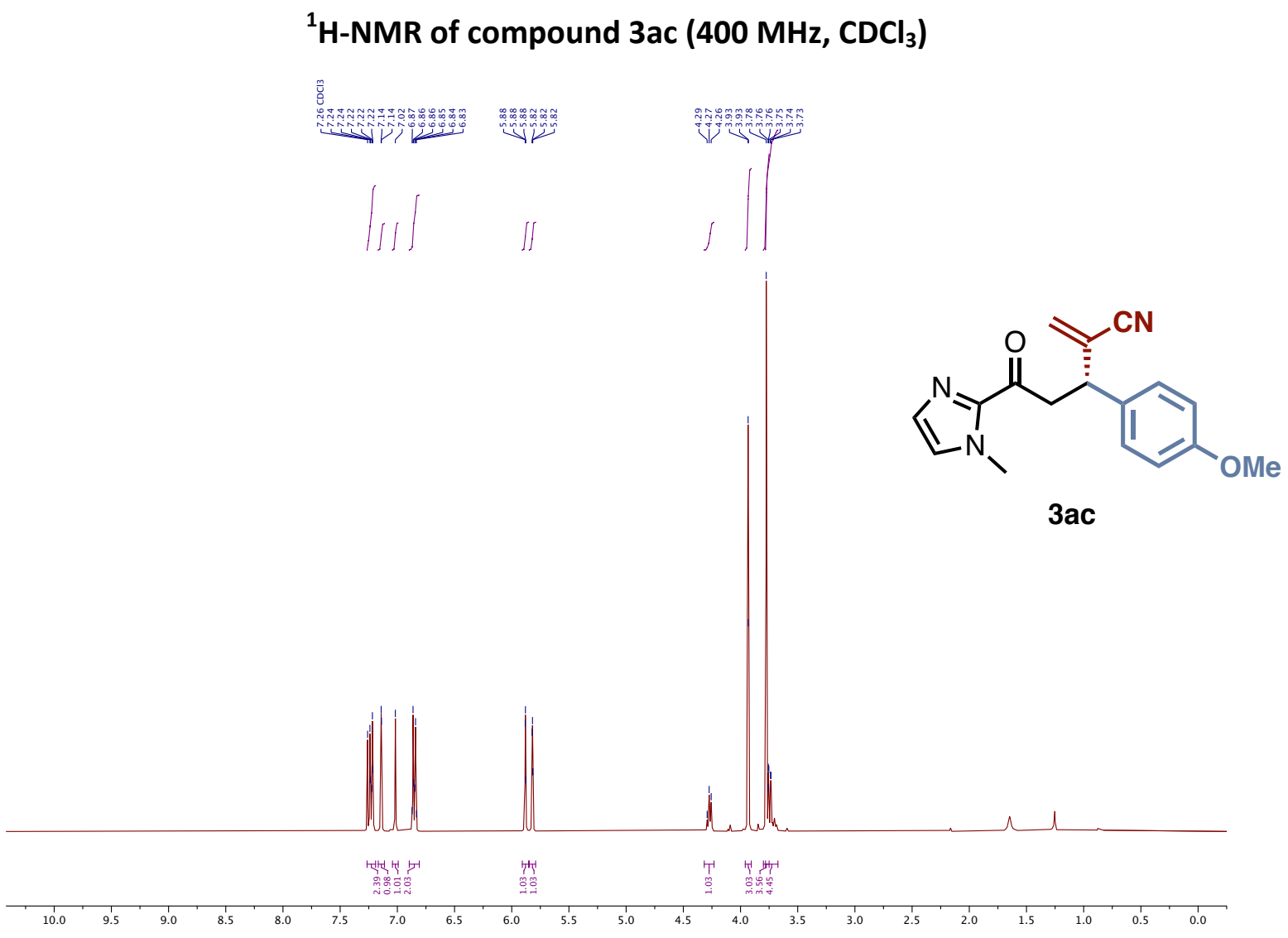

${ }^{13} \mathrm{C}-\mathrm{NMR}$ of compound $3 \mathrm{ac}\left(101 \mathrm{MHz}, \mathrm{CDCl}_{3}\right)$
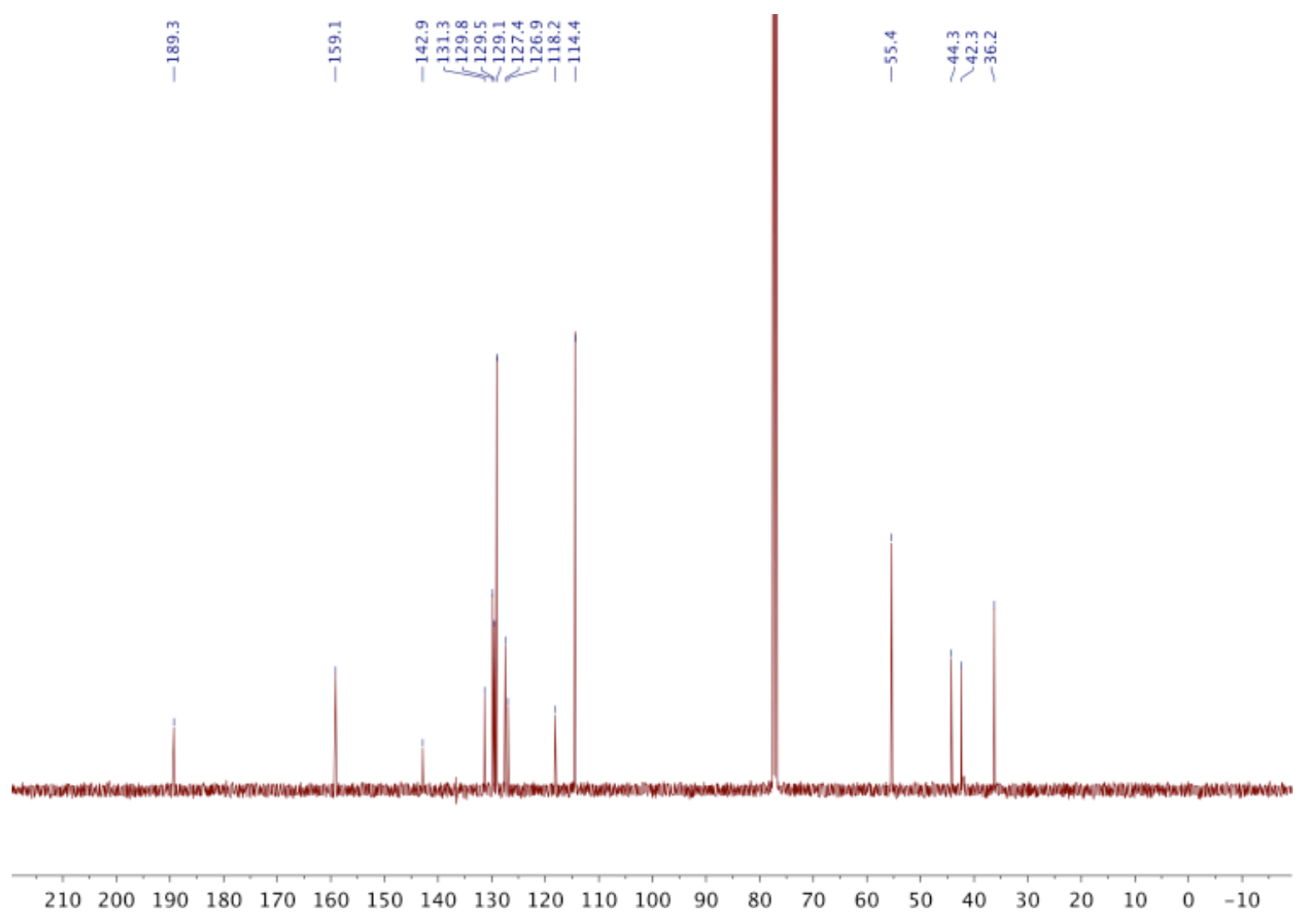

S136 

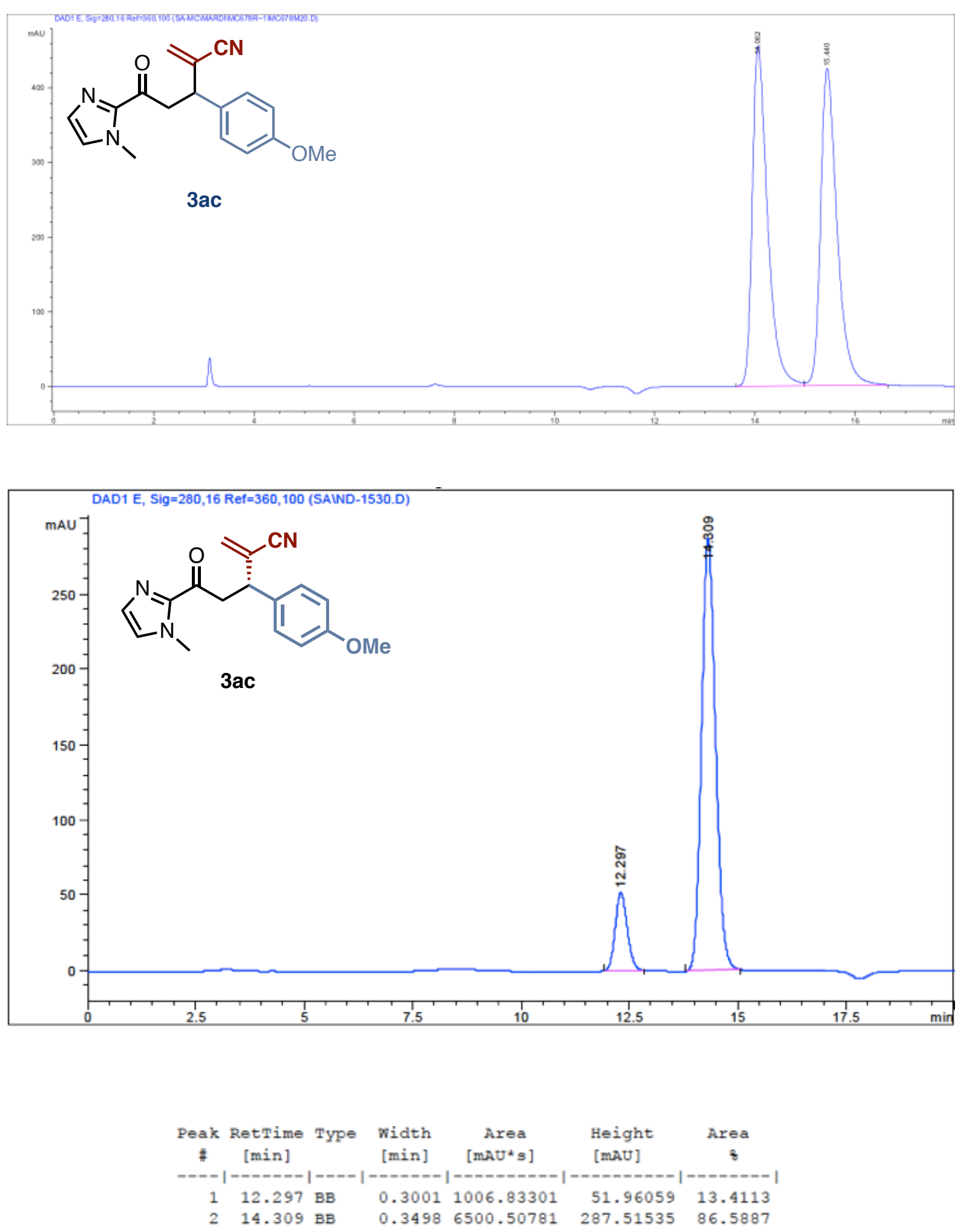
3-(Furan-2-yl)-5-(1-methyl-1H-imidazol-2-yl)-2-methylene-5-oxopentanenitrile (3ad)

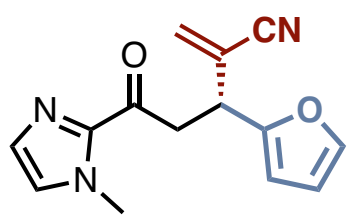

Molecular formula: $\mathrm{C}_{14} \mathrm{H}_{13} \mathrm{~N}_{3} \mathrm{O}_{2}$

$\mathbf{M W}=255.28 \mathrm{~g} \cdot \mathrm{mol}^{-1}$

The title compound was isolated as a beige solid (35 mg, $0.132 \mathrm{mmol}, 49 \%, 43 \%$ ee ) using general procedure F. Purified by flash column chromatography over silica gel (Toluene/Acetone= 9:1).

${ }^{1}$ H NMR $\left(400 \mathrm{MHz}^{\mathrm{C}} \mathrm{CDCl}_{3}\right) \delta 7.35(\mathrm{t}, J=1.9,0.8 \mathrm{~Hz}, 1 \mathrm{H}), 7.16(\mathrm{~d}, J=0.9 \mathrm{~Hz}, 1 \mathrm{H}), 7.05$ $(\mathrm{d}, J=0.9 \mathrm{~Hz}, 1 \mathrm{H}), 6.31$ (dd, $J=3.3,1.9 \mathrm{~Hz}, 1 \mathrm{H}), 6.26(\mathrm{dt}, J=3.3,0.8 \mathrm{~Hz}, 1 \mathrm{H}), 5.97(\mathrm{~s}, 1 \mathrm{H})$, $5.89(\mathrm{~d}, J=1.1 \mathrm{~Hz}, 1 \mathrm{H}), 4.42(\mathrm{dd}, J=7.8,7.0 \mathrm{~Hz}, 1 \mathrm{H}), 3.98(\mathrm{~s}, 3 \mathrm{H}), 3.83(\mathrm{dd}, J=17.5,7.0 \mathrm{~Hz}$, $1 \mathrm{H}), 3.70(\mathrm{dd}, J=17.5,7.8 \mathrm{~Hz}, 1 \mathrm{H})$.

${ }^{13} \mathrm{C}$ NMR $\left(101 \mathrm{MHz} \mathrm{CDCl}_{3}\right) \delta 188.6,152.2,142.7,142.5,131.7,129.6,127.5,124.0,117.5$, $110.6,107.3,40.7,39.0,36.2$.

HRMS (ESI) m/z: [M+H] $]^{+}$calcd for $\mathrm{C}_{14} \mathrm{H}_{14} \mathrm{~N}_{3} \mathrm{O}_{2}$ 256.1086; found: 256.1086 .

IR $\left(\mathrm{cm}^{-1}\right): 2959,2926,2225,1676,1410,1158$.

$[a]_{D}^{20}=+18.5\left(c 0.55, \mathrm{CH}_{2} \mathrm{Cl}_{2}\right)$

HPLC analysis: carried out using a mixture of hexane and isopropyl alcohol (70:30 isocratic) at $1 \mathrm{ml} \cdot \mathrm{min}^{-1}$, at $25{ }^{\circ} \mathrm{C}$ on an IC column, with UV detection $(\lambda=280 \mathrm{~nm})$. Retention times: 10.9 and $12.5 \mathrm{~min}$. 


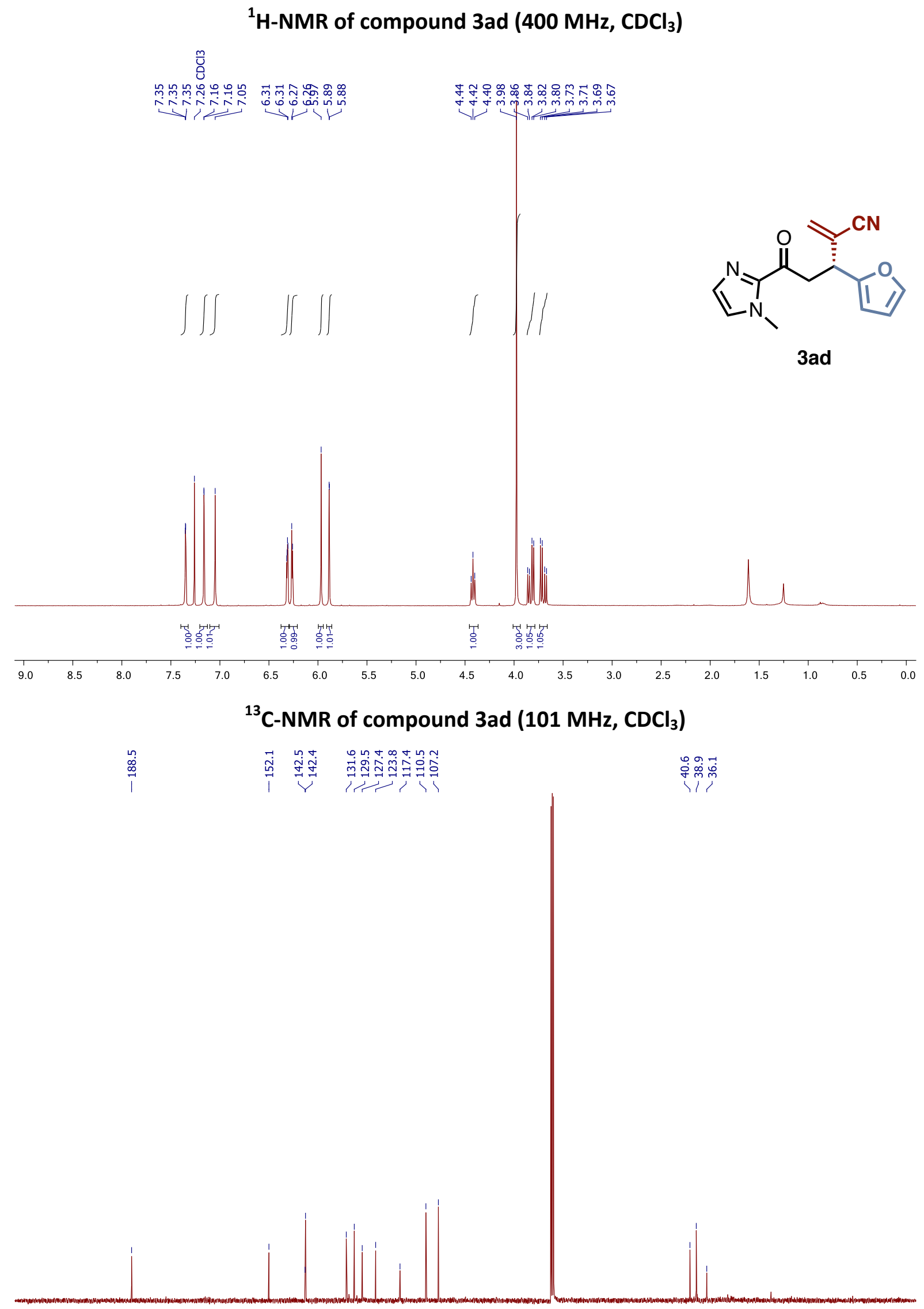



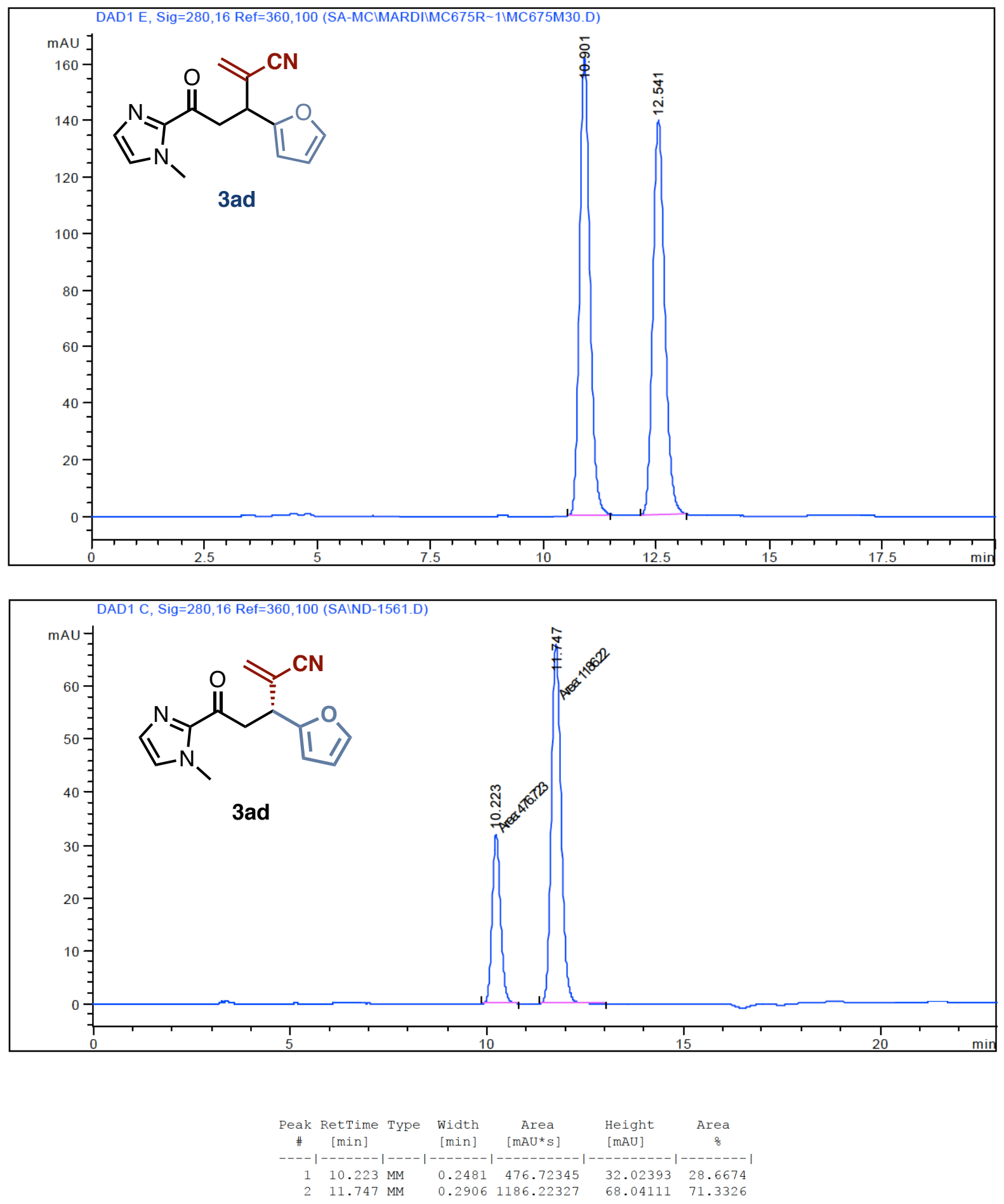


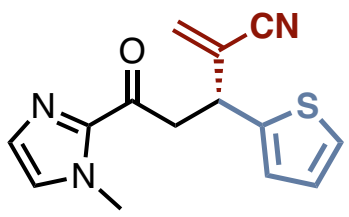

Molecular formula: $\mathrm{C}_{14} \mathrm{H}_{13} \mathrm{~N}_{3} \mathrm{OS}$

$\mathbf{M W}=271.34 \mathrm{~g} \cdot \mathrm{mol}^{-1}$

The title compound was isolated as a pale yellow oil $(50 \mathrm{mg}, 0.184 \mathrm{mmol}, 68 \%, 65 \%$ ee) using general procedure F. Purified by flash column chromatography over silica gel (Hex/EtOAc/Acetone= 7:2:1).

${ }^{1} \mathrm{H}$ NMR $\left(400 \mathrm{MHz}, \mathrm{CDCl}_{3}\right) \delta 7.21(\mathrm{dd}, J=5.1,1.2 \mathrm{~Hz}, 1 \mathrm{H}), 7.17(\mathrm{~d}, J=0.9 \mathrm{~Hz}, 1 \mathrm{H}), 7.04$ (d, J=0.9 Hz, 1H), $7.02(\mathrm{dt}, J=3.6,1.2 \mathrm{~Hz}, 1 \mathrm{H}), 6.96$ (dd, $J=5.1,3.6 \mathrm{~Hz}, 1 \mathrm{H}), 5.94$ (d, J = 0.7 $\mathrm{Hz}, 1 \mathrm{H}), 5.90(\mathrm{~d}, J=1.2 \mathrm{~Hz}, 1 \mathrm{H}), 4.61(\mathrm{dd}, J=7.6,7.1 \mathrm{~Hz}, 1 \mathrm{H}), 3.97(\mathrm{~s}, 3 \mathrm{H}), 3.86(\mathrm{dd}, J=17.4$, $7.2 \mathrm{~Hz}, 1 \mathrm{H}), 3.80(\mathrm{dd}, J=17.4,7.6 \mathrm{~Hz}, 1 \mathrm{H})$.

${ }^{13} \mathrm{C}$ NMR $\left(101 \mathrm{MHz}, \mathrm{CDCl}_{3}\right) \delta 188.6,142.7,142.4,130.8,129.7,127.6,127.3,126.2,125.6$, $125.1,117.6,43.3,40.4,36.3$.

HRMS (ESI) m/z: [M+H] ${ }^{+}$calcd for $\mathrm{C}_{14} \mathrm{H}_{14} \mathrm{~N}_{3} \mathrm{OS} 272.0857$; found: 272.0857 .

IR $\left(\mathrm{cm}^{-1}\right): 3108,2957,2924,222^{\prime}, 1737,1674,1406,1292,1158$.

$[a]_{D}^{20}=-11.7\left(c\right.$ 1.57, $\left.\mathrm{CH}_{2} \mathrm{Cl}_{2}\right)$

HPLC analysis: carried out using a mixture of hexane and isopropyl alcohol (70:30 isocratic) at $1 \mathrm{ml} \cdot \mathrm{min}^{-1}$, at $25^{\circ} \mathrm{C}$ on an IC column, with UV detection $(\lambda=280 \mathrm{~nm})$. Retention times: 10.66 and $12.71 \mathrm{~min}$. 
${ }^{1} \mathrm{H}-\mathrm{NMR}$ of compound 3ae (400 MHz, $\left.\mathrm{CDCl}_{3}\right)$

品

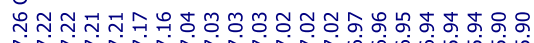

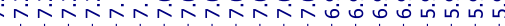

ஜூ்ְ

f

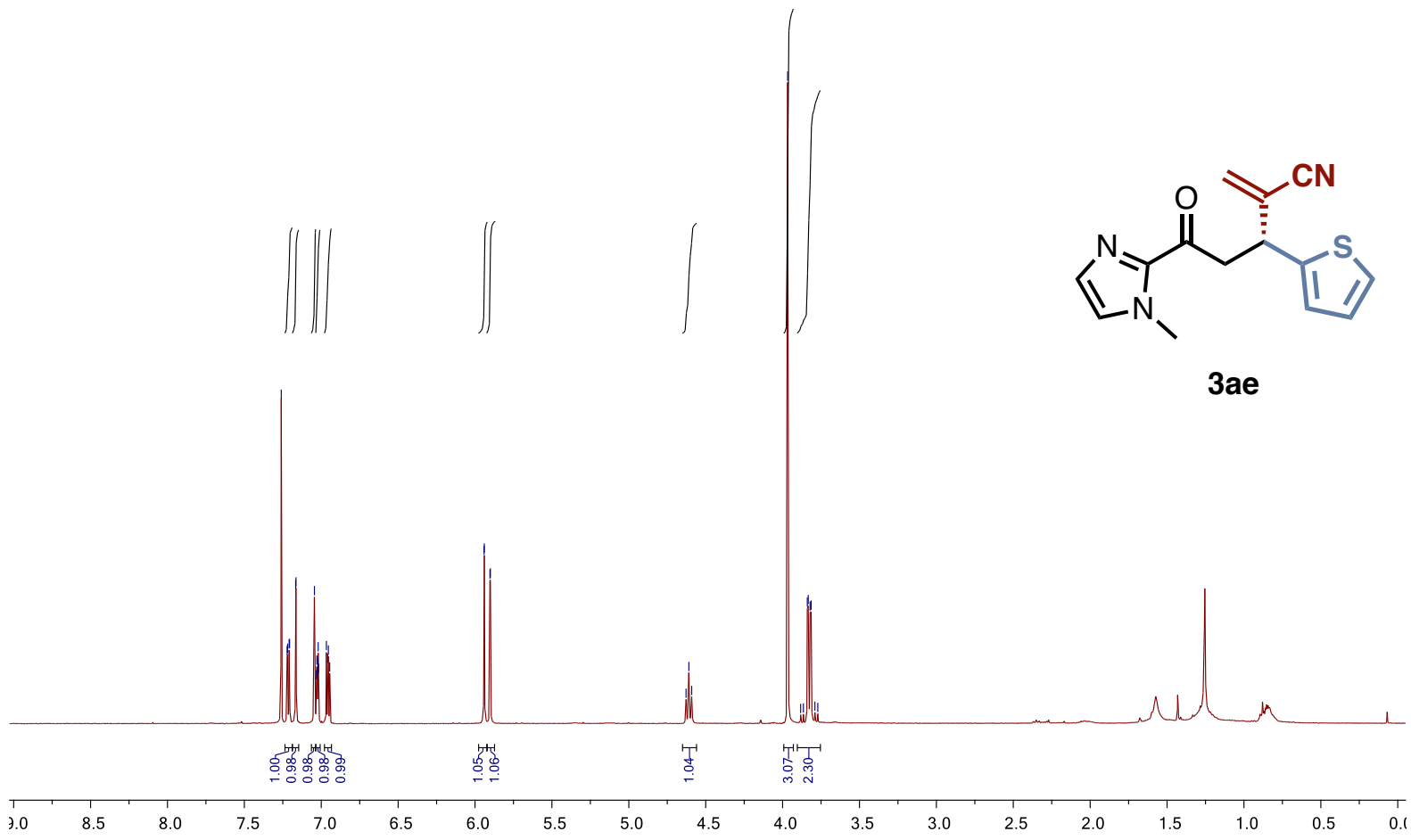

${ }^{13} \mathrm{C}-\mathrm{NMR}$ of compound 3ae (101 $\mathrm{MHz}, \mathrm{CDCl}_{3}$ )

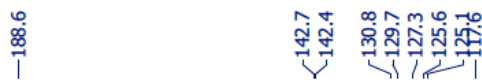

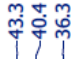

$\begin{array}{lllllllllllllllllllllll}210 & 200 & 190 & 180 & 170 & 160 & 150 & 140 & 130 & 120 & 110 & 100 & 90 & 80 & 70 & 60 & 50 & 40 & 30 & 20 & 10 & 0 & -10\end{array}$ 

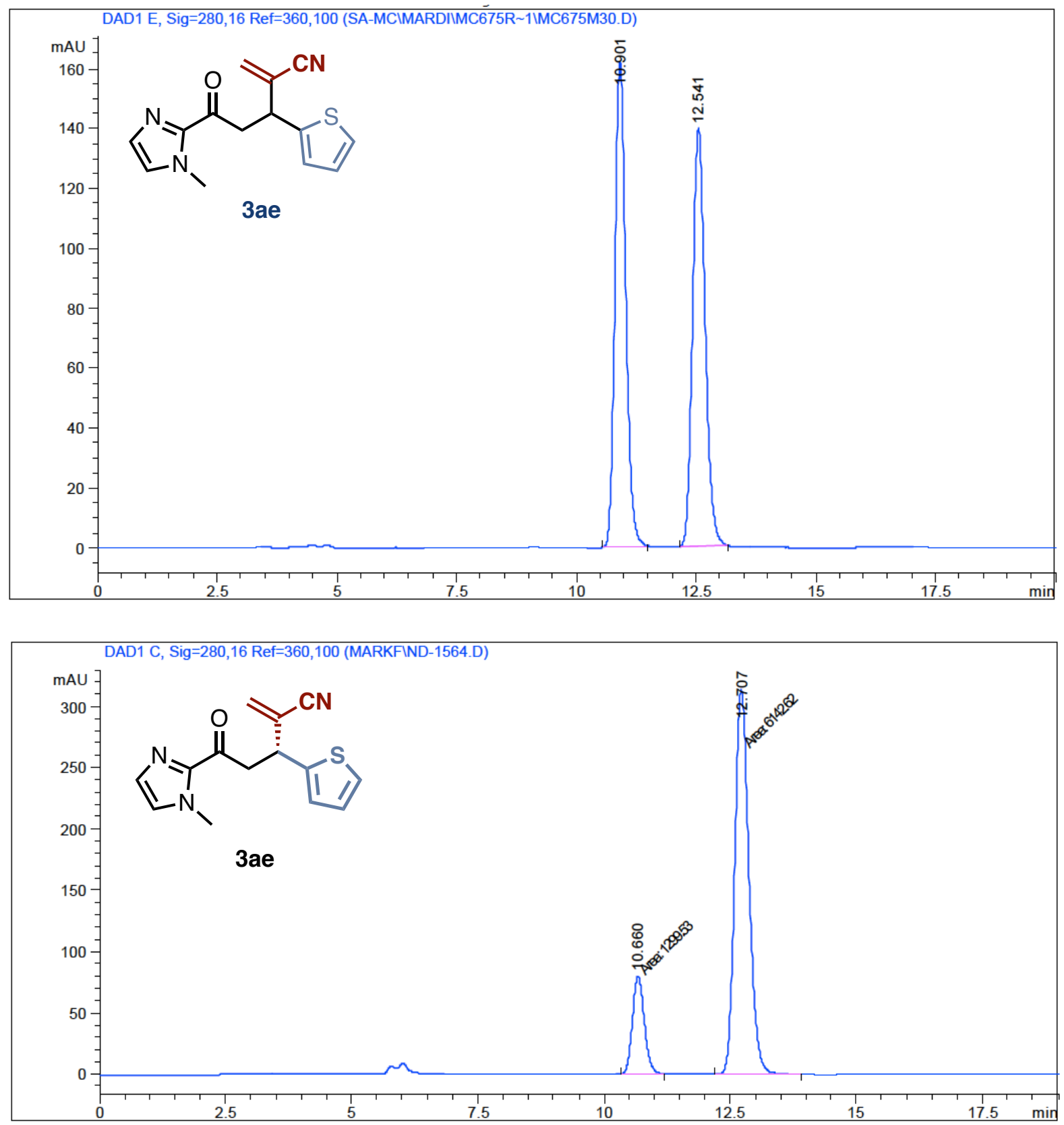

\begin{tabular}{|c|c|c|c|c|c|c|}
\hline $\begin{array}{c}\text { Peak } \\
\#\end{array}$ & $\begin{array}{c}\text { RetTime } \\
\text { [min] }\end{array}$ & Type & $\begin{array}{c}\text { Width } \\
\text { [min] }\end{array}$ & $\begin{array}{c}\text { Area } \\
{\left[\mathrm{mAU}{ }^{\star} \mathrm{S}\right]}\end{array}$ & $\begin{array}{l}\text { Height } \\
\text { [mAU] }\end{array}$ & $\begin{array}{c}\text { Area } \\
\frac{\circ}{8}\end{array}$ \\
\hline & & & & tes & ---- & ---1 \\
\hline 1 & 10.660 & MM & 0.270 & 1299.52673 & 80.04704 & 17. \\
\hline 2 & 12.707 & MM & 0.3259 & 6142.62305 & 314.09473 & 82.5383 \\
\hline
\end{tabular}


(E)-3-[2-(1-Methyl-1H-imidazol-2-yl)-2-oxoethyl]-2-methylenehex-4-enenitrile (3af)

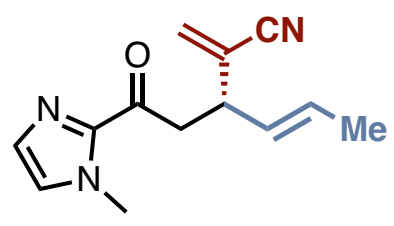

Molecular formula: $\mathrm{C}_{13} \mathrm{H}_{15} \mathrm{~N}_{3} \mathrm{O}$

$\mathrm{MW}=229.28 \mathrm{~g} \cdot \mathrm{mol}^{-1}$

The title compound was isolated as an amorphous solid (31 mg, $0.135 \mathrm{mmol}, 50 \%, 84 \%$ ee) using general procedure $\mathbf{F}$ (only 1.0 equiv. of TBAH was used to prevent epimerization). Purified by flash column chromatography over silica gel (Hex/EtOAc/Acetone= 8:1:1).

${ }^{1} \mathrm{H}$ NMR $\left(400 \mathrm{MHz}, \mathrm{CDCl}_{3}\right) \delta 7.14(\mathrm{~s}, 1 \mathrm{H}), 7.03(\mathrm{~s}, 1 \mathrm{H}), 5.87(\mathrm{~s}, 1 \mathrm{H}), 5.81(\mathrm{~s}, 1 \mathrm{H}), 5.64(\mathrm{~m}, 1 \mathrm{H})$, $5.42(\mathrm{ddd}, J=15.4,7.5,1.9 \mathrm{~Hz}, 1 \mathrm{H}), 3.98(\mathrm{~s}, 3 \mathrm{H}), 3.65(\mathrm{q}, J=7.5 \mathrm{~Hz}, 1 \mathrm{H}), 3.51-3.36$ (m, 2H), $1.68(d, J=6.3 \mathrm{~Hz}, 3 \mathrm{H})$.

${ }^{13} \mathrm{C}$ NMR $\left(101 \mathrm{MHz}, \mathrm{CDCl}_{3}\right) \delta 189.7,142.9,130.0,130.0,129.4,129.0,129.0,127.4,126.1$, $118.1,42.6,42.1,36.3,18.1$.

HRMS (ESI) m/z: [M+H] $]^{+}$calcd for $\mathrm{C}_{13} \mathrm{H}_{16} \mathrm{~N}_{3} \mathrm{O}$ 230.1293; found: 230.1270 .

IR $\left(\mathrm{cm}^{-1}\right):$ 2963, 2922, 2224, 1676, 1467, 1408, 1292, 1158.

$[\boldsymbol{a}]_{\boldsymbol{D}}^{\mathbf{2 0}}=+38.8\left(c 0.63, \mathrm{CH}_{2} \mathrm{Cl}_{2}\right)$

HPLC analysis: carried out using a mixture of hexane and isopropyl alcohol (80:20 isocratic) at $1 \mathrm{~mL} \cdot \mathrm{min}^{-1}$, at $25^{\circ} \mathrm{C}$ on an IC column, with UV detection $(\lambda=280 \mathrm{~nm})$. Retention times: 12.40 and $15.00 \mathrm{~min}$. 


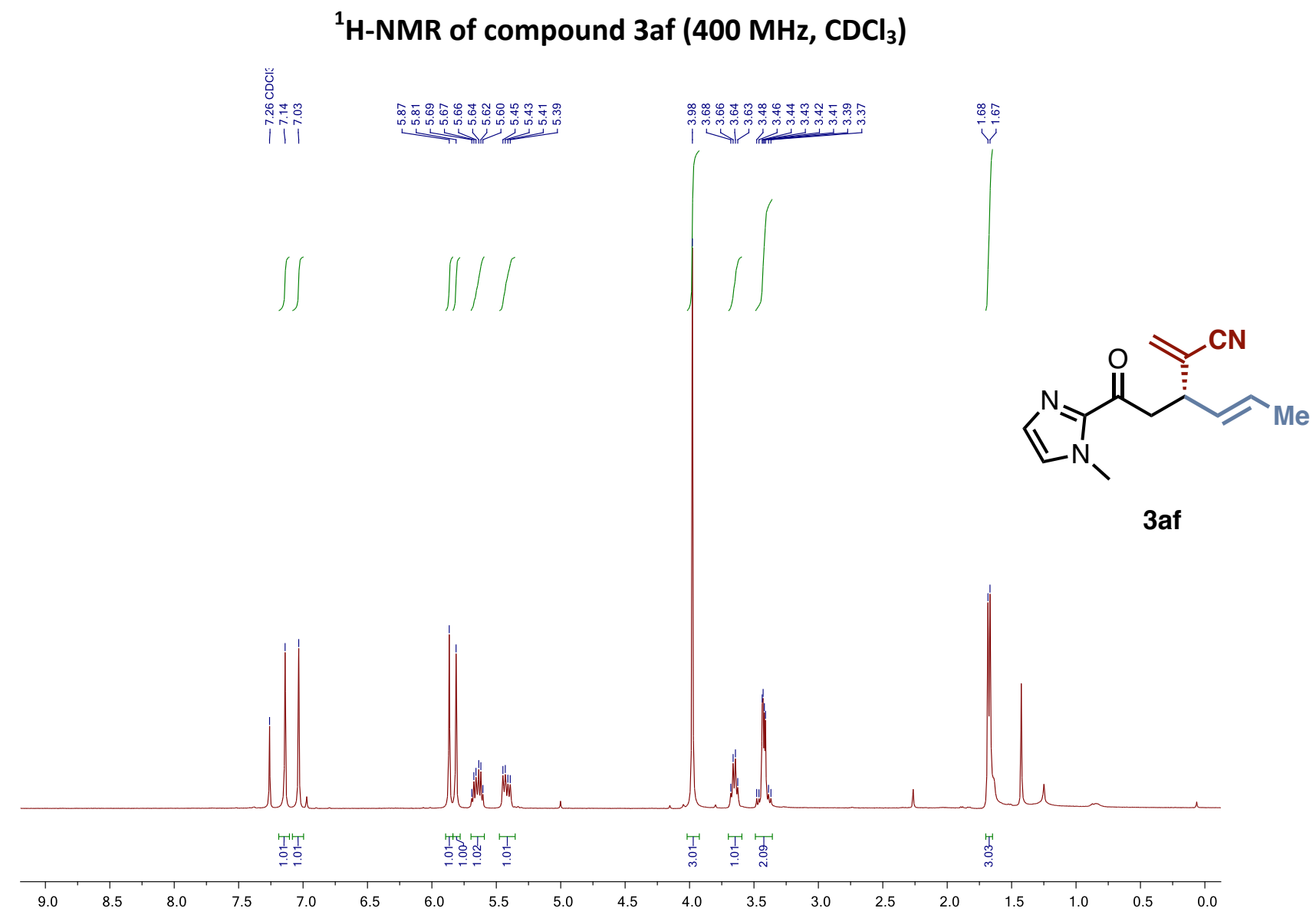

${ }^{13} \mathrm{C}-\mathrm{NMR}$ of compound $3 a f\left(101 \mathrm{MHz}, \mathrm{CDCl}_{3}\right)$

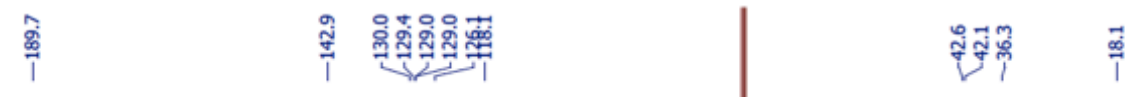

$\begin{array}{llllllllllllllllllllllllllll}210 & 200 & 190 & 180 & 170 & 160 & 150 & 140 & 130 & 120 & 110 & 100 & 90 & 80 & 70 & 60 & 50 & 40 & 30 & 20 & 10 & 0 & -10\end{array}$ 

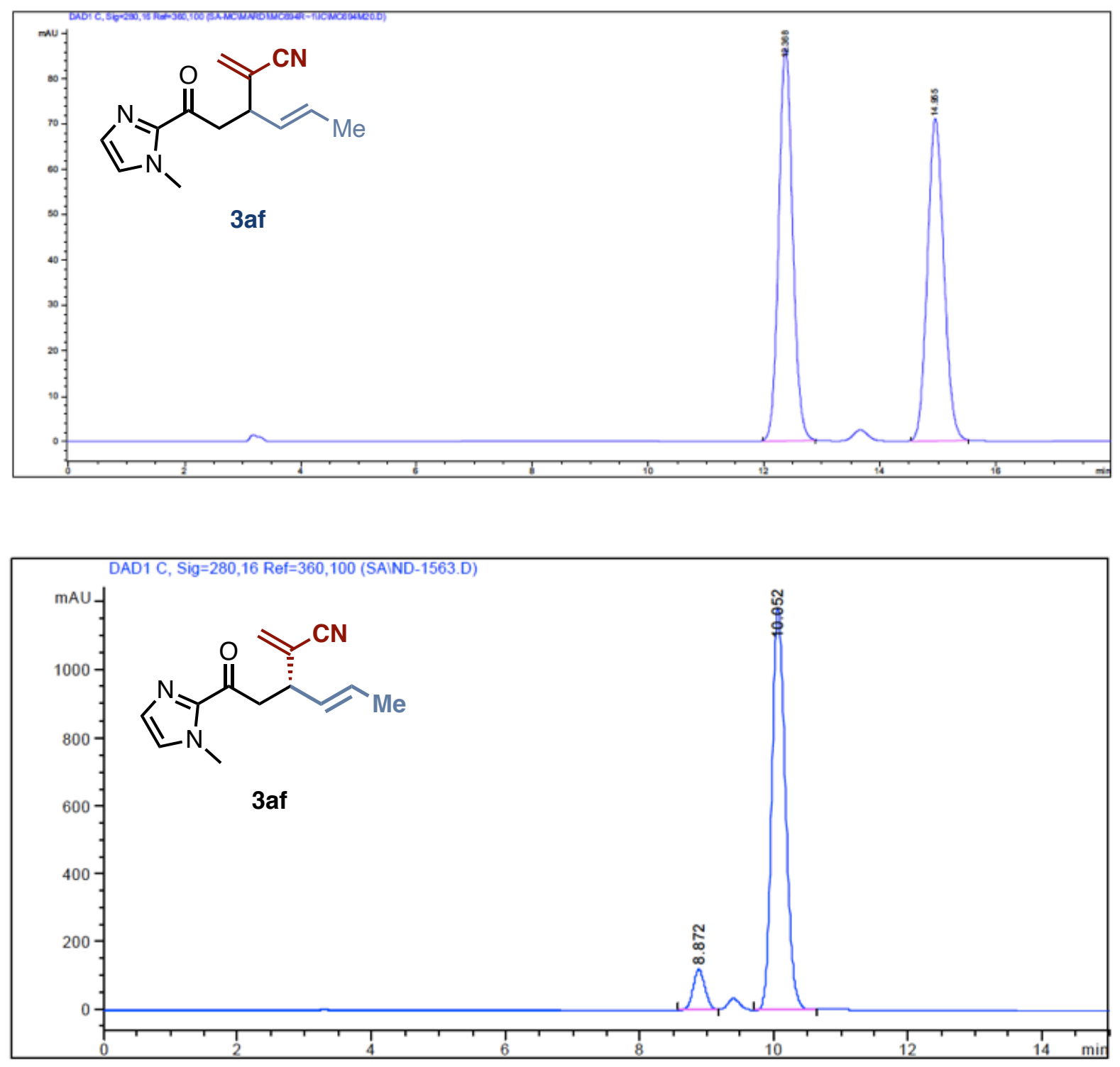

\begin{tabular}{|c|c|c|c|c|c|c|}
\hline $\begin{array}{c}\text { eak } \\
\#\end{array}$ & $\begin{array}{c}\text { RetTime } \\
\text { [min] }\end{array}$ & Type & $\begin{array}{l}\text { Width } \\
\text { [min] }\end{array}$ & $\begin{array}{c}\text { Area } \\
{\left[\mathrm{mAU}{ }^{\star} \mathrm{s}\right]}\end{array}$ & $\begin{array}{l}\text { Height } \\
\text { [mAU] }\end{array}$ & $\begin{array}{c}\text { Area } \\
\quad 8\end{array}$ \\
\hline & & & & 08.34 & & 504 \\
\hline & 10.052 & & 0.2189 & $1.67515 \mathrm{e} 4$ & 1185.17310 & 91.7396 \\
\hline
\end{tabular}


Benzyl 4-cyano-3-methylpent-4-enoate (4)

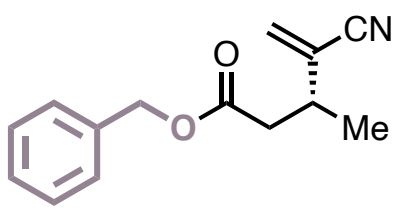

Molecular formula: $\mathrm{C}_{14} \mathrm{H}_{15} \mathrm{NO}_{2}$

$\mathrm{MW}=229.29 \mathrm{~g} \cdot \mathrm{mol}^{-1}$

To a solution of 3-methyl-5-(1-methyl-1H-imidazol-2-yl)-2-methylene-5-oxopentanenitrile 3a (30 mg, $0.15 \mathrm{mmol}, 1$ equiv.) in dry DCM $(1.5 \mathrm{~mL})$ was added methyl trifluoromethanesulfonate $\left(26 \mu \mathrm{L}, 0.225 \mathrm{mmol}, 1.5\right.$ equiv.) at $0{ }^{\circ} \mathrm{C}$ under argon. Upon completion (1 h), Benzyl alcohol $(250 \mu \mathrm{L})$ and DBU $(250 \mu \mathrm{L})$ were slowly added successively. The resulting mixture was stirred at $\mathrm{rt}$ for $30 \mathrm{~min}$, quenched with a saturated aqueous solution of $\mathrm{NaHCO}_{3}(1 \mathrm{~mL})$ and the aqueous phase was extracted with DCM $(2 \times 10 \mathrm{~mL})$. The combined organic layers were concentrated and the resulting crude was purified using flash column chromatography over silica gel $\left(\mathrm{H} / \mathrm{Et}_{2} \mathrm{O}\right.$ 7:3). The title compound was isolated as a colourless oil ( $35 \mathrm{mg}, 0.122 \mathrm{mmol}, 81 \%, 94 \%$ ee).

${ }^{1} \mathbf{H}$ NMR $\left(400 \mathrm{MHz}, \mathrm{CDCl}_{3}\right) \delta$ 7.39-7.31 (m, 5H), $5.80(\mathrm{~s}, 1 \mathrm{H}), 5.75(\mathrm{~d}, J=0.9 \mathrm{~Hz}, 1 \mathrm{H}), 5.13$ (s, 2H), $2.94(\mathrm{~h}, J=7.1 \mathrm{~Hz}, 1 \mathrm{H}), 2.61(\mathrm{dd}, J=15.8,7.6 \mathrm{~Hz}, 1 \mathrm{H}), 2.47(\mathrm{dd}, J=15.8,7.0 \mathrm{~Hz}, 1 \mathrm{H})$, 1.23-1.19 (m, 3H).

${ }^{13} \mathrm{C}$ NMR $\left(101 \mathrm{MHz}, \mathrm{CDCl}_{3}\right) \delta 170.9,135.6,129.6,128.6,128.4,127.0,117.2,66.6,39.6,35.7$, $31.6,22.7,19.1,14.1$.

HRMS (ESI) m/z: [M+H] calcd for $\mathrm{C}_{14} \mathrm{H}_{16} \mathrm{NO}_{2}$ 230.1181; found: 230.1191 .

IR $\left(\mathrm{cm}^{-1}\right): 2970,2937,2224,1736,1458,1285,1261,1168$.

$[a]_{D}^{20}=-30.7\left(c 0.90, \mathrm{CH}_{2} \mathrm{Cl}_{2}\right)$

HPLC analysis: carried out using a mixture of hexane and isopropyl alcohol (97:3 isocratic), at $1 \mathrm{~mL} \cdot \mathrm{min}^{-1}$, at $30{ }^{\circ} \mathrm{C}$ on an ID column, with UV detection $(\lambda=210 \mathrm{~nm})$. Retention times: 12.10 and $12.77 \mathrm{~min}$. 


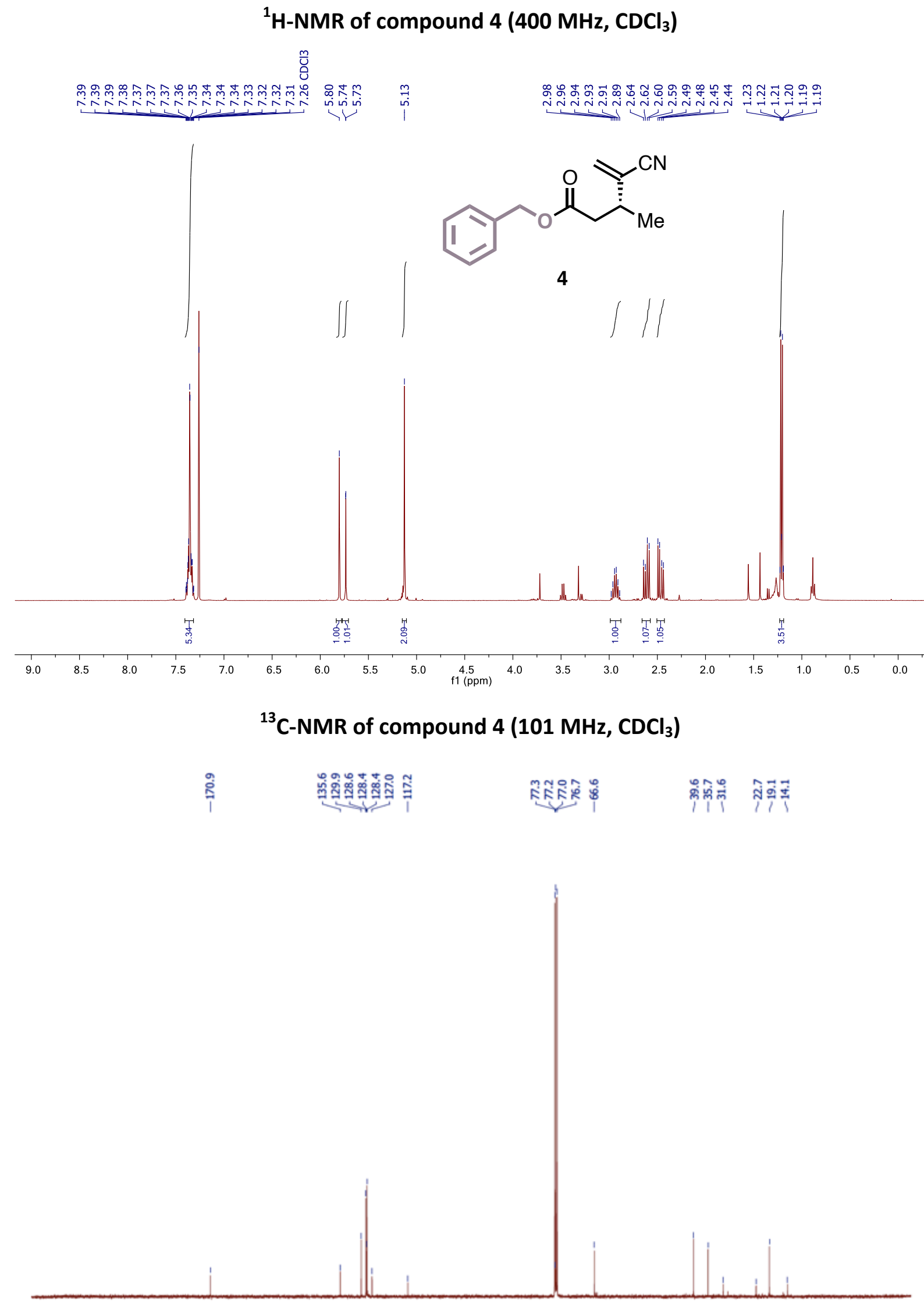

$\begin{array}{lllllllllllllllllllllllllllllllll}210 & 200 & 190 & 180 & 170 & 160 & 150 & 140 & 130 & 120 & 110 & 100 & 90 & 80 & 70 & 60 & 50 & 40 & 30 & 20 & 10 & 0 & -10\end{array}$ 

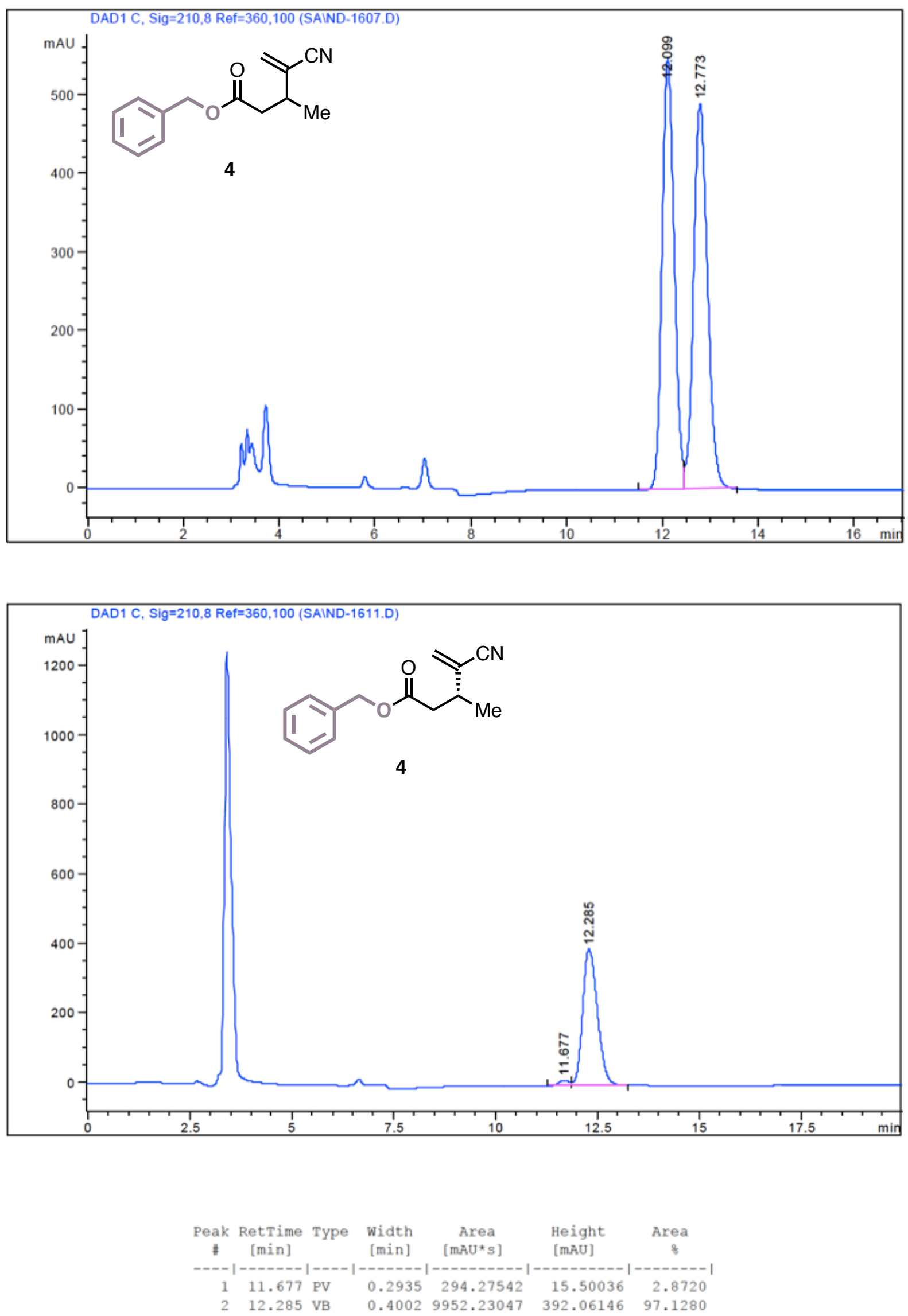
3-Methyl-2-methylene-5-morpholino-5-oxopentanenitrile (5)

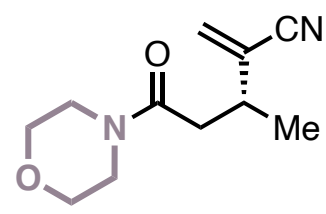

Molecular formula: $\mathrm{C}_{11} \mathrm{H}_{16} \mathrm{~N}_{2} \mathrm{O}_{2}$

$\mathbf{M W}=208.26 \mathrm{~g} \cdot \mathrm{mol}^{-1}$

To a solution of 3-methyl-5-(1-methyl-1H-imidazol-2-yl)-2-methylene-5-oxopentanenitrile 3a (30 mg, $0.15 \mathrm{mmol}, 1$ equiv.) in dry DCM $(1.5 \mathrm{~mL})$ was added methyl trifluoromethanesulfonate (26 $\mathrm{LL}, 0.225 \mathrm{mmol}, 1.5$ equiv.) at $0{ }^{\circ} \mathrm{C}$ under argon. Upon completion $(1 \mathrm{~h})$, morpholine $(600 \mu \mathrm{L})$ was slowly added. The resulting mixture was stirred at RT for $3 \mathrm{~h}$, quenched with a saturated aqueous solution of $\mathrm{NaHCO}_{3}(1 \mathrm{~mL})$ and the aqueous phase was extracted with DCM $(2 \times 10 \mathrm{~mL})$. The combined organic layers were concentrated and the resulting crude was purified using flash column chromatography over silica gel $(\mathrm{Hex} / \mathrm{EtOAc}=5: 5)$. The title compound was isolated as a colourless oil $(20 \mathrm{mg}$, $0.096 \mathrm{mmol}, 64 \%, 95 \%$ ee).

${ }^{1}$ H NMR $\left(400 \mathrm{MHz}, \mathrm{CDCl}_{3}\right) \delta 5.85(\mathrm{~s}, 1 \mathrm{H}), 5.85(\mathrm{~s}, 1 \mathrm{H}), 3.69-367(\mathrm{~m}, 4 \mathrm{H}), 3.64-3.54(\mathrm{~m}, 2 \mathrm{H})$, $3.49(\mathrm{dd}, J=10.0,4.8 \mathrm{~Hz}, 2 \mathrm{H}), 3.09(\mathrm{~h}, J=6.9 \mathrm{~Hz}, 1 \mathrm{H}), 2.58(\mathrm{dd}, J=15.8,7.3 \mathrm{~Hz}, 1 \mathrm{H}), 2.35$ (dd, $J=15.8,6.6 \mathrm{~Hz}, 1 \mathrm{H}), 1.22(\mathrm{~d}, J=6.9 \mathrm{~Hz} 3 \mathrm{H})$.

${ }^{13} \mathrm{C}$ NMR $\left(101 \mathrm{MHz}, \mathrm{CDCl}_{3}\right) \delta 168.8,130.3,127.4,117.5,66.9,66.6,46.0,42.1,37.7,35.8$, 19.5.

HRMS (ESI) m/z: [M+H] calcd for $\mathrm{C}_{11} \mathrm{H}_{17} \mathrm{~N}_{2} \mathrm{O}_{2}$ 209.1290; found: 209.1285 .

IR (cm $\left.{ }^{-1}\right): 2967,2926,2857,2224,1734,1641,1460,1438,1276,1233,1117$.

$[a]_{D}^{20}=-30.7\left(c 0.90, \mathrm{CH}_{2} \mathrm{Cl}_{2}\right)$

HPLC analysis: carried out using a mixture of hexane and isopropyl alcohol (80:20 isocratic), at $1 \mathrm{~mL} \cdot \mathrm{min}^{-1}$, at $30{ }^{\circ} \mathrm{C}$ on an ID column, with UV detection $(\lambda=210 \mathrm{~nm})$. Retention times: 16.27 and $17.89 \mathrm{~min}$. 
${ }^{1} \mathrm{H}-\mathrm{NMR}$ of compound $5\left(400 \mathrm{MHz}, \mathrm{CDCl}_{3}\right)$<smiles>C=C(C#N)[C@H](C)CC(=O)N1CCOCC1</smiles>

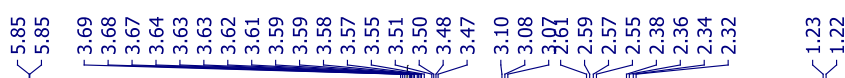

5

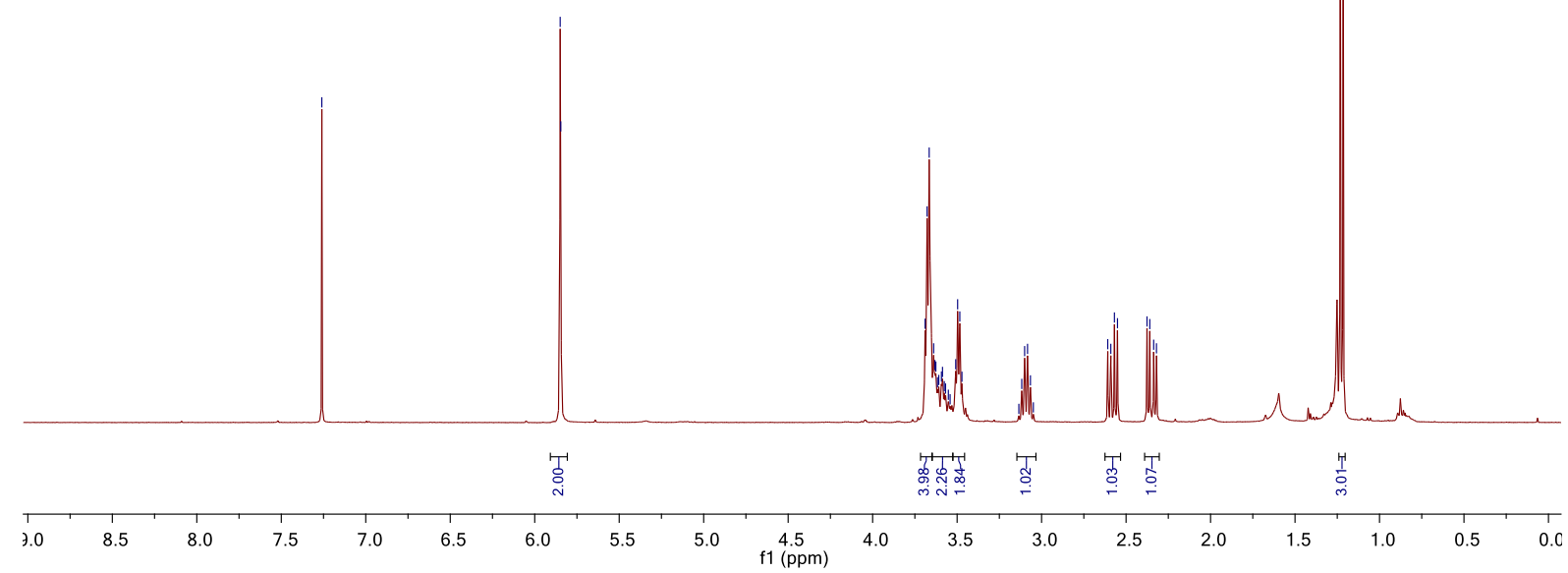

${ }^{13} \mathrm{C}-\mathrm{NMR}$ of compound $5\left(101 \mathrm{MHz}, \mathrm{CDCl}_{3}\right)$

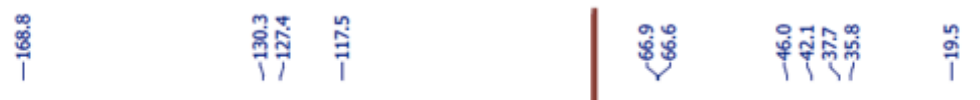

$\begin{array}{lllllllllllllllllllllll}210 & 200 & 190 & 180 & 170 & 160 & 150 & 140 & 130 & 120 & 110 & 100 & 90 & 80 & 70 & 60 & 50 & 40 & 30 & 20 & 10 & 0 & -10\end{array}$ 

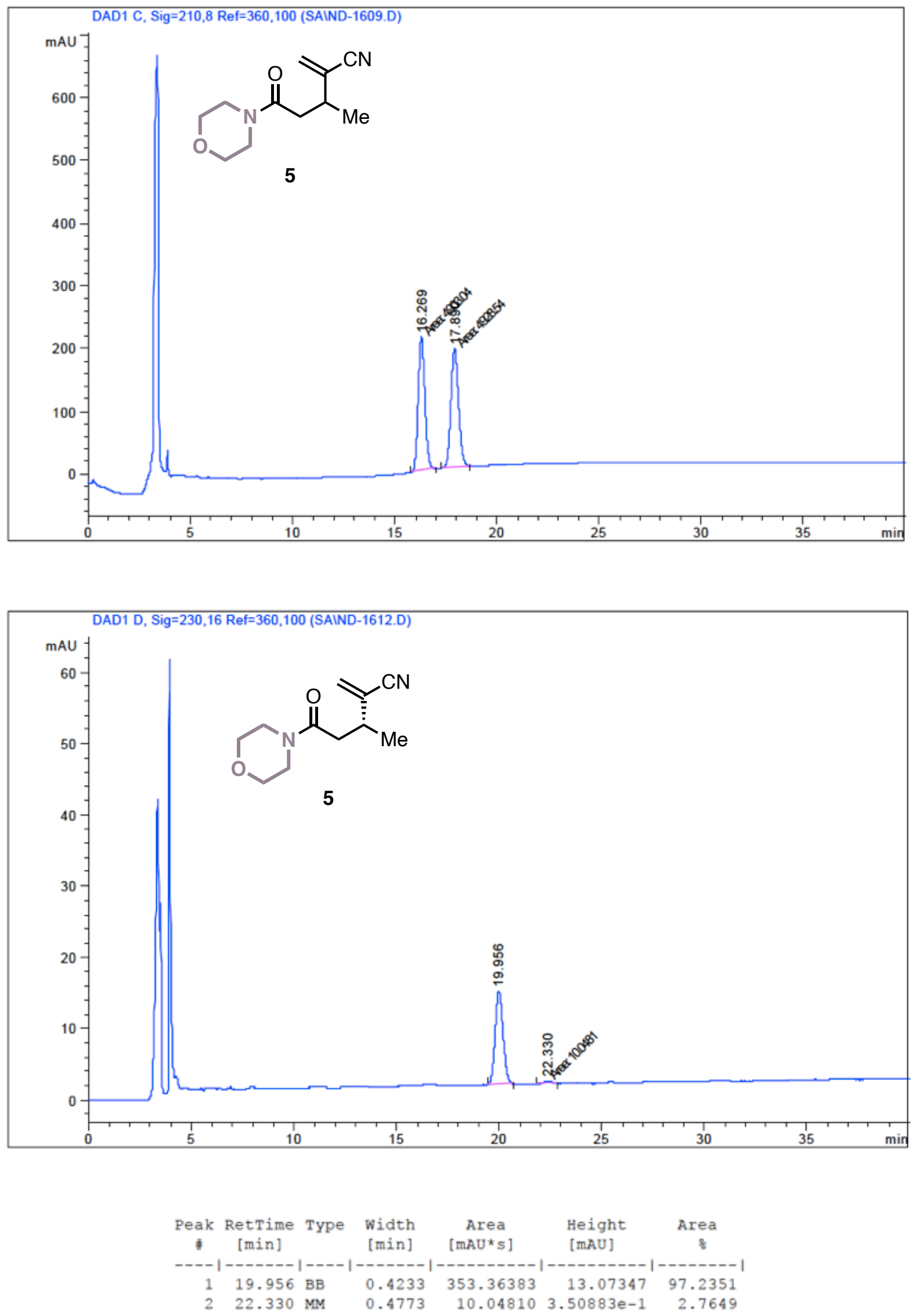


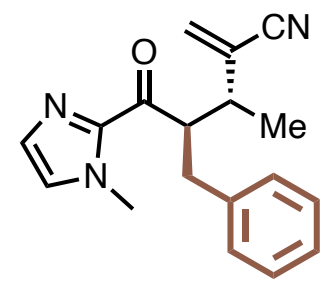

Molecular formula: $\mathrm{C}_{18} \mathrm{H}_{19} \mathrm{~N}_{3} \mathrm{O}$

$\mathrm{MW}=293.37 \mathrm{~g} \cdot \mathrm{mol}^{-1}$

To a solution of 3-methyl-5-(1-methyl-1H-imidazol-2-yl)-2-methylene-5-oxopentanenitrile $3 a$ (30 mg, $0.15 \mathrm{mmol}, 1$ equiv.) and tetrabutylammonium bromide in dry DCM (1.5 mL) was added benzyl bromide ( $89 \mu \mathrm{L}, 0.75 \mathrm{mmol}, 5$ equiv.) at $0{ }^{\circ} \mathrm{C}$ under argon. Cesium hydroxide hydrate (100 mg, $0.60 \mathrm{mmol}$, i4 equiv.) was then added, the resulting solution was allowed to warm up to rt was stirred vigorously overnight. Upon completion the reaction mixture was quenched $\mathrm{H}_{2} \mathrm{O}(5 \mathrm{~mL})$ and the aqueous phase was extracted with $\mathrm{DCM}(2 \times 10 \mathrm{~mL})$. The combined organic layers were concentrated and the resulting crude was purified using flash column chromatography over silica gel $(\mathrm{Hex} /$ EtOAc $=7: 3)$. The title compound was isolated as a colourless oil as a mixture of both diastereoisomers $(35 \mathrm{mg}, 0.120 \mathrm{mmol}, 80 \%, \mathrm{dr}=7: 2$, 92\% ee on both).

${ }^{1} \mathrm{H}$ NMR $\left(400 \mathrm{MHz}, \mathrm{CDCl}_{3}\right) \delta 7.19-7.05(\mathrm{~m}, 6 \mathrm{H}+1.8 \mathrm{H}), 6.91(\mathrm{~s}, 1 \mathrm{H}+0.3 \mathrm{H}), 5.93(\mathrm{~s}, 0.3 \mathrm{H}$, dia $\min$ ), $5.85(\mathrm{~s}, 1 \mathrm{H}$, dia maj), $5.84(\mathrm{~s}, 0.3 \mathrm{H}$, dia $\min ), 5.78(\mathrm{~d}, \mathrm{~J}=0.8 \mathrm{~Hz}, 1 \mathrm{H}$, dia maj), 4.46 (ddd, $J=10.2,7.1,4.7 \mathrm{~Hz}, 1 \mathrm{H}$, dia maj), 4.38 (td, $J=8.7,6.7 \mathrm{~Hz}, 0.3 \mathrm{H}$, dia min), $3.84(\mathrm{~s}, 3 \mathrm{H}$, dia maj), $3.83(\mathrm{~s}, 1.0 \mathrm{H}$, dia min), 3.09-2.92 (m, 3H + 1H), $1.27(\mathrm{~d}, \mathrm{~J}=7.1 \mathrm{~Hz}, 3 \mathrm{H}$, dia maj), 1.19 (d, $J=6.9 \mathrm{~Hz}, 1.0 \mathrm{H}$, dia $\min$ ).

${ }^{13} \mathrm{C}$ NMR (101 MHz, $\mathrm{CDCl}_{3}$ ) $\delta 194.0$ (dia min), 193.2 (dia maj), 143.7 (dia min), 143.2 (dia maj), 138.8 (dia maj), 138.4 (dia min), 131.1 (dia min), 130.7 (dia maj), 129.3 (dia min), 129.3 (dia maj), 129.1 (dia maj), 129.0 (dia min), 128.2 (dia maj), 128.1 (dia min), 127.2 (dia min), 127.1 (dia maj), 126.8 (dia min), 126.2 (dia maj), 117.9 (dia maj), 52.0 (dia maj), 51.9 (dia $\mathrm{min}$ ), 42.1 (dia $\mathrm{min}$ ), 40.3 (dia maj), 36.8 (dia $\mathrm{min}$ ), 36.0 (dia maj), 36.0 (dia min), 34.3 (dia maj), 22.7 (dia min), 18.1 (dia maj), 16.0 (dia maj), 14.1 (dia min).

HRMS (ESI) m/z: [M+Na] $]^{+}$calcd for $\mathrm{C}_{18} \mathrm{H}_{19} \mathrm{~N}_{3} \mathrm{ONa} 316.1426$; found: 316.1429.

IR $\left(\mathrm{cm}^{-1}\right):$ 2969, 2933, 2224, 1670, 1458, 1408, 1292, 1158, 946, 914. 
$[\boldsymbol{a}]_{\boldsymbol{D}}^{\mathbf{2 0}}=-94.5\left(c\right.$ 0.80, $\left.\mathrm{CH}_{2} \mathrm{Cl}_{2}\right)$

HPLC analysis: carried out using a mixture of hexane and isopropyl alcohol (95:5 isocratic) at $1 \mathrm{~mL} \cdot \mathrm{min}^{-1}$, at $30^{\circ} \mathrm{C}$ on a IC columns, with UV detection $(\lambda=280 \mathrm{~nm})$. Retention times: 28.66 and $31.15 \mathrm{~min}$ (dia maj), 21.95 and $34.02 \mathrm{~min}$ (dia $\mathrm{min}$ ). 
${ }^{1} \mathrm{H}-\mathrm{NMR}$ of compound $6\left(400 \mathrm{MHz}, \mathrm{CDCl}_{3}\right)$

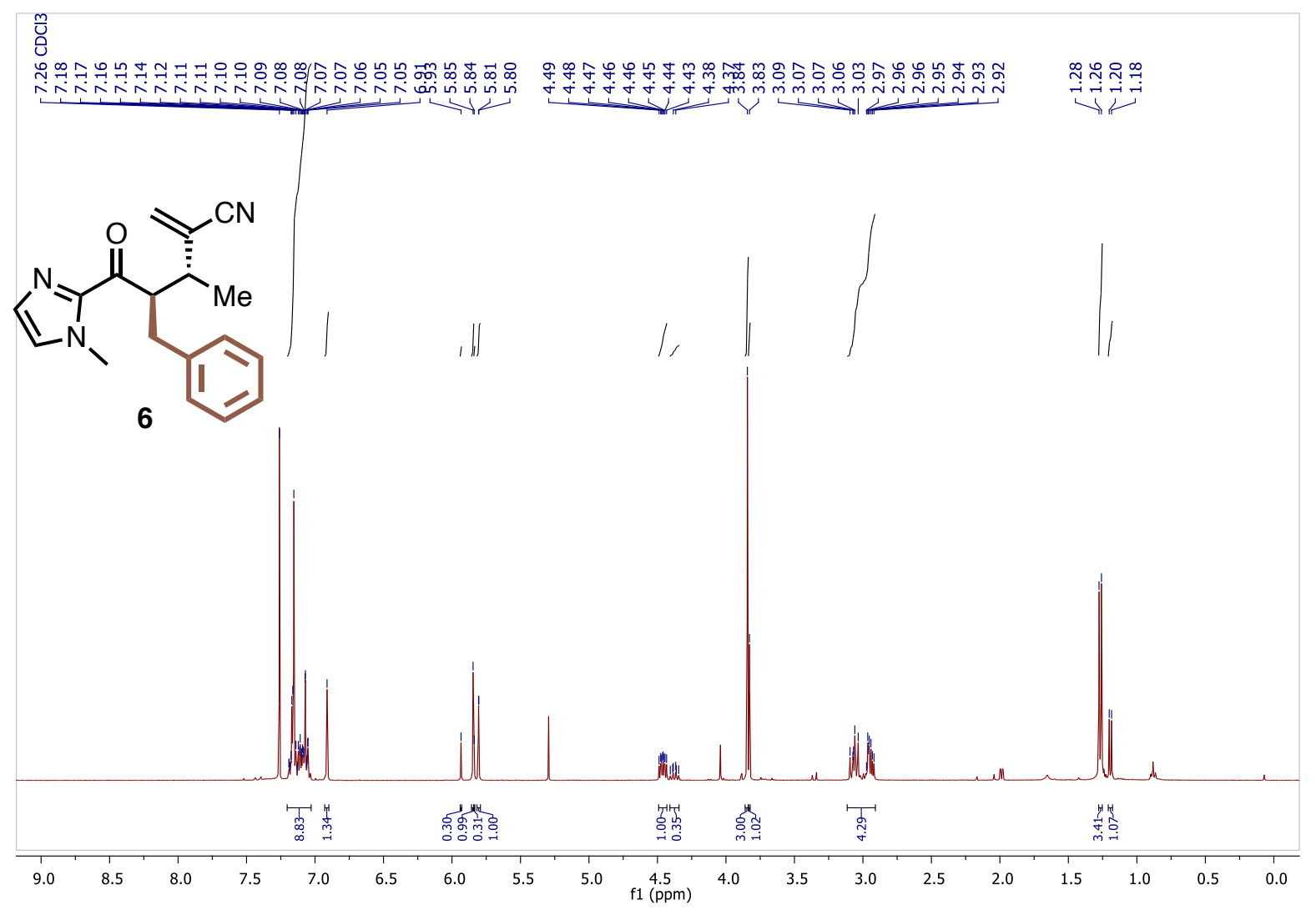

${ }^{13} \mathrm{C}-\mathrm{NMR}$ of compound $6\left(101 \mathrm{MHz}, \mathrm{CDCl}_{3}\right)$

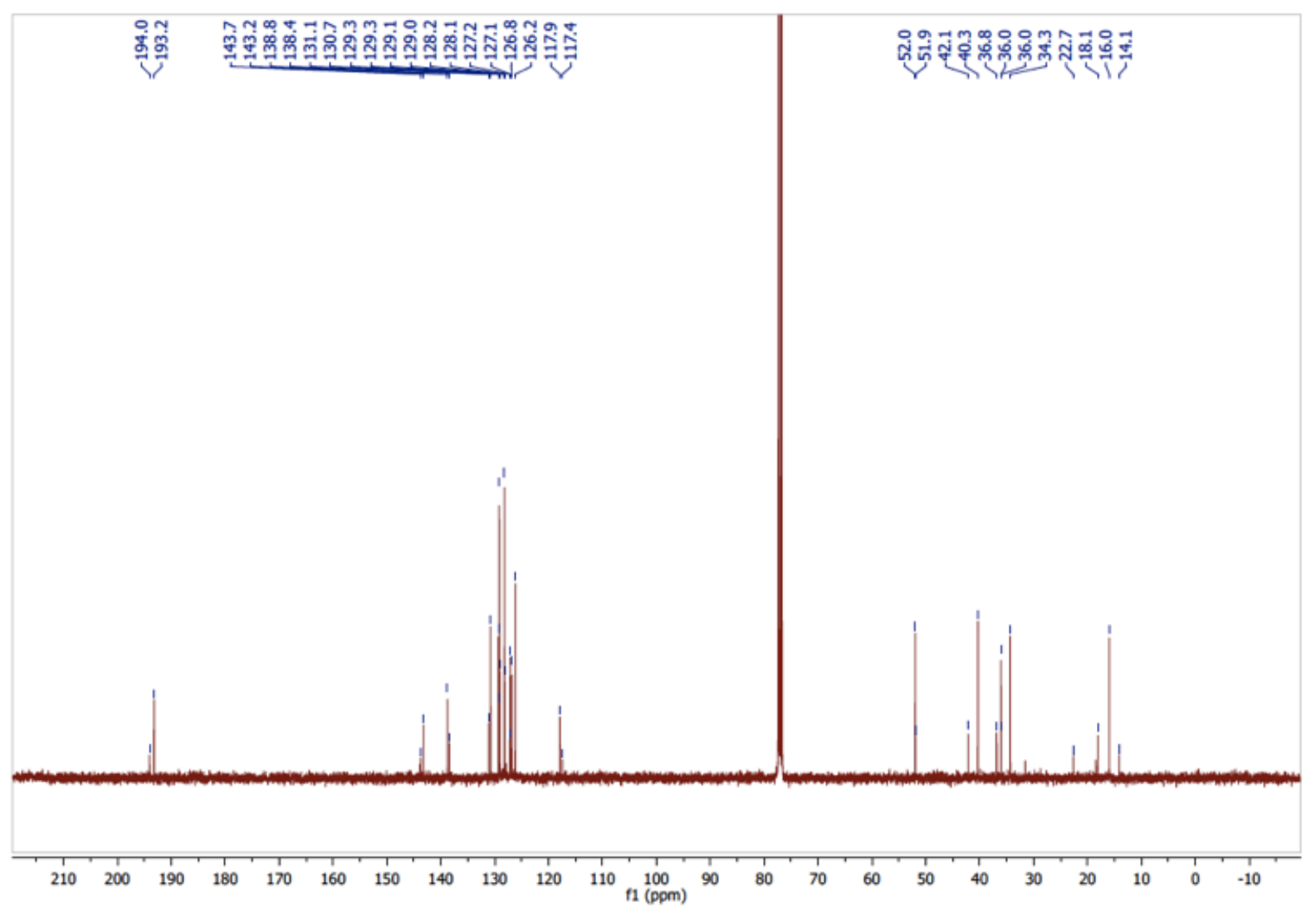



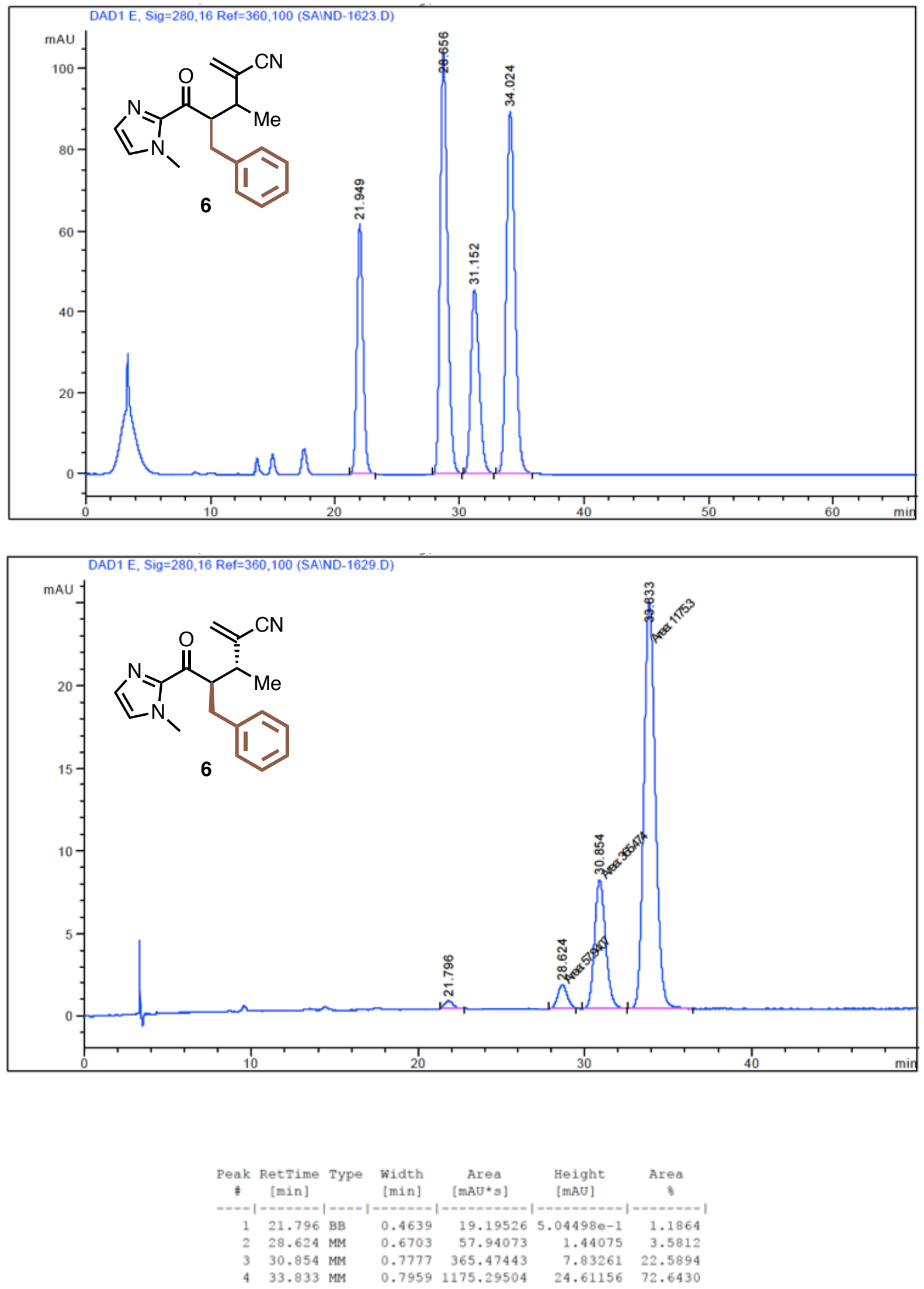
3-Methyl-4-(1-methyl-1H-imidazole-2-carbonyl)-2-methylenehept-6-enenitrile (7)

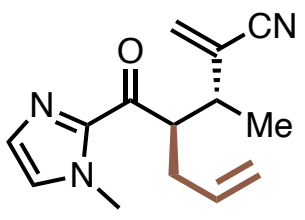

Molecular formula: $\mathrm{C}_{14} \mathrm{H}_{17} \mathrm{~N}_{3} \mathrm{O}$

$\mathrm{MW}=243.31 \mathrm{~g} \cdot \mathrm{mol}^{-1}$

To a solution of 3-methyl-5-(1-methyl-1H-imidazol-2-yl)-2-methylene-5-oxopentanenitrile (30 mg, $0.15 \mathrm{mmol}, 1$ equiv.) and tetrabutylammonium bromide in dry DCM (1.5 mL) was added allyl bromide (90 $\mu \mathrm{L}, 0.75 \mathrm{mmol}, 5$ equiv.) at $0{ }^{\circ} \mathrm{C}$ under argon. Cesium hydroxide hydrate (100 mg, $0.60 \mathrm{mmol}, 4$ equiv.) was then added, the resulting solution was allowed to warm up to rt was stirred vigorously overnight. Upon completion the reaction mixture was quenched $\mathrm{H}_{2} \mathrm{O}(5 \mathrm{~mL})$ and the aqueous phase was extracted with DCM $(2 \times 10 \mathrm{~mL})$. The combined organic layers were concentrated and the resulting crude was purified using flash column chromatography over silica gel (Hex/Acetone $=7: 3$ ). The title compound was as colourless oil as a mixture of both diastereoisomers $(28 \mathrm{mg}, 0.112 \mathrm{mmol}, 77 \%, \mathrm{dr}=7: 3$, both at $94 \% e e)$.

${ }^{1} \mathrm{H}$ NMR $\left(400 \mathrm{MHz}, \mathrm{CDCl}_{3}\right) \delta 7.17(\mathrm{~d}, J=0.8 \mathrm{~Hz}, 0.4 \mathrm{H}$, dia $\min ), 7.16(\mathrm{~d}, J=0.8 \mathrm{~Hz}, 1 \mathrm{H}$, dia maj), 7.04 (s, 0.4H, dia min), 7.02 (s, $1 \mathrm{H}$, dia majo), 5.90 (s, 0.4H, dia min), 5.80 (s, 0.4H, dia min), $5.78(\mathrm{~s}, 1 \mathrm{H}, \operatorname{dia}$ maj), $5.76(\mathrm{~s}, 1 \mathrm{H}$, dia maj), 5.75-5.61 (m, 1H + 0.4H), 5.02 (ddd, J = 17.1, 3.1, $1.6 \mathrm{~Hz}, 1 \mathrm{H}$, dia maj), 4.97-4.89 (m, $1 \mathrm{H}+0.8 \mathrm{H}), 4.20$ (td, J = 8.7, $4.4 \mathrm{~Hz}, 1 \mathrm{H}$, dia maj), 4.15 (m, 0.4H, dia $\min ), 3.98(\mathrm{~s}, 1.2 \mathrm{H}$, dia $\min ), 3.96(\mathrm{~s}, 3 \mathrm{H}, \operatorname{dia} \operatorname{maj}), 2.99-2.87(\mathrm{~m}, 1 \mathrm{H}+0.4 \mathrm{H})$, 2.57-2.41 (m, 2H + 0.8H), $1.23(\mathrm{~d}, J=7.0 \mathrm{~Hz}, 3 \mathrm{H}$, dia maj), $1.14(\mathrm{~d}, J=6.8 \mathrm{~Hz}, 1.2 \mathrm{H}$, dia min).

${ }^{13} \mathrm{C}$ NMR (101 MHz, $\mathrm{CDCl}_{3}$ ) $\delta 194.0$ (dia min), 193.4 (dia maj), 143.6 (dia min), 143.1 (dia maj), 134.6 (dia maj), 134.5 (dia min), 131.0 (dia min), 130.6 (dia maj), 129.5 (dia min), 129.5 (dia maj), 127.5 (dia min), 127.4 (dia maj), 127.0 (dia maj), 126.7 (dia min), 117.8 (dia maj), 117.4 (dia min), 117.2 (dia maj), 117.1 (dia min), 49.7 (dia maj), 49.5 (dia min), 41.2 (dia min), 39.4 (dia maj), 36.2 (dia maj), 36.2 (dia min), 35.1 (dia min), 33.2 (dia maj), 18.1 (dia $\min$ ), 16.2 (dia maj).

HRMS (ESI) m/z: [M+Na] $]^{+}$calcd for $\mathrm{C}_{14} \mathrm{H}_{17} \mathrm{~N}_{3} \mathrm{ONa} 266.1269$; found: 266.1266.

IR $\left(\mathrm{cm}^{-1}\right):$ 3101, 2972, 2935, 2224, 1730, 1672, 1462, 1408, 1292, 1158. 
$[\boldsymbol{a}]_{\boldsymbol{D}}^{20}=-94.5\left(c 0.80, \mathrm{CH}_{2} \mathrm{Cl}_{2}\right)$

HPLC: was carried out using a mixture of hexane and isopropyl alcohol (97:3 isocratic), at $1 \mathrm{~mL} \cdot \mathrm{min}^{-1}$, at $30{ }^{\circ} \mathrm{C}$ on IA + IC columns, with UV detection $(\lambda=210 \mathrm{~nm})$. Retention times: 49.24 and $58.98 \mathrm{~min}$ (dia maj), 46.44 and $62.62 \mathrm{~min}$ (dia $\mathrm{min}$ ). 
${ }^{1} \mathrm{H}-\mathrm{NMR}$ of compound $7\left(400 \mathrm{MHz}, \mathrm{CDCl}_{3}\right)$

吕

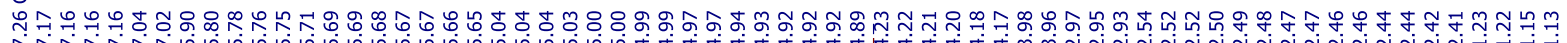

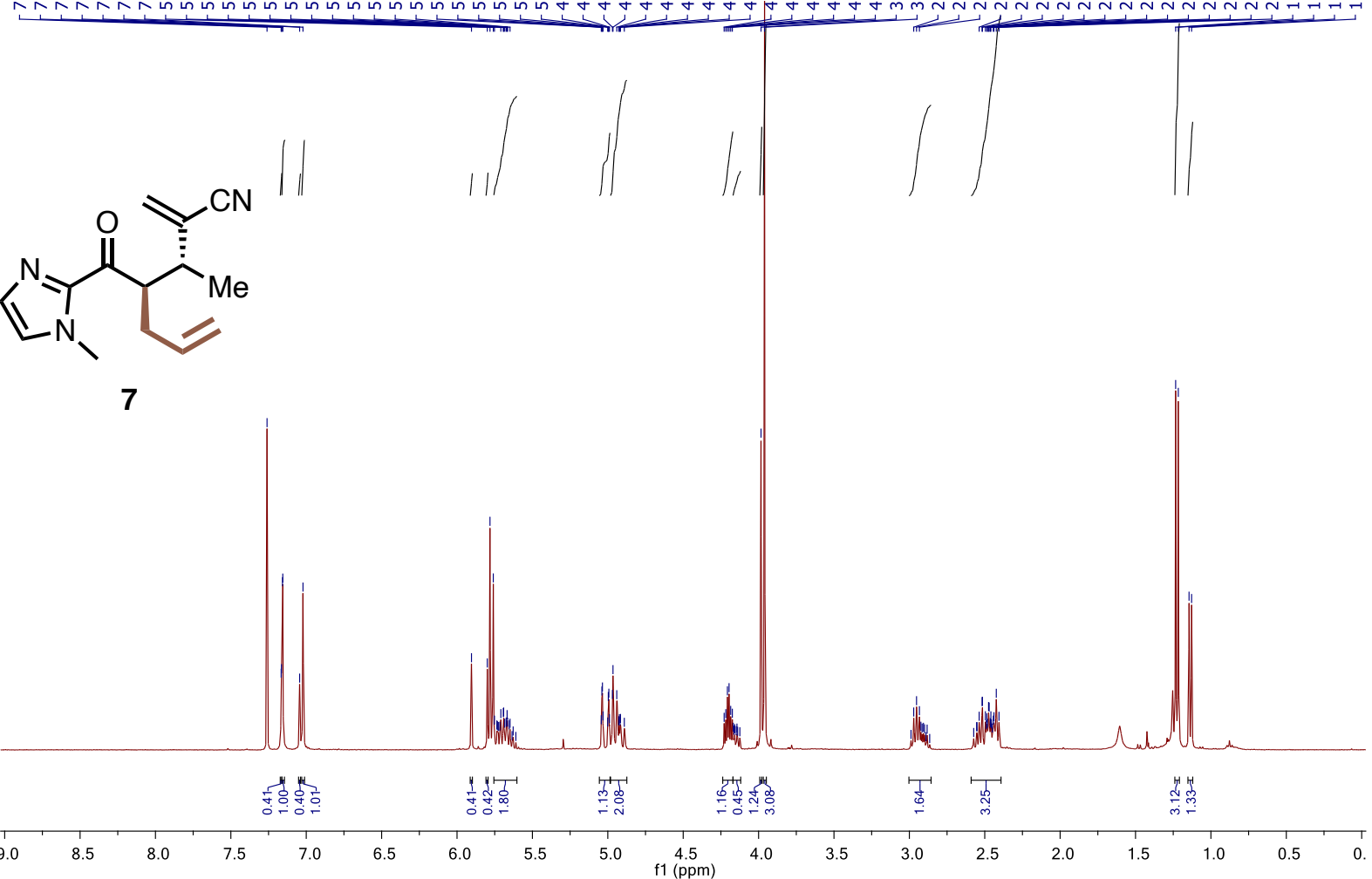

${ }^{13} \mathrm{C}-\mathrm{NMR}$ of compound $7\left(101 \mathrm{MHz}, \mathrm{CDCl}_{3}\right)$

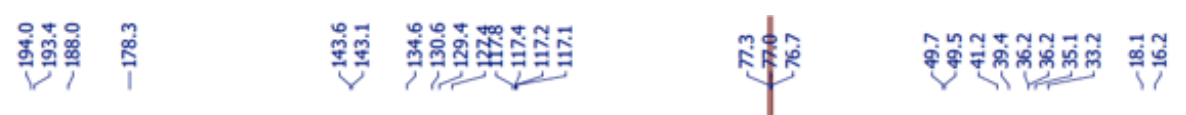

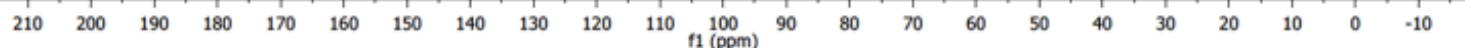



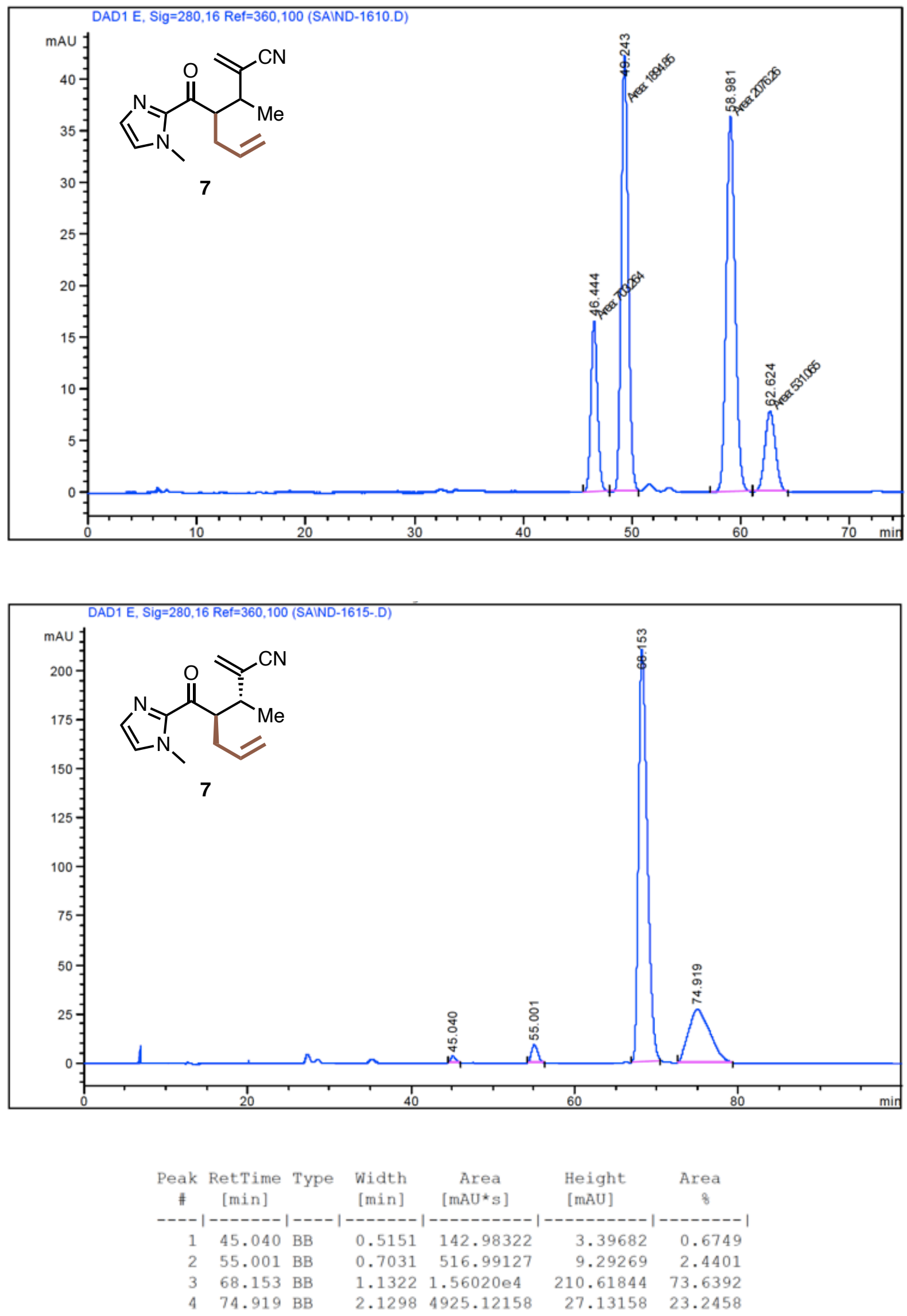
5-Methyl-4-(1-methyl-1H-imidazole-2-carbonyl)cyclopent-1-ene-1-carbonitrile (8)

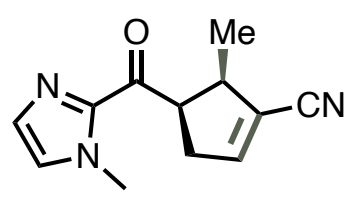

Molecular formula: $\mathrm{C}_{12} \mathrm{H}_{13} \mathrm{~N}_{3} \mathrm{O}$

$\mathbf{M W}=215.26 \mathrm{~g} \cdot \mathrm{mol}^{-1}$

To a solution of 3-Methyl-4-(1-methyl-1H-imidazole-2-carbonyl)-2-methylenehept-6enenitrile 7 (24 mg, 0.1 mmol, 1 equiv.) in dry and degassed DCM (5 mL) was added Grubbs II (4 mg, $0.005 \mathrm{mmol}, 0.05$ equiv.) and the resulting solution warmed up $40{ }^{\circ} \mathrm{C}$ using a heating mantle and stirred overnight. After overnight stirring, the solution was concentrated and purified using flash column chromatography over silica gel $(H / E A / A c e t o n e=6: 3: 1)$. The title compound was isolated as a colourless oil, as a mixture of both diasteroisomers $(9 \mathrm{mg}$, $0.41 \mathrm{mmol}, 41 \%, 65 \%$ (brsm), $\mathrm{dr}=7: 3 \mathrm{95 \%}$ ee on minor enantiomer, $93 \%$ ee on major enantiomer).

${ }^{1} \mathrm{H}$ NMR $\left(400 \mathrm{MHz}, \mathrm{CDCl}_{3}\right) \delta 7.17(\mathrm{~s}, 1 \mathrm{H}+0.4 \mathrm{H}), 7.07$ (s, 0.4H, dia min), 7.06 (s, 1H, dia majo), $6.64(\mathrm{td}, J=2.8,1.4 \mathrm{~Hz}, 1 \mathrm{H}$, dia maj), 6.54 (dd, $J=4.7,2.5 \mathrm{~Hz}, 0.4 \mathrm{H}$, dia $\min$ ), 4.62 (q, $J=8.8 \mathrm{~Hz}, 1 \mathrm{H}$, dia maj), $4.14(\mathrm{~m}, 0.4 \mathrm{H}$, dia $\min ), 4.02(\mathrm{~s}, 3 \mathrm{H}+1.2 \mathrm{H}), 3.60$ (dd, $J=13.7,7.0 \mathrm{~Hz}$, $1 \mathrm{H}$, dia maj), 3.34 (ddd, J = 9.3, 5.0, $2.6 \mathrm{~Hz}, 0.4 \mathrm{H}$, dia min), 3.17 (m, 1H, dia maj), 2.99 (ddt, $J=18.9,9.5,2.8 \mathrm{~Hz}, 0.4 \mathrm{H}$, dia $\min$ ), 2.79 (ddt, $J=18.9,6.7,2.4 \mathrm{~Hz}, 0.4 \mathrm{H}$, dia min), 2.53 (ddd, $J=18.8,8.6,2.9 \mathrm{~Hz}, 1 \mathrm{H}$, dia maj), 1.34 (d, J = 7.0 Hz, dia min), 0.89 (d, J = 7.1 Hz, dia maj).

${ }^{13} \mathrm{C}$ NMR (101 MHz, CDCl 3 ) $\delta 191.8$ (dia min), 190.9 (dia maj), 146.5 (dia maj), 145.6 (dia min), 129.6 (dia maj), 127.6 (dia min), 127.3 (dia maj), 119.2 (dia min), 118.9 (dia maj), 115.9 (dia maj), 115.9 (dia min), 52.6 (dia maj), 48.9 (dia maj), 48.7 (dia min), 45.8 (dia min), 44.7 (dia maj), 36.9 (dia maj), 36.3 (dia $\mathrm{min}$ ), 36.2 (dia maj), 32.9 (dia maj), 29.7 (dia min), 25.7 (dia $\mathrm{min}$ ), 29.7 (dia maj), 19.0 (dia $\mathrm{min}$ ), 14.8 (dia maj).

HRMS (ESI) m/z: [M+H] $]^{+}$calcd for $\mathrm{C}_{12} \mathrm{H}_{14} \mathrm{~N}_{3} \mathrm{O}$ 216.1137; found: 216.1130 .

IR $\left(\mathrm{cm}^{-1}\right):$ 2961, 2928, 2857, 2363, 2343, 2222, 1676, 1460, 1410, 1292, 1160, 1032.

$[\boldsymbol{a}]_{\boldsymbol{D}}^{\mathbf{2 0}}=+95.1\left(c 0.45, \mathrm{CH}_{2} \mathrm{Cl}_{2}\right)$ 
HPLC analysis: carried out using a mixture of hexane and isopropyl alcohol (90:10 isocratic), at $1 \mathrm{~mL} \cdot \mathrm{min}^{-1}$, at $20^{\circ} \mathrm{C}$ on an ID column, with UV detection $(\lambda=280 \mathrm{~nm})$. Retention times: 19.15 and $21.20 \mathrm{~min}$ (minor diastereoisomer), 26.18 and 32.79 min (major diastereoisomer). 
${ }^{1} \mathrm{H}-\mathrm{NMR}$ of compound $8\left(400 \mathrm{MHz}, \mathrm{CDCl}_{3}\right)$

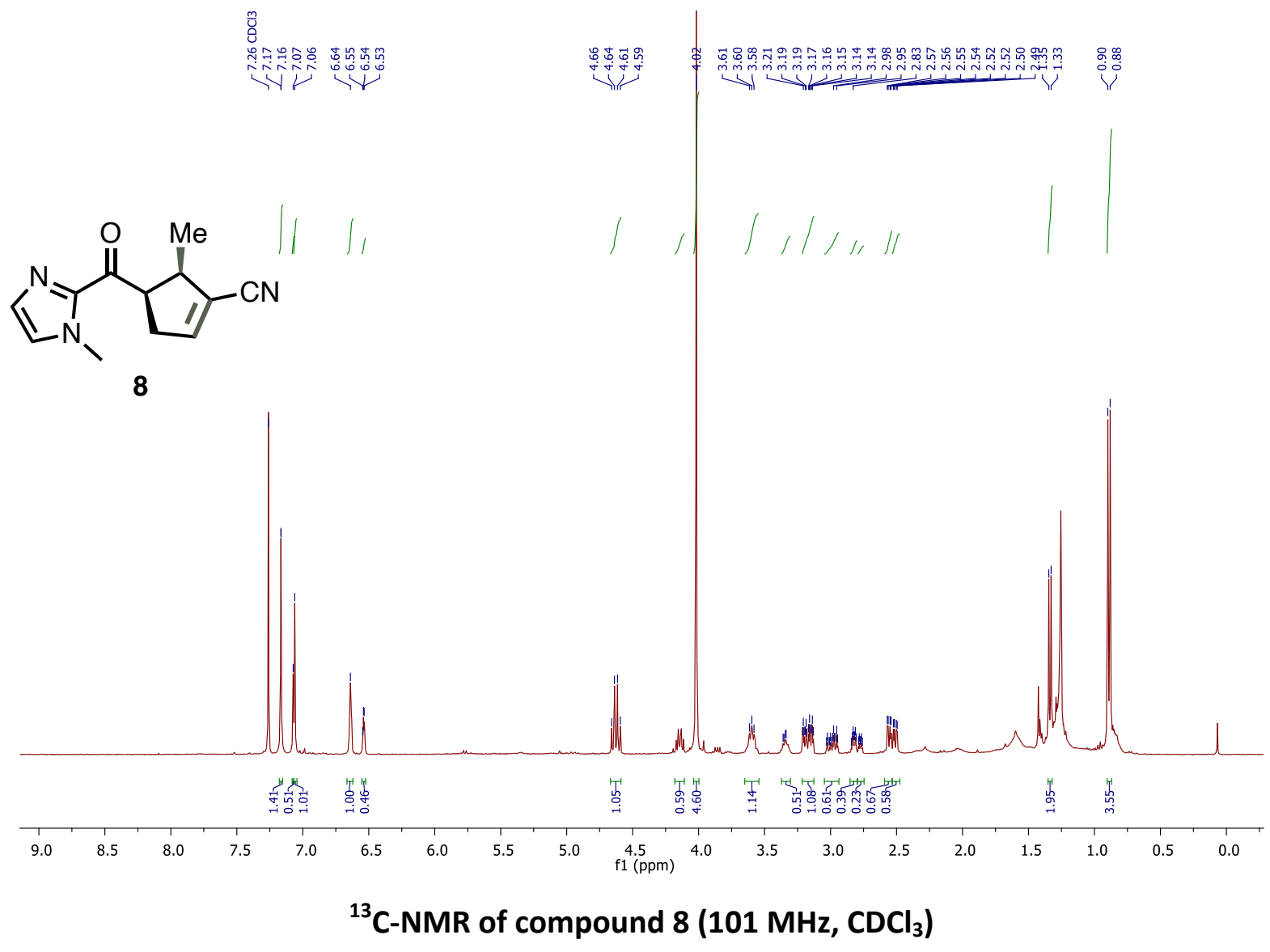

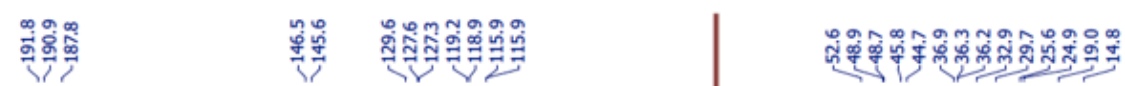

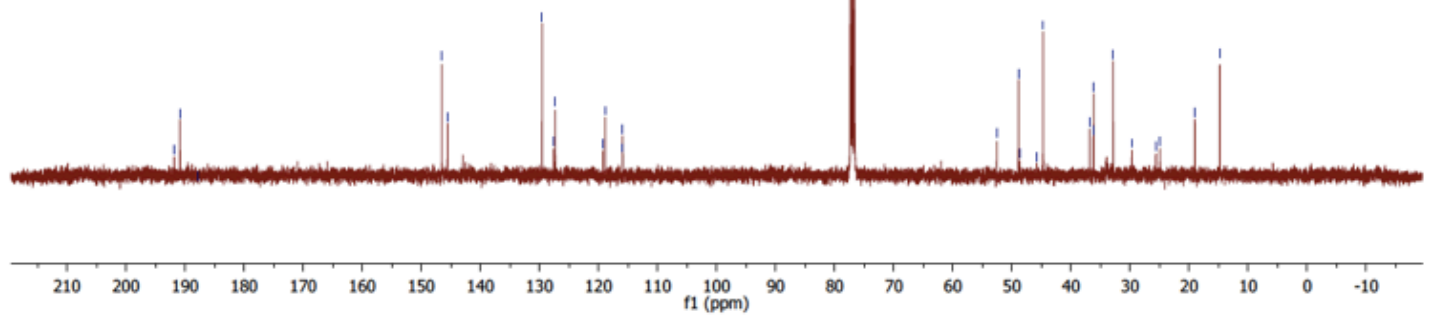



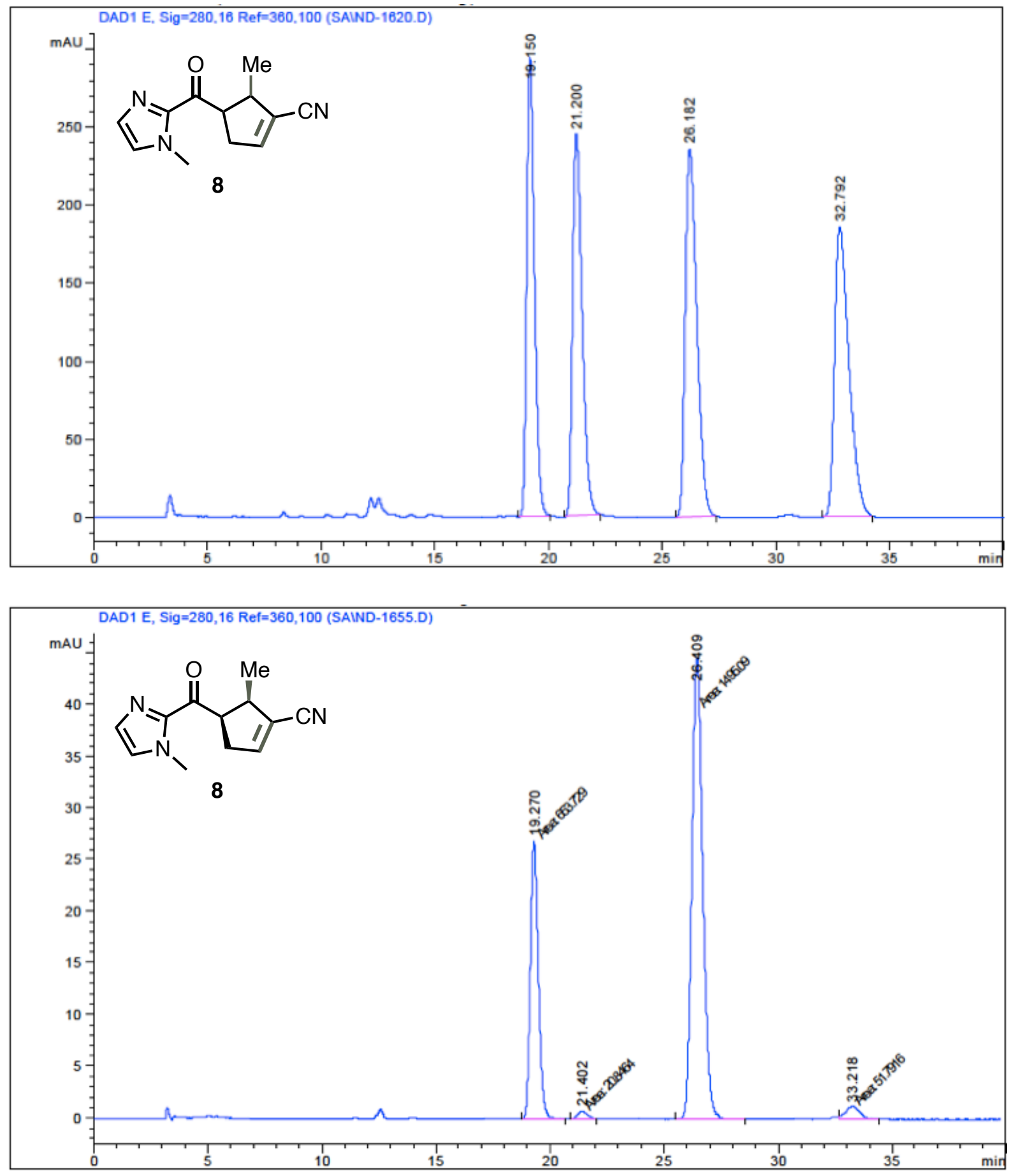

\begin{tabular}{|c|c|c|c|c|c|c|}
\hline $\begin{array}{c}\text { Peak } \\
\neq\end{array}$ & $\begin{array}{c}\text { RetTime } \\
\text { [min] }\end{array}$ & Type & $\begin{array}{c}\text { Width } \\
\text { [min] }\end{array}$ & $\begin{array}{c}\text { Area } \\
{\left[m A U^{*} s\right]}\end{array}$ & $\begin{array}{l}\text { Height } \\
\text { [mAU] }\end{array}$ & $\begin{array}{c}\text { Area } \\
\text { \& }\end{array}$ \\
\hline & & & & |---n--- & & 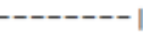 \\
\hline 1 & 19.270 & $\mathrm{MM}$ & 0.4042 & 653.72876 & 26.95738 & 29.4279 \\
\hline 2 & 21.402 & MM & 0.4799 & 20.84643 & $7.23922 \mathrm{e}-1$ & 0.9384 \\
\hline 3 & 26.409 & $M M$ & 0.5579 & 1495.09302 & 44.66772 & 67.3023 \\
\hline 4 & 33.218 & $M A$ & 0.7061 & 51.79156 & 1.22244 & 2.3314 \\
\hline
\end{tabular}




\section{5-[2-(1-Methyl-1H-imidazol-2-yl)-2-oxoethyl]cyclopent-1-ene-1-carbonitrile (9)}

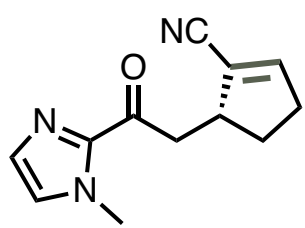

Molecular formula: $\mathrm{C}_{12} \mathrm{H}_{13} \mathrm{~N}_{3} \mathrm{O}$

$\mathbf{M W}=215.26 \mathrm{~g} \cdot \mathrm{mol}^{-1}$

To a solution of 3-(2-(1-methyl-1H-imidazol-2-yl)-2-oxoethyl)-2-methylenehept-6-enenitrile 3 g (33 mg, $0.136 \mathrm{mmol}, 1$ equiv.) in dry and degassed DCM (10 mL) was added Grubbs II (6 mg, $0.007 \mathrm{mmol}, 0.05$ equiv.) and the resulting solution was warmed up to $40{ }^{\circ} \mathrm{C}$ using a heating mantle and stirred overnight. After overnight stirring, the solution was concentrated and purified using flash column chromatography over silica gel $\left(H / \mathrm{Et}_{2} \mathrm{O}=1: 9\right)$. The title compound was isolated as a colourless oil (16 mg, $0.075 \mathrm{mmol}, 55 \%, \mathbf{9 5 \%} \boldsymbol{e e}$ ).

${ }^{1}$ H NMR $\left(400 \mathrm{MHz}, \mathrm{CDCl}_{3}\right) \delta 7.14(\mathrm{~s}, 1 \mathrm{H}), 7.04(\mathrm{~s}, 1 \mathrm{H}), 6.69(\mathrm{~d}, J=2.2 \mathrm{~Hz}, 1 \mathrm{H}), 4.01(\mathrm{~s}, 1 \mathrm{H})$, $3.53(\mathrm{~m}, 1 \mathrm{H}), 3.45(\mathrm{dd}, J=16.9,5.1 \mathrm{~Hz}, 1 \mathrm{H}), 3.29(\mathrm{dd}, J=16.9,9.0 \mathrm{~Hz}, 1 \mathrm{H}), 2.64-2.46(\mathrm{~m}, 2 \mathrm{H})$, 2.36-2.27 (m, 2H), $1.67(d d t, J=13.0,8.9,6.3 \mathrm{~Hz}, 1 \mathrm{H})$.

${ }^{13} \mathrm{C}$ NMR $\left(101 \mathrm{MHz}, \mathrm{CDCl}_{3}\right) \delta 190.5,149.4,129.3,127.2,118.4,116.2,43.1,42.6,36.2,32.7$, 29.8 .

HRMS (ESI) m/z: $[\mathrm{M}+\mathrm{H}]^{+}$calcd for $\mathrm{C}_{12} \mathrm{H}_{14} \mathrm{~N}_{3} \mathrm{O}$ 216.1137; found: 216.1123 .

IR $\left(\mathrm{cm}^{-1}\right):$ 2926, 2855, 2220, 1676, 1467, 1411.

$[\boldsymbol{a}]_{D}^{20}=-98.4\left(c 0.33, \mathrm{CH}_{2} \mathrm{Cl}_{2}\right)$

HPLC analysis: carried out using a mixture of hexane and isopropyl alcohol (85:15 isocratic), at $1 \mathrm{~mL} \cdot \mathrm{min}^{-1}$, at $30{ }^{\circ} \mathrm{C}$ on an IC column, with UV detection $(\lambda=280 \mathrm{~nm})$. Retention times: 30.11 and $32.56 \mathrm{~min}$. 


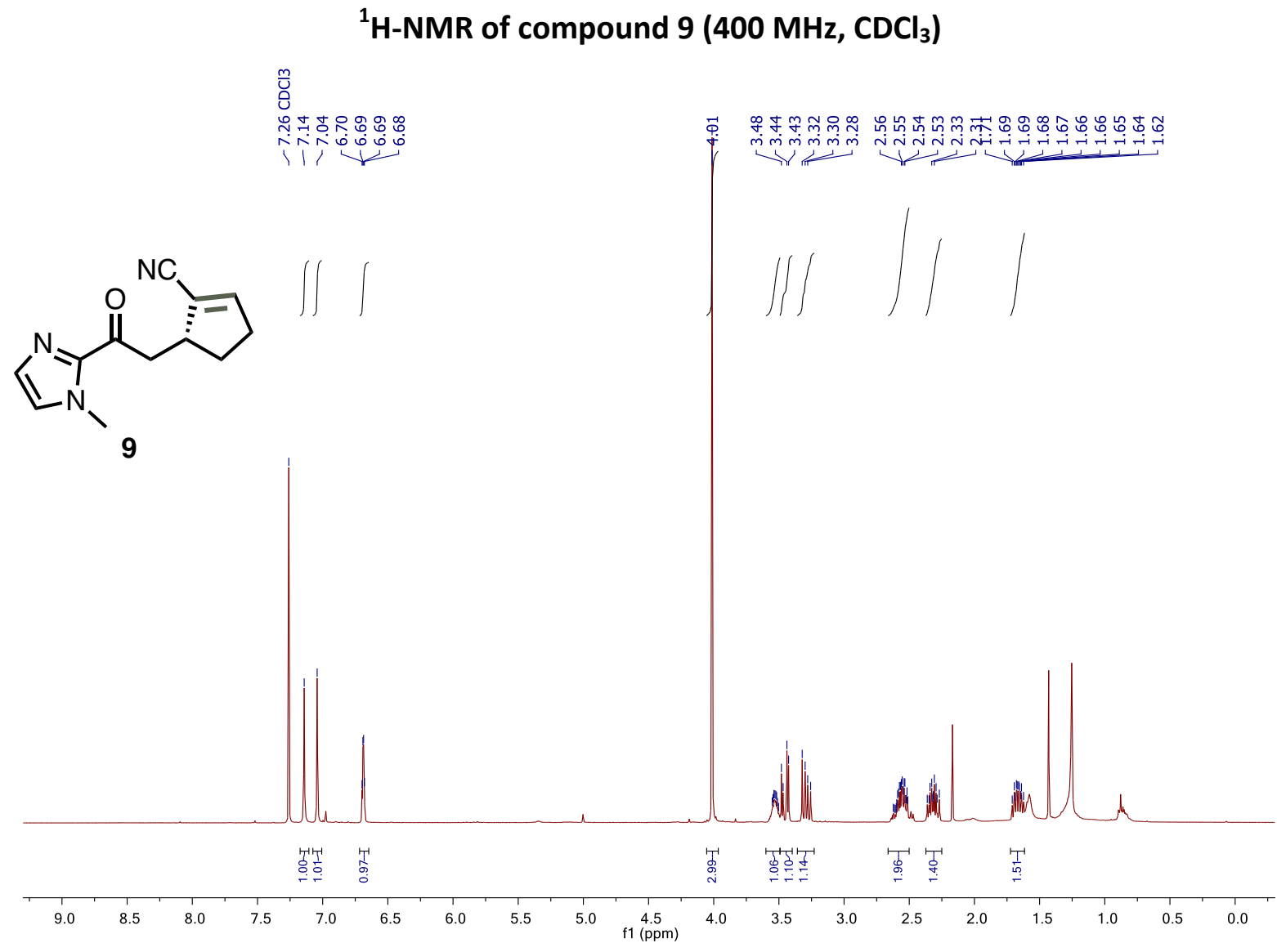

${ }^{13} \mathrm{C}-\mathrm{NMR}$ of compound $9\left(101 \mathrm{MHz}, \mathrm{CDCl}_{3}\right)$

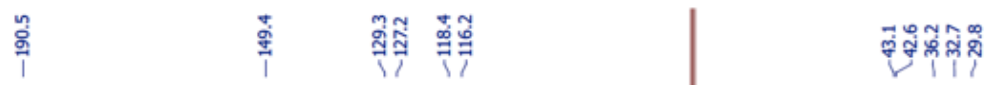



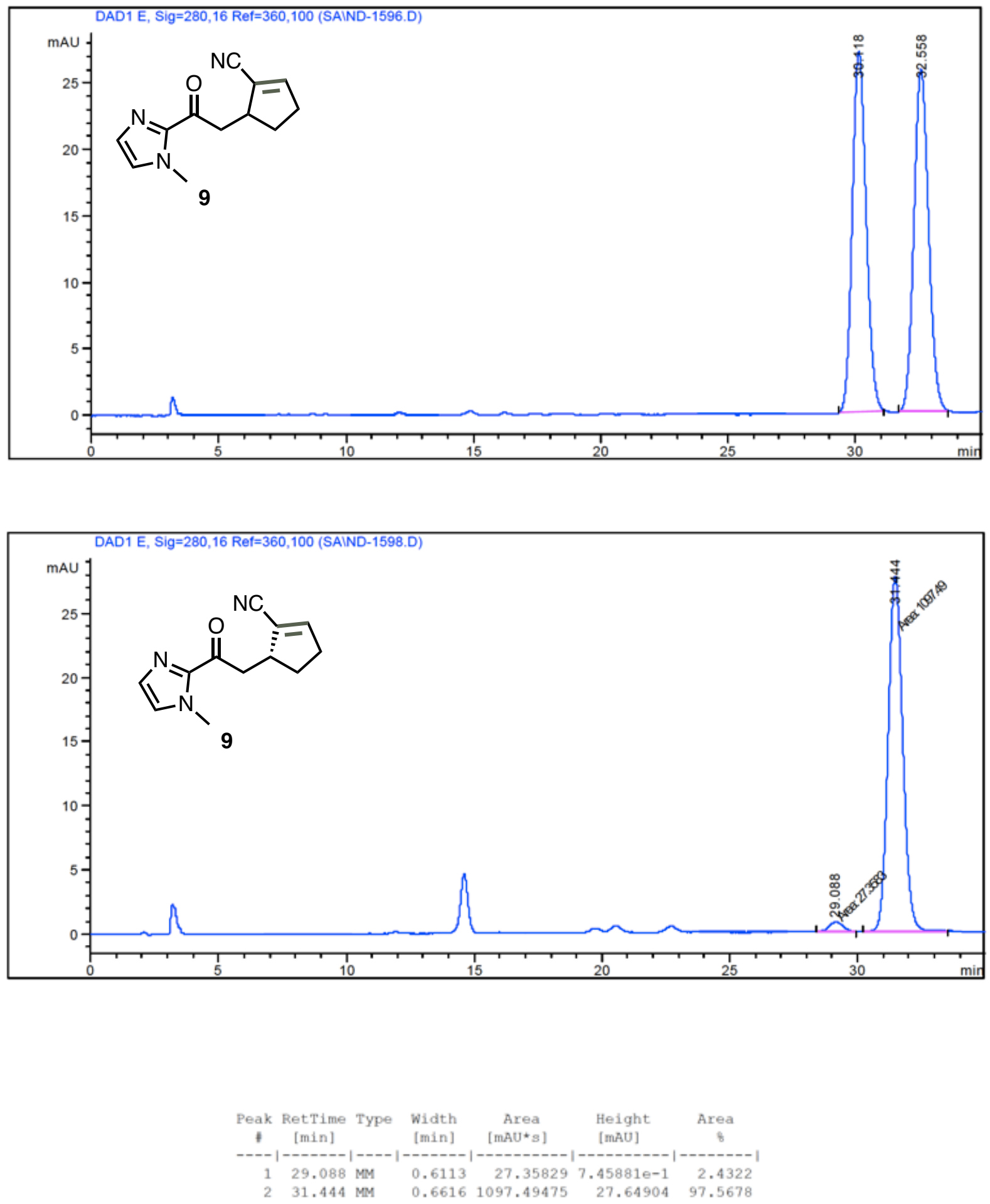
Diethyl 2-methylene-3-phenylpentanedioate (10)

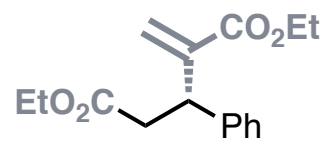

Molecular formula: $\mathrm{C}_{16} \mathrm{H}_{20} \mathrm{O}_{4}$

$\mathbf{M W}=276.33 \mathrm{~g} \cdot \mathrm{mol}^{-1}$

To a solution of 3-phenyl-5-(1-methyl-1H-imidazol-2-yl)-2-methylene-5-oxopentanenitrile 3m (110 mg, 0.414 mmol, 1 equiv.) in dry DCM (4 mL) was added methyl trifluoromethanesulfonate $\left(124 \mu \mathrm{L}, 0.830 \mathrm{mmol}, 2.0\right.$ equiv.) at $0{ }^{\circ} \mathrm{C}$ under argon. Upon completion $(1 \mathrm{~h})$, ethanol $(500 \mu \mathrm{L})$ and DBU $(500 \mu \mathrm{L})$ were slowly added successively. The resulting mixture was stirred at RT for 30 min, quenched with a saturated aqueous solution of $\mathrm{NaHCO}_{3}(4 \mathrm{~mL})$ and the aqueous phase was extracted with DCM $(2 \times 20 \mathrm{~mL})$. The combined organic layers were concentrated and dissolved in ethanol $(1 \mathrm{~mL})$. The resulting solution was cooled down to $0{ }^{\circ} \mathrm{C}$ and $\mathrm{H}_{2} \mathrm{SO}_{4}$ (500 $\mu \mathrm{L}$ ) was slowly added and the reaction mixture was heated at $80^{\circ} \mathrm{C}$ for $5 \mathrm{~h}$ using a heating mantle. Upon completion, the solution was allowed to cooled down to $0{ }^{\circ} \mathrm{C}$ and quenched with a saturated aqueous solution of $\mathrm{NaHCO}_{3}(4 \mathrm{~mL})$. The aqueous phase was extracted with DCM $(3 \times 10 \mathrm{~mL})$ and the resulting crude was purified using flash column chromatography over silica gel (H/EtOAc $=9: 1)$. The title compound was isolated as a colourless oil ( $46 \mathrm{mg}, 0.166 \mathrm{mmol}, 40 \%, 79 \%$ ee).

${ }^{1}$ H NMR $\left(400 \mathrm{MHz}, \mathrm{CDCl}_{3}\right) \delta$ 7.29-7.17 (m, 5H), $6.32(\mathrm{~s}, 1 \mathrm{H}), 5.64(\mathrm{~d}, J=0.7 \mathrm{~Hz}, 1 \mathrm{H}), 4.44$ (t, J=7.9 Hz, 1H), 4.16-4.03 (m, 4H), 2.91 (dd, $J=15.5,7.9 \mathrm{~Hz}, 1 \mathrm{H}), 2.78$ (dd, $J=15.5,8.1 \mathrm{~Hz}$, $1 \mathrm{H}), 1.20(\mathrm{t}, J=7.1 \mathrm{~Hz}, 3 \mathrm{H}), 1.15(\mathrm{t}, J=7.1 \mathrm{~Hz}, 3 \mathrm{H})$.

${ }^{13} \mathrm{C}$ NMR $\left(101 \mathrm{MHz} \mathrm{CDCl}_{3}\right) \delta 171.5,166.3,142.7,141.3,128.4,127.9,126.8,124.2,60.8$, $60.5,42.8,39.7,14.1,14.0$.

HRMS (ESI) m/z: [M+H] calcdd for $\mathrm{C}_{16} \mathrm{H}_{21} \mathrm{O}_{4}$ 277.1440; found: 277.1431 .

IR $\left(\mathrm{cm}^{-1}\right): 2983,2933,1736,1721,1631,1372,1248,1141,1032$.

$[\boldsymbol{a}]_{\boldsymbol{D}}^{\mathbf{2 0}}=-56.2\left(c 0.63, \mathrm{CH}_{2} \mathrm{Cl}_{2}\right)$

HPLC analysis: carried out using a mixture of hexane and isopropyl alcohol (90:10 isocratic), at $1 \mathrm{~mL} \cdot \mathrm{min}^{-1}$, at $30{ }^{\circ} \mathrm{C}$ on an IC column, with UV detection $(\lambda=230 \mathrm{~nm})$. Retention times: 8.50 and $15.08 \mathrm{~min}$. 
${ }^{1} \mathrm{H}-\mathrm{NMR}$ of compound $10\left(400 \mathrm{MHz}, \mathrm{CDCl}_{3}\right)$

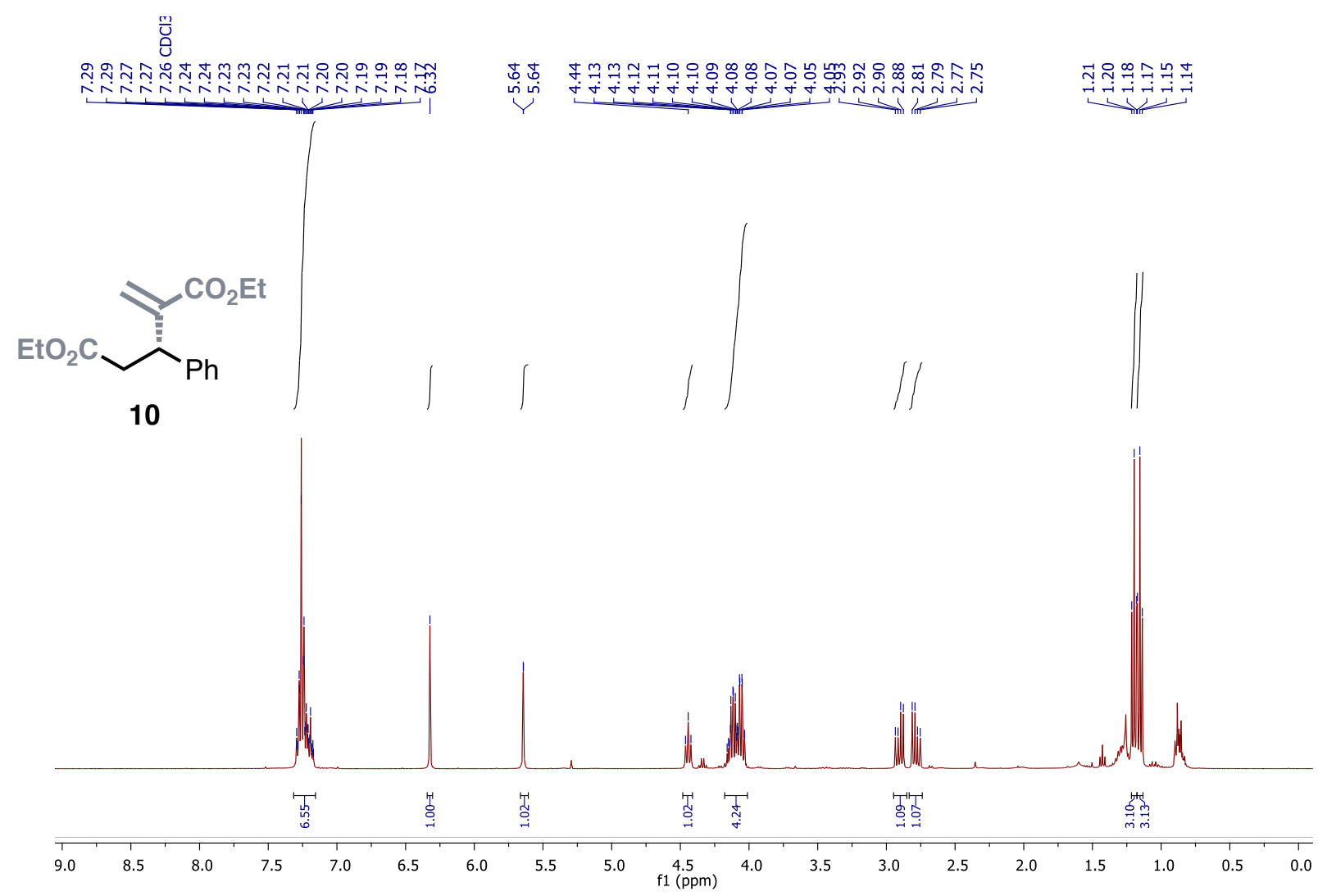

${ }^{13} \mathrm{C}-\mathrm{NMR}$ of compound $10\left(101 \mathrm{MHz}, \mathrm{CDCl}_{3}\right)$

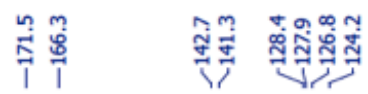

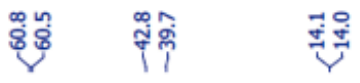

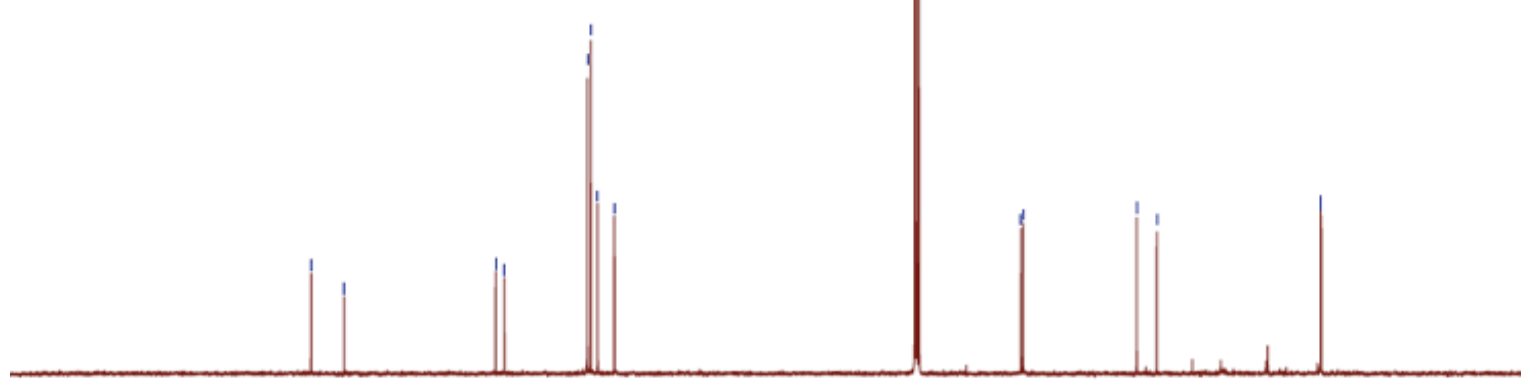

$\begin{array}{rllllllllllllllllllllllll} & 210 & 200 & 190 & 180 & 170 & 160 & 150 & 140 & 130 & 120 & 110 & 100 & 90 & 80 & 70 & 60 & 50 & 40 & 30 & 20 & 10 & 0 & -10\end{array}$ 

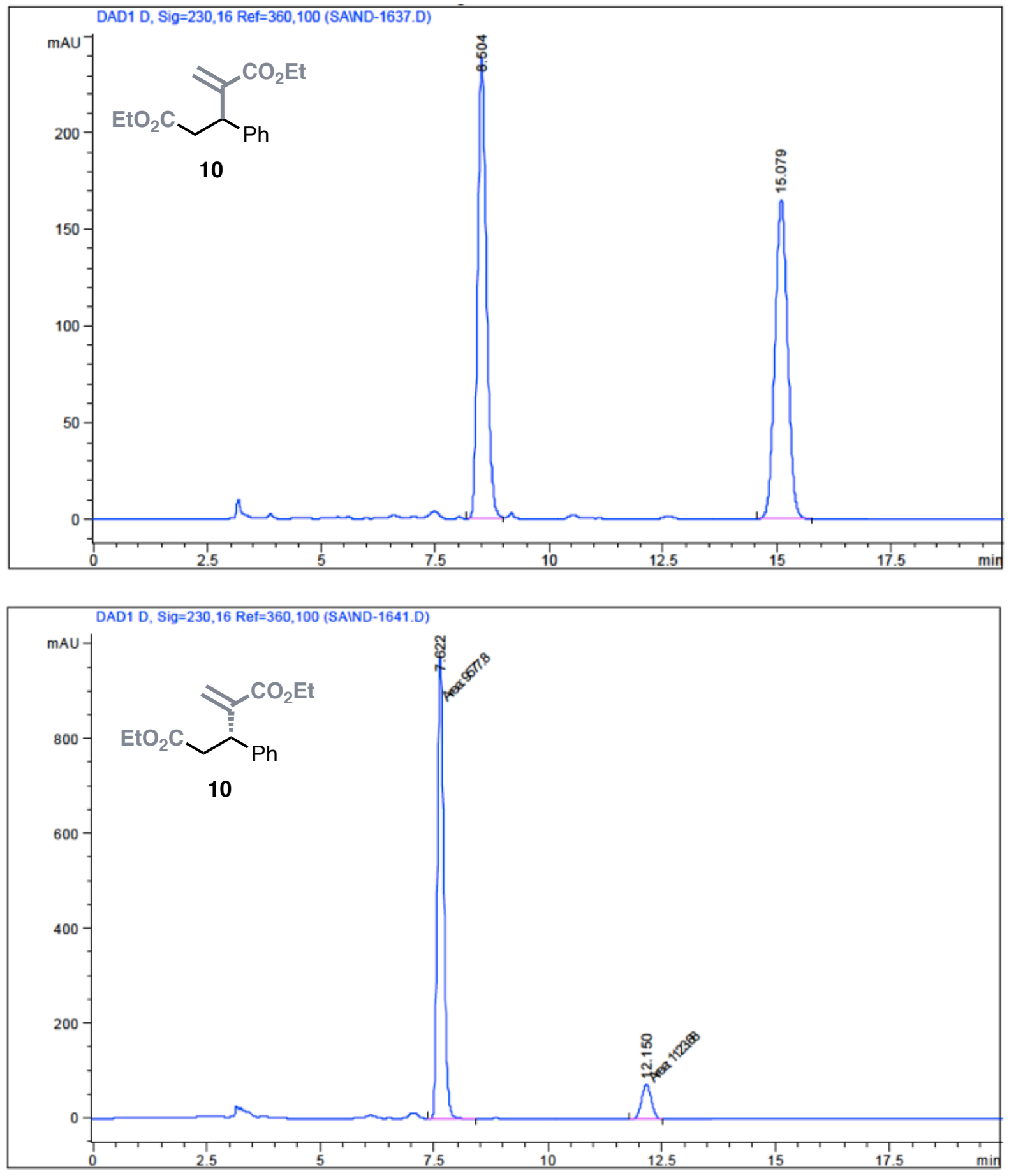

\begin{tabular}{|c|c|c|c|c|c|c|}
\hline $\begin{array}{c}\text { Peak } \\
\#\end{array}$ & $\begin{array}{c}\text { RetTime } \\
\text { [min] }\end{array}$ & Type & $\begin{array}{l}\text { Width } \\
\text { [min] }\end{array}$ & $\begin{array}{c}\text { Area } \\
{\left[\mathrm{mAU}{ }^{*} \mathrm{~s}\right]}\end{array}$ & $\begin{array}{l}\text { Height } \\
\text { [mAU] }\end{array}$ & $\begin{array}{c}\text { Area } \\
\text { \&ิ }\end{array}$ \\
\hline - & 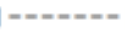 & & (--n-s. & 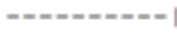 & | & | \\
\hline 1 & 7.622 & VM & 0.1635 & 9577.79688 & 976.56189 & 89.4998 \\
\hline 2 & 12.150 & MM & 0.2506 & 1123.67810 & 74.73367 & 10.5002 \\
\hline
\end{tabular}




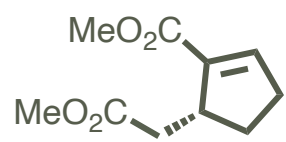

Molecular formula: $\mathrm{C}_{10} \mathrm{H}_{14} \mathrm{O}_{4}$

$\mathrm{MW}=198.22 \mathrm{~g} \cdot \mathrm{mol}^{-1}$

To a solution of 5-[2-(1-Methyl-1H-imidazol-2-yl)-2-oxoethyl]cyclopent-1-ene-1-carbonitrile 9 (12 mg, $0.055 \mathrm{mmol})$ in dry DCM $(0.5 \mathrm{~mL})$ was added methyl trifluoromethanesulfonate (41 $\mu \mathrm{L}, 0.275 \mathrm{mmol}, 5.0$ equiv.) at $0{ }^{\circ} \mathrm{C}$ under argon. Upon completion ( $2 \mathrm{~h}$ ), $\mathrm{MeOH}(400 \mu \mathrm{L})$ and DBU $(200 \mu \mathrm{L})$ were slowly added successively. The resulting mixture was stirred at RT for $4 \mathrm{~min}$, quenched with a saturated aqueous solution of $\mathrm{NaHCO}_{3}(2 \mathrm{~mL})$ and the aqueous phase was extracted with DCM $(3 \times 10 \mathrm{~mL})$. The combined organic layers were concentrated and dissolved in $\mathrm{MeOH}(1 \mathrm{~mL})$. The resulting solution was cooled down to $0{ }^{\circ} \mathrm{C}$ and $\mathrm{H}_{2} \mathrm{SO}_{4}$ $(500 \mu \mathrm{L})$ was slowly added and the reaction mixture was heated at $80{ }^{\circ} \mathrm{C}$ for $5 \mathrm{~h}$ using a heating mantle. Upon completion, the solution was allowed to cooled down to $0{ }^{\circ} \mathrm{C}$ and quenched with a saturated aqueous solution of $\mathrm{NaHCO}_{3}(4 \mathrm{~mL})$. The aqueous phase was extracted with DCM $(3 \times 10 \mathrm{~mL})$ and the resulting crude residue was purified by flash column chromatography over silica gel $\left(\mathrm{Hex} / \mathrm{Et}_{2} \mathrm{O}=4: 1\right)$. The title compound was isolated as a colourless oil $(6.2 \mathrm{mg}, 0.03 \mathrm{mmol}, 57 \%, 95 \%$ ee). Spectral data matched those reported in the literature. ${ }^{19}$

${ }^{1} \mathrm{H}$ NMR $\left(400 \mathrm{MHz}, \mathrm{CDCl}_{3}\right) \delta 6.83(\mathrm{dd}, J=4.3,2.6 \mathrm{~Hz}, 1 \mathrm{H}), 3.73(\mathrm{~s}, 3 \mathrm{H}), 3.68(\mathrm{~s}, 3 \mathrm{H}), 3.35(\mathrm{~m}$, 1H), $2.86(\mathrm{dd}, J=15.4,3.9 \mathrm{~Hz}, 1 \mathrm{H}), 2.53-2.43(\mathrm{~m}, 2 \mathrm{H}), 2.28(\mathrm{~m}, 1 \mathrm{H}), 1.72$ (ddd, $J=18.0,8.9$, 4.7 Hz, 1H).

${ }^{13} \mathrm{C}$ NMR $\left(101 \mathrm{MHz}, \mathrm{CDCl}_{3}\right) \delta 173.1,165.2,145.3,138.0,51.5,51.4,40.9,38.3,31.4,29.9$. $[\boldsymbol{a}]_{\boldsymbol{D}}^{\mathbf{2 4}}=+23.9\left(\mathrm{c} 0.48, \mathrm{CHCl}_{3}\right)\left\{[\boldsymbol{a}]_{\boldsymbol{D}}^{26}+30.7\left(\mathrm{c} 0.6, \mathrm{CHCl}_{3}\right)\left(\mathrm{lit}^{19}\right)\right\}$.

HPLC analysis: carried out using a mixture of hexane and isopropyl alcohol (90:10 isocratic), at $1 \mathrm{~mL} \cdot \mathrm{min}^{-1}$, at $30{ }^{\circ} \mathrm{C}$ on an IC column, with UV detection $(\lambda=230 \mathrm{~nm})$. Retention times: 10.54 and $13.81 \mathrm{~min}$.

\footnotetext{
${ }^{19}$ Urones, J. G.; Garrido, D. D.; Diez, D.; El Hammoumi, M. M.; Dominguez, S. H.; Casaseca, J. A.; Davies, S. G.; Smith, A. D. Org. Biomol. Chem. 2004, 2, 364-372.
} 
${ }^{1} \mathrm{H}-\mathrm{NMR}$ of compound $11\left(400 \mathrm{MHz}, \mathrm{CDCl}_{3}\right)$

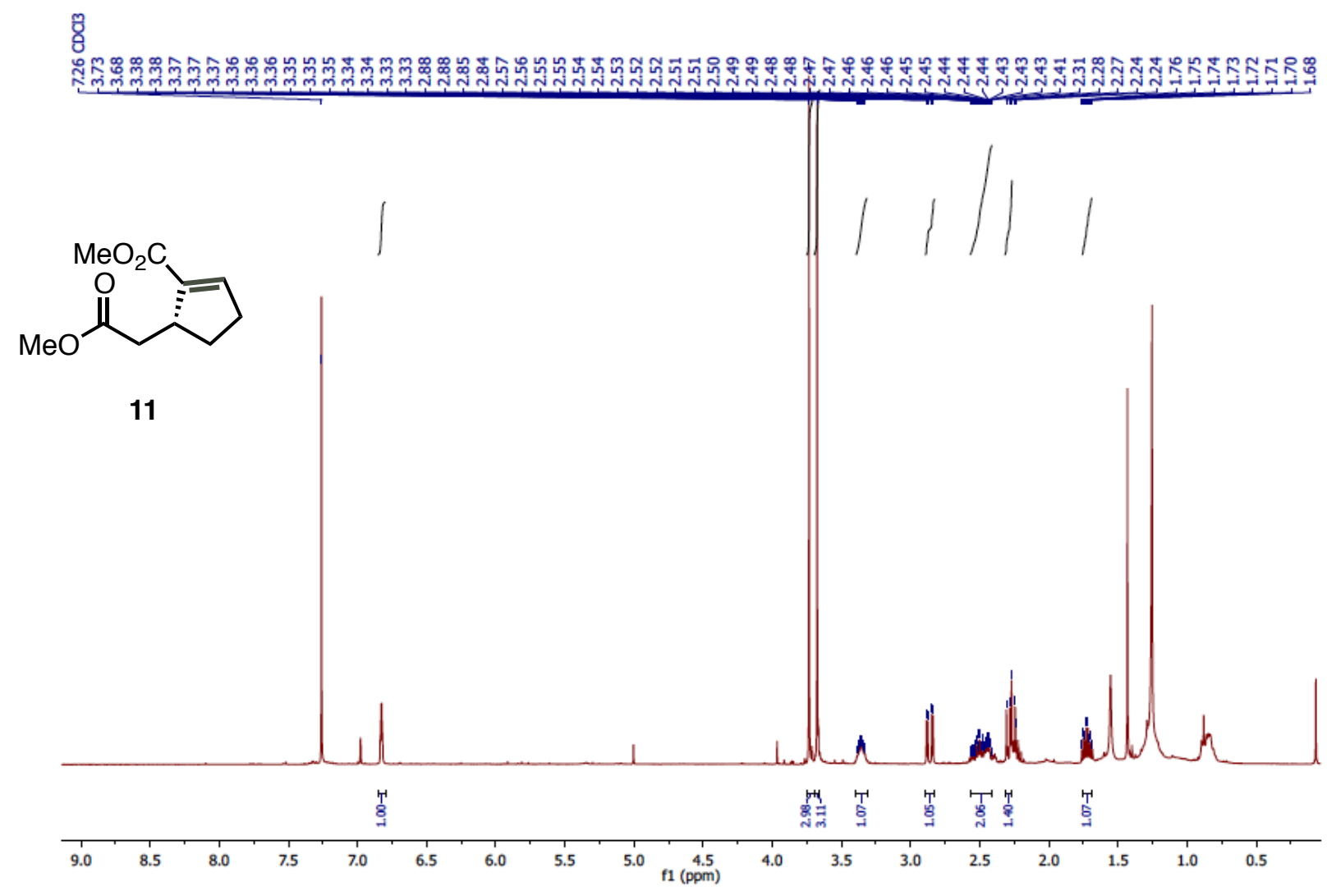

${ }^{13} \mathrm{C}-\mathrm{NMR}$ of compound $11\left(101 \mathrm{MHz}, \mathrm{CDCl}_{3}\right)$

$$
\text { 具 }
$$

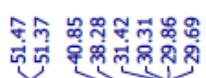

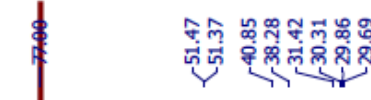

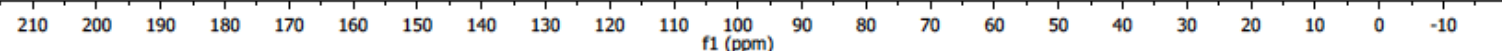



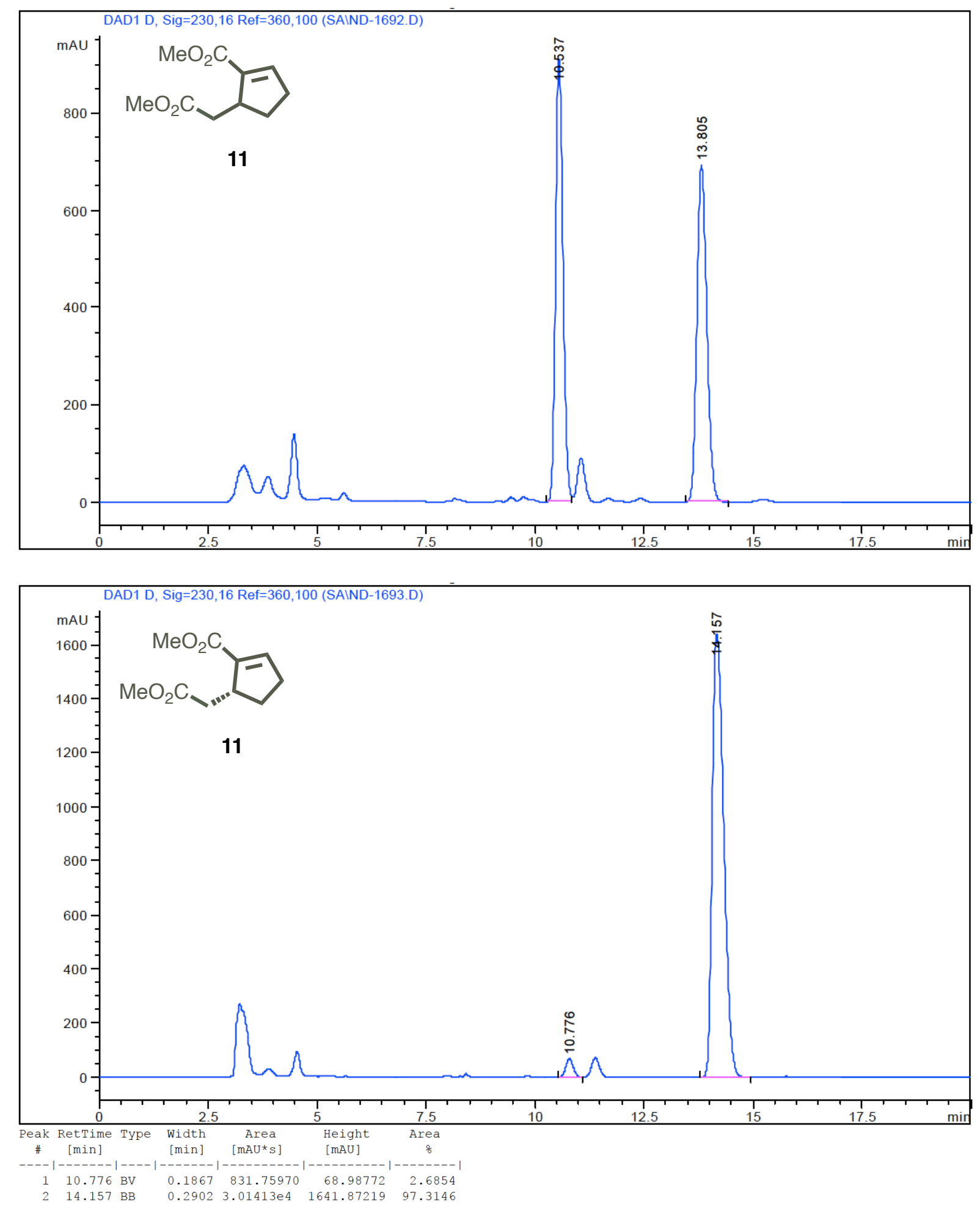
General procedure $\mathbf{G}$ for the asymmetric Cu-Catalyzed sequence
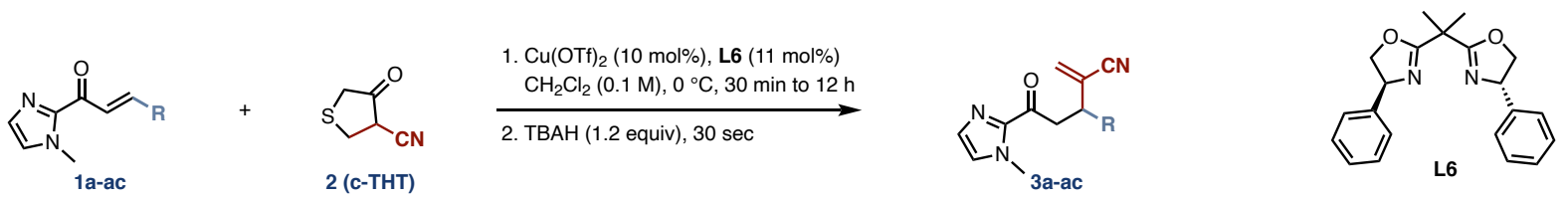

An oven-dried reaction vial was charged with $\mathrm{Cu}(\mathrm{OTf})_{2}$ (Alfa Aesar) (10 mg, $0.027 \mathrm{mmol}$, 0.1 equiv.) and (+)-2,2'-Isopropylidene-bis[(4R)-4-phenyl-2-oxazoline] L6 (Sigma-Aldrich) (10 mg, $0.03 \mathrm{mmol}, 0.11$ equiv.) then dried for $1 \mathrm{~h}$ under high-vacuum. Dry DCM (2 mL) was added, and catalyst formation was ensured by stirring the resulting solution for $1 \mathrm{~h}$ at $\mathrm{rt}$. Enone $\mathbf{x}$-xliii ( $0.27 \mathrm{mmol}, 1.0$ equiv.) was also dried under high-vacuum for $15 \mathrm{~min}$, dissolved in dry DCM $(0.7 \mathrm{~mL})$. The resulting solution was cooled to $0{ }^{\circ} \mathrm{C}$, and c-THT (4-cyano-3oxotetrahydrothiophene) (38 mg, $0.3 \mathrm{mmol}, 1.1$ equiv.) was added. Upon completion of the reaction as indicated by TLC (Toluene/Acetone $=9: 1$ ), or if the reaction was not complete, after $2 \mathrm{~d}$, TBAH (1.2 equiv.) was added and the reaction mixture was stirred for $2 \mathrm{~min}$. The reaction mixture was then diluted with a saturated aqueous solution of $\mathrm{NaHCO}_{3}(5 \mathrm{~mL})$ and the aqueous phase was extracted with DCM $(3 \times 10 \mathrm{~mL})$. The combined organic layers were dried over anhydrous $\mathrm{MgSO}_{4}$ and concentrated. The crude residue was purified by flash column chromatography over silica gel using either $\mathrm{Hex} / \mathrm{Et}_{2} \mathrm{O}$, Hex/EtOAc or Hex/EtOAc/Acetone eluant mixtures.

\section{Cu-catalyzed sequence: Scope}
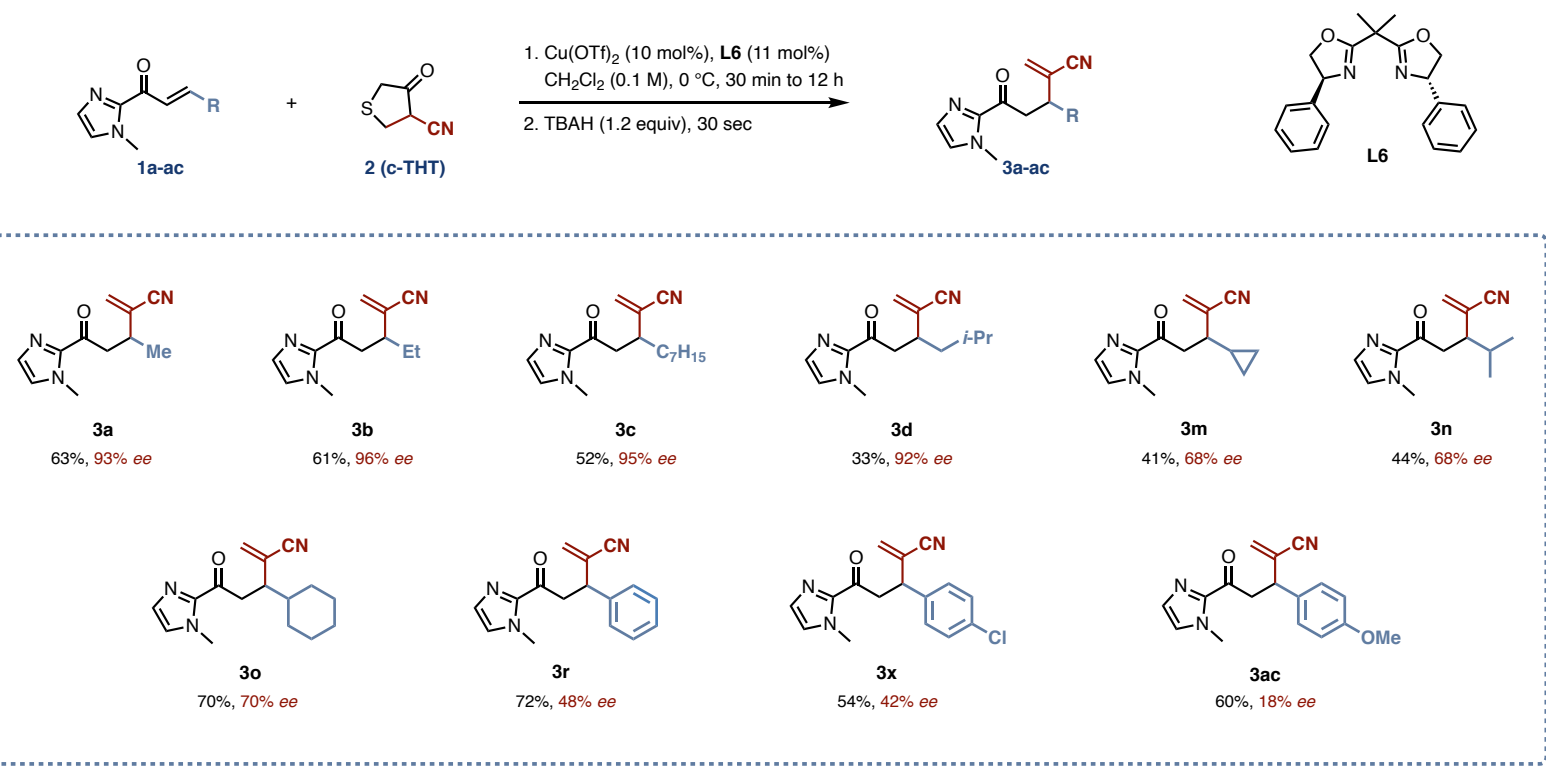